\title{
Nonlinear and Nonequilibrium Dynamics of Quantum-Dot Optoelectronic Devices
}

\author{
vorgelegt von \\ MSc. Benjamin Clemens Oliver Lingnau \\ aus Berlin
}

Von der Fakultät II - Mathematik und Naturwissenschaften

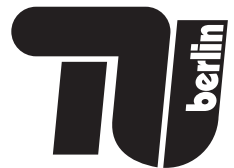

der Technischen Universität Berlin

zur Erlangung des akademischen Grades

Doktor der Naturwissenschaften

- Dr. rer. nat. -

genehmigte Dissertation

Promotionsausschuss:

Vorsitzender: $\quad$ Prof. Dr. Michael Lehmann

Berichter: $\quad$ Prof. Dr. Eckehard Schöll, PhD

Berichterin: Prof. Dr. Kathy Lüdge

Berichter: $\quad$ Prof. Dr. Weng W. Chow

Tag der wissenschaftlichen Aussprache: 13. April 2015

Berlin 2015

D 83 



\section{Abstract}

In this thesis the dynamics and performance of optoelectronic devices based on semiconductor quantum-dots are investigated.

In the first part, the dynamics of quantum-dot lasers under external perturbations is discussed. Using a microscopically based balance equation model that incorporates detailed charge-carrier scattering dynamics and the possibility to describe nonequilibrium between intra-band electronic states, the relaxation oscillations of the quantum-dot laser are investigated. Three qualitatively different dynamic regimes are identified in dependence of the scattering rates - the "constantreservoir" regime for slow scattering, the "overdamped" regime, and the "synchronized" regime for high scattering - characterized by a varying degree of nonequilibrium between the quantum-dot and reservoir states.

Important differences to conventional lasers are found in the modulation response and the dynamics in optical injection and feedback setups. Common theoretical models and approaches used to describe these applications are shown to yield inaccurate predictions, especially in the "constant-reservoir" and "overdamped" dynamic regimes. An important consequence is that the amplitude-phase coupling in quantum-dot lasers, commonly described by the $\alpha$-factor, differs from conventional descriptions due to the desynchronization of gain and refractive index. While the $\alpha$-factor describes bifurcations of fixed points accurately, it fails in describing dynamic solutions and overestimates the extent of complex dynamics. The observed low sensitivity to optical perturbations in quantum-dot lasers can therefore be attributed partly to the charge-carrier nonequilibrium. Three quantum-dot laser models on different levels of sophistication are presented that can accurately describe the quantum-dot nonequilibrium dynamics.

In the second part of the thesis, the performance of quantum-dot semiconductor optical amplifiers is investigated, and two types of applications unique to quantum-dots as active medium are discussed. The ground and excited states of the quantum-dots allow an ultra-broad-band amplification of optical data streams. Amplified signals on the ground-state frequencies are shown to generally exhibit higher quality than on the excited state, due to a lower sensitivity of the groundstate to carrier-density variations. Nevertheless the quantum-dot amplifier is found to allow effective amplification on both frequency ranges. Furthermore, a parameter range is identified that allows for a simultaneous amplification of data signals on the ground and excited state in a counter-propagating setup.

The long microscopically polarization dephasing times in quantum-dots are found to enable quantum-coherent interactions on a macroscopic scale at room-temperature. By comparison with experiments, the occurrence of Rabi-oscillations by amplification of ultra-short pulses is demonstrated. Quantum-dot based devices could therefore be used for future applications based on quantum-coherent effects. 


\section{Zusammenfassung}

In dieser Dissertation werden die Dynamik und die Eigenschaften von optoelektronischen Bauteilen auf Basis von Halbleiter-Quantenpunkten untersucht.

Im ersten Teil wird die Dynamik von Quantenpunktlasern unter dem Einfluss externer Störungen diskutiert. Mithilfe eines mikroskopisch basierten Bilanzgleichungsmodells, das die detaillierten Ladungsträger-Streuprozesse und Nichtgleichgewichtszustände beschreiben kann, werden die Relaxationsoszillationen von Quantenpunktlasern untersucht. In Abhängigkeit von den Streuraten werden drei qualitativ unterschiedliche dynamische Bereiche identifiziert - der "constant-reservoir", der "overdamped", sowie der "synchronized" Bereich - die durch einen unterschiedlichen Grad des Nichtgleichgewichts zwischen Quantenpunkt- und Reservoirzuständen charakterisiert sind.

Im Vergleich zu konventionellen Lasern treten wichtige Unterschiede bezüglich des Verhaltens unter Pumpstrommodulation und unter optischer Injektion oder Rückkopplung auf. Theoretische Modelle, die typischerweise zur Beschreibung dieser Anwendungen verwendet werden, liefern nur ungenaue Ergebnisse. Eine wichtige Konsequenz ist, dass die Amplituden-Phasen-Kopplung in Quantenpunktlasern, die häufig durch den $\alpha$-Faktor beschrieben wird, von diesem aufgrund der Desynchronisation von optischem Gewinn und Brechungsindexänderung abweicht. Dadurch werden dynamische Lösungen, insbesondere unter optischer Störung des Lasers, von etablierten Modellen nur ungenau beschrieben, und deren Ausdehnung überschätzt. Die geringe Empfindlichkeit von Quantenpunktlasern gegnüber optischen Störungen lässt sich daher teilweise mit dem Ladungsträgernichtgleichgewicht erklären. Drei Quantenpunktlasermodelle unterschiedlicher Komplexität werden vorgestellt, die die Nichtgleichgewichtsdynamik korrekt darstellen.

Im zweiten Teil der Dissertation wird die Leistungsfähgkeit von Halbleiter-Quantenpunkt-basierten optischen Verstärkern in zwei unterschiedlichen Anwendungen untersucht. Die Grund- und angeregten Zustände der Quantenpunkte ermöglichen eine optische Verstärkung von optischen Datenströmen mit ultra-hoher optischer Bandbreite. Aufgrund der geringen Empfindlichkeit des Grundzustands bezüglich Änderungen der Ladungsträgerdichte können Signale auf den Grundzustandsfrequenzen generell mit höherer Signalqualität verstärkt werden können als auf dem angereten Zustand. Dennoch können Quantenpunktverstärker Signale in beiden Wellenlängenbereichen effektiv verstärken. Außerdem wird ein Parameterbereich identifiziert, der eine gleichzeitige Verstärkung von zwei Signalen im Frequenzbereich der beiden Quantenpunktzustände ermöglicht.

Die lange Lebensdauer der mikroskopischen Polarisation in Quantenpunkten ermöglicht quantenkohärente Wechselwirkungen auf einer makroskopischen Skala bei Zimmertemperatur. Anhand eines Vergleichs mit experimentellen Ergebnissen wird das Auftreten von Rabioszillationen bei der Verstärkung von ultrakurzen optischen Pulsen gezeigt. Quantenpunktbauteile könnten daher in Zukunft Anwendungen ermöglichen, die auf quantenkohärenten Effekten beruhen. 
1. Introduction 1

1.1. Semiconductor Lasers . . . . . . . . . . . . . . . . . . 2

1.2. Semiconductor Lasers as Dynamical Systems . . . . . . . . . . . 3

1.3. Semiconductor Quantum-Dots . . . . . . . . . . . . . . . . . 4

1.4. Outline of the Thesis . . . . . . . . . . . . . . 7

2. Theory of Quantum-Dot Optical Devices 9

2.1. Introduction . . . . . . . . . . . . . . . . . 9

2.2. Charge-Carrier Scattering in Quantum-Dot Structures . . . . . . . 11

2.2.1. Coulomb-Scattering of Charge Carriers . . . . . . . . . 13

2.2.2. Electron-Hole Picture . . . . . . . . . . . . . . 17

2.2.3. Detailed Balance . . . . . . . . . . . . . . . . . 18

2.2.4. Carrier-Phonon Scattering . . . . . . . . . . . . . 20

2.3. Light-Matter Interaction . . . . . . . . . . . . . . . . 23

2.3.1. Electric Field Dynamics . . . . . . . . . . . . . . 23

2.3.2. Maxwell-Bloch Equations . . . . . . . . . . . . 27

2.4. Quantum-Dot Laser Rate Equations . . . . . . . . . . . . . . . . 31

2.4.1. Maxwell-Bloch Laser Rate Equations . . . . . . . . . . . . . 31

2.4.2. Adiabatically Eliminated Polarization . . . . . . . . . . 36

2.4.3. Modeling of Spontaneous Emission . . . . . . . . . . . . 38

2.4.4. Carrier-Induced Gain and Refractive Index Changes . . . . . 39

2.5. Quantum-Dot Laser Carrier-Heating Model . . . . . . . . . . . . . . 42

2.5.1. Charge-Carrier Energy and Temperature . . . . . . . . . 42

2.5.2. Carrier Heating by Auger-Scattering Processes . . . . . . . . 44

2.5.3. Energy Balance Equations . . . . . . . . . . . . . . 45 
3. Quantum-Dot Laser Dynamics 47

3.1. Introduction . . . . . . . . . . . . . . . . . . . 47

3.2. Laser Dynamics - Relaxation Oscillations . . . . . . . . . . . . . 49

3.2.1. Relaxation Oscillations in Two-Variable Laser Equations . . . 49

3.2.2. Turn-On Dynamics of Quantum-Dot Lasers . . . . . . . . . . 53

3.2.3. Influence of Charge-Carrier Scattering . . . . . . . . . . 57

3.3. Minimal Model for Quantum-Dot Laser Dynamics . . . . . . . . . . 62

3.3.1. Linearization and Eigenvalue Problem . . . . . . . . . . . . 64

3.3.2. Asymptotic Analysis - Relaxation Oscillations . . . . . . . . 67

3.4. Modulation Response of Quantum-Dot Lasers . . . . . . . . . . . . 72

3.4.1. Small-Signal Response . . . . . . . . . . . . . . . . . . 72

3.5. Amplitude-Phase Coupling in Quantum-Dot Lasers . . . . . . . . . . 79

3.5.1. The Linewidth-Enhancement Factor $\alpha \ldots \ldots$. . . . . . 80

3.5.2. Charge-Carrier-Induced Susceptibility in Quantum-Dot Lasers 82

3.6. Dynamics under Optical Injection . . . . . . . . . . . . . . . . . 88

3.6.1. Quantum-Dot Laser Model with Optical Injection . . . . . . 89

3.6.2. Injection Locking of Quantum-Dot Lasers . . . . . . . . . . 91

3.6.3. Dependence on the Quantum-Dot Structure and Pump-Current 97

3.6.4. Evaluation of the $\alpha$-Factor from Optical Injection . . . . . . 100

3.6.5. Comparison with $\alpha$-Factor-Based Models . . . . . . . . 103

3.7. Optical Injection - Numerical Path Continuation . . . . . . . . . . . 113

3.7.1. Quantum-Dot Laser Model Simplification . . . . . . . . . . . 113

3.7.2. Path Continuation Results . . . . . . . . . . . . . 119

3.7.3. Dependencies on Scattering and Reservoir Loss Rates . . . . 122

3.7.4. Summary . . . . . . . . . . . . . . . . . . . . . . 124

3.8. Dynamics under Optical Feedback . . . . . . . . . . . . . . 125

3.8.1. Quantum-Dot Laser Model with Optical Feedback . . . . . 126

3.8.2. Quantum-Dot Laser Dynamics under Optical Feedback . . . 127

3.9. Small-Signal Frequency Response of Quantum-Dot Lasers . . . . . . 134

3.9.1. Evaluation of the Frequency and Amplitude Modulation Indices 134

3.9.2. Numerical Evaluation of FM/AM Measurements . . . . . . . 135

3.9.3. Influence of Scattering Rates and Reservoir Losses . . . . . . 138

3.10. Conclusion . . . . . . . . . . . . . . . . . . . 141 
4.1. Introduction . . . . . . . . . . . . . . . . . . . . 145

4.2. Quantum-Dot Semiconductor Optical Amplifier Model . . . . . . . . 147

4.2.1. Electric Field Propagation . . . . . . . . . . . . . . 147

4.2.2. Quantum-Dot Material Equations . . . . . . . . . . . . 149

4.2.3. Modeling of Spontaneous Emission . . . . . . . . . . . . 150

4.3. Large-Signal Amplification in Quantum-Dot Amplifiers . . . . . . . . 154

4.3.1. Calculation of Amplified Spontaneous Emission Spectra . . . 154

4.3.2. Gain Saturation . . . . . . . . . . . . . . . 159

4.3.3. Amplification of Optical Data Streams . . . . . . . . . . . . . 161

4.4. Multi-State Operation of Quantum-Dot Amplifiers . . . . . . . . . 165

4.5. Coherent Transients in Quantum-Dot Amplifiers . . . . . . . . . . 171

4.5.1. Rabi-Oscillations in Quantum-Dot Semiconductor Amplifiers 172

4.5.2. Comparison with Experimental Measurements . . . . . . 177

4.6. Conclusion . . . . . . . . . . . . . . . . . . . . . 183

5. Summary and Outlook 185

$\begin{array}{lr}\text { A. Appendix } & 189\end{array}$

A.1. Evaluation of RO Parameters from Numerical Time-Series . . . . . . 189

A.2. Analytic Approximation of RO Parameters in the Synchronized Regime 189

$\begin{array}{ll}\text { List of Figures } & 191\end{array}$

$\begin{array}{ll}\text { List of Tables } & 197\end{array}$

$\begin{array}{lr}\text { Table of Symbols } & 199\end{array}$

$\begin{array}{ll}\text { List of Publications } & 201\end{array}$

Bibliography I

$\begin{array}{ll}\text { Acknowledgments } & \text { XXIX }\end{array}$ 


\section{INTRODUCTION}

Since their invention in 1960 [MAI60] lasers have been the topic of great physical interest and have been contributing to almost any technological field. Especially the importance of semiconductor lasers is undoubted, as they are the building blocks of many digital systems, telecommunication networks, and a variety of consumergrade products [BIM12, COL12]. Their small footprint and ease of integration, due to the possibility of electrical pumping, makes semiconductors the first choice for implementation of optoelectronic devices. Apart from lasers, semiconductor optoelectronic devices include optical amplifiers [ADA85a, OMA88, SCH88j, OLS89], electro-optic and electro-absorption modulators [KAM66, ISL87, ZUC88], and light-emitting diodes.

Semiconductor quantum-dots (QDs) are the final step in miniaturization of the semiconductor optically active material. Starting from three-dimensional bulk semiconductors, a reduction of the semiconductor material in one spatial dimension below the the de-Broglie wavelength of electrons (a few $\mathrm{nm}$ ) forms quantumwells (QWs) [DIN74]. These are quasi-two-dimensional semiconductor structures, in which the electron motion is confined in one dimension, leading to localized energy states. This localization leads to a strong increase in efficiency and a reduction of the threshold current in lasers, such that modern LEDs and semiconductor lasers almost always consist of quantum-well structures [COL12]. A confinement of electronic states in an additional dimension leads to quasi-one-dimensional quantum wires or dashes [LEL07]. When all three dimensions are small enough that electrons are confined, the localization leads to the formation of quasi-zero-dimensional, atom-like states, with sharply defined energies. These structures are semiconduc-

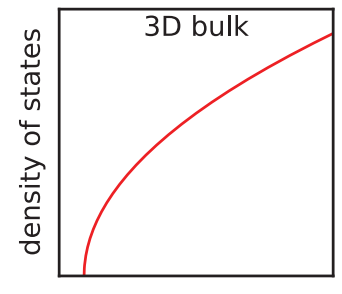

energy

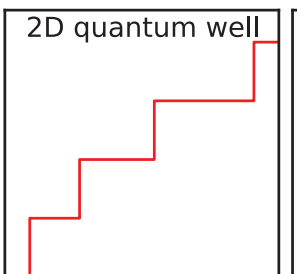

energy

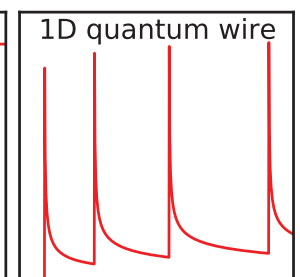

energy

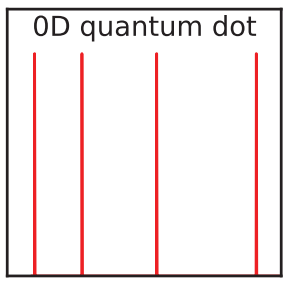

energy

Figure 1.1.: Sketch of the density of states depending on the dimensionality. 
tor quantum-dots [ARA82, BIM08a, CHO13a]. The dimensionality of the semiconductor structures determines their electronic density of states, as sketched in 1.1, which in turn determines their dynamical properties and device performance.

In the following, we will give a short introduction to semiconductor lasers and their dynamics, as well as a short overview on semiconductor quantum-dots.

\subsection{Semiconductor Lasers}

Semiconductor lasers in their simplest form consist of a single pn-junction within a laser cavity, formed by two mirrors. Due to the refractive-index difference between common semiconductor materials and air, nowadays the cleaved semiconductor facets provide high enough reflectivity to enable lasing (e.g. $R=0.31$ for the GaAs-air interface at $\lambda=1.3 \mu \mathrm{m}$ ). A prerequisite for the occurrence of stimulated emission of light is the inversion of the semiconductor medium, usually by electrical or optical pumping. In thermodynamic equilibrium the electron distribution of the semiconductor material would be described by a common Fermi-energy $E_{F}$, and the occupation probability by a Fermi-function:

$$
\rho(\varepsilon)=F\left(\varepsilon, E_{F}, T\right):=\left[1+\exp \left(\frac{\varepsilon-E_{F}}{k_{\mathrm{B}} T}\right)\right]^{-1},
$$

with the energy of the electronic state $\varepsilon$, the equilibrium temperature $T$ and Boltzmann's constant $k_{\mathrm{B}}$. An inversion of the electron distribution, i.e., a higher occupation of the energetically higher conduction band than in the valence band, is therefore inherently impossible under equilibrium conditions. The pump source thus drives the system towards nonequilibrium, injecting electrons into the conduction band and removing them from the valence band. This enables the stimulated emission of photons under recombination of a conduction-band electron with a vacant valence-band state (or "hole").

A recurring problem after the first demonstration of lasing in semiconductors was the confinement of charge-carriers. In order to restrict the injected electrons and holes to a well-defined region, a variety of modifications to the semiconductor structure has been made. The first, and most important, step was the proposal of using a heterostructure by Kroemer [KRO63]. The use of a semiconductor material (GaAs) in between a different material with higher band-gap (AlGaAs), sketched in Fig. $1.2 \mathrm{a}$, has then been successfully applied to provide room-temperature operation of semiconductor lasers in 1968 by Alferov [ALF68].

A further improvement was then achieved by development of the separate-confinement-heterostructure (SCH). Here, additional semiconductor layers with higher refractive index than the outer cladding layers were added on either side of the 
(a)

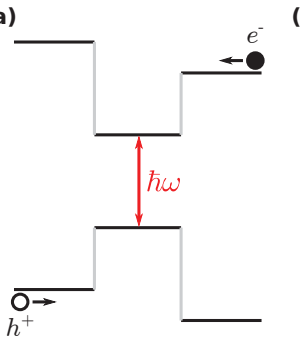

(b)
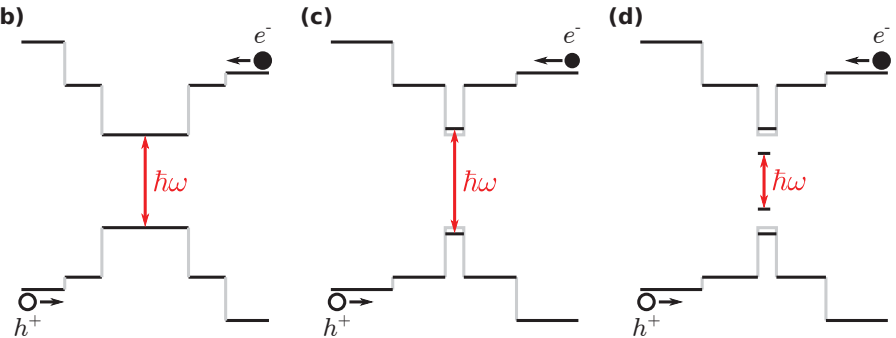

Figure 1.2.: Sketch of the progression of approaches to charge-carrier confinement in semiconductor heterostructures. (a) Double heterostructure, (b) separate confinement heterostructure (SCH), (c) quantum-well heterostructure, (d) dot-in-a-well (DWELL) heterostructure. The optical transitions are denoted by $\hbar \omega$. After [COL12].

optically active region, effectively improving the waveguide properties by confining the light. The resulting five-layer structure is depicted in Fig. 1.2 b. Reducing the active-layer width to a few $\mathrm{nm}$ then leads to the formation of a quantum well (Fig. $1.2 \mathrm{c}$ ). The addition of quantum-dots within the quantum well forms a dot-ina-well (DWELL) structure, with localized quantum-dot states providing the optical transitions, shown in Fig. $1.2 \mathrm{~d}$.

All these steps have subsequently improved the design of semiconductor lasers, leading to the technology that is available today, with highly efficient semiconductor lasers that can be continuously operated at room-temperature and above.

\subsection{Semiconductor Lasers as Dynamical Systems}

Apart from providing a reliable source of constant, coherent, monochromatic light, semiconductor lasers can exhibit a vast variety of different dynamics [LAR10, ERN10b, LUE11b, SOR13]. The simplest model of a semiconductor laser consists of only two equations:

$$
\begin{aligned}
\frac{\mathrm{d}}{\mathrm{d} t} N(t) & =J-2 g\left(N(t)-N_{0}\right) S(t)-\frac{1}{T_{1}} N(t) \\
\frac{\mathrm{d}}{\mathrm{d} t} S(t) & =2 g\left(N(t)-N_{0}\right) S(t)-2 \kappa S(t) .
\end{aligned}
$$

The charge-carrier number $N$ describes the number of electrons and holes in the active laser medium, $S$ the number of photons. The pump process is described by a generalized pump rate $J$, while charge-carrier losses are taken into account by a combined lifetime $T_{1}$. The optical losses are given by the photon loss rate $2 \kappa$.

The term $2 g\left(N-N_{0}\right) S$ accounts for the stimulated emission of photons. The inversion is given by $N-N_{0}$, with $N_{0}$ being the number of charge carriers needed 
to achieve transparency of the medium. The coefficient $g$ describes the linear gain. As soon as the gain $2 g\left(N-N_{0}\right)$ compensates the losses $2 \kappa$, the time-derivative of the photon number becomes positive and the laser light is amplified.

The stimulated emission adds a nonlinearity to the system, which can lead to complex solutions. Semiconductor lasers are known to exhibit a variety of different dynamics. This becomes apparent especially under external perturbations, e.g., of the pump current or by an external light field [TAR95a, WIE05], as well as under optical feedback [HEI01a, SOR13, OTT14]. Here, lasers can exhibit periodic oscillations, multi-stability [GAV97], and deterministic chaos [TAR98a, OHT99].

Solitary semiconductor lasers, due to the two-dimensional phase-space spanned by the inversion and photon number, can at most exhibit oscillations. These damped relaxation oscillations (ROs) can be directly observed and already give an indication about the laser dynamics, as they characterize the laser response to a perturbation. Upon displacement out of its stable lasing state, the laser will perform relaxation oscillations back towards its steady state. The investigation of the relaxation oscillations will therefore be a focus of this thesis.

Due to the prerequisite of charge-carrier inversion in order to obtain optical gain, semiconductor lasers are always operated far from equilibrium. Nevertheless, the charge-carrier distribution in each of the electronic bands will quickly relax towards a quasi-Fermi distribution, with quasi-Fermi energies that differ between conduction and valence bands. While the overall charge-carrier distribution can still be in nonequilibrium, the carriers within the bands can thus be described to be in quasi-equilibrium [SCH87]. The quasi-equilibrium assumptions allow for the description of the charge-carriers with just a single variable $N(t)$, since the charge-carrier distribution can be uniquely calculated from the total charge-carrier number. A description of each $\boldsymbol{k}$-state in the electronic bands is therefore not required.

In this work, we will investigate the cases where the quasi-equilibrium assumption fails. Specifically, we will consider a nonequilibrium between localized quantumdot states and the surrounding continuum states within the same electronic band. It will be shown that this can lead to important differences in the laser dynamics and performance as compared to what would be expected from a quasi-equilibrium description of the quantum-dot laser.

\subsection{Semiconductor Quantum-Dots}

Semiconductor quantum dots can be fabricated in different ways [BIM08a]. The first investigations of nano-scale lattice-matched heterostructures yielded poor results [HIR94a, BIM08], and could not fulfill the predicted performance [ARA82]. The tran- 


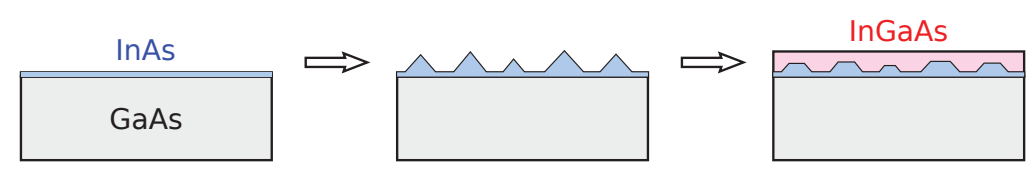

Figure 1.3.: Sketch of the epitaxial Stranski-Krastanov growth of a dot-in-a-well (DWELL) structure. Growth of InAs (blue) on a GaAs layer (light gray) leads to a formation of a wetting layer of only few atomic layers thickness (left). Further growth leads to formation of pyramidal InAs islands (middle). Overgrowth of these islands with InGaAs (red) of few nm height flattens the InAs quantum-dots and embeds them in an InGaAs quantum-well, forming the DWELL structure.

sition to the epitaxial growth of non-lattice-matched semiconductor material on a substrate using molecular beam epitaxy (MBE) or metal-organic chemical vapor deposition (MOCVD) [STR89] lead to a self-organized formation of nano-scale semiconductor islands on top of the substrate layer [PRI95].

The so-called Stranski-Krastanov growth of quantum-dots [STR38, WAL01] is sketched in Fig. 1.3, for InAs quantum dots grown on a GaAs substrate. While at first a very thin InAs layer (wetting layer) is formed on top of the substrate, the strain induced by the mismatch of the lattice constants of the two materials leads to the formation of pyramidal structures. An overgrowth with InGaAs leads to a flattening of the pyramid tops, and additionally creates an InGaAs quantum-well in which the InAs quantum dots are embedded. The resulting dot-in-a-well (DWELL) structure will be considered in all following investigations in this work. A sketch of the energy structure of the electronic states in the DWELL structure is given in Fig. 1.4.

The coupling of the quantum dots to the quantum-well states crucially influences the dynamic properties of quantum-dot optoelectronic devices, as we will show in this thesis. The two-dimensional quantum-well states act as a charge-carrier reservoir for the quantum-dot transitions by means of charge-carrier scattering.

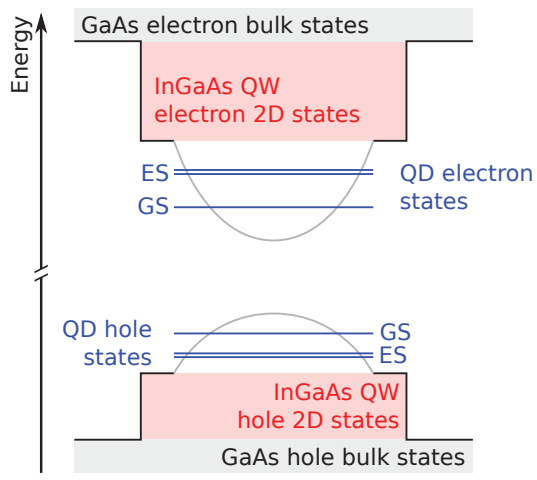

Figure 1.4: Sketch of the energy structure of a dot-in-a-well (DWELL) structure. The localized InAs quantum-dot states (blue), a twofold spin-degenerate ground-state (GS) and a four-fold degenerate excited-state (ES), lie within the band-gap of the InGaAs quantum-well (QW) states (red). The whole structure is embedded within the states of the surrounding GaAs substrate (light gray). 
The finite scattering time of this process can lead to a nonequilibrium between the quantum-dot and surrounding states, which, as we will show, gives rise to unique behavior of quantum-dot devices compared to conventional semiconductor structures. 


\subsection{Outline of the Thesis}

This thesis is organized as follows. In Chapter 2, we will derive a microscopically based balance-equation model for the description of quantum-dot laser devices. At first, we will describe the theoretical framework used to calculate the complex charge-carrier scattering processes in quantum-dot devices. Then, the light-matter interaction will be derived from a Maxwell-Bloch approach, which will be combined with the charge-carrier dynamic equations to formulate a quantum-dot laser rate-equation model. A description of the charge-carrier heating in terms of energy balance equations will be subsequently given, which allows for a dynamic calculation of the quasi-equilibrium temperature in quantum-dot laser devices.

Chapter 3 will investigate the dynamics of quantum-dot lasers with focus on the differences to conventional semiconductor lasers. The laser relaxation oscillations are analyzed in dependence of the charge-carrier scattering rates in Section 3.2. A minimal model for the description of relaxation oscillations will be presented in Section 3.3 and will be subsequently used in an asymptotic analysis. The scatteringrate dependence of the quantum-dot laser modulation response will be given in Section 3.4.

In Section 3.5 we will describe the amplitude-phase coupling in quantum-dot lasers and highlight the differences to the commonly used $\alpha$-factor approach. The applicability of the $\alpha$-factor will also be investigated in Section 3.6, where we discuss the quantum-dot laser dynamics under optical injection. A simplified quantum-dot laser model with optical injection will be presented and implemented for numerical path continuation of bifurcations in Section 3.7. The dynamics of the quantum-dot laser under time-delayed optical feedback will be shown in Section 3.8.

The laser response to generalized external perturbations is the topic of Section 3.9, where we will discuss the frequency-response of the quantum-dot laser to different types of modulation. A summary of the chapter will be given in Section 3.10 .

In Chapter 4 we will investigate the dynamics and performance of quantumdot semiconductor optical amplifiers. An adequate model for the description of the amplifier will be presented in Section 4.2. The amplifier performance under large-signal amplification conditions on ground or excited-state wavelengths will be characterized in Section 4.3. Subsequently, the possibility of dual-state operation of the quantum-dot amplifier will be illuminated in Section 4.4. We will then discuss the occurrence of quantum-coherent effects on a macroscopic scale in quantumdot based amplifiers in Section 4.5, before we give a conclusion of the chapter in Section 4.6. 
A summary of the results of this thesis and an overview about possible further research will be given in Chapter 5 . 


\subsection{Introduction}

Modern semiconductor optical devices can consist of a complex arrangement of several different semiconductor crystal layers. By further processing, the semiconductor structure is then shaped into the desired device geometry. Additional steps, such as planarization and contacting, are then required to yield the final usable device. Naturally, a complete microscopic description of the resulting object in all its degrees of freedom is not tractable. Therefore, a restriction to only few degrees of freedom is required, while still maintaining all necessary aspects determining the system behavior.

In the theoretical description of quantum-dot semiconductor optical devices, this means a restriction to the active region, i.e., the parts where the light-matter interaction occurs, and the immediate surrounding matter. Since the optical interactions between the electric field and charge carriers (electrons and holes) are being considered, dynamic equations for these quantities must be derived.

There exist theoretical models for quantum-dot lasers on varying levels of sophistication. Microscopic models that take into account the exact band structure and many-body interactions [CHO03, ROD05a, FEL06a, GIE07] can describe the complex energy structure of quantum dots very realistically, but these approaches are too complicated to be applied in dynamic problems. On the other hand, simple rateequation models exist [NAD09, ASR10, ERN10a] that can be easily implemented and require little computation power, and often allow for analytical treatment. These models, however, are prone to oversimplification, possibly neglecting important aspects that would lead to different results. In between these two types of approaches there exist multi-rate equation models [TON06, LUE09, GIO12, WAN14b], that take into account the delicate energy structure of quantum-dot active media. These models offer a balance between complexity and practicability. In this spirit, we will develop a quantum-dot laser model that takes into account the most important effects needed to realistically describe the laser behavior, while still being simple enough for thorough dynamic studies. 
This chapter presents the derivation of the microscopically based balance equation model that we will use to model the quantum-dot laser and amplifier devices later on in this work. The charge carrier exchange between the quantum-dots and the surrounding material, forming the carrier reservoir, will be described in terms of microscopically based Boltzmann scattering rates, based on carrier-carrier Augerscattering. The light-matter interaction within the active region will be formulated using a Maxwell-Bloch approach, which we will use to describe the individual contributions of the relevant optical transitions to the optical gain and carrier-induced refractive-index change. Finally, a self-consistent charge-carrier heating model will be presented, in order to dynamically describe the charge-carrier quasi-equilibrium temperature within the quantum-dot device. 


\subsection{Charge-Carrier Scattering in Quantum-Dot Structures}

In electrically operated semiconductor structures, electrons and holes are injected into the medium at the respective contacts. The charge carriers then reach the active region by transport processes due to the applied voltage [SCH87]. In order to reach the energetically lower states, the charge carriers must lose some of their energy. This transfer of energy is realized by means of scattering processes. Here, two different types of charge-carrier scattering can be distinguished: scattering involving only charge carriers and scattering of charge carriers with the environment, most importantly lattice phonons. The scattering mechanisms are illustrated in Fig. 2.1.

Independent of the underlying scattering mechanism, any system will be driven towards an equilibrium state over time. Considering that the fundamental operating principle of lasers - stimulated emission of photons - relies on the inversion of the charge carrier distribution, there always exists a strong nonequilibrium between valence and conduction band electrons. Nevertheless, assuming that the intra-band scattering processes happen on a timescale faster than the inter-band carrier recombination, it is possible to reach a quasi-equilibrium of the electron distributions in each of the bands. As electrons are fermions, this distribution can then be expressed as a quasi-Fermi distribution:

$$
\rho^{\mathrm{eq}}(\varepsilon)=\left[1+\exp \left(\frac{\varepsilon-E_{\mathrm{F}}^{\mathrm{eq}}}{k_{\mathrm{B}} T^{\mathrm{eq}}}\right)\right]^{-1},
$$

with the energy of the corresponding state $\varepsilon$, Boltzmann's constant $k_{\mathrm{B}}$, the quasiequilibrium temperature $T^{\mathrm{eq}}$, and the quasi-Fermi level $E_{\mathrm{F}}^{\mathrm{eq}}$ relative to the corre-

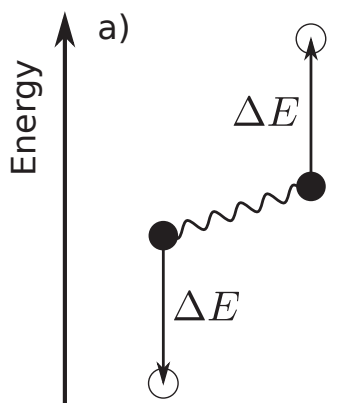

b)

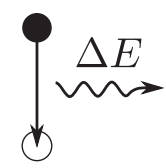

Figure 2.1.: Scattering mechanisms in semiconductor structures. (a) Auger-scattering: an electron scatters into a free energetically lower state under transfer of the energy difference $\Delta E$ to another electron. (b) Carrier-phonon scattering: an electron emits a phonon with energy $\Delta E$ and scatters into a free state $\Delta E$ below its initial state. The reverse process is possible under absorption of a phonon. 
(a)

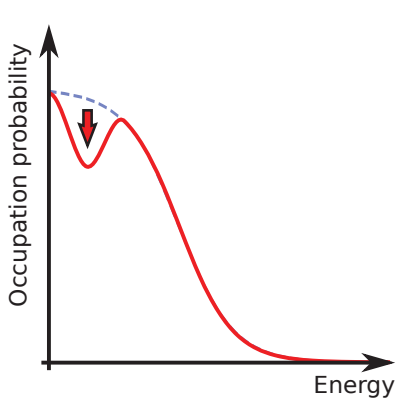

(b)

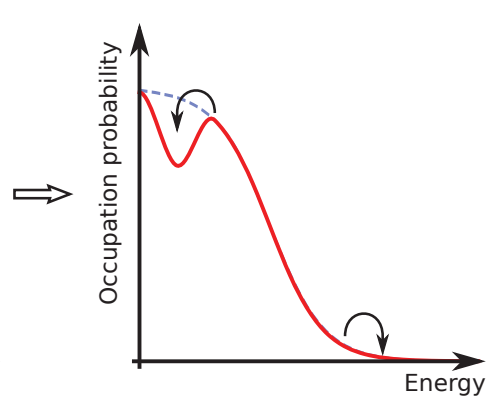

(c)

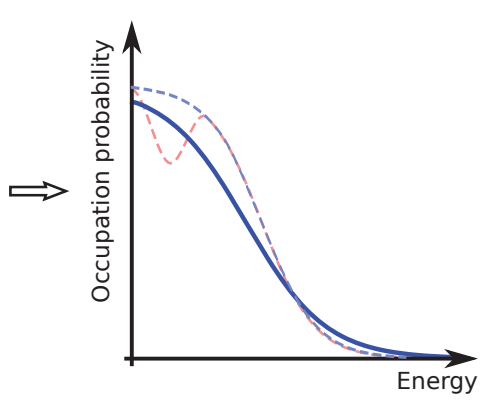

Figure 2.2.: Illustration of carrier heating by Auger-scattering. (a) An initial nonequilibrium electron distribution (red) is created from quasi-equilibrium (light blue dashed). (b) It is then driven towards quasi-equilibrium by Auger-scattering processes. (c) The filling of vacant states at low energy is accompanied by a filling of higher energy states, broadening the resulting quasi-Fermi distribution (blue).

sponding band edge. As the charge carriers are only in quasi-equilibrium, $E_{\mathrm{F}}^{\mathrm{eq}}$ can differ between conduction and valence band.

The most important scattering mechanism for carrier-carrier scattering is the Coulomb interaction between the charged particles. Since in each scattering event the total energy must be conserved, the scattering of one carrier to a lower state must be accompanied by the scattering of the scattering partner to a higher energy with equal energy difference. This type of process is known as Augerscattering [SCH87, USK97a]. Since the total energy of the charge carriers is conserved, Auger-scattering will lead to a change of charge-carrier temperature. A filling of vacant states that lie below the average energy of the electron gas is accompanied by a promotion of other electrons to higher states, such that the total energy is conserved. This effectively broadens the electron distribution, which is equivalent to an increase in temperature and known as Auger-heating [SCH87a, QUA91, QUA93a, BOR97a, FEH02, ACH06a], illustrated in Fig. 2.2.

Apart from the direct interaction between charge carriers, scattering with phonons in the semiconductor lattice is also possible. Here, the charge carriers either absorb or emit energy by interacting with the semiconductor lattice. Contrary to the Auger-scattering mechanism, the scattering with phonons does not conserve the total charge-carrier energy. Thus, a cooling of the charge-carrier distribution towards the lattice temperature is possible.

The theoretical description of the charge-carrier scattering processes can be done on different levels of sophistication. The simplest approach would be a phenomenological description of the scattering by introducing a (constant) scattering time constant at which the charge-carrier population is driven towards a target distribution. On the other end of the spectrum, a microscopic quantum-kinetic description of 
the many-body system allows a realistic modeling of the scattering dynamics. The drawback of such an approach is of course the enormous computational effort in keeping track of such a high-dimensional system.

Here, an intermediate approach will be pursued, such that the numerical handling of the scattering remains manageable, while the underlying physical processes are still accurately taken into account.

\subsubsection{Coulomb-Scattering of Charge Carriers}

The starting point for calculating the carrier-carrier scattering is the system Hamiltonian in second quantization [MAH90],

$$
H_{\mathrm{sys}}=H_{\mathrm{kin}}+H_{\mathrm{C}}=\sum_{\substack{a \\ s}} \varepsilon_{a} a_{a s}^{\dagger} a_{a s}+\frac{1}{2} \sum_{\substack{a b c d \\ s s^{\prime}}} V_{a b c d} a_{a s}^{\dagger} a_{b s^{\prime}}^{\dagger} a_{c s^{\prime}} a_{d s},
$$

where $a_{x}, a_{x}^{\dagger}$ are the electron annihilation and creation operators in the state $x$ with the energy $\varepsilon_{x}$, respectively. The Hamiltonian consists of the kinetic contribution $H_{\text {kin }}$, and the Coulomb-interaction Hamiltonian $H_{\mathrm{C}}$. In the sums $a, b, c, d$ denote all possible electron states, with $s, s^{\prime}$ denoting their spins. The Coulomb interaction matrix element is given by

$$
V_{a b c d}=\iint \mathrm{d} \boldsymbol{r} \mathrm{d} \boldsymbol{r}^{\prime} \phi_{a}^{*}(\boldsymbol{r}) \phi_{b}^{*}\left(\boldsymbol{r}^{\prime}\right) \frac{e_{0}^{2}}{4 \pi \varepsilon_{0} \varepsilon_{\mathrm{bg}}\left|\boldsymbol{r}-\boldsymbol{r}^{\prime}\right|} \phi_{c}\left(\boldsymbol{r}^{\prime}\right) \phi_{d}(\boldsymbol{r}),
$$

with the single-particle wave-functions $\phi_{x}(\boldsymbol{r})$. The vacuum and background permittivity are given by $\varepsilon_{0}$ and $\varepsilon_{\mathrm{bg}}$, respectively, and $-e_{0}$ is the electron charge.

The goal now is to describe the change of the electron probability distribution by Coulomb scattering events. To this end, the change of the occupation probability $\rho_{\nu \sigma} \equiv\left\langle a_{\nu \sigma}^{\dagger} a_{\nu \sigma}\right\rangle$ in a specific state $\nu$ with spin $\sigma$ will be derived. By applying Heisenberg's equation of motion and the commutator relations for fermionic operators, one arrives at:

$$
\begin{aligned}
\frac{\partial}{\partial t} \rho_{\nu \sigma}=\frac{i}{\hbar}\left\langle\left[H_{\mathrm{sys}}, a_{\nu \sigma}^{\dagger} a_{\nu \sigma}\right]\right\rangle & =-\frac{i}{\hbar} \sum_{\substack{b c d \\
s^{\prime}}}\left\langle V_{\nu b c d} a_{\nu \sigma}^{\dagger} a_{b s^{\prime}}^{\dagger} a_{c s^{\prime}} a_{d \sigma}\right\rangle-\text { H.c. } \\
& =\frac{2}{\hbar} \sum_{\substack{b c d \\
s^{\prime}}} \operatorname{Im}\left\langle V_{\nu b c d} a_{\nu \sigma}^{\dagger} a_{b s^{\prime}}^{\dagger} a_{c s^{\prime}} a_{d \sigma}\right\rangle
\end{aligned}
$$

The expectation value of the two-operator expectation value $\rho_{\nu \sigma}$ now couples to a sum of four-operator expectation values. A factorization of these four-operator terms into products of two-operator expectation values leads to the Hartree-Fock approximation of the many-body Coulomb interaction. Within this approxima- 
tion, first-order renormalization effects, including band-gap renormalization and Coulomb enhancement can be described [HAU89, CHO05]. A description of chargecarrier scattering, however, requires the evaluation of higher-order correlations.

The time derivative of the involved four-operator expectation values is again given by Heisenberg's equation of motion:

$$
\begin{aligned}
\frac{\partial}{\partial t}\left\langle a_{\nu \sigma}^{\dagger} a_{b s^{\prime}}^{\dagger} a_{c s^{\prime}} a_{d \sigma}\right\rangle & =\frac{i}{\hbar}\left\langle\left[H_{\mathrm{sys}}, a_{\nu \sigma}^{\dagger} a_{b s^{\prime}}^{\dagger} a_{c s^{\prime}} a_{d \sigma}\right]\right\rangle \\
& =\frac{i}{\hbar}\left(\varepsilon_{\nu}+\varepsilon_{b}-\varepsilon_{c}-\varepsilon_{d}\right)\left\langle a_{\nu \sigma}^{\dagger} a_{b s^{\prime}}^{\dagger} a_{c s^{\prime}} a_{d \sigma}\right\rangle+\frac{i}{\hbar}\left\langle\left[H_{\mathrm{C}}, a_{\nu \sigma}^{\dagger} a_{b s^{\prime}}^{\dagger} a_{c s^{\prime}} a_{d \sigma}\right]\right\rangle .
\end{aligned}
$$

The evaluation of the commutator in the above equation leads to six-operator expectation values, and their time evolution would couple to eight-operator expectation values and so on. Without further approximations, it is therefore not possible to reach a closed set of equations. Thus, in order to get to a closed form, some approximations must be introduced [MAH90]. First, Eq. (2.5) will be solved adiabatically, by assuming a fast evolution to a steady-state, such that $\frac{\partial}{\partial t}\left\langle a_{\nu \sigma}^{\dagger} a_{b s^{\prime}}^{\dagger} a_{c s^{\prime}} a_{d \sigma}\right\rangle=0$. This is the Markov approximation, i.e., the explicit time evolution of $\left\langle a_{\nu \sigma}^{\dagger} a_{b s^{\prime}}^{\dagger} a_{c s^{\prime}} a_{d \sigma}\right\rangle$ is suppressed and its value is solely determined by the current system state. Thus

$$
\left\langle a_{\nu \sigma}^{\dagger} a_{b s^{\prime}}^{\dagger} a_{c s^{\prime}} a_{d \sigma}\right\rangle=-\frac{\left\langle\left[H_{\mathrm{C}}, a_{\nu \sigma}^{\dagger} a_{b s^{\prime}}^{\dagger} a_{c s^{\prime}} a_{d \sigma}\right]\right\rangle}{\left(\varepsilon_{\nu}+\varepsilon_{b}-\varepsilon_{c}-\varepsilon_{d}\right)+i \hbar \gamma},
$$

where an additional phenomenological decay constant $\gamma$ has been introduced. Applying the limit $\gamma \rightarrow 0$ restores the right hand side of Eq. (2.5). This limit is evaluated by applying the Dirac identity

$$
\lim _{\gamma \rightarrow 0^{+}} \frac{1}{x+i \gamma}=\frac{1}{x}-i \pi \delta(x) .
$$

The evaluation of the commutator in Eq. (2.6) contains six-operator expectation values. Instead of deriving equations of motion for these six-operator expressions, they will be factorized into products of two-operator expectation values, neglecting higher order correlations. This means that only terms up to second order in the Coulomb potential will be taken into account. The resulting expectation value can be evaluated to yield

$$
\begin{aligned}
\left\langle\left[H_{\mathrm{C}}, a_{\nu \sigma}^{\dagger} a_{b s^{\prime}}^{\dagger} a_{c s^{\prime}} a_{d \sigma}\right]\right\rangle=\left[V_{\nu b c d}^{*}-V_{\nu b d c}^{*} \delta_{\sigma, s^{\prime}}\right][ & \left(1-\rho_{\nu \sigma}\right)\left(1-\rho_{b s^{\prime}}\right) \rho_{c s^{\prime}} \rho_{d \sigma} \\
& \left.-\rho_{\nu \sigma} \rho_{b s^{\prime}}\left(1-\rho_{c s^{\prime}}\right)\left(1-\rho_{d \sigma}\right)\right] .
\end{aligned}
$$


Inserting this relation into Eq. (2.6), applying Eq. (2.7) and inserting the resulting expression into Eq. (2.4) then gives the following equation:

$$
\begin{aligned}
\left.\frac{\partial}{\partial t} \rho_{\nu \sigma}\right|_{\mathrm{sc}}=\frac{2 \pi}{\hbar} \sum_{\substack{b c d \\
s^{\prime}}} & \operatorname{Re}\left[W_{\nu b c d}\left(W_{\nu b c d}^{*}-W_{\nu b d c}^{*}\right)\right] \delta\left(\varepsilon_{\nu}+\varepsilon_{b}-\varepsilon_{c}-\varepsilon_{d}\right) \\
& \times\left[\left(1-\rho_{\nu \sigma}\right)\left(1-\rho_{b s^{\prime}}\right) \rho_{c s^{\prime}} \rho_{d \sigma}-\rho_{\nu \sigma} \rho_{b s^{\prime}}\left(1-\rho_{c s^{\prime}}\right)\left(1-\rho_{d \sigma}\right)\right],
\end{aligned}
$$

which describes the Coulomb scattering in the second-order Born-Markov approximation [HAU96, NIE04, MAL06, MAJ10]. In the above equation the bare Coulomb potential was replaced with the screened potential $W$, defined via

$$
W\left(\left|\boldsymbol{r}-\boldsymbol{r}^{\prime}\right|\right)=V\left(\left|\boldsymbol{r}-\boldsymbol{r}^{\prime}\right|\right) e^{-\kappa\left|\boldsymbol{r}-\boldsymbol{r}^{\prime}\right|} .
$$

Here, $\kappa$ is the screening wavenumber [HAU84, MAH90], describing the screening of the Coulomb interaction potential by a surrounding charge-carrier plasma, which can be calculated in a self-consistent way [HAU84, HAU89]. The screening becomes very important at elevated charge-carrier densities where the unscreened Coulomb potential would greatly overestimate the interaction between the charge carriers.

The summation terms in Eq. (2.9) describe the simultaneous scattering of $d \leftrightarrow \nu$ and $c \leftrightarrow b$. The delta-function ensures energy conservation, such that the total energy of the final states equals that of the initial states. The first term in the second line describes the probability to find particles in the corresponding initial states $(c, d)$ and vacant spaces in the final states $(\nu, b)$, and the second term describes the corresponding inverse process, leading to a decrease of $\rho_{\nu}$.

Equation (2.9) can be written in form of a Boltzmann equation,

$$
\left.\frac{\partial}{\partial t} \rho(t)\right|_{\mathrm{sc}}=S^{\text {in }}[1-\rho(t)]-S^{\text {out }} \rho(t)
$$

combining the summation terms into an in-scattering rate $S^{\text {in }}$ and a corresponding out-scattering rate $S^{\text {out }}$ which, however, depend on the charge-carrier occupation of all other states.

\section{Scattering Channels in Coupled Quantum-Dot - Quantum-Well Systems}

Equation (2.9) gives the general expression for calculating the Coulomb scattering rates. The sums include all possible states, provided they fulfill the energy conserving $\delta$-function. The given quantum-dot-quantum-well system, however, allows the distinction between qualitatively different scattering processes in order to break up the sums in Eq. (2.9) into different parts which can be handled more easily. 

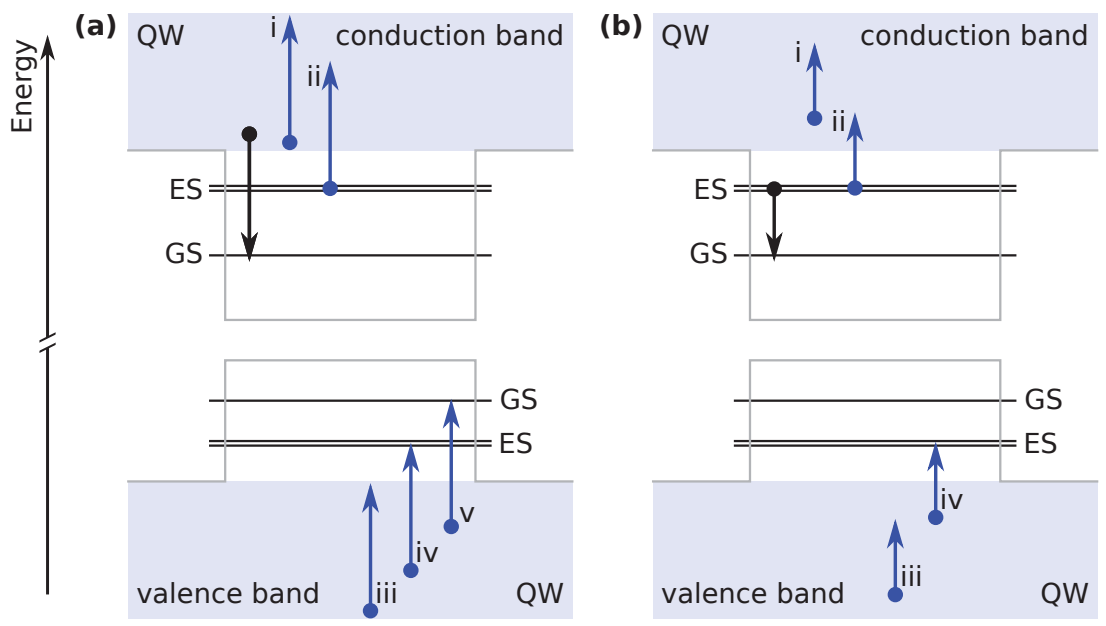

Figure 2.3.: Possible scattering channels in quantum-dot-quantum-well systems. (a) Direct capture into the quantum dot (QD) ground state, (b) Intra-dot relaxation from excited state (ES) to ground-state (GS). The considered electron scattering process is shown by the black arrow, with the blue arrows denoting the possible simultaneous scattering of the Auger-electron to a vacant state: (i) quantum-well (QW) intra-band electron transition, (ii) electron escape from ES to quantum well. The corresponding mixed processes are shown in (iii) and (iv), where the Auger-electron is in the valence band, (v) showing a capture of a valence electron to the GS. For all processes shown, the corresponding reverse scattering is also possible. Not shown is the direct capture into the quantum-dot excited state, analogous to (a).

Throughout this work, quantum-dots embedded in a quantum-well (dot-in-awell, DWELL structure) are considered, with two localized quantum-dot states in both the conduction and valence band. Therefore, two general charge-carrier scattering processes can be distinguished: Capture of a quantum-well electron into a confined quantum-dot state, and intra-dot electron relaxation, with their respective inverse processes. This is illustrated in Fig. $2.3 \mathrm{a}$ and b, respectively. The accompanying Auger-process can involve either quantum-well states only, or transitions between quantum-well and other quantum-dot states. Note that depending on the energy difference, not all these scattering channels are possible. For example, in the depicted case of the intra-dot relaxation in Fig. $2.3 \mathrm{~b}$, the Auger-transition in the valence band from the quantum well to the ground state is not possible, as it would violate energy conservation. The possible scattering processes contributing to the total scattering rate thus strongly depend on the exact energy scheme of the quantum-dot-quantum-well system. Note that throughout this work impact ionization and Auger-assisted recombination, i.e. the direct scattering between conduction and valence bands, is not considered. 
Following the above discussion, the scattering dynamics of the quantum-dot states are rewritten as

$$
\begin{aligned}
\left.\frac{\partial \rho_{c, \mathrm{GS}}}{\partial t}\right|_{s c}= & S_{c, \mathrm{GS}}^{\mathrm{cap}, \mathrm{in}}\left(\left\{\rho_{\mathrm{QW}}\right\}\right)\left(1-\rho_{c, \mathrm{GS}}\right)-S_{c, \mathrm{GS}}^{\mathrm{cap}, \mathrm{out}}\left(\left\{\rho_{\mathrm{QW}}\right\}\right) \rho_{c, \mathrm{GS}} \\
& +S_{c, \mathrm{GS}}^{\mathrm{rel}, \mathrm{in}}\left(\left\{\rho_{\mathrm{QW}}\right\}\right) \rho_{c, \mathrm{ES}}\left(1-\rho_{c, \mathrm{GS}}\right)-S_{c, \mathrm{GS}}^{\mathrm{rel}, \mathrm{out}}\left(\left\{\rho_{\mathrm{QW}}\right\}\right)\left(1-\rho_{c, \mathrm{ES}}\right) \rho_{c, \mathrm{GS}}, \\
\left.\frac{\partial \rho_{c, \mathrm{ES}}}{\partial t}\right|_{s c}= & S_{c, \mathrm{ES}}^{\mathrm{cap}, \mathrm{in}}\left(\left\{\rho_{\mathrm{QW}}\right\}\right)\left(1-\rho_{c, \mathrm{ES}}\right)-S_{c, \mathrm{ES}}^{\mathrm{cap}, \mathrm{out}}\left(\left\{\rho_{\mathrm{QW}}\right\}\right) \rho_{c, \mathrm{ES}} \\
& +S_{c, \mathrm{ES}}^{\mathrm{rel}, \mathrm{in}}\left(\left\{\rho_{\mathrm{QW}}\right\}\right) \rho_{c, \mathrm{GS}}\left(1-\rho_{c, \mathrm{ES}}\right)-S_{c, \mathrm{ES}}^{\mathrm{rel}, \mathrm{out}}\left(\left\{\rho_{\mathrm{QW}}\right\}\right)\left(1-\rho_{c, \mathrm{GS}}\right) \rho_{c, \mathrm{ES}} .
\end{aligned}
$$

Here, $\rho_{c}$ denote the electron occupation probabilities of the conduction band states. For valence band states, analogous equations can be written down (subscript $v$ ). The scattering rates $S^{\text {cap,in }}$ denote the direct capture of quantum-well electrons into the quantum-dot states, $S^{\text {rel,in }}$ the intra-dot relaxation between the quantumdot states, with $S^{\text {out }}$ being the scattering rate of the respective reverse processes. We can identify the relaxation processes of the excited state with the GS terms,

$$
\begin{aligned}
S_{c, \mathrm{ES}}^{\mathrm{rel}, \mathrm{in}}\left(\left\{\rho_{\mathrm{QW}}\right\}\right) & =-\frac{1}{2} S_{c, \mathrm{GS}}^{\mathrm{rel}, \mathrm{out}}\left(\left\{\rho_{\mathrm{QW}}\right\}\right), \\
S_{c, \mathrm{ES}}^{\mathrm{rel}, \mathrm{out}}\left(\left\{\rho_{\mathrm{QW}}\right\}\right) & =-\frac{1}{2} S_{c, \mathrm{GS}}^{\mathrm{rel}, \mathrm{in}}\left(\left\{\rho_{\mathrm{QW}}\right\}\right),
\end{aligned}
$$

with a factor $\frac{1}{2}$ compared to the ground-state contribution, due to its two-fold degeneracy. All scattering rates in above equations depend on the whole quantumwell distribution in both bands, denoted by $\left\{\rho_{\mathrm{QW}}\right\}$.

\subsubsection{Electron-Hole Picture}

It is customary in semiconductor physics to describe the charge carriers in the electron-hole picture, where, starting from a completely filled valence band, an unoccupied state is described as a positively charged hole. The charge-carrier occupations in the conduction and valence bands can then be given in terms of electron and hole populations:

$$
\begin{aligned}
\rho_{e} & \equiv \rho_{c} \\
\rho_{h} & \equiv 1-\rho_{v} .
\end{aligned}
$$

This description leads to a reversed energy axis for holes, leading to a positive effective hole mass. The expressions for the scattering rates is formally identical to the electron picture. The sums in Eq. (2.9), however, now run over both electron 
and hole states. Using $b \in\{e, h\}$ to distinguish electron and hole states, the scattering dynamics can be written as:

$$
\begin{aligned}
\left.\frac{\partial \rho_{b, \mathrm{GS}}}{\partial t}\right|_{\mathrm{sc}}= & S_{b, \mathrm{GS}}^{\mathrm{cap}, \mathrm{in}}\left(\left\{\rho_{\mathrm{QW}}\right\}\right)\left[1-\rho_{b, \mathrm{GS}}\right]-S_{b, \mathrm{GS}}^{\mathrm{cap}, \mathrm{out}}\left(\left\{\rho_{\mathrm{QW}}\right\}\right) \rho_{b, \mathrm{GS}} \\
& +S_{b}^{\mathrm{rel}, \mathrm{in}}\left(\left\{\rho_{\mathrm{QW}}\right\}\right) \rho_{b, \mathrm{ES}}\left[1-\rho_{b, \mathrm{GS}}\right]-S_{b}^{\mathrm{rel}, \mathrm{out}}\left(\left\{\rho_{\mathrm{QW}}\right\}\right)\left[1-\rho_{b, \mathrm{ES}}\right] \rho_{b, \mathrm{GS}} \\
\left.\frac{\partial \rho_{b, \mathrm{ES}}}{\partial t}\right|_{s c}= & S_{b, \mathrm{ES}}^{\mathrm{cap}, \mathrm{in}}\left(\left\{\rho_{\mathrm{QW}}\right\}\right)\left[1-\rho_{b, \mathrm{ES}}\right]-S_{b, \mathrm{ES}}^{\mathrm{cap}, \mathrm{out}}\left(\left\{\rho_{\mathrm{QW}}\right\}\right) \rho_{b, \mathrm{ES}} \\
& -\frac{1}{2}\left[S_{b}^{\mathrm{rel}, \mathrm{in}}\left(\left\{\rho_{\mathrm{QW}}\right\}\right) \rho_{b, \mathrm{ES}}\left[1-\rho_{b, \mathrm{GS}}\right]-S_{b}^{\mathrm{rel}, \mathrm{out}}\left(\left\{\rho_{\mathrm{QW}}\right\}\right)\left[1-\rho_{b, \mathrm{ES}}\right] \rho_{b, \mathrm{GS}}\right] .
\end{aligned}
$$

One thing to note is that the capture of valence band electrons into the localized quantum-dot states now corresponds to the escape of holes from the quantum-dots, thus reversing the interpretation of in and out-scattering processes in the valence band. The formal structure of the above equation is nevertheless conserved.

\subsubsection{Detailed Balance}

The derived scattering expressions so far only describe the dynamics of quantumdot states and their interaction with the quantum-well charge carriers. The dynamics of quantum-well carriers can in principle be expressed by Eq. (2.9) as well. However, this would require resolving all quantum-well states and tracking their population distribution in time, which greatly increases the dimensionality of the system state. This problem can be resolved by assuming a specific distribution of the carrier population within the quantum-well.

The intra-band scattering between quantum-well states is typically in the order of $\approx 100 \mathrm{fs}$ [BIN92a, TRA92, CAM96, KAN96b, BAN98]. As long as this scattering is faster than the charge-carrier exchange between the quantum well and quantum-dots, the quantum well can be assumed to be in quasi-equilibrium with good accuracy:

$$
\rho_{b, \mathrm{QW}}\left(\varepsilon_{b, \boldsymbol{k}}^{2 \mathrm{D}}\right) \approx f\left(\varepsilon_{b, \boldsymbol{k}}^{2 \mathrm{D}}, E_{\mathrm{F}, b}^{\mathrm{eq}}, T^{\mathrm{eq}}\right) \equiv\left[1+\exp \left(\frac{\varepsilon_{b, \boldsymbol{k}}^{2 \mathrm{D}}-E_{\mathrm{F}, b}^{\mathrm{eq}}}{k_{\mathrm{B}} T^{\mathrm{eq}}}\right)\right]^{-1},
$$

with the corresponding single-particle energies $\varepsilon_{b, k}^{2 \mathrm{D}}$ and the quasi-Fermi level $E_{\mathrm{F}, b}^{\mathrm{eq}}$. From this quasi-Fermi distribution the 2D-charge-carrier density $w_{b}$ in the QWs can be calculated, by taking the density of states in the quantum well as

$$
\mathcal{D}_{b}(E)=D_{b} \Theta\left(E-E_{b, 0}^{\mathrm{QW}}\right)=\frac{m_{b}^{*}}{\pi \hbar^{2}} \Theta\left(E-E_{b, 0}^{\mathrm{QW}}\right),
$$


under the assumption that the quantum-well sub-band spacing is large enough that only the lowest sub-band needs to be taken into account. The energy $E_{b, 0}^{\mathrm{QW}}$ is the corresponding quantum-well band edge and $\Theta$ is the Heaviside function. The quantum-well charge-carrier density can then be written as

$$
\begin{aligned}
w_{b} & =\frac{2}{A_{\mathrm{act}}} \sum_{k^{2 \mathrm{D}}}\left[1+\exp \left(\frac{\varepsilon_{b, k}^{2 \mathrm{D}}-E_{\mathrm{F}, b}^{\mathrm{eq}}}{k_{\mathrm{B}} T^{\mathrm{eq}}}\right)\right]^{-1} \\
& =\int_{-\infty}^{\infty} \mathrm{d} \varepsilon_{b, k}^{2 \mathrm{D}} \mathcal{D}_{b}\left(\varepsilon_{b, k}^{2 \mathrm{D}}\right)\left[1+\exp \left(\frac{\varepsilon_{b, k}^{2 \mathrm{D}}-E_{\mathrm{F}, b}^{\mathrm{eq}}}{k_{\mathrm{B}} T^{\mathrm{eq}}}\right)\right]^{-1} \\
& =D_{b} k_{\mathrm{B}} T^{\mathrm{eq}} \log \left[1+\exp \left(\frac{E_{\mathrm{F}, b}^{\mathrm{eq}}-E_{b, 0}^{\mathrm{QW}}}{k_{\mathrm{B}} T^{\mathrm{eq}}}\right)\right],
\end{aligned}
$$

where the sum over all quantum-well $\boldsymbol{k}$-states was expressed as the integral over the charge-carrier energy. $A_{\text {act }}$ is the active region in-plane area, with the factor 2 accounting for spin degeneracy. By inverting the above expression, the quasi-Fermi level $E_{\mathrm{F}, b}^{\mathrm{eq}}$ can be expressed in terms of the charge-carrier density in the quantum well,

$$
E_{\mathrm{F}, b}^{\mathrm{eq}}=E_{b, 0}^{\mathrm{QW}}+k_{\mathrm{B}} T^{\mathrm{eq}} \log \left[\exp \left(\frac{w_{b}}{D_{b} k_{\mathrm{B}} T^{\mathrm{eq}}}\right)-1\right]
$$

Thus, the quantum-well charge-carrier population can be expressed as a function of the carrier density and the quasi-equilibrium temperature:

$$
\rho_{b, \mathrm{QW}}\left(\varepsilon_{b, \boldsymbol{k}}^{2 \mathrm{D}}\right) \equiv \rho_{b, \mathrm{QW}}\left(\varepsilon_{b, \boldsymbol{k}}^{2 \mathrm{D}}, w_{b}, T^{\mathrm{eq}}\right)=\left[1+\exp \left(\frac{\varepsilon_{b, \boldsymbol{k}}^{2 \mathrm{D}}-E_{\mathrm{F}, b}^{\mathrm{eq}}\left(w_{b}, T^{\mathrm{eq}}\right)}{k_{\mathrm{B}} T^{\mathrm{eq}}}\right)\right]^{-1}
$$

By entering this relation into the expressions for the scattering rates Eq. (2.9), also the individual scattering rates can be expressed as functions of only the $2 \mathrm{D}$ chargecarrier densities $w_{b}$ and their quasi-equilibrium temperature $T^{e q}$, eliminating the need to keep track of the microscopic carrier population distributions.

Furthermore, it is now possible to relate the in and out-scattering rates of a given scattering process to each other [SCH87, LUE09]. The out-scattering contribution in Eq. (2.9) is equivalent to the in-scattering contribution under the replacement $\rho \rightarrow 1-\rho$, which for the quantum well in quasi-equilibrium can be expressed as

$$
1-\rho_{\mathrm{QW}}\left(\varepsilon_{b, k}^{2 \mathrm{D}}\right)=\rho_{\mathrm{QW}}\left(\varepsilon_{b, k}^{2 \mathrm{D}}\right) \exp \left(\frac{\varepsilon_{b, \boldsymbol{k}}^{2 \mathrm{D}}-E_{\mathrm{F}, b}^{\mathrm{eq}}\left(w_{b}, T^{\mathrm{eq}}\right)}{k_{\mathrm{B}} T^{\mathrm{eq}}}\right) .
$$


For the quantum-dot scattering processes the out-scattering rates can thus be written as [LUE11a]:

$$
\begin{aligned}
S_{b, m}^{\mathrm{cap}, \text { out }}\left(w_{e}, w_{h}, T^{\mathrm{eq}}\right) & =S_{b, m}^{\mathrm{cap}, \mathrm{in}}\left(w_{e}, w_{h}, T^{\mathrm{eq}}\right) \exp \left(\frac{\varepsilon_{b, m}^{\mathrm{QD}}-E_{\mathrm{F}, b}^{\mathrm{eq}}}{k_{\mathrm{B}} T^{\mathrm{eq}}}\right) \\
S_{b}^{\mathrm{rel}, \text { out }}\left(w_{e}, w_{h}, T^{\mathrm{eq}}\right) & =S_{b}^{\mathrm{rel}, \mathrm{in}}\left(w_{e}, w_{h}, T^{\mathrm{eq}}\right) \exp \left(\frac{\varepsilon_{b, \mathrm{GS}}^{\mathrm{QD}}-\varepsilon_{b, \mathrm{ES}}^{\mathrm{QD}}}{k_{\mathrm{B}} T^{\mathrm{eq}}}\right),
\end{aligned}
$$

where $\varepsilon_{b, m}^{\mathrm{QD}}$ denotes the energy of the localized quantum-dot state, with $m \in$ \{GS, ES\} distinguishing between ground and excited state. The out-scattering of charge-carriers thus becomes more probable at elevated charge-carrier temperatures [URA02, ROS09a]. Note that in the derivation of above expressions, only a quasi-equilibrium within the quantum well must be assumed without making assumptions about the quantum-dot occupations. Equation (2.22) is therefore valid also in nonequilibrium situations between quantum dot and quantum well.

\subsubsection{Carrier-Phonon Scattering}

Apart from the direct interaction of the charge carriers moving along the semiconductor lattice, an interaction with the lattice itself is possible. This interaction occurs by the excitation of phonons, the quanta of lattice atom oscillations. In polar semiconductors, such as GaAs, this displacement of the ionic lattice atoms leads to the build-up of a polarization field. Charge carriers can thus couple to phonons in a polar semiconductor via the Coulomb interaction.

Two different important types of phonons must be distinguished: longitudinal optical (LO) phonons and longitudinal acoustic (LA) phonons. The difference between the two varieties lies in their dispersion relation $\omega(\boldsymbol{k})$. LO phonons have a constant energy around the $\Gamma$-point (wave vector $\boldsymbol{k}=0$ ) and are thus dispersionless (for small $\boldsymbol{k}$ ), while LA phonons show a nearly linear dispersion relation:

$$
\omega_{\mathrm{LO}}(\boldsymbol{k}) \approx \text { const. } \quad \omega_{\mathrm{LA}}(\boldsymbol{k}) \approx v_{s}|k|,
$$

where $v_{s}$ is the speed of sound. The different dispersion relations critically influence the scattering dynamics of charge carriers with these types of phonons. Since both energy and momentum must be conserved in scattering events, LA phonons only allow for an efficient scattering under the condition that both the energy and momentum difference between the initial and final states of a given scattering event match that of the phonon. This greatly limits the number of possible scattering partners. The scattering with LO phonons, on the other hand, is nearly independent of the momenta of a given initial and final state, as long as their energy 
difference matches the LO phonon energy $\hbar \omega_{\mathrm{LO}}$. While the scattering to transversal optical (TO) phonons is in principle also possible, the transversal oscillation results in a much smaller total electric field strength and thus to only little interaction with electrons [MAH90].

The quantum-mechanical description of the carrier-phonon interaction is commonly expressed by the Fröhlich electron-phonon coupling Hamiltonian [MAH90]:

$$
H_{\mathrm{cp}}=\sum_{\substack{a b q \\ s}} \hbar g_{\boldsymbol{q}}^{a b} a_{a}^{\dagger} a_{b}\left(b_{\boldsymbol{q}}+b_{-q}^{\dagger}\right) .
$$

This Hamiltonian describes the transition between electronic states $b \rightarrow a$ under either the emission of a LO phonon with wave vector $\boldsymbol{q}$ or the absorption of one with wave vector $\boldsymbol{- q}$. The coupling matrix element for these transitions is given by

$$
\begin{aligned}
g_{\boldsymbol{q}}^{a b} & =\left\langle a\left|e^{i \boldsymbol{q} \boldsymbol{r}}\right| b\right\rangle g_{\boldsymbol{q}} \\
g_{\boldsymbol{q}} & =\left[\frac{\omega_{\mathrm{LO}} W_{q}^{3 D}}{2 \hbar}\left(\frac{1}{\varepsilon_{\infty}^{\mathrm{bg}}}-\frac{1}{\varepsilon_{0}^{\mathrm{bg}}}\right)\right]^{\frac{1}{2}} .
\end{aligned}
$$

Here, $\varepsilon_{0}^{\mathrm{bg}}$ and $\varepsilon_{\infty}^{\mathrm{bg}}$ describe the static and high-frequency background permittivity of the medium, respectively. $W_{q}^{3 D}$ is the Fourier-transform of the statically screened three-dimensional Coulomb interaction potential,

$$
W_{q}^{3 D}=\frac{e_{0}{ }^{2}}{V \varepsilon_{0} \varepsilon_{\mathrm{bg}}\left(q^{2}+\kappa^{2}\right)},
$$

with the normalization volume $V$, and the screening wavenumber $\kappa$.

Following a similar approach as for the carrier-carrier scattering, we can derive an expression for the charge-carrier scattering by carrier-phonon scattering [CHO99, NIE04]:

$$
\begin{array}{r}
\left.\frac{\partial}{\partial t} \rho_{\nu \sigma}\right|_{c p}=2 \pi \sum_{a \boldsymbol{q}}\left|g_{\boldsymbol{q}}^{a \nu}\right|^{2}\left\{\delta_{\nu, a+\mathrm{LO}}\left[\left(1-\rho_{\nu \sigma}\right) \rho_{a \sigma} n_{\mathrm{ph}, \boldsymbol{q}}-\rho_{\nu \sigma}\left(1-\rho_{a \sigma}\right)\left(n_{\mathrm{ph}, \boldsymbol{q}}+1\right)\right]\right. \\
\left.\delta_{\nu, a-\mathrm{LO}}\left[\left(1-\rho_{\nu \sigma}\right) \rho_{a \sigma}\left(n_{\mathrm{ph}, \boldsymbol{q}}+1\right)-\rho_{\nu \sigma}\left(1-\rho_{a \sigma}\right) n_{\mathrm{ph}, \boldsymbol{q}}\right]\right\},
\end{array}
$$

where $\delta_{\nu, a \pm \mathrm{LO}} \equiv \delta\left(\varepsilon_{\nu}-\left(\varepsilon_{a} \pm \hbar \omega_{\mathrm{LO}}\right)\right)$ describe the possible situations where the state $\nu$ lies $\hbar \omega_{\mathrm{LO}}$ above $(+)$ or below (-) the state $a$. The terms proportional to the phonon number $n_{\mathrm{ph}, \boldsymbol{q}}$ account for the processes where a phonon is absorbed, while those including $\left(n_{\mathrm{ph}, \boldsymbol{q}}+1\right)$ account for stimulated and spontaneous emission of a phonon. 
Phonon scattering plays an important role for intra-band relaxation processes in bulk and quantum-well structures [ASA89] as well as charge-carrier capture processes into quantum wells from the surrounding bulk semiconductor. The continuous density of states of charge carriers in these systems allow for an efficient scattering with phonons due to many possible transitions matching the LO phonon energy. A cooling of the carrier distribution through emission of phonons is therefore possible and occurs typically on timescales $\approx 5-10 \mathrm{ps}$ in InGaAs quantum-wells [WEN06, VAL13].

For scattering processes involving quantum-dot states, on the other hand, the $\delta$-function in Eq. (2.28) greatly limits the scattering efficiency. As the quantumdot levels are at a discrete distance from the quantum-well band edges, they can couple only to a single charge-carrier energy level within the quantum well by LO phonon scattering. In the case that the quantum-dot localization energy exceeds the LO phonon energy, scattering between the quantum dot and quantum well by phonons becomes possible only by multi-phonon processes, which have a much lower probability to occur, especially at low temperatures [INO92, FEL01]. For intra-dot carrier relaxation processes, effective scattering by LO phonons becomes possible only when the quantum-dot level spacing is an integer multiple of the LO phonon energy, which should apply only for a vanishingly small number of QDs in a given semiconductor structure.

However, it has been shown that the Markov approximation, leading to the emergence of the energy conserving $\delta$-function in the scattering rates, can underestimate its actual value [SEE05, SEE09, STE12]. This is due to a broadening of the transition probabilities in energy space by non-Markovian dynamics, which makes scattering possible also for energy differences not matching the LO phonon energy exactly. In [STE13, SCH13k] it is shown that the interplay between Coulomb and carrier-phonon interaction can lead to an enhancement of scattering rates, with the total rate being higher than the sum of the individual processes. This effect becomes especially prevalent for intra-dot relaxation processes, leading to efficient scattering only weakly dependent on the spacing of quantum-dot energy levels. The chargecarrier capture into QDs, on the other hand, was shown to be well described by carrier-carrier scattering for large enough carrier densities, as they are commonly encountered in quantum-dot electro-optical structures.

In the remainder of this work, we therefore neglect the contribution of the carrierphonon interaction on the quantum-dot scattering dynamics, in order to maintain computational efficiency. The Coulomb carrier-carrier scattering will be taken into account as the dominant scattering process, which describes the charge-carrier dynamics sufficiently accurately while still allowing for a dynamical analysis of the device behavior. 


\subsection{Light-Matter Interaction}

So far we have derived equations describing the charge-carrier dynamics in the quantum-dot optical structure due to scattering events. In this section, the interaction of the semiconductor medium with light will be derived. The description of light can be done within a semi-classical framework, where the light field itself is described by Maxwell's equations using classical fields. This treatment is often sufficient to describe all important effects governing the behavior of macroscopic semiconductor devices [HAK83a, CHO99].

A fully quantum-mechanical description of the light and its interaction with the semiconductor medium can, however, lead to deviations from the semi-classical treatment under certain conditions. This becomes especially evident in the case when only a few quantum-dots or photons are involved in the lasing process [RIT10, GIE11]. Then, the non-classical light output from quantum-dots can be used, e.g., for creation of entangled photons [BEN00a] or single-photon emission [UNR12, CAL13]. When discussing optical feedback of few-photon quantum-dot lasers, non-classical effects were also found to arise, characterized by a "bunching" of photons [ALB11, SCH13g], that is unaccounted for in semi-classical models. In this work, we will limit ourselves to macroscopic scales, and thus classical light states. A semi-classical description of the light-matter interaction is thus sufficient.

\subsubsection{Electric Field Dynamics}

The starting point for the classical description of the electric field dynamics are Maxwell's equations for the dielectric displacement field $\boldsymbol{D}$, the magnetic field $\boldsymbol{B}$, the electric field $\mathcal{E}$, and the magnetizing field $\boldsymbol{H}$ :

$$
\begin{aligned}
\nabla \cdot \boldsymbol{D}(\boldsymbol{r}, t) & =\rho(\boldsymbol{r}, t) \\
\nabla \cdot \boldsymbol{B}(\boldsymbol{r}, t) & =0 \\
\nabla \times \mathcal{E}(\boldsymbol{r}, t) & =-\frac{\partial}{\partial t} \boldsymbol{B}(\boldsymbol{r}, t) \\
\nabla \times \boldsymbol{H}(\boldsymbol{r}, t) & =\boldsymbol{j}+\frac{\partial}{\partial t} \boldsymbol{D}(\boldsymbol{r}, t),
\end{aligned}
$$

with the free charge-carrier density $\rho$, the free current density $\boldsymbol{j}$ and the electric displacement and magnetizing field given by

$$
\begin{aligned}
& \boldsymbol{D}(\boldsymbol{r}, t)=\varepsilon_{0} \mathcal{E}(\boldsymbol{r}, t)+\mathcal{P}(\boldsymbol{r}, t), \\
& \boldsymbol{H}(\boldsymbol{r}, t)=\frac{1}{\mu_{0}}(\boldsymbol{B}(\boldsymbol{r}, t)-\boldsymbol{M}(\boldsymbol{r}, t)),
\end{aligned}
$$


respectively. $\mathcal{P}$ and $\boldsymbol{M}$ are the medium polarization and magnetization, respectively. Assuming vanishing free carrier density and current, as well as a nonmagnetizable medium, Maxwell's equations can be combined to yield the wave equation for the electric field

$$
\varepsilon_{0} \mu_{0} \frac{\partial^{2}}{\partial t^{2}} \mathcal{E}(\boldsymbol{r}, t)-\Delta \mathcal{E}(\boldsymbol{r}, t)=-\mu_{0} \frac{\partial^{2}}{\partial t^{2}} \mathcal{P}(\boldsymbol{r}, t),
$$

with the Laplace operator $\Delta$. Applying the slowly varying wave approximation, one reaches the time derivation of the slowly varying electric field envelope

$$
\frac{\partial}{\partial t} E(\boldsymbol{r}, t)=\frac{i \omega}{2 \varepsilon_{0} \varepsilon_{\mathrm{bg}}} P(\boldsymbol{r}, t) .
$$

where $E, P$ are defined via

$$
\begin{aligned}
\mathcal{E}(\boldsymbol{r}, t) & =\frac{1}{2}\left(E(\boldsymbol{r}, t) e^{i(\boldsymbol{k} \cdot \boldsymbol{r}-\omega t)}+\text { c.c. }\right) \hat{\boldsymbol{e}} \\
\mathcal{P}(\boldsymbol{r}, t) & =\frac{1}{2}\left(P(\boldsymbol{r}, t) e^{i(\boldsymbol{k} \cdot \boldsymbol{r}-\omega t)}+\text { c.c. }\right) \hat{\boldsymbol{e}}+\varepsilon_{0} \chi_{\mathrm{bg}} \mathcal{E}(r, t),
\end{aligned}
$$

with a carrier frequency $\omega$, and a unit vector $\hat{\boldsymbol{e}}$ giving the electric field polarization. The real background susceptibility $\chi_{\mathrm{bg}}$ accounts for the linear response of the background medium polarization to the incident electric field and yields the background susceptibility $\varepsilon_{\mathrm{bg}} \equiv\left(1+\chi_{\mathrm{bg}}\right)$, leading to the background refractive index $n_{\mathrm{bg}}=\sqrt{\varepsilon_{\mathrm{bg}}}$. The remaining contribution from $P(\boldsymbol{r}, t)$ contains the response of the active medium, which in general cannot be assumed to be linear in $\mathcal{E}$, and can also have an imaginary part, leading to absorption or amplification of the electric field. The fields are expanded in terms of plane waves with the wave vector $\boldsymbol{k}$, where $|\boldsymbol{k}|=\frac{\omega n_{\mathrm{bg}}}{c_{0}}$. The slowly varying envelope functions $E, P$ are in general complex and thus include both the spatial amplitude as well as the phase profile of the fields.

It is often customary to separate the spatial dependence of the electric field from its time dependence,

$$
E(\boldsymbol{r}, t)=E(t) u_{E}(\boldsymbol{r}),
$$

thus assuming a time-independent electric field profile in space. In a cavity, $u(\boldsymbol{r})$ describes the main cavity mode profile. Equation (2.36) then corresponds to the single-mode approximation. In general optical cavities allow for a higher number of possible modes, especially in Fabry-Perot type cavities. When a description of multi-mode dynamics is required, Eq. (2.36) can be extended to a linear superposition of the different cavity modes [LEN14]. The assumption of a single mode is nevertheless justified in Fabry-Perot microcavity devices, where the mode spacing between longitudinal modes is very large, as well as in distributed feedback (DFB) 


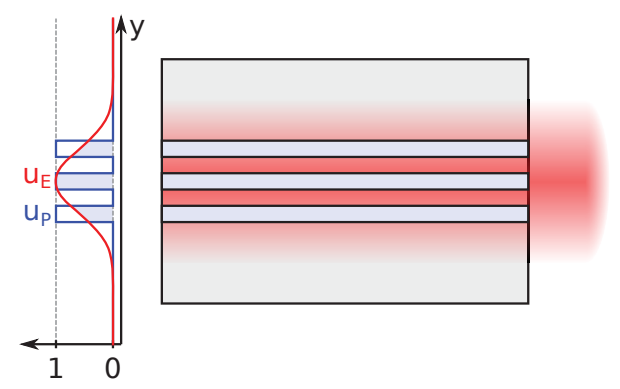

Figure 2.4.: Illustration of the geometric confinement factor. The optical mode profile $u_{E}(\boldsymbol{r})$ (red) often extends beyond the active medium (light blue). The confinement factor describes the overlap of the mode with the active medium distribution $u_{P}(\boldsymbol{r})$ (blue).

devices, where optical modes other than the fundamental cavity mode are suppressed [KOG72, MIN97b, GIO11]. In the remainder of this work we therefore assume a single-mode electric field profile.

The expansion of the electric field in terms of a single mode simplifies the description of the electric field dynamics by eliminating its spatial dependence. The polarization field in the active medium can be expanded in a similar way as Eq. (2.36),

$$
P(\boldsymbol{r}, t)=P(t) u_{P}(\boldsymbol{r}) u_{E}(\boldsymbol{r}),
$$

under the assumption that the polarization amplitude is in first order proportional to the electric field amplitude and thus to the electric field mode profile. The spatial polarization profile $u_{P}(\boldsymbol{r}) \in \mathbb{R}$ is defined by the active medium geometry. In general, the spatial extension of electric and polarization field are different, with the electric field often spanning over a larger volume.

Multiplying Eq. (2.33) with $E^{*}(z, t)$, adding its complex conjugate and integrating over $r$ yields

$$
\begin{aligned}
\int \mathrm{d}^{3} r & E^{*}(z, t) \frac{\partial}{\partial t} E(z, t)+\text { c.c. } \\
& =\frac{\partial}{\partial t}|E(t)|^{2} \int \mathrm{d}^{3} r\left|u_{E}(\boldsymbol{r})\right|^{2} \\
& =\frac{\omega}{2 \varepsilon_{0} \varepsilon_{\mathrm{bg}}}\left[i E^{*}(t) P(t)+\text { c.c. }\right] \int \mathrm{d}^{3} r\left|u_{E}(\boldsymbol{r})\right|^{2} u_{P}(\boldsymbol{r}),
\end{aligned}
$$

which we write as

$$
\frac{\partial}{\partial t}|E(t)|^{2}=\Gamma \frac{\omega}{2 \varepsilon_{0} \varepsilon_{\mathrm{bg}}}\left[i E^{*}(t) P(t)+\text { c.c. }\right],
$$


where we have defined the geometric confinement factor [HUA96]

$$
\Gamma=\frac{\int \mathrm{d}^{3} r\left|u_{E}(\boldsymbol{r})\right|^{2} u_{P}(\boldsymbol{r})}{\int \mathrm{d}^{3} r\left|u_{E}(\boldsymbol{r})\right|^{2}} .
$$

This definition allows one to move the complete spatial dependence of Eq. (2.33) into a single variable $\Gamma$. The geometric confinement factor can be understood as the overlap of the active medium with the optical mode, as illustrated in Fig. 2.4. The thus simplified electric field dynamics can be written as

$$
\frac{\partial}{\partial t} E(t)=\frac{i \omega \Gamma}{2 \varepsilon_{0} \varepsilon_{\mathrm{bg}}} P(t)
$$

Note that Eq. (2.41) does not satisfy Eq. (2.33) at every point in space, but instead only fulfills its space-integrated form. Therefore, only the dynamic evolution of the space-integrated mode amplitude can be described. Changes in the spatial mode profile could in principle occur due to changes of the optical properties of the active medium during operation, e.g., by gain or index guiding effects [SAL92, DRU01], which could be implemented as a variation of $\Gamma$ with the operational parameters.

\section{Optical Losses}

The electric field inside the optical cavity is subject to losses. Internal optical losses lead to a decay with rate $\alpha_{i n t}$, which includes effects such as absorption of photons by free carriers in the surrounding semiconductor material and waveguide losses through the sidewalls. As there exist no perfect mirrors, only a part of the electric field can be reflected back into the cavity, while the rest is transmitted through the cavity mirrors. After each cavity round-trip the electric field is therefore reduced by a factor $r_{1} r_{2}$, with $r_{1}, r_{2}$ denoting the mirror reflectivities at either end of the cavity. Integrating over one cavity round-trip time $\tau_{\text {cav }}=n_{\text {bg }} \ell / c_{0}$, with the cavity length $\ell$, yields for the polarization-free cavity

$$
E\left(t+\tau_{\text {cav }}\right)=E(t) r_{1} r_{2} \exp \left(-\alpha_{\text {int }} \tau_{\text {cav }}\right) .
$$

Assuming that $\tau_{\text {cav }}$ is small compared to all other processes determining the electric field dynamics, the above equation can be transformed to a quasi-continuous change in time:

$$
\left.\frac{\partial}{\partial t} E(t)\right|_{\text {losses }}=-\alpha_{\text {int }} E(t)-\frac{1}{\tau_{\text {cav }}} \log \left(r_{1} r_{2}\right) E(t) \equiv-\kappa E(t)
$$


where the losses have been combined to a total loss rate $\kappa$. Inserting these additional losses into Eq. (2.41), we arrive at the dynamic equation governing the time evolution of the electric field inside the cavity:

$$
\frac{\partial}{\partial t} E(t)=\frac{i \omega \Gamma}{2 \varepsilon_{0} \varepsilon_{\mathrm{bg}}} P(t)-\kappa E(t) .
$$

\subsubsection{Maxwell-Bloch Equations}

So far we have described only the dynamics of the electric field in the cavity, but still missing is the interaction with the active medium. As derived in the previous section, the electric field is driven by the active medium polarization. We will therefore derive expressions for the dynamics of the polarization within the active region in second quantization.

The interaction of the semiconductor matter with the incident light field is described by the dipole interaction Hamiltonian in the electron-hole picture [SCU97], consisting of the kinetic and carrier-field interaction Hamiltonian:

$$
\begin{aligned}
H & =H_{\mathrm{kin}}+H_{\mathrm{c}-\mathrm{f}} \\
& =\sum_{\substack{c \\
s}} \varepsilon_{c} a_{c, s}^{\dagger} a_{c, s}+\sum_{\substack{v \\
s}} \varepsilon_{v} d_{v, s}^{\dagger} d_{v, s}+\sum_{\substack{c v \\
s}}\left(\hbar \Omega_{c v} a_{c, s}^{\dagger} d_{v, s}^{\dagger}+\text { H.c. }\right),
\end{aligned}
$$

where $a, d$ denote electron and hole annihilator operators, with the summation indices $c$ and $v$ running over all electron and hole states, respectively, with $s$ their spin, and $\varepsilon_{c}, \varepsilon_{v}$ their single-particle energy. The interaction matrix element is defined as

$$
\hbar \Omega_{c v} \equiv\left\langle c\left|-e_{0} \mathcal{E}(\boldsymbol{r}, t) \cdot \boldsymbol{r}\right| v\right\rangle=-e_{0} \int \mathrm{d}^{3} r \phi_{c}^{*}(\boldsymbol{r}) \mathcal{E}(\boldsymbol{r}, t) \cdot \boldsymbol{r} \phi_{v}(\boldsymbol{r}) .
$$

Assuming that the lateral dimensions of the wave functions of the interacting states are small compared to the electric field wavelength, the electric field can be split off from the matrix element [SCU97], and we can write the carrier-field interaction Hamiltonian in dipole approximation:

$$
H=\sum_{\substack{c \\ s}} \varepsilon_{c} a_{c, s}^{\dagger} a_{c, s}+\sum_{\substack{v \\ s}} \varepsilon_{v} d_{v, s}^{\dagger} d_{v, s}+\sum_{\substack{c v \\ s}}\left(\mu_{c v} a_{c, s}^{\dagger} d_{v, s}^{\dagger}+\text { H.c. }\right) \mathcal{E}\left(\boldsymbol{r}_{c v}, t\right),
$$

with $\mathcal{E}=\mathcal{E} \hat{\boldsymbol{e}}$. The electric field is taken at the coordinate $\boldsymbol{r}_{c v}$, assumed to be the center of mass of the states $c, v$. The dipole interaction transition moment is defined as

$$
\mu_{c v} \equiv\left\langle c\left|-e_{0} \boldsymbol{r} \cdot \hat{\boldsymbol{e}}\right| v\right\rangle
$$


We use Eq. (2.47) to calculate the dynamics of the occupation probabilities $\rho_{e, c, s} \equiv\left\langle a_{c, s}^{\dagger} a_{c, s}\right\rangle, \rho_{h, v, s} \equiv\left\langle d_{c, s}^{\dagger} d_{c, s}\right\rangle$, and the inter-band microscopic polarization $\tilde{p}_{c v, s} \equiv\left\langle d_{h, v, s} a_{e, c, s}\right\rangle$, by applying Heisenberg's equation of motion. The resulting equations of motion are given by

$$
\begin{aligned}
\left.\frac{\partial}{\partial t} \tilde{p}_{c v, s}(t)\right|_{\mathrm{c}-\mathrm{f}} & =-i \omega_{c v} \tilde{p}_{c v, s}(t)-i \frac{\mu_{c v} \mathcal{E}\left(\boldsymbol{r}_{c v}, t\right)}{\hbar}\left(\rho_{e, c, s}(t)+\rho_{h, v, s}(t)-1\right), \\
\left.\frac{\partial}{\partial t} \rho_{e, c, s}(t)\right|_{\mathrm{c}-\mathrm{f}} & =\frac{1}{\hbar} \sum_{v} 2 \operatorname{Im}\left(\tilde{p}_{c v, s}^{*}(t) \mu_{c v} \mathcal{E}\left(\boldsymbol{r}_{c v}, t\right)\right), \\
\left.\frac{\partial}{\partial t} \rho_{h, v, s}(t)\right|_{\mathrm{c}-\mathrm{f}} & =\frac{1}{\hbar} \sum_{c} 2 \operatorname{Im}\left(\tilde{p}_{c v, s}^{*}(t) \mu_{c v} \mathcal{E}\left(\boldsymbol{r}_{c v}, t\right)\right),
\end{aligned}
$$

where the inter-band transition frequency is defined by $\omega_{c v} \equiv \frac{1}{\hbar}\left(\varepsilon_{c}-\varepsilon_{v}\right)$. Inserting the definition of the real electric field $\mathcal{E}=\frac{1}{2}\left(E(\boldsymbol{r}, t) e^{i(\boldsymbol{k} \cdot \boldsymbol{r}-\omega t)}+\right.$ c.c. $)$ into Eq. (2.49), fast oscillating terms $\propto \exp ( \pm i \omega t)$ with the carrier frequency $\omega$ enter the equations. Let us assume $\omega \approx \omega_{c v}$, i.e., the active medium is excited with light close to the inter-band transition energy. We then move into a co-rotating frame by defining

$$
\tilde{p}_{c v, s}(t) \equiv p_{c v, s}(t) e^{i\left(\boldsymbol{k r}_{c v}-\omega t\right)} u_{E}\left(\boldsymbol{r}_{c v}\right)
$$

leading to

$$
\begin{aligned}
&\left.\frac{\partial}{\partial t} p_{c v, s}(t)\right|_{\mathrm{c}-\mathrm{f}}=-i\left(\omega_{c v}-\omega\right) p_{c v, s}(t)-i \frac{\mu_{c v}}{2 \hbar}(E(t)\left.+E^{*}(t) e^{-2 i\left(\boldsymbol{k} \boldsymbol{r}_{c v}-\omega t+\phi\left(\boldsymbol{r}_{c v}\right)\right)}\right) \times \\
& \times\left(\rho_{e, c, s}(t)+\rho_{h, v, s}(t)-1\right),
\end{aligned}
$$

with $\phi(\boldsymbol{r}) \equiv \arg \left(u_{E}(\boldsymbol{r})\right)$. As $\omega$ for infra-red frequencies is in the order of $\approx \mathrm{fs}^{-1}$, which is much faster than the active medium dynamics, the fast oscillating term $\propto E^{*}(t) e^{2 i \omega t}$ can be assumed to average out and is consequently left out. This approximation is the rotating wave approximation, i.e., $p_{c v, s}$ is assumed to couple only to frequencies close to the inter-band frequency. Furthermore, we assume that only direct inter-band transitions contribute considerably to the light-matter interaction, i.e., any given state $c$ couples only to one specific state $v$ and vice versa. In the quantum-well this corresponds to taking into account only optical transitions without momentum transfer, thus only $p_{\boldsymbol{k} k}$ remain. In the quantumdots, only transitions $p_{G S}, p_{E S}$ between the electron and hole ground states or excited states, respectively, are kept. The spin index $s$ is from here on suppressed 
for notational simplicity. Thus, for the slowly varying transition amplitudes $p$ we reach the following coupled equations:

$$
\begin{aligned}
\left.\frac{\partial}{\partial t} p_{c v}(t)\right|_{c-f} & =-i\left(\omega_{c v}-\omega\right) p_{c v}(t)-i \frac{\mu_{c v} E(t)}{2 \hbar}\left(\rho_{e, c}(t)+\rho_{h, v}(t)-1\right), \\
\left.\frac{\partial}{\partial t} \rho_{e, c}(t)\right|_{c-f} & =\frac{1}{\hbar} \operatorname{Im}\left(p_{c v}^{*}(t) \mu_{c v} E(t)\left|u_{E}\left(\boldsymbol{r}_{c v}\right)\right|^{2}\right) \\
\left.\frac{\partial}{\partial t} \rho_{h, v}(t)\right|_{c-f} & =\frac{1}{\hbar} \operatorname{Im}\left(p_{c v}^{*}(t) \mu_{c v} E(t)\left|u_{E}\left(\boldsymbol{r}_{c v}\right)\right|^{2}\right) .
\end{aligned}
$$

So far, the effects of many-body interactions on the light-matter dynamics has not been taken into account. Similar to the derivations in Section 2.2, by including the carrier-carrier and carrier-phonon interaction Hamiltonians for calculating the time-derivatives, these many-body effects can be taken into account. Two types of modifications to the free-carrier results obtained so far can be classified: First-order effects, i.e., terms linear in the interaction matrix elements, lead to changes in the transition energies and the microscopic polarization amplitudes, known as bandgap renormalization and Coulomb enhancement [HAU89, CHO99, SCH01d, CHO05, LIN10]. Second-order effects lead to scattering between charge carriers, as derived earlier for the occupation probabilities. These scattering events not only redistribute charge carriers, but also lead to a decoherence of the involved states and thus to a decay of the microscopic polarization, known as dephasing [BOR01a, SCH04e, NIL05, LOR06a, VU06, KOP11]. In the following, these many-body processes are not explicitly taken into account, but instead modeled by a single decay time constant, $T_{2}$, characterizing the lifetime of the microscopic polarization. We thus arrive at

$$
\begin{aligned}
\left.\frac{\partial}{\partial t} p_{c v}(t)\right|_{c-\mathrm{f}}= & -\left[i\left(\omega_{c v}-\omega\right)+\frac{1}{T_{2}}\right] p_{c v}(t) \\
& -i \frac{\mu_{c v} E(t)}{2 \hbar}\left(\rho_{e, c}(t)+\rho_{h, v}(t)-1\right) .
\end{aligned}
$$

Assuming now that the electric field amplitude changes only little over the active region volume, we can set $\left|u_{E}(\boldsymbol{r})\right|$ to be constant. By scaling of $E(t)$ and $p_{c v}(t)$ such that $\left|u_{E}(\boldsymbol{r})\right|=1$ within the active region, $E(t)$ describes the actual electric 
field amplitude in the active region, and the Bloch equations can be simplified to a space-independent form:

$$
\begin{aligned}
\left.\frac{\partial}{\partial t} p_{c v}(t)\right|_{\mathrm{c}-\mathrm{f}}= & -\left[i\left(\omega_{c v}-\omega\right)+\frac{1}{T_{2}}\right] p_{c v}(t) \\
& -i \frac{\mu_{c v} E(t)}{2 \hbar}\left(\rho_{e, c}(t)+\rho_{h, v}(t)-1\right), \\
\left.\frac{\partial}{\partial t} \rho_{e, c}(t)\right|_{\mathrm{c}-\mathrm{f}}= & \operatorname{Im}\left(p_{c v}^{*}(t) \mu_{c v} E(t)\right), \\
\left.\frac{\partial}{\partial t} \rho_{h, v}(t)\right|_{\mathrm{c}-\mathrm{f}}= & \operatorname{Im}\left(p_{c v}^{*}(t) \mu_{c v} E(t)\right) .
\end{aligned}
$$

Defining the macroscopic polarization amplitude as the dipole density,

$$
P(t)=\frac{2}{V_{\text {act }}} \sum_{\substack{c \\ s}} \mu_{c v}^{*} p_{c v}(t)
$$

where $V_{\text {act }} \equiv \int \mathrm{d}^{3} r u_{P}(\boldsymbol{r})$ is the active region volume, we are able to write the electric field dynamics as

$$
\frac{\partial}{\partial t} E(t)=\frac{i \omega \Gamma}{2 \varepsilon_{0} \varepsilon_{\mathrm{bg}}} \frac{2}{V_{\mathrm{act}}} \sum_{\substack{c \\ s}} \mu_{c v}^{*} p_{c v}(t)-\kappa E(t) .
$$

This equation together with Eqs. (2.54) then form the Maxwell-Bloch equations, determining the light-matter interaction in the semiconductor device. 


\subsection{Quantum-Dot Laser Rate Equations}

\subsubsection{Maxwell-Bloch Laser Rate Equations}

We now apply the previously derived dynamic equations to the considered quantum-dot optoelectronic laser devices. In order to accurately describe its dynamics, the behavior of the optically interacting quantum-dots as well as the charge carriers in the surrounding quantum-well structure must be taken into account. The InAs/InGaAs quantum-dots considered here are supposed to contain two localized electron and hole levels, the energetically lowest one denoted as the ground state, the higher one as excited state. Simpler models treating the quantum-dot confinement potential as a harmonic oscillator potential lead to a two-fold degenerate excited state due to the rotational symmetry with respect to the growth axis [NIE04]. More realistic models, however, lift this degeneracy [SCH07f]. Nevertheless, the difference in the localization energy between the two first excited states is in the order a few $\mathrm{meV}$, and is thus neglected in the following. Additional to the electronic degeneracy due to the geometric shape of the QDs, all states are assumed to be twofold spin-degenerate. The energy band structure of the considered quantum-dot structure is schematically depicted in Fig. 2.5.

As seen in Sect. 2.2.1, the carrier scattering into the quantum-dot states strongly depends on the occupation of the surrounding carrier reservoir states. Thus, in order to dynamically account for the scattering processes, the charge-carrier dynamics in the reservoir must be taken into account. Following the argumentation of

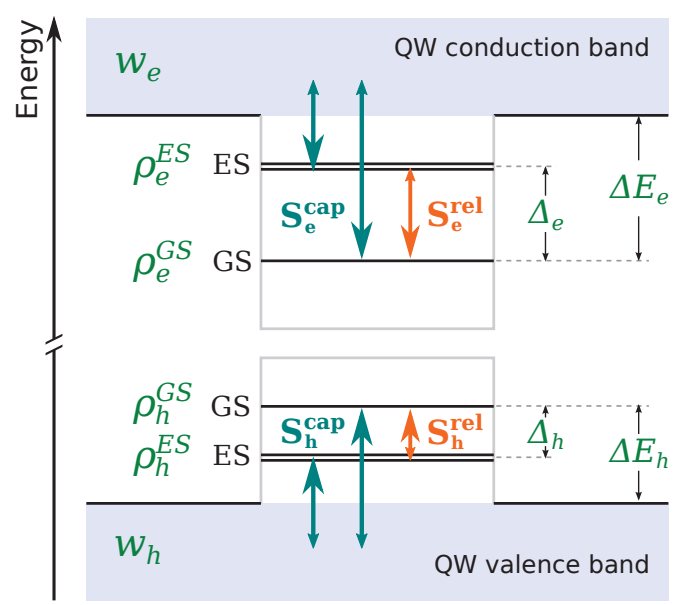

Figure 2.5.: Energy scheme of the considered dot-in-a-well (DWELL) structure. The quantum-dot ground states lie $\Delta E_{b}$ below the quantum-well band edge, with an energy spacing of $\Delta_{b}$ between ground and frist excited state. $S_{b}^{\text {cap }}$ and $S_{b}^{\text {rel }}$ denote direct capture and relaxation scattering processes, respectively. 
rapid equilibration of the charge carriers within the reservoir states, only the total charge-carrier densities $w_{e}, w_{h}$ per unit area in the reservoir must be considered, as the carrier distribution can be calculated from the corresponding quasi-Fermi function.

In the following, let $b \in\{e, h\}$ denote electrons and holes, and $m \in\{$ GS, ES $\}$ quantum-dot ground and excited state, respectively, with $\nu_{m}$ their degree of degeneracy, excluding spin. The dynamic equation of the reservoir carrier density can then in general be written as:

$$
\frac{\mathrm{d}}{\mathrm{d} t} w_{b}=\frac{J}{e_{0}}-r_{\text {loss }}^{w}-\frac{1}{A_{\text {act }}} \sum_{m, i} 2 \nu_{m}\left[S_{b, m, i}^{\text {in,cap }} \rho_{b, m, i}-S_{b, m, i}^{\text {out,cap }}\left(1-\rho_{b, m, i}\right)\right]-\left.\frac{\partial w_{b}}{\partial t}\right|_{\text {stim }},
$$

where $J$ is the electrical pump current density per unit area, $e_{0}$ is the electron charge, $r_{\text {loss }}^{w}$ is a general loss term, and $A_{\text {act }}$ is the area of the active region. The sum runs over all confined quantum-dot states in the $m^{\text {th }}$ quantum-dot excited state, denoted by the index $i$, with the factor 2 accounting for spin degeneracy. The stimulated recombination contribution is given by

$$
\left.\frac{\partial w_{b}}{\partial t}\right|_{\text {stim }}=\frac{2}{A_{\text {act }}} \sum_{\boldsymbol{k}^{2 \mathrm{D}}} \operatorname{Im}\left(p_{\boldsymbol{k}}^{2 \mathrm{D}^{*}}(t) \mu_{\boldsymbol{k}}^{2 \mathrm{D}} E(t)\right),
$$

with the microscopic polarization and dipole moment of the corresponding $\boldsymbol{k}$-state $p_{\boldsymbol{k}}^{2 \mathrm{D}}$ and $\mu_{\boldsymbol{k}}^{2 \mathrm{D}}$, respectively. Under the assumption that the reservoir charge carriers are in quasi-equilibrium, the dynamic equation for the reservoir polarization can be written as

$$
\begin{aligned}
\frac{\mathrm{d}}{\mathrm{d} t} p_{\boldsymbol{k}}^{2 \mathrm{D}}(t)= & -\left[i\left(\omega_{\boldsymbol{k}}^{2 \mathrm{D}}-\omega\right)+\frac{1}{T_{2}}\right] p_{\boldsymbol{k}}^{2 \mathrm{D}}(t) \\
& -i \frac{\mu_{k}^{2 \mathrm{D}} E(t)}{2 \hbar}\left(f\left(\varepsilon_{e, k}^{2 \mathrm{D}}, E_{\mathrm{F}, e}^{\mathrm{eq}}, T^{\mathrm{eq}}\right)+f\left(\varepsilon_{h, k}^{2 \mathrm{D}}, E_{\mathrm{F}, h}^{\mathrm{eq}}, T^{\mathrm{eq}}\right)-1\right)
\end{aligned}
$$

with the transition frequency $\omega_{\boldsymbol{k}}^{2 \mathrm{D}}$. The losses are modeled as

$$
r_{\mathrm{loss}}^{w}=-B^{S} w_{e} w_{h}
$$

with a bimolecular recombination rate $B^{S}$ [SCH87], accounting for recombination processes of an electron and a hole within the charge-carrier reservoir. The bimolecular recombination of charge carriers in the reservoir is assumed to be the dominant process at the charge-carrier densities encountered in laser devices. In order to model the full carrier-density dependent losses, one would need to take linear losses, e.g., Shockley-Read-Hall recombination via trap states [SHO52], as well as Auger-assisted recombination processes [BEA59] into account. 


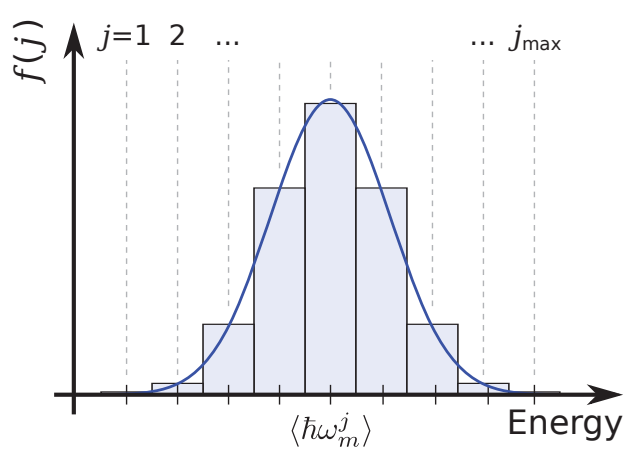

Figure 2.6.: Illustration of the quantum-dot subgroups to model inhomogeneous broadening. The QDs are distributed into $j_{\max }$ subgroups, assumed to follow a Gaussian distribution around the mean transition energy $\left\langle\hbar \omega_{m}^{j}\right\rangle$. The probability mass function $f(j)$ gives the probability for a quantum dot to be found in the $j^{\text {th }}$ subgroup.

The sum over all quantum-dot states $i$ in Eq. (2.57) is equivalent to the sum over all quantum-dots within the active region, which would require keeping track of each individual quantum dot as a dynamic variable. As the number of quantum-dots can easily exceed several million within a typical quantum-dot device, Eq. (2.57) must be reformulated.

Individual quantum-dots within the active layer can differ in shape, size, and material composition. The most apparent effect of these inhomogeneities between different quantum-dots is the broadening of the absorption and emission spectra of quantum-dot optical devices. This inhomogeneous broadening is due to the dependence on the transition energy on the aforementioned quantum-dot parameters. While a single quantum dot exhibits sharp transition energies, the quantum-dot ensemble has a continuous spectrum, which can be accurately modeled by a Gaussian distribution function of the quantum-dot energies around a mean value, with a full-width-at-half-maximum (FWHM) of typically some $10 \mathrm{meV}$ [NAM99].

We now characterize each quantum dot by its transition energy, distributing the quantum-dot ensemble into different subgroups, labeled by the index $j$, with their respective mean transition energy $\omega_{m}^{j}$. We introduce the probability mass function $f(j)$ denoting the ratio of QDs within the $j^{\text {th }}$ subgroup in relation to the total quantum-dot number, as illustrated in Fig. 2.6. Following a Gaussian distribution, $f(j)$ is then given by

$$
f(j)=\frac{1}{\mathcal{N}} \exp \left[-4 \log 2\left(\frac{\hbar \omega_{m}^{j}-\left\langle\hbar \omega_{m}^{j}\right\rangle}{\Delta E_{\text {inh }}}\right)^{2}\right] .
$$


with the normalization constant $\mathcal{N}$ chosen such that $\sum_{j} f(j) \stackrel{!}{=} 1$. For a continuous distribution, one could analytically calculate a closed form for the normalization constant. Due to the discreteness of the subgroups in the simulations, however, $\mathcal{N}$ must be calculated numerically. The inhomogeneous broadening of the optical spectra is given by the sum of the single-particle state broadenings,

$$
\Delta E_{\mathrm{inh}}=\Delta \varepsilon_{e}+\Delta \varepsilon_{h}
$$

where $\Delta \varepsilon_{b}$ is the corresponding electron and hole state broadening. Only the total broadening $\Delta E_{\text {inh }}$ is experimentally readily accessible, e.g., by measurements of the quantum-dot luminescence spectra [LEI08]. For the individual state broadening we assume widths proportional to the localization energy of the given state:

$$
\Delta \varepsilon_{b}=\Delta E_{\text {inh }} \frac{\Delta E_{b}}{\Delta E_{e}+\Delta E_{h}} .
$$

Under the assumption that each quantum dot is fully characterized by its transition energy, i.e., the dynamics of QDs within the same subgroup is identical, the quantum-dot ensemble can be described by a set of dynamic equations for all subgroups. This assumption is in general only approximately fulfilled, as the energy of the confined quantum-dot states depends on a variety of parameters, such that QDs with equal transition energy do not necessarily have to be identical, and their dynamics could thus differ. By averaging the quantum-dot parameters over the whole ensemble the resulting error should nevertheless be small.

For each quantum-dot subgroup a set of optical Bloch equations can be formulated:

$$
\begin{aligned}
\frac{\mathrm{d}}{\mathrm{d} t} p_{m}^{j}(t) & =-\left[i\left(\omega_{m}^{j}-\omega\right)+\frac{1}{T_{2}}\right] p_{m}^{j}(t)-i \frac{\mu_{m} E(t)}{2 \hbar}\left(\rho_{e, m}^{j}(t)+\rho_{h, m}^{j}(t)-1\right), \\
\frac{\mathrm{d}}{\mathrm{d} t} \rho_{b, m}^{j}(t) & =\frac{1}{\hbar} \operatorname{Im}\left(p_{m}^{j}{ }^{*}(t) \mu_{m} E(t)\right)-W_{m} \rho_{e, m}^{j}(t) \rho_{h, m}^{j}(t)+\left.\frac{\partial}{\partial t} \rho_{b, m}^{j}(t)\right|_{\mathrm{sc}} \cdot
\end{aligned}
$$

The dynamic variables $p_{m}^{j}(t), \rho_{m, b}^{j}(t)$ then describe the inter-band polarization and occupation probabilities of electron and holes in the $m^{\text {th }}$ quantum-dot state of the $j^{\text {th }}$ subgroup, respectively. In addition to the Bloch equations derived in Eqs. (2.54), an additional term accounting for the spontaneous recombination of 
quantum-dot charge carriers has been introduced, with the recombination rate $W_{m}$. The charge-carrier scattering contribution was derived in Eqs. (2.15):

$$
\begin{aligned}
\left.\frac{\partial}{\partial t} \rho_{b, \mathrm{GS}}^{j}(t)\right|_{\mathrm{sc}} & =S_{b, \mathrm{GS}}^{j, \mathrm{cap}}(t)+S_{b}^{j, \mathrm{rel}}(t), \\
\left.\frac{\partial}{\partial t} \rho_{b, \mathrm{ES}}^{j}(t)\right|_{\mathrm{sc}} & =S_{b, \mathrm{ES}}^{j, \mathrm{cap}}(t)-\frac{1}{2} S_{b}^{j, \mathrm{rel}}(t),
\end{aligned}
$$

with the individual contributions

$$
\begin{aligned}
S_{b, m}^{j, \mathrm{cap}}(t)= & S_{b, m}^{\mathrm{cap}, \mathrm{in}}\left(w_{e}, w_{h}, T^{\mathrm{eq}}\right)\left[1-\rho_{b, m}^{j}(t)\right] \\
& -S_{b, m}^{\mathrm{cap}, \text { out }}\left(w_{e}, w_{h}, T^{\mathrm{eq}}\right) \rho_{b, m}^{j}(t) \\
S_{b}^{j, \text { rel }}(t)= & S_{b}^{\mathrm{rel}, \mathrm{in}}\left(w_{e}, w_{h}, T^{\mathrm{eq}}\right)\left[1-\rho_{b, \mathrm{GS}}^{j}(t)\right] \rho_{b, \mathrm{ES}}^{j}(t) \\
& -S_{b}^{\mathrm{rel}, \text { out }}\left(w_{e}, w_{h}, T^{\mathrm{eq}}\right) \rho_{b, \mathrm{GS}}^{j}(t)\left[1-\rho_{b, \mathrm{ES}}^{j}(t)\right] .
\end{aligned}
$$

Here, $S_{b, m}^{\mathrm{cap}, \text { in }}$ and $S_{b}^{\mathrm{rel}, \text { in }}$ describe the quantum-dot in-scattering rates by direct capture from the quantum well and by intra-dot relaxation from the ES to GS, respectively, which depend nonlinearly on the carrier reservoir charge-carrier densities and the quasi-equilibrium carrier temperature. The corresponding out-scattering rates are calculated via detailed balance relations, Eqs. (2.22).

We consider only relaxation between quantum-dot states between the ground and excited states of the same quantum-dot subgroup, i.e., we assume a direct mapping of one specific excited state energy to a given ground-state energy. This assumption is strictly valid only if the inhomogeneous broadening of the quantumdot states is due to the non-uniformity of one specific quantum-dot parameter. As different quantum-dots might differ, e.g., both in size and shape, it might be possible that any two given quantum-dots share the same ground-state energy but have different excited-state energies, or vice versa. There is an ongoing discussion in the literature whether the assumed one-to-one mapping of ground-state to excitedstate energies is valid [KAP14b, KAP14c], with experimental results supporting the approximate validity of this assumption. We will therefore only consider the chargecarrier relaxation between equal quantum-dot subgroups.

The dynamic equation for the reservoir charge-carrier densities, Eq. (2.57), can be written as:

$$
\frac{\mathrm{d}}{\mathrm{d} t} w_{b}(t)=\frac{J}{e_{0}}-r_{\text {loss }}^{w}(t)-2 N^{\mathrm{QD}} \sum_{j, m} \nu_{m} f(j) S_{b, m}^{j, \mathrm{cap}}(t)-\left.\frac{\partial w_{b}}{\partial t}\right|_{\text {stim }},
$$

where now the sum over all quantum-dot states was replaced by the sum over all quantum-dot subgroups. The sheet density of quantum-dots within the active region per quantum-well layer is given by $N^{\mathrm{QD}}$. 
Using Eqs. (2.55) and (2.56), the dynamic equation for the electric field amplitude of the lasing mode can be written as

$$
\frac{\partial}{\partial t} E(t)=\frac{i \omega \Gamma}{\varepsilon_{0} \varepsilon_{\mathrm{bg}} h^{\mathrm{QW}}}\left[2 N^{\mathrm{QD}} \sum_{j, m} \nu_{m} f(j) \mu_{m}^{*} p_{m}^{j}+\frac{2}{A_{\mathrm{act}}} \sum_{\boldsymbol{k}^{2 \mathrm{D}}} \mu_{k}^{2 \mathrm{D}^{*}} p_{k}^{2 \mathrm{D}}\right]-\kappa E,
$$

where we used $V_{\text {act }}=a_{L} A h^{\mathrm{QW}}$, with $h^{\mathrm{QW}}$ the height of a single quantum-well layer and $a_{L}$ the number of quantum-well layers.

\subsubsection{Adiabatically Eliminated Polarization}

As a further simplification of the previously derived quantum-dot laser model, the dynamics of the microscopic inter-band polarization can be eliminated. The reasoning behind this is the fast dephasing time $T_{2}$ of the polarization, which is usually in the order of $\approx 100 \mathrm{fs}$ at room temperature [BOR01a, BOR02, VU06]. Considering that the charge-carrier scattering times are commonly in the order of a few ps [NIE04, MAJ10, WIL12b, STE13], and the photon lifetime in conventional Fabry-Perot or DFB-type cavities is several ps, this assumption is in many cases justified. All other dynamic variables can then be assumed to be slowly varying, such that the microscopic polarization amplitudes follow a quasi-static relation given by

$$
\frac{\mathrm{d}}{\mathrm{d} t} p_{m}^{j}(t)=0
$$

Inserting the dynamic equation for the polarization Eq. (2.64a) then yields

$$
p_{m}^{j}(t)=-i T_{2} \frac{\mu_{m} E(t)}{2 \hbar}\left(\rho_{e, m}^{j}(t)+\rho_{h, m}^{j}(t)-1\right)\left(\frac{1-i T_{2}\left(\omega_{m}^{j}-\omega\right)}{1+\left[T_{2}\left(\omega_{m}^{j}-\omega\right)\right]^{2}}\right),
$$

with analogous expressions for the reservoir optical transitions. Inserting this expression in the charge carrier and electric field dynamic equations yields

$$
\begin{aligned}
\frac{\partial}{\partial t} \rho_{b, m}^{j}(t)= & -\operatorname{Re}\left(g_{m}^{j}\right)\left(\rho_{e, m}^{j}(t)+\rho_{h, m}^{j}(t)-1\right)|E|^{2} \\
& -W_{m} \rho_{e, m}^{j}(t) \rho_{h, m}^{j}(t)+\left.\frac{\partial}{\partial t} \rho_{b, m}^{j}(t)\right|_{\mathrm{sc}}, \\
\frac{\partial}{\partial t} E(t)= & g(t) E(t)-\kappa E(t),
\end{aligned}
$$

with the complex gain coefficient of each subgroup

$$
g_{m}^{j}=\frac{T_{2}\left|\mu_{m}\right|^{2}}{2 \hbar^{2}}\left(\frac{1-i T_{2}\left(\omega_{m}^{j}-\omega\right)}{1+\left[T_{2}\left(\omega_{m}^{j}-\omega\right)\right]^{2}}\right) .
$$




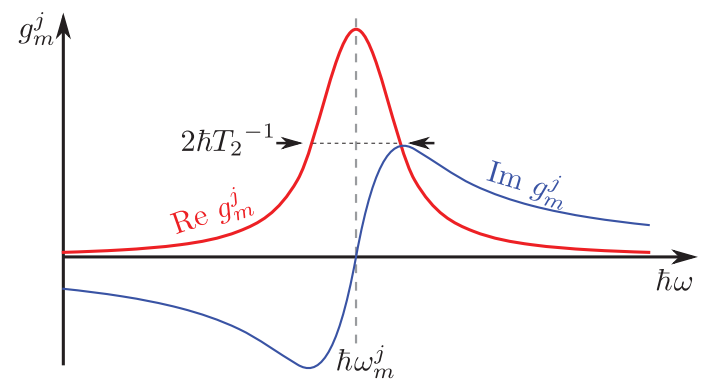

Figure 2.7.: Lorentzian gain profile of a single optical transition. The real part of the quantum-dot gain coefficient $g_{m}^{j}$ has a Lorentzian profile around the carrier frequency $\omega$, the imaginary part becomes extremal at $\left|\omega-\omega_{m}^{j}\right|=T_{2}^{-1}$, while vanishing for resonant transitionts $\omega=\omega_{m}^{j}$. Note the slower decay of the imaginary part compared to the real part for optical frequencies far from the transition frequency.

The gain coefficients $g_{k}^{2 \mathrm{D}}$ for the quantum-well inter-band transitions can be written in the same form. Equation (2.73) leads to a Lorentzian-shaped gain spectrum for each individual optical transition, with a FWHM of $2 \hbar T_{2}{ }^{-1}$. The corresponding imaginary part of the gain vanishes directly at the transition but becomes large at $\hbar \omega \pm \hbar T_{2}{ }^{-1}$ and then decays slowly towards higher detunings from the carrier frequency $\omega$, as illustrated in Fig. 2.7. This means that for large detuning of the optical field frequency from a given optical transition, the imaginary part of the gain coefficient will predominate. Thus, if we assume the lasing frequency is detuned far enough from the reservoir transitions, we can neglect the real part of the corresponding reservoir gain coefficients. This allows us to neglect the stimulated recombination contribution in the dynamic equation for the reservoir charge-carrier densities:

$$
\left.\operatorname{Re} g_{k}^{2 \mathrm{D}} \approx 0 \quad \Rightarrow \quad \frac{\partial w_{b}}{\partial t}\right|_{\text {stim }} \approx 0 .
$$

The resulting complex gain can then be written as

$$
\begin{aligned}
g(t)= & \frac{\hbar \omega \Gamma}{\varepsilon_{0} \varepsilon_{\mathrm{bg}} h \mathrm{QW}}\left[2 N^{\mathrm{QD}} \sum_{j, m} \nu_{m} f(j) g_{m}^{j}\left(\rho_{e, m}^{j}(t)+\rho_{h, m}^{j}(t)-1\right)\right. \\
& \left.+\frac{2 i}{A_{\mathrm{act}}} \sum_{\boldsymbol{k}^{2 \mathrm{D}}} \operatorname{Im} g_{\boldsymbol{k}}^{2 \mathrm{D}}\left(f\left(\varepsilon_{e, \boldsymbol{k}}^{2 \mathrm{D}}, E_{\mathrm{F}, e}^{\mathrm{eq}}, T^{\mathrm{eq}}\right)+f\left(\varepsilon_{h, k}^{2 \mathrm{D}}, E_{\mathrm{F}, h}^{\mathrm{eq}}, T^{\mathrm{eq}}\right)-1\right)\right] .
\end{aligned}
$$

The downside of using the adiabatically eliminated polarization is the loss of optical frequency dependence. By assuming $\frac{\partial}{\partial t} p_{m}^{j}(t)=0$, the response of the active medium is fixed to a single frequency $\omega$, since $p_{m}^{j}$ is the polarization within the rotating frame of the carrier frequency $\omega$. Taking the full polarization dynamics into 
account, an electric field $E(t) \propto \exp (-i \Delta \omega t)$ would excite optical transitions at any arbitrary frequency $(\omega+\Delta \omega)$. However, the adiabatically eliminated polarization reacts to every electric field as if it was centered at the frequency $\omega$. Thus, when a spectrally broad electric field signal needs to be treated, the full polarization equations must be taken into account. For narrow signals, as, e.g., in single-mode laser devices, the adiabatically eliminated polarization will yield satisfyingly accurate results, with the advantage of lower complexity of the differential equation system.

\subsubsection{Modeling of Spontaneous Emission}

So far we have only considered the stimulated emission contributing to the electric field in the laser cavity, but have neglected spontaneous emission. In a rigorous derivation, spontaneous emission can only be derived from a fully quantummechanical description of the electric field [HAK83a, LOU00], which goes beyond the semi-classical picture used in this work. Instead, we will derive the spontaneous emission terms phenomenologically from energy conservation criteria.

We have written the spontaneous charge-carrier losses in the quantum-dot states as

$$
\left.\frac{\partial}{\partial t} \rho_{b, m}^{j}(t)\right|_{\mathrm{sp}}=-W_{m} \rho_{e, m}^{j} \rho_{h, m}^{j}
$$

The total energy lost by this recombination process can be calculated to

$$
2 Z^{\mathrm{QD}} \sum_{j, m} \nu_{m} f(j) W_{m} \rho_{e, m}^{j} \rho_{h, m}^{j} \hbar \omega_{m}^{j},
$$

with the total quantum-dot number $Z^{\mathrm{QD}}$. We now assume that only a fraction $\beta$ of the spontaneously recombining charge-carriers emit a photon into the lasing mode. The remaining recombination processes are assumed to either be non-radiative, or emit photons into other modes. The change in the optical energy density $\left\langle u_{\mathrm{opt}}\right\rangle$ in the laser mode is thus given by

$$
\left.\frac{\partial}{\partial t}\left\langle u_{\mathrm{opt}}\right\rangle\right|_{\mathrm{sp}}=\beta \frac{\Gamma 2 N^{\mathrm{QD}}}{h^{\mathrm{QW}}} \sum_{j, m} \nu_{m} f(j) W_{m} \rho_{e, m}^{j} \rho_{h, m}^{j} \hbar \omega_{m}^{j} .
$$

From the electric field amplitude, the optical energy density can furthermore be calculated to

$$
\left\langle u_{\mathrm{opt}}\right\rangle=\frac{\varepsilon_{\mathrm{bg}} \varepsilon_{0}}{2}|E|^{2} .
$$


As the spontaneous emission process can be viewed as a stochastic process with random phase, the contribution to the electric field can be written in terms of a Gaussian white noise process:

$$
\left.\frac{\partial}{\partial t} E(t)\right|_{\mathrm{sp}}=\sqrt{\frac{D_{\mathrm{sp}}}{2}} \xi(t)
$$

with the complex white noise term $\xi(t)=\xi^{\prime}(t)+i \xi^{\prime \prime}(t)$, with $\xi^{\prime}, \xi^{\prime \prime} \in \mathbb{R}$, and the spontaneous emission amplitude $D_{\mathrm{sp}}$. The individual terms $\xi^{\prime}, \xi^{\prime \prime}$ are assumed to be uncorrelated Gaussian white noise processes. From this the average change in $|E|^{2}$ can be shown to be [GAR06, FLU07]:

$$
\left.\frac{\partial}{\partial t}\left\langle|E(t)|^{2}\right\rangle\right|_{\mathrm{sp}}=D_{\mathrm{sp}}
$$

Comparing Eqs. (2.78) and (2.81), the spontaneous emission strength is calculated to

$$
D_{\mathrm{sp}}=2 \beta \frac{\Gamma 2 N^{\mathrm{QD}}}{\varepsilon_{\mathrm{bg}} \varepsilon_{0} h^{\mathrm{QW}}} \sum_{j, m} \nu_{m} f(j) W_{m} \rho_{e, m}^{j} \rho_{h, m}^{j} \hbar \omega_{m}^{j} .
$$

\section{Deterministic Approximation}

The time-evolution of $|E|^{2}$ can also be written as

$$
\frac{\partial}{\partial t}|E(t)|^{2}=2|E| \frac{\partial}{\partial t}|E|,
$$

which leads to the deterministic approximation of spontaneous emission:

$$
\left.\frac{\partial}{\partial t} E(t)\right|_{\mathrm{sp}}=\frac{D_{\mathrm{sp}}}{2 E^{*}}
$$

While numerically easier to implement than the stochastic approach, this approximation, however, diverges for $E=0$. A description in the photon picture could circumvent this problem.

\subsubsection{Carrier-Induced Gain and Refractive Index Changes}

From Eq. (2.72) it becomes clear that the real part of the gain coefficients $g_{m}^{j}$ contribute to the amplitude gain or loss of the electric field. The imaginary parts lead to a rotation of $E(t)$ in the complex plane. Since $E(t)$ is the slowly varying amplitude of the electric field in a rotating frame with the carrier frequency $\omega$, the 
imaginary part of the gain leads to a frequency shift of the electric field towards $\omega-\operatorname{Im} g(t)$.

This shift of the electric field frequency induced by interaction with the active medium can be understood as a change in the optical length of the cavity due to a change in refractive index. Writing the total medium susceptibility as

$$
\chi(t)=\chi_{\mathrm{bg}}+\delta \chi(t)
$$

with the charge-carrier-induced susceptibility $\delta \chi$, which is related to the electric field gain via

$$
\delta \chi(t)=\frac{2 \varepsilon_{\mathrm{bg}}}{i \omega} g(t)
$$

results in a refractive index

$$
\begin{aligned}
n(t) & =\sqrt{1+\chi(t)} \\
& =\sqrt{\varepsilon_{\mathrm{bg}}+\frac{2 \varepsilon_{\mathrm{bg}}}{i \omega} g(t)} \\
& =n_{\mathrm{bg}} \sqrt{1+\frac{2}{i \omega} g(t)} \\
& \approx n_{\mathrm{bg}}\left(1+\frac{1}{\omega} \operatorname{Im} g(t)\right),
\end{aligned}
$$

where in the last line we assumed $|g(t)| \ll \omega$. Considering now a Fabry-Perot cavity of length $\ell$, the shifted resonance frequency $\omega_{C}$ of the cavity can be calculated from the resonance condition

$$
\begin{aligned}
\omega_{C}(t) & =q \frac{c_{0}}{2 \pi \ell n(t)} \\
& \approx q \frac{c_{0}}{2 \pi \ell n_{\mathrm{bg}}}\left(1-\frac{1}{\omega} \operatorname{Im} g(t)\right) \\
& =\omega-\operatorname{Im} g(t),
\end{aligned}
$$

with $q \in \mathbb{N}$, and $\omega=q c_{0} /\left(2 \pi \ell n_{\mathrm{bg}}\right)$. The above shows that the frequency shift of the electric field is due to the shift of the cavity resonance frequency. This argumentation is valid under the assumption of slowly varying $g(t)$ in relation to the cavity round-trip time, i.e., that the build-up of the standing electric field can follow the induced refractive index changes adiabatically.

Equations (2.88) and (2.73) reveal that optical transitions with a higher energy than the considered carrier frequency will lead to a negative non-zero contribution to the imaginary part of the gain. Following Eq. (2.88), these transitions will therefore lead to a blue-shift of the resonance frequency with increasing population by 
charge carriers. Correspondingly, transitions at lower frequencies than the optical field will lead to a red-shift. By taking into account only the imaginary part of the gain coefficients of the reservoir states in Eq. (2.75), we neglect the optical gain induced by these transitions, but still take into account the induced refractive index changes.

The direct change of the refractive index by charge carriers in the states of the off-resonant transitions is not the only mechanism by which the optical length and thus the resonance frequency of the laser resonator can change. The charge carriers that are lost in non-radiative recombination processes lead to a heating of the semiconductor lattice by emission of phonons in addition to Joule-heating by the applied current. Due to the expansion of the lattice by the increasing temperature the physical length of the resonator increases, leading to a decrease of the resonance frequency and thus a red-shift of the lasing wavelength. This temperature-induced frequency shift is pronounced, e.g, in edge-emitting laser structures, where the active medium runs across the whole length of the laser cavity. The small physical footprint of vertical-cavity surface-emitting lasers (VCSELs) leads to a reduced heat transfer to the surrounding medium or an attached heat sink and subsequently to a pronounced heating of the semiconductor material and a red-shift [LIU05a].

Furthermore, not all optical transitions have been accounted for in the present model. In addition to the direct optical transitions already considered, also transitions between confined quantum-dot states and the continuum states of the reservoir involving only conduction band or valence band states are possible. The effect of such transitions on the refractive index can be approximately expressed by a modified Drude formula for the optical susceptibility [USK04, LI13b]. In the remainder of this work, we will, however, concentrate on the effects of direct inter-band transitions. 


\subsection{Quantum-Dot Laser Carrier-Heating Model}

Temperature effects in semiconductor optical devices have been previously shown to be important for device design considerations. An increase in lattice and chargecarrier temperature often leads to reduced efficiency and diminished device performance [KLO99, ROS09a]. On the other hand, higher temperatures were shown, e.g., to improve the performance of mode-locked laser devices [CAT07]. Quantum-dot lasers have been theoretically predicted to exhibit lower sensitivity to temperature effects compared to quantum-well devices, due to their confined levels inhibiting thermal escape of optically active carriers to the surrounding reservoir [ARA82]. Experimental findings in some cases confirm an improvement regarding temperature stability, especially in p-doped quantum-dot lasers [OTS04, TAN04, MIK05, SUG05], while other results suggest a sensitivity to temperature in quantum-dot lasers that is comparable to quantum-well devices [KLO99, SHC00]. Taking into account carrier heating and temperature effects can therefore be important for a realistic modeling of quantum-dot optical devices.

In the previous sections, the importance of charge-carrier scattering processes in quantum-dot optical devices has been discussed. The involved Auger processes enter the dynamic equations for the charge-carrier occupations and densities as Boltzmann-like scattering terms. They lead to a redistribution of the charge carriers between the reservoir and quantum-dot states, and, in the process, create so-called hot electrons, which increase the charge carrier distribution temperature. In the following, dynamic equations for the charge-carrier energy and thus the quasiequilibrium temperature will be derived by formulating energy balance equations for the reservoir charge carriers. These equations can then be used to dynamically include carrier heating in quantum-dot optical devices.

\subsubsection{Charge-Carrier Energy and Temperature}

As stressed in the previous sections, Auger processes conserve the total chargecarrier energy by redistributing the charge-carrier population. Specifically, when carriers from reservoir states scatter into the lower quantum-dot confined states, energetically higher reservoir states will be occupied by the Auger-electron or hole. A rigorous way to treat this Auger-heating would involve the description of all $\boldsymbol{k}$-states in the reservoir and resolving every possible scattering process in order to determine the dynamic change of each $\boldsymbol{k}$-state. As was detailed previously, such an approach would be numerically very expensive.

Nevertheless, under the assumption of fast quasi-equilibration of the reservoir carrier distributions, an alternative treatment of the Auger-induced carrier distribution changes can be formulated. In quasi-equilibrium, the carrier distribu- 
tion $\rho_{b, \mathrm{QW}}\left(\varepsilon_{b, k}^{2 \mathrm{D}}\right)$ is given by the quasi-Fermi distribution $f\left(\varepsilon_{b, k}^{2 \mathrm{D}}, E_{\mathrm{F}, b}^{\mathrm{eq}}, T^{\mathrm{eq}}\right)$. In order to fully characterize the carrier distribution, only the quasi-Fermi level and quasi-equilibrium temperature must be known. While the quasi-Fermi level can be inferred from the charge-carrier densities in the carrier reservoir, the quasiequilibrium temperature in most models is assumed to be constant, and thus Auger heating as well as other heating effects are neglected. Here, an energy balance approach is presented that can be used to dynamically calculate the change of the quasi-equilibrium charge-carrier temperature.

Carrier heating has been previously shown to crucially influence the performance of electro-optic devices. For example in quantum-well lasers carrier heating has been shown to contribute to the nonlinear gain compression, i.e., the reduction of differential gain with increasing optical power [WAN97a]. In quantum-well optical amplifiers it was shown that Auger-heating leads to a nonuniform carrier temperature along the device [FEH02]. Carrier heating in quantum-dot lasers has a strong impact on the charge-carrier scattering dynamics due to a change in the detailed balance condition Eq. (2.22) with temperature [USK11], as well as a quantitative change of the Auger-scattering rates [MAJ11].

The total kinetic energy density of the charge carriers in the reservoir states can be written as

$$
u=\sum_{b} \int_{-\infty}^{\infty} \mathrm{d} \varepsilon_{b, k}^{2 \mathrm{D}} \mathcal{D}_{b}\left(\varepsilon_{b, k}^{2 \mathrm{D}}\right) \varepsilon_{b, k}^{2 \mathrm{D}} \rho_{b, \mathrm{QW}}\left(\varepsilon_{b, k}^{2 \mathrm{D}}\right),
$$

which, under the assumption of a quasi-Fermi distribution in the reservoir states, is related to the quasi-equilibrium charge-carrier temperature $T^{\mathrm{eq}}$ via

$$
u^{\mathrm{eq}}=\sum_{b} \int_{-\infty}^{\infty} \mathrm{d} \varepsilon_{b, k}^{2 \mathrm{D}} \mathcal{D}_{b}\left(\varepsilon_{b, k}^{2 \mathrm{D}}\right) \varepsilon_{b, k}^{2 \mathrm{D}}\left[1+\exp \left(\frac{\varepsilon_{b, \boldsymbol{k}}^{2 \mathrm{D}}-E_{\mathrm{F}, b}^{\mathrm{eq}}}{k_{\mathrm{B}} T^{\mathrm{eq}}}\right)\right]^{-1} .
$$

In the case of non-degenerate semiconductors, i.e., for small carrier numbers, the charge carrier distribution in the continuum states approximately follow Boltzmann statistics, and the charge carrier energy density and quasi-equilibrium temperature are simply related via

$$
\begin{aligned}
\left.u^{\mathrm{eq}}\left(w_{e}, w_{h}, T^{\mathrm{eq}}\right)\right|_{\text {Boltzmann }} & \approx \sum_{b} \int_{0}^{\infty} \mathrm{d} \varepsilon_{b, k}^{2 \mathrm{D}} \mathcal{D}_{b}\left(\varepsilon_{b, k}^{2 \mathrm{D}}\right) \varepsilon_{b, k}^{2 \mathrm{D}} \exp \left[\frac{E_{\mathrm{F}, b}^{\mathrm{eq}}-\varepsilon_{b, k}^{2 \mathrm{D}}}{k_{\mathrm{B}} T^{\mathrm{eq}}}\right] \\
& =k_{\mathrm{B}} T^{e q}\left(w_{e}+w_{h}\right)
\end{aligned}
$$

where, for simplicity, the corresponding band edge energies were set to zero. 
Generally, however, semiconductor optoelectronic devices can exceed the lowdensity limit and the above approximation cannot be applied. Instead, the full expression Eq. (2.90) which takes the quasi-Fermi statistics into account must be evaluated.

The quasi-Fermi levels in the reservoir $E_{\mathrm{F}, b}^{\mathrm{eq}}$ can be expressed in terms of the charge-carrier densities $w_{b}$ by using Eq. (2.19). The total charge-carrier energy density then becomes a function of the carrier densities and their quasi-equilibrium temperature: $u^{\mathrm{eq}} \equiv u^{\mathrm{eq}}\left(w_{e}, w_{h}, T^{\mathrm{eq}}\right)$. For constant $w_{e}$ and $w_{h}$, the quasiequilibrium energy density increases monotonically with the temperature. As such, it is possible to invert the function $u^{\mathrm{eq}}\left(w_{e}, w_{h}, T^{\mathrm{eq}}\right)$ and instead calculate the quasiequilibrium carrier temperature from the charge-carrier densities and energy density:

$$
T^{\mathrm{eq}} \equiv T^{\mathrm{eq}}\left(w_{e}, w_{h}, u^{\mathrm{eq}}\right)
$$

In order to calculate the dynamic changes of the charge carrier temperature during operation of the optoelectronic device, dynamic equations for the charge carrier energy must be formulated.

\subsubsection{Carrier Heating by Auger-Scattering Processes}

The change of the total kinetic charge carrier energy due to Auger-scattering processes can be determined by calculating the net energy change of the reservoir charge carriers involved in each considered scattering process. This is illustrated in Fig. 2.8. In a charge carrier capture event, a quantum-well charge carrier at energy $\varepsilon_{1}$ (relative to the quantum-well band edge $E_{b, 0}^{\mathrm{QW}}$ ) fills a vacant quantum-dot state with energy $\varepsilon_{\mathrm{QD}}$, under scattering of a quantum-well carrier from $\varepsilon_{2}$ to the vacant state $\varepsilon_{3}$, where energy conservation dictates $\varepsilon_{3}=\varepsilon_{2}+\left(\varepsilon_{1}-\varepsilon_{\mathrm{QD}}\right)$. The total quantum-well energy change is thus $\Delta U^{\mathrm{QW}}=\varepsilon_{3}-\varepsilon_{2}-\varepsilon_{1}=\varepsilon_{\mathrm{QD}}$, and thus equal to the localization energy of the involved quantum-dot state. Similarly, for intra-dot scattering from the excited to the ground state, a net energy equal to the GS-ES separation $\Delta_{b}$ is added to the total quantum-well charge carrier energy.

Considering now all possible Auger-scattering processes in the considered system, the change of the reservoir energy density due to this scattering can be written as:

$$
\begin{aligned}
\left.\frac{\partial u}{\partial t}\right|_{\text {Auger }}= & \sum_{b} 2 N^{\mathrm{QD}} \sum_{j, m} \nu_{m} f(j)\left(E_{b, 0}^{\mathrm{QW}}-\varepsilon_{b, m}^{\mathrm{QD}, j}\right) S_{b, m}^{j, \mathrm{cap}} \\
& +\sum_{j} \nu_{\mathrm{GS}} f(j)\left(\varepsilon_{b, \mathrm{ES}}^{\mathrm{QD}, j}-\varepsilon_{b, \mathrm{GS}}^{\mathrm{QD}, j}\right) S_{b}^{j, \mathrm{rel}} .
\end{aligned}
$$




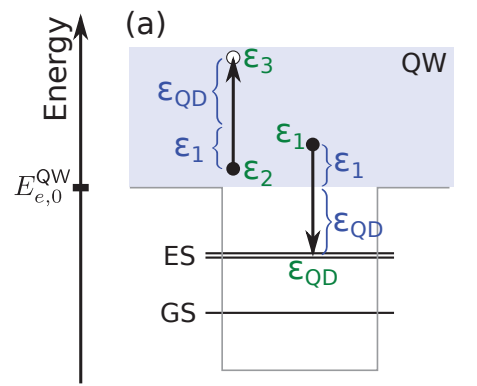

(b)

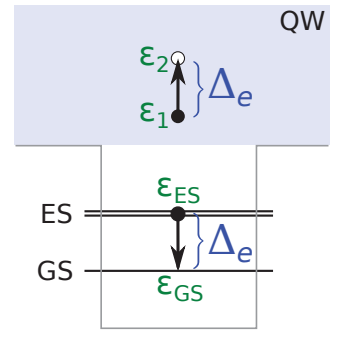

Figure 2.8.: Mechanism of Auger-heating in quantum-dot devices, shown representatively for electrons only. (a) Direct capture processes: a carrier with energy $\varepsilon_{1}$ relative to the quantum-well $(\mathrm{QW})$ band edge $E_{b, 0}^{\mathrm{QW}}$ scatters in to a quantum-dot state $\varepsilon_{\mathrm{QD}}$ below the band edge, while another carrier in the QW gains kinetic energy equal to $\varepsilon_{1}+\varepsilon_{\mathrm{QD}}$. The net energy change in the $\mathrm{QW}$ is then $\varepsilon_{\mathrm{QD}}$. (b) Relaxation process: a carrier relaxes from the quantum-dot excited state (ES) into the ground state (GS). The Auger electron in the QW adds a net energy of $\Delta_{e}$ to the total QW charge-carrier energy.

Here, $S_{b, m}^{j, \text { cap }}$ and $S_{b}^{j, \text { rel }}$ denote the net scattering rates for capture and relaxation processes, respectively, as defined in Eq. (2.66).

\subsubsection{Energy Balance Equations}

In addition to the above-mentioned contribution of Auger-heating, other mechanisms that influence the charge carrier energy need to be be considered. Not only the scattering of charge carriers with quantum-dot states will change the carrier energy density, but also the scattering of carriers into the reservoir by the pumping process. As the charge carriers can be expected to be thermalized with the lattice when they reach the bulk material surrounding the active medium, the energy gained by the capture of a pump carrier into the reservoir is roughly given by the band-gap energy spacing between the reservoir and bulk materials. We thus assume an average pump energy of $\varepsilon_{\text {pump }}$ per charge carrier. The corresponding contribution to the energy density balance is therefore:

$$
\left.\frac{\partial u}{\partial t}\right|_{\text {pump }}=\frac{J}{e_{0}} \varepsilon_{\text {pump }}
$$

Furthermore, the spontaneous recombination of charge carriers in the reservoir will effectively remove energy from it. Here, we assume that the recombination is equally probable for all reservoir carriers, i.e., a recombination event will remove 
twice the average charge carrier energy $\langle u(t)\rangle \equiv u\left(w_{e}+w_{h}\right)^{-1}$ from the reservoir, as both an electron and a hole are simultaneously annihilated in the process:

$$
\left.\frac{\partial u}{\partial t}\right|_{\mathrm{rec}}=\left.\frac{\partial\left(w_{e}+w_{h}\right)}{\partial t}\right|_{\mathrm{rec}}\langle u(t)\rangle=-\frac{2 B^{S} w_{e}(t) w_{h}(t)}{w_{e}(t)+w_{h}(t)} u(t)
$$

Lastly, we have to consider the cooling of the charge carrier gas due to the interaction with lattice phonons. Here, carriers interact with the lattice by emitting a phonon, effectively removing the phonon energy from the total charge carrier energy. This process will cool the carriers towards the lattice temperature $T_{\ell}$. We assume that the rate of cooling is proportional to the excess energy of the charge carrier gas with respect to the thermal energy it would have at $T_{\ell}$ :

$$
\left.\frac{\partial u}{\partial t}\right|_{\text {phon }}=-\gamma_{p}\left[u(t)-u^{\mathrm{eq}}\left(w_{e}, w_{h}, T_{\ell}\right)\right]
$$

The carrier-phonon interaction rate $\gamma_{p}$ is a measure for the timescale on which the carriers are cooled.

Collecting all the above contributions to the energy density balance, we can write the dynamic equation for $u(t)$ as

$$
\frac{\mathrm{d}}{\mathrm{d} t} u(t)=\left.\frac{\partial u}{\partial t}\right|_{\text {pump }}+\left.\frac{\partial u}{\partial t}\right|_{\text {rec }}+\left.\frac{\partial u}{\partial t}\right|_{\text {Auger }}+\left.\frac{\partial u}{\partial t}\right|_{\text {phon }} .
$$




\subsection{Introduction}

The stability of semiconductor laser operation plays a crucial role in almost every possible application of these devices [ERN10b, LUE11b, CHO13a, OTT14]. In most fields of operation, one would require a stable steady-state output with constant intensity that follows any change in external operating parameters instantaneously, thus enabling, e.g., arbitrarily fast switching of the laser output. In reality such requirements can naturally never be met.

The semiconductor laser itself is a nonlinear dynamical system which can exhibit a variety of dynamical instabilities and nonlinearities with respect to parameter changes. In a dynamical sense, the appearance of different solutions and their bifurcations then determine which types of operation are experimentally accessible. In order to understand and correctly predict the laser behavior in different setups, a thorough analysis of the quantum-dot laser system is required. By means of such analysis, one can predict and explain the performance of the laser device under different types of applications as well as determine the stability of different types of solutions. Furthermore, the effects of noise, which is omnipresent in the real world, e.g., due to spontaneous emission of photons or charge carrier injection noise, can be discussed, and the sensitivity of the different solutions to noise can be assessed.

As shown in Chapter 2, the charge carrier dynamics in semiconductor quantumdots strongly depend on the scattering processes between the quantum-dot states and the surrounding charge carrier reservoir. The scattering rates for these processes are in itself highly nonlinear, depending on the reservoir carrier densities and temperature. Compared to conventional bulk and quantum-well semiconductor lasers, where charge carrier equilibration happens on a fast timescale, the carrier dynamics between the quantum-dots and the surrounding material is of the same order as other dynamic timescales. Carrier scattering thus plays an important role in quantum-dot lasers, and its effects become evident in many experimental observations [LUE09, MAJ10]. 
In this chapter the dynamics of quantum-dot lasers will be discussed with focus on the underlying charge-carrier scattering dynamics. The properties and behavior of solitary and directly modulated quantum-dot lasers as well as setups with optical injection or feedback will be discussed. Here, interference effects and thus the phase dynamics of the electric field play an important role. It will be shown that the carrier scattering sensitively influences these phase dynamics by a dynamic shift of the active medium refractive index, which gives rise to important differences in the dynamic response of quantum-dot lasers when compared to conventional lasers.

In Section 3.2, the relaxation oscillations of quantum-dot lasers will be investigated and compared to the predictions of established rate-equation systems. We will determine the dependence of the relaxation oscillation parameters on the charge-carrier scattering rates in quantum-dot lasers and identify different dynamic regimes of laser operation. We then present a minimal rate-equation model for quantum-dot lasers in Section 3.3. This model is used to identify the key aspects of charge-carrier dynamics that determine the unique features of quantum-dot laser dynamics. Analytical expressions for the relaxation oscillation damping and frequency in the limits of slow and fast charge-carrier scattering will be derived.

We will discuss the quantum-dot laser response to direct modulation in Section 3.4, emphasizing the dependence of the small-signal response on the chargecarrier scattering rates. Important differences of the modulation behavior of quantum-dot lasers compared to conventional laser devices will be presented.

In Section 3.5, we will investigate the dynamics of carrier-induced refractive-index changes in quantum-dot lasers. We will discuss the applicability of the commonly used linewidth enhancement factor $\alpha$ in quantum-dot lasers and highlight important effects in the frequency-response of quantum-dot lasers to external perturbations. These lead to important differences to the behavior of conventional laser devices when discussing optical interactions. The quantum-dot laser dynamics under optical injection will thus be discussed in Section 3.6, where we will compare the predictions of our quantum-dot laser model with those using an $\alpha$-factor. In Section 3.7, we will subsequently simplify our laser model and make it suitable for implementation in path-continuation tools, allowing for the investigation of the detailed bifurcation structure of the quantum-dot laser under optical injection. We will extend the investigation to the application of time-delayed optical feedback in Section 3.8 .

Then, we will discuss the frequency chirp and modulation of the quantum-dot laser in response to generalized, frequency-dependent external modulations in Section 3.9, again highlighting the unique features of quantum-dot lasers due to their complex charge-carrier scattering dynamics. Finally, a conclusion will be given in Section 3.10. 


\subsection{Laser Dynamics - Relaxation Oscillations}

In general, the dynamics of lasers is determined from the interaction between the electric field, the inter-band polarization of the active medium, and the charge carrier dynamics. A classification of lasers due to Arecchi et al. [ARE84] characterizes lasers by the order of magnitude of characteristic dynamic timescales of these three parts:

- Class-C lasers are characterized by timescales of the polarization dephasing, charge carrier decay, and cavity photon lifetime on the same order of magnitude. Their dynamics is characterized by the interaction of all three values. Most importantly, solitary class-C lasers can exhibit deterministic chaos after a so-called second laser threshold [OHT06, ZEG88]. This behavior can be explained by the formal identity of the class-C Maxwell-Bloch laser equations to the Lorenz-Haken equation system, which is known to exhibit chaos [WEI88, NIN90]. Examples for class-C lasers include $\mathrm{NH}_{3}, \mathrm{Ne}-\mathrm{Xe}$, and infrared He-Ne lasers.

- In class-B lasers the polarization dephasing lifetime is much smaller than the corresponding photon and carrier lifetimes. The polarization will therefore adiabatically follow the changes in both photon and carrier number. As such, no independent motion of the polarization is possible, reducing the dynamical dimension by one. Class-B lasers include $\mathrm{CO}_{2}$ lasers and most semiconductor lasers.

- Class-A lasers possess photon lifetimes much longer than both the polarization and charge carrier lifetimes. Only the optical intensity remains as a dynamical variable, with both the charge carrier number and polarization adiabatically following its time evolution. With only one dynamic degree of freedom left, class-A lasers cannot exhibit chaos or oscillations. Examples for class-A lasers are dye lasers and visible He-Ne lasers [ОНт06].

\subsubsection{Relaxation Oscillations in Two-Variable Laser Equations}

Semiconductor lasers, including quantum-dot lasers, are generally class-B lasers. As such, they exhibit dynamical behavior that is characterized in most parts by the two-dimensional phase-space given by the optical power (or field amplitude) and the gain, given by the inversion of the optically resonant transitions. Within this effectively two-dimensional phase-plane the laser can exhibit damped oscillatory motion about the fixed point, known as relaxation oscillations (ROs). These oscillations

Parts of this section have been published in [LIN12, LIN14]. 
can be modeled using a simple two-variable rate-equation system [ERN10b, COL12a] for the total charge carrier number $N$ and photon number $S$ :

$$
\begin{aligned}
\frac{\mathrm{d}}{\mathrm{d} t} N & =J-2 g N S-\frac{1}{T_{1}} N \\
\frac{\mathrm{d}}{\mathrm{d} t} S & =2 g N S-2 \kappa S,
\end{aligned}
$$

with a normalized pump current $J$, a gain coefficient $g$, the carrier decay time $T_{1}$, and the photon decay rate $2 \kappa$. The stimulated emission term $2 g N(t) S(t)$ defines the nonlinearity that is the driving source of the laser action and is included in all laser models. An analysis of the fixed points of above equations, i.e. the points which fulfill $\frac{\mathrm{d} N}{\mathrm{~d} t}=\frac{\mathrm{d} S}{\mathrm{~d} t}=0$, readily yields the fixed point of the non-lasing state

$$
N^{*}=\frac{J}{T_{1}} \quad S^{*}=0,
$$

and the lasing state

$$
N^{*}=\frac{\kappa}{g} \quad S^{*}=\frac{1}{2 \kappa}\left(J-J_{\mathrm{th}}\right),
$$

where the threshold pump current $J_{\text {th }}=\frac{\kappa}{g T_{1}}$ was introduced, below which $S^{*} \leq 0$, i.e., the laser is off. The lasing state is characterized by a linearly increasing photon number with $J$, and a constant carrier number $N^{*}=N_{\text {th }}$. The gain is therefore clamped to the threshold gain value, where it first becomes equal to the optical losses. This is a direct consequence of the lasing condition $2 g N \stackrel{!}{=} 2 \kappa$, i.e., the gain exactly cancels out the optical losses in the steady-state.

In order to derive the stability of the above fixed points, the differential equation system will be linearized. In the vicinity of the fixed point solution, i.e., $\delta N \equiv$ $\left(N-N^{*}\right), \delta S \equiv\left(S-S^{*}\right)$ are assumed to be "small", the system can be assumed to follow linear dynamics. We can thus write

$$
\frac{\mathrm{d}}{\mathrm{d} t} \delta N=\left.\frac{\partial}{\partial N}\left[\frac{\mathrm{d} N}{\mathrm{~d} t}\right]\right|_{N^{*}} \delta N+\left.\frac{\partial}{\partial S}\left[\frac{\mathrm{d} N}{\mathrm{~d} t}\right]\right|_{S^{*}} \delta S,
$$

with an analogous expression for $\frac{\mathrm{d}}{\mathrm{d} t} \delta S$. The time evolution can be written using the system's Jacobian matrix:

$$
\frac{\mathrm{d}}{\mathrm{d} t}\left(\begin{array}{c}
\delta N \\
\delta S
\end{array}\right)=\underline{\underline{J}}\left(\begin{array}{l}
\delta N \\
\delta S
\end{array}\right) .
$$


This linearized equation can be solved by using an exponential ansatz. We thus write $\delta N \equiv \widehat{\delta N} e^{\lambda t}$, with some constant $\widehat{\delta N}$. Using the same ansatz for $\delta S$, Eq. (3.5) can be written as

$$
\lambda\left(\begin{array}{l}
\widehat{\delta N} \\
\widehat{\delta S}
\end{array}\right)=\underline{\underline{J}}\left(\begin{array}{l}
\widehat{\delta N} \\
\widehat{\delta S}
\end{array}\right)
$$

The above is then an eigenvalue problem, with $\lambda,(\widehat{\delta N}, \widehat{\delta S})^{T}$ being the eigenvalues and eigenvectors of $\underline{\underline{J}}$, respectively. The eigenvalues allow for a classification of the fixed point as either stable or unstable. For $\lambda>0$, the small deviation from the fixed point will grow exponentially in time, and the system will move away from the fixed point, making it unstable. On the other hand, if $\lambda<0$, the system will asymptotically approach the fixed point - the system is then called asymptotically stable [SCH07]. The so-called Lyapunov-exponents $\lambda$ furthermore allow for the distinction between oscillating and simple decaying solutions. If a pair of complex-conjugate Lyapunov-exponents with non-vanishing imaginary part exist, the system will perform oscillations around the fixed point, with an angular frequency given by the absolute value of the imaginary part.

We now apply the linear stability analysis to the two-variable rate-equation system. The eigenvalues of the Jacobian at the lasing fixed point, Eq. (3.3), can be calculated to

$$
\begin{aligned}
\lambda_{1,2} & =-\frac{1}{2}\left(2 g S^{*}+\frac{1}{T_{1}}\right) \pm i \sqrt{4 \kappa g S^{*}-\frac{1}{4}\left(2 g S^{*}+\frac{1}{T_{1}}\right)^{2}} \\
& \equiv \Gamma_{\mathrm{RO}} \pm i \sqrt{\omega_{\mathrm{res}}^{2}-\Gamma_{\mathrm{RO}}^{2}} .
\end{aligned}
$$

Here, the relaxation-oscillation damping rate $\Gamma_{\mathrm{RO}}$ as well as the relaxation-oscillation resonance frequency $\omega_{\text {res }}$ was introduced. The fact that the real part of the eigenvalues is always negative (or, $\Gamma_{\mathrm{RO}}$ positive) for all $J>J_{\mathrm{th}}$, means that the lasing fixed point is always stable, and slight deviations from it will decay in time with the relaxation-oscillation damping rate. Furthermore, oscillations about the fixed point with the angular frequency $\omega_{\mathrm{RO}} \equiv \sqrt{\omega_{\text {res }}^{2}-\Gamma_{\mathrm{RO}}^{2}}$ will appear, provided $\Gamma_{\mathrm{RO}}<\omega_{\text {res }}$. The fixed point is thus a stable focus. As can be seen, the relaxation oscillation damping $\Gamma_{\mathrm{RO}}$ scales linearly with the steady-state laser intensity, whereas the oscillation frequency scales with its square root. For increasing power, the relaxation oscillations will therefore become less pronounced.

The relaxation oscillations are illustrated in Fig. $3.1 \mathrm{a}$ and $3.1 \mathrm{~b}$, showing the time-series of the two dynamic variables and the trajectory in the $(N, S)$-phasespace, using Eqs. (3.1). Clearly visible are the damped relaxation oscillations in both dynamic variables. When the trajectory is still far from the fixed point, the 

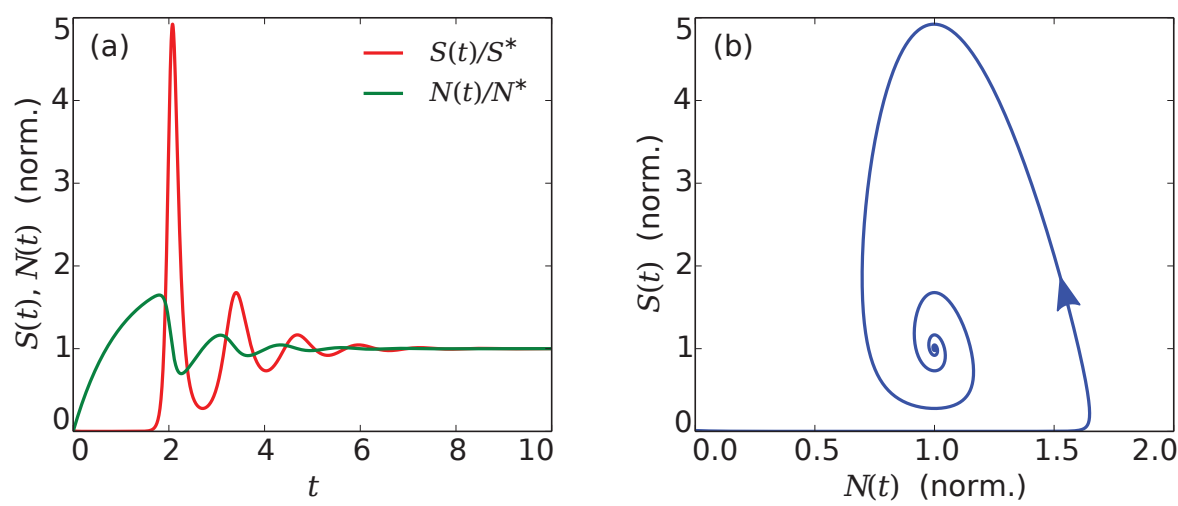

Figure 3.1.: Relaxation oscillations after the laser turn-on in a two-variable laser rateequation system from simulation of Eqs. (3.1) with $T_{1}=1, g=1,2 \kappa=25, J=50=$ $2 J_{\text {th }}$, and $S(0)=0.01, N(0)=0$. (a) Time-series of the normalized optical power $S / S^{*}$ and the normalized charge carrier number $N / N^{*}$. (b) Trajectory in the $(N, S)$-phasespace.

oscillations are highly anharmonic, owing to the nonlinearity in the rate equations, and only become harmonic close to the fixed point.

The relaxation oscillations of a laser define the laser response to small perturbations from its lasing fixed point. Therefore, the relaxation oscillation damping and frequency already can give an indication about the stability properties of lasers, as well as its small-signal modulation capabilities. For example, when the laser operation is perturbed by optical feedback, e.g. due to reflections, the critical feedback strength needed to destabilize the $\mathrm{cw}$ operation of the laser is directly proportional to the relaxation-oscillation damping $\Gamma_{\mathrm{RO}}$ [MOR92, LEV95, ОTT10]. In applications where a constant output power is required, strong damping of the relaxation oscillations is therefore favorable. The relaxation-oscillation frequency is important for modulation applications, as a modulation of the laser with a frequency near $\omega_{\text {RO }}$ can resonantly excite relaxation oscillations and lead to unwanted nonuniform laser responses [CHU95], as will be discussed in Sec. 3.4.

From Eq. (3.7) it can be seen that the charge carrier lifetime $T_{1}$ enters in both the frequency and damping of the relaxation oscillations. In the case of quantumdot lasers, it is in general not possible to assign a single characteristic lifetime to the charge carriers, due to the coupling of quantum-dot and reservoir states. The relaxation oscillation behavior of quantum-dot lasers can therefore be expected to differ from conventional lasers and to depend on the scattering lifetimes as well as the respective charge carrier decay (recombination) times. In the following sections, the dependence of the relaxation oscillation dynamics in quantum-dot lasers and 
their dynamical performance on the underlying scattering processes and charge carrier dynamics will be discussed.

\subsubsection{Turn-On Dynamics of Quantum-Dot Lasers}

We now utilize the quantum-dot laser rate equations derived in Section 2.4 to simulate the turn-on dynamics of quantum-dot lasers. We consider a $1.2 \mathrm{~mm}$ long ridge waveguide edge-emitting dot-in-a-well (DWELL) single-mode laser device, with a number of $a_{L}=15$ stacked InGaAs quantum-wells, each embedding a density of $N^{\mathrm{QD}}$ InAs quantum-dots. We consider an emission wavelength around $\lambda=1.3 \mu \mathrm{m}$ and set the carrier-frequency of our rotating frame to $\omega=230 \mathrm{THz}$. The quantumdots are assumed to have a localized ground-state and a twofold degenerate excited state both for electrons and holes, each with an additional twofold spin-degeneracy.

The equations governing the dynamics of charge carriers in the device are thus given by:

$$
\begin{aligned}
\frac{\mathrm{d}}{\mathrm{d} t} w_{b} & =\frac{J}{e_{0}}-B^{S} w_{e} w_{h}-2 N^{\mathrm{QD}} \sum_{j, m} \nu_{m} f(j) S_{b, m}^{j, \text { cap }} \\
\frac{\mathrm{d}}{\mathrm{d} t} \rho_{b, m}^{j} & =-\operatorname{Re}\left(g_{m}^{j}\right)\left(\rho_{e, m}^{j}+\rho_{h, m}^{j}-1\right)|E|^{2}-W_{m} \rho_{e, m}^{j} \rho_{h, m}^{j}+S_{b, m}^{j \mathrm{cap}} \pm \frac{1}{\nu_{m}} S_{b}^{j, \text { rel }}
\end{aligned}
$$

where the carrier relaxation rate enters with a positive (negative) sign for $m=$ GS $(m=\mathrm{ES})$. The charge-carrier in-scattering rates $S_{b, m}^{\mathrm{cap} / \mathrm{rel}, \mathrm{in}}\left(w_{e}, w_{h}, T^{\mathrm{eq}}\right)$ are precalculated for the reservoir carrier densities and temperature commonly encountered in the quantum-dot laser and implemented as look-up tables in the simulations to minimize computational effort.

The electric field dynamics are written as

$$
\frac{\mathrm{d}}{\mathrm{d} t} E=(g(t)-\kappa) E+\left.\frac{\partial}{\partial t} E\right|_{\mathrm{sp}} .
$$

with the complex optical gain given by the sum over all quantum-dot subgroup gain coefficients,

$$
g_{m}^{j}=\frac{T_{2}\left|\mu_{m}\right|^{2}}{2 \hbar^{2}}\left(\frac{1-i T_{2}\left(\omega_{m}^{j}-\omega\right)}{1+\left[T_{2}\left(\omega_{m}^{j}-\omega\right)\right]^{2}}\right) \text {, }
$$


as well as the frequency shift induced by the quantum-well transitions in quasiequilibrium approximation,

$$
\delta \omega_{\mathrm{QW}}=-\frac{\hbar \omega \Gamma}{\varepsilon_{0} \varepsilon_{\mathrm{bg}} h^{\mathrm{QW}}} \frac{2}{A_{\mathrm{act}}} \sum_{\boldsymbol{k}^{2 \mathrm{D}}} \operatorname{Im} g_{\boldsymbol{k}}^{2 \mathrm{D}}\left(f\left(\varepsilon_{e, \boldsymbol{k}}^{2 \mathrm{D}}, E_{\mathrm{F}, e}^{\mathrm{eq}}, T^{\mathrm{eq}}\right)+f\left(\varepsilon_{h, \boldsymbol{k}}^{2 \mathrm{D}}, E_{\mathrm{F}, h}^{\mathrm{eq}}, T^{\mathrm{eq}}\right)-1\right) .
$$

The gain can then be written as

$$
g(t)=\frac{\hbar \omega \Gamma}{\varepsilon_{0} \varepsilon_{\mathrm{bg}} h^{\mathrm{QW}}} 2 N^{\mathrm{QD}} \sum_{j, m} \nu_{m} f(j) g_{m}^{j}\left(\rho_{e, m}^{j}(t)+\rho_{h, m}^{j}(t)-1\right)-i \delta \omega_{\mathrm{QW}} .
$$

The spontaneous emission is included deterministically, by using

$$
\left.\frac{\partial}{\partial t} E(t)\right|_{\mathrm{sp}}=\frac{1}{2 E^{*}} \frac{4 \beta \Gamma N^{\mathrm{QD}}}{\varepsilon_{\mathrm{bg}} \varepsilon_{0} h^{\mathrm{QW}}} \sum_{j, m} \nu_{m} f(j) W_{m} \rho_{e, m}^{j} \rho_{h, m}^{j} \hbar \omega_{m}^{j} .
$$

The charge-carrier distribution temperature is calculated dynamically from the energy density balance equations as derived in Se. 2.5.2

$$
\frac{\mathrm{d}}{\mathrm{d} t} u=\left.\frac{\mathrm{d} u}{\mathrm{~d} t}\right|_{\text {pump }}+\left.\frac{\mathrm{d} u}{\mathrm{~d} t}\right|_{\text {rec }}+\left.\frac{\mathrm{d} u}{\mathrm{~d} t}\right|_{\text {Auger }}+\left.\frac{\mathrm{d} u}{\mathrm{~d} t}\right|_{\text {phon }} .
$$

The above coupled differential equation system describes the complete dynamics of the quantum-dot laser active medium with a separate treatment of electrons and holes in the inhomogeneously broadened first two localized quantum-dot states as well as in the surrounding quasi-two-dimensional charge carrier reservoir, taking microscopically calculated Auger-scattering rates into account. The carrier-induced gain and refractive index change is calculated separately for each optical transition, driving the light-matter interaction.

In the following sections the above equations are solved numerically, using the simulation parameters given in Table 3.1, and the quantum-dot structure as illustrated in Fig. 3.2.

Figure 3.2: Energy scheme of the localized quantum-dot states, unless stated otherwise. The energy spacings are given in meV. The reservoir band edges are shown by the light-blue shaded areas.

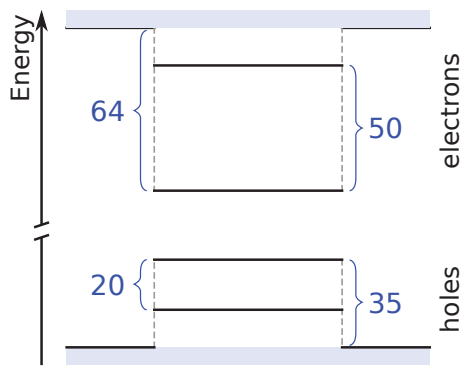


Table 3.1.: Parameters used in the quantum-dot laser simulations, unless stated otherwise.

\begin{tabular}{lll}
\hline \hline SYMBOL & VALUE & MEANING \\
\hline$N^{\mathrm{QD}}$ & $10^{11} \mathrm{~cm}^{-2}$ & QD density per layer \\
$a_{L}$ & 15 & number of layers \\
$h^{\mathrm{QW}}$ & $5 \mathrm{~nm}$ & QW layer height \\
$n_{\mathrm{bg}}$ & 3.77 & background index \\
$\Delta E_{\mathrm{inh}}$ & $40 \mathrm{meV}^{2}$ & QD inhomogeneous broadening FWHM \\
$B^{S}$ & $540 \mathrm{~nm}^{2} \mathrm{~ns}^{-1}$ & QW bimolecular recombination rate \\
$W_{\mathrm{GS}}$ & $0.44 \mathrm{~ns}^{-1}$ & GS spontaneous recombination rate \\
$W_{\mathrm{ES}}$ & $0.55 \mathrm{~ns}^{-1}$ & ES spontaneous recombination rate \\
$\beta$ & $2 \times 10^{-3}$ & spontaneous emission ratio \\
$\mu_{m}$ & $0.6 \mathrm{~nm}_{0}$ & QD transition dipole moment \\
$\mu_{\mathrm{QW}}$ & $0.5 \mathrm{~nm}_{0}$ & QW transition dipole moment \\
$T_{2}$ & $100 \mathrm{fs}$ & QD polarization dephasing time \\
$\Gamma$ & 0.03125 & Geometric confinement factor \\
$\kappa$ & $50 \mathrm{~ns}^{-1}$ & optical loss rate \\
$\gamma_{p}$ & $0.2 \mathrm{ps}^{-1}$ & carrier-phonon interaction rate \\
$\varepsilon_{\mathrm{pump}}$ & $0.23 \mathrm{eV}$ & charge carrier pump energy \\
$\Delta E_{e}\left(\Delta E_{h}\right)$ & $64 \mathrm{meV}(35 \mathrm{meV})$ & electron (hole) QD GS localization energy \\
$\Delta_{e}\left(\Delta_{h}\right)$ & $50 \mathrm{meV}(20 \mathrm{meV})$ & electron (hole) QD GS-ES energy spacing \\
\hline \hline
\end{tabular}

The light-current characteristic of the modeled quantum-dot laser device is shown in Fig. 3.3, along with the steady-state charge-carrier densities per quantum-well layer in the quantum-dot and quantum-well reservoir states:

$$
\begin{aligned}
n_{m}^{\mathrm{QD}} & =2 N^{\mathrm{QD}} \sum_{j} \nu_{m} f(j)\left(\rho_{e, m}^{j}+\rho_{h, m}^{j}\right) \\
n^{\mathrm{QW}} & =w_{e}+w_{h} .
\end{aligned}
$$

After the threshold current is reached, the output power increases linearly with the pump current and the quantum-dot ground-state carrier density is clamped to its threshold value. These characteristics closely resemble the behavior of the simple two-variable rate-equation system, Eqs. (3.1). The off-resonant charge carriers in the excited and reservoir states, however, keep increasing above their threshold values, shown by the dashed horizontal lines in Fig. 3.3. This is a direct consequence of the scattering processes filling the active quantum-dot states: In the steady state the ground-state charge carriers lost in stimulated emission processes, i.e., the lasing action, must be replenished by the in-scattering of charge carriers from the other states. Since the laser intensity increases with current, the increase in stimulated losses must be compensated by higher in-scattering of charge carriers, which thus necessitates higher carrier occupations in the other states. The charge carrier occupations in quantum-dot lasers are therefore not clamped [LUE10], in 


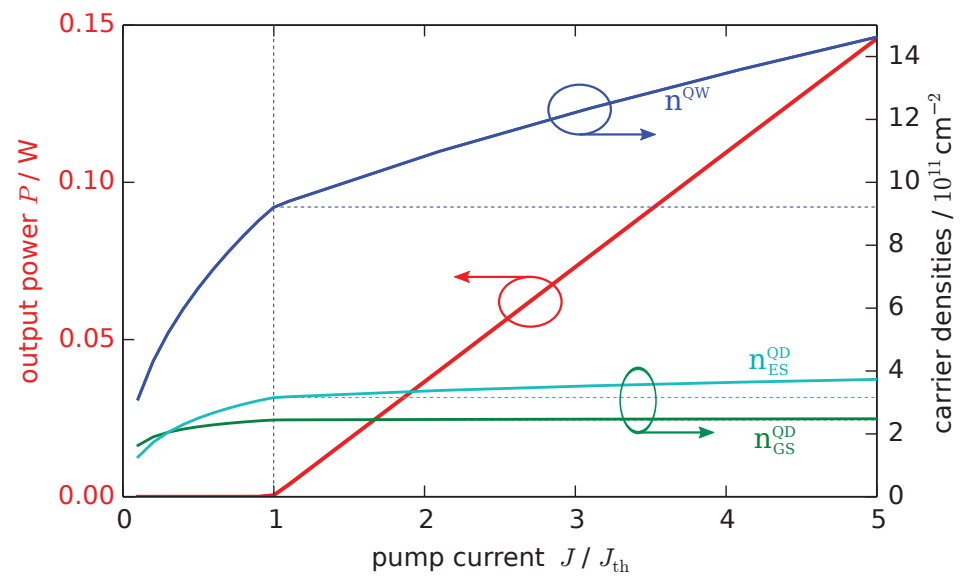

Figure 3.3.: Simulated quantum-dot laser light-current characteristics. Shown is the laser output power (red), total quantum-dot ground-state (GS) carrier density (green), quantum-dot excited-state (ES) density (cyan) and quantum-well (QW) density (blue) in each QW layer, all in dependence on the pump current normalized to the threshold current $J_{\mathrm{th}}$. The horizontal dashed lines show the respective values at the threshold current.

contrast to simpler laser models. This will lead to important differences in the dynamical behavior of quantum-dot laser compared to conventional lasers, as will be shown in the following sections.

The steady-state characteristics shown in Fig. 3.3 depict the dependence of the stationary steady-state values of the output power and carrier densities. For a characterization of the dynamic properties, the time-dependence of the laser variables must be investigated. We thus integrate the quantum-dot laser equations in time after the onset of the pump current. The resulting turn-on dynamics at a pump current of twice the threshold current $J_{\text {th }}$ are shown in Fig. 3.4.

The intensity time-series, Fig. $3.4 \mathrm{a}$, reveals an intensity peak after a turn-on delay time of about $600 \mathrm{ps}$ and subsequent strongly damped relaxation oscillations. This comparably high damping is characteristic of quantum-dot lasers and has been related to the charge-carrier scattering dynamics between the localized quantumdot and reservoir states as a limiting factor [HEI01, KUN02, ISH07, LUE10]. The turn-on trajectory plotted in the phase-space spanned by the output power and the total quantum-dot ground-state charge-carrier density $\left(n_{e}^{\mathrm{QD}}+n_{h}^{\mathrm{QD}}\right)$ in Fig. $3.4 \mathrm{~b}$ show a qualitative similar behavior as the simple two-variable rate equations shown in Fig. 3.1 b, i.e., the fixed point is a stable focus as well, albeit with much higher damping. 

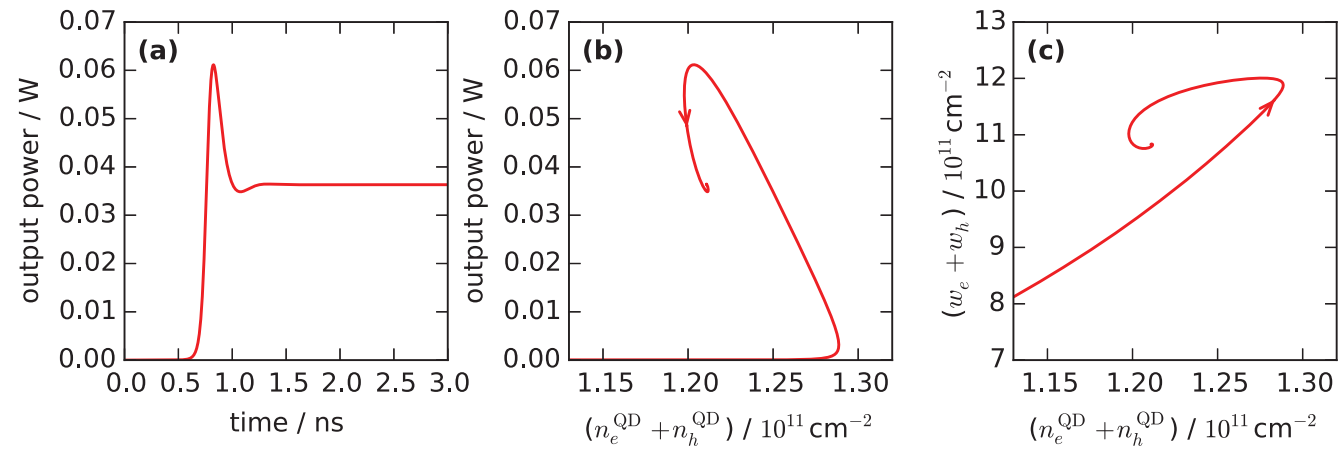

Figure 3.4.: Simulated quantum-dot laser turn-on dynamics. (a) Time-series of the output power after onset of the pump current at $t=0$. (b) Phase-space trajectory in the phase-space spanned by total quantum-dot ground-state carrier density and output power. (c) Phase-space trajectory in the phase-space spanned by total quantum-dot carrier density and total reservoir carrier density.

The phase-space plot in Fig. 3.4c shows the trajectory in the quantum-dot quantum-well charge-carrier density phase-space. Here, also a spiraling motion into the fixed point is visible. This reveals a phase-shift between the oscillations in the corresponding charge-carrier ensembles, i.e., the carrier reservoir and quantum-dot carriers exhibit desynchronized dynamics. A constant quasi-equilibrium between the different charge-carrier sub-ensembles would translate to a functional dependence of $n_{b}^{\mathrm{QD}}$ on $w_{b}$, and vice versa, from which the shown result (Fig. 3.4) clearly deviates. The nonequilibrium description of the charge carriers in quantum-dot devices is therefore required and leads to important dynamic effects, as will be shown in the following sections.

\subsubsection{Influence of Charge-Carrier Scattering}

As detailed before, the charge-carrier scattering processes in quantum-dot lasers are an important aspect that distinguishes them from conventional quantum-well or bulk lasers. We will therefore investigate the dependence of the quantum-dot laser dynamics on the timescales of the involved scattering processes. The exact numeric value of the scattering rates can depend on the operating current and temperature [MAJ11], the material composition [NIE04, NIE05], the quantum-dot size, or the dimensionality of the carrier reservoir [WIL12b]. The scattering rates can therefore vary between different devices and applications. In order to understand the quantum-dot laser dynamics, a systematic study of the influence of the scattering processes is thus needed. 
The scattering contribution to the quantum-dot ground-state carrier occupation is given by

$$
\begin{aligned}
\left.\frac{\mathrm{d}}{\mathrm{d} t} \rho_{b, \mathrm{GS}}^{j}\right|_{\mathrm{sc}}= & S_{b, \mathrm{GS}}^{j, \mathrm{cap}}+S_{b}^{j, \text { rel }} \\
= & \left(S_{b, \mathrm{GS}}^{\mathrm{cap}, \mathrm{in}}+S_{b}^{\mathrm{rel}, \text { in }} \rho_{b, \mathrm{ES}}^{j}\right)\left(1-\rho_{b, \mathrm{GS}}^{j}\right) \\
& -\left(S_{b, \mathrm{GS}}^{\mathrm{cap}, \text { out }}+S_{b}^{\mathrm{rel}, \mathrm{out}}\left(1-\rho_{b, \mathrm{ES}}^{j}\right)\right) \rho_{b, \mathrm{GS}}^{j} \cdot
\end{aligned}
$$

Looking now at a small deviation $\delta \rho$ from the steady-state value of $\rho_{b, \mathrm{GS}}^{j}$, the above equation can be linearized in $\rho_{b, \mathrm{GS}}^{j}$ and rewritten as

$$
\frac{\mathrm{d}}{\mathrm{d} t} \delta \rho=-\frac{\delta \rho}{\tau_{b}},
$$

where the effective scattering lifetime $\tau_{b}$ was introduced, given by

$$
\tau_{b}{ }^{-1}=S_{b, \mathrm{GS}}^{\mathrm{cap}, \text { in }}+S_{b, \mathrm{GS}}^{\mathrm{cap}, \text { out }}+S_{b}^{\mathrm{rel}, \mathrm{in}} \rho_{b, \mathrm{ES}}^{j}+S_{b}^{\mathrm{rel}, \text { out }}\left(1-\rho_{b, \mathrm{ES}}^{j}\right),
$$

which allows for a characterization of the scattering processes by a single value. The scattering processes will thus drive the occupation after a small perturbation back towards the steady-state value, with a time constant given by this effective scattering lifetime. This makes $\tau_{b}$ experimentally accessible, e.g, by pump-probe measurements of the gain recovery of quantum-dot lasers or amplifiers, which measure the time-dependence of the optical gain after an optical excitation which depletes the initial carrier occupation of the system [BOR00, DOM07, PIW07, GOM08, KAP14c]. Provided the initial depletion is not too large, the subsequent gain recovery can be fitted by a single-exponential recovery, which then yields the effective scattering lifetime as the time constant. For strong perturbation, however, the linearization approach used to derive $\tau_{b}$ no longer holds, and also the charge carriers in the excited and reservoir states will be depleted, which introduces a nonlinear recovery, as the individual scattering rates depend on these occupations.

We now proceed by analyzing the quantum-dot laser dynamics in dependence on the scattering rates. In order to isolate their effects, we start from the microscopically calculated rates and introduce a common constant scaling factor to all individual scattering rates. As before, we simulate the turn-on dynamics of the quantum-dot laser with three different scaling factors, leading to effective scattering lifetimes $\tau_{b}$ in the steady-states. The resulting turn-on curves are shown in Fig. 3.5 a. Both for the fast scattering $\tau_{e}^{-1}=10^{12} \mathrm{~s}^{-1}$ (red) and the slow value $\tau_{e}^{-1}=10^{10} \mathrm{~s}^{-1}$ (blue) the laser exhibits relatively strong relaxation oscillations after turn-on. For intermediate rates, the quantum-dot laser exhibits an overdamped 

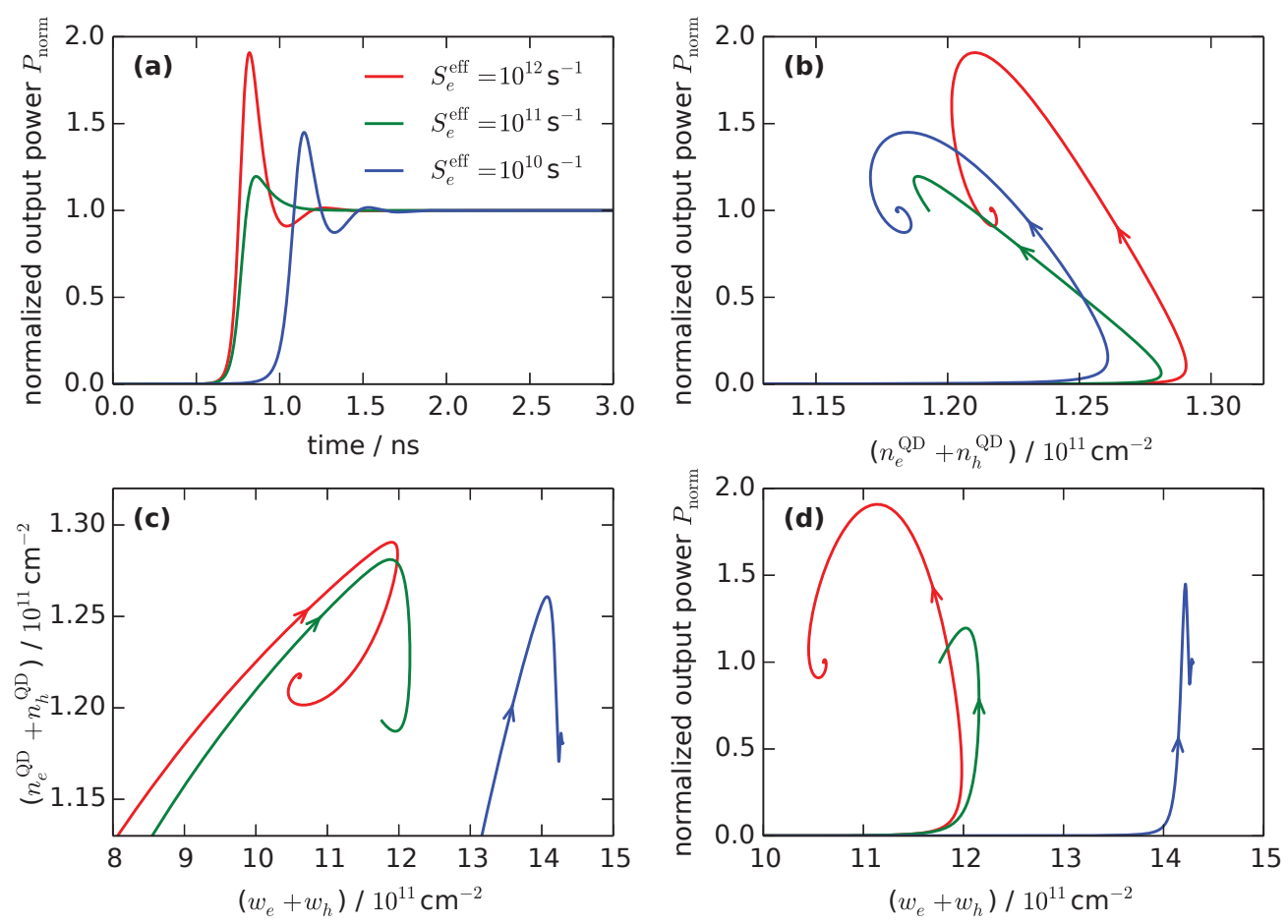

Figure 3.5.: Simulated quantum-dot laser turn-on dynamics for fast (red), intermediate (green), and slow (blue) scattering. (a) Time-series of the normalized output power $P_{\text {norm }}$ after onset of the pump current at $t=0$. (b) Phase-space trajectory in the phasespace spanned by total quantum-dot carrier density and output power. (c) Phase-space trajectory in the phase-space spanned by total quantum-dot carrier density and total reservoir carrier density. (d) Phase-space trajectory in the phase-space spanned by total reservoir carrier density and the normalized output power. The laser was biased at twice the threshold current in each case. After [LIN12].

response, with a steady decay of the output power towards the steady-state value without oscillations.

Taking into account the expression for the relaxation oscillation frequency and damping determined from the simple two-variable rate-equation system, Eq. (3.7), the damping could be expected to grow linearly with the inverse carrier lifetime $T_{1}^{-1}$. As the effective scattering lifetime enters the linearized equation Eq. (3.18) in the same form as $T_{1}$ in Eqs. (3.1), the damping could be expected to grow with $\tau_{e}^{-1}$, which is also supported by analytical treatment of more sophisticated quantum-dot models [LUE11]. The corresponding behavior can be observed when increasing the scattering rates from the slow to intermediate scattering cases in Fig. $3.5 \mathrm{a}$, where the damping increases. The reoccurring relaxation oscillations at even faster scattering, however, is not predicted by the simple model [ERN07a]. 
In order to understand this behavior, it is useful to analyze the turn-on process in different projections of the phase-space, shown in Fig. $3.5 \mathrm{~b}-\mathrm{d}$. Here, the trajectory in the phase-space spanned by the quantum-dot carrier density $\left(n_{e}^{\mathrm{QD}}+n_{h}^{\mathrm{QD}}\right)$ and normalized output power $P_{\text {norm }}$ shows a spiraling motion or a simple linear motion towards the steady-state values for the oscillating and overdamped cases, respectively. This transition corresponds to a change of the fixed point from a stable focus to a stable node. The trajectories in Fig. $3.5 \mathrm{c}-\mathrm{d}$ involving the carrier reservoir carrier densities $\left(w_{e}+w_{h}\right)$ reveal the nature of the observed turn-on behavior. For the slow scattering, the motion in the $\left(w_{e}+w_{h}, P_{\text {norm }}\right)$-phase-space shows a very different behavior from the $\left(n_{e}^{\mathrm{QD}}+n_{h}^{\mathrm{QD}}, P_{\text {norm }}\right)$-phase-space. During the whole turn-on, the reservoir carrier density continues to rise. The interaction with the photons during the relaxation oscillations is thus limited to the quantumdot carriers, while the reservoir carriers are not affected. We therefore refer to this regime of slow scattering as the "constant-reservoir regime".

With increasing scattering rates, the reservoir starts to be affected by the lightmatter interaction in the quantum-dots, manifesting itself in qualitative similar dynamics of the quantum-dot and reservoir carriers. For very fast scattering, the different carriers are closely coupled, and perturbations to the quantum-dot population will be transmitted to the reservoir population. In the limit of instantaneous scattering, i.e., $\tau_{b} \rightarrow 0$, the reservoir carriers would adiabatically follow the quantum-dot occupation. The dynamic degree of freedom of the reservoir and excited state carriers could then be adiabatically eliminated and the carriers would behave as a combined charge-carrier system, and a single variable would suffice to describe all charge carrier occupations. The quantum-dot laser dynamics could then be described to good accuracy by a simple rate-equation system similar to quantum-well laser models. As shown for the two-variable laser model Eqs. (3.1) before, the relaxation oscillation damping in this case is given by the carrier lifetime related to the non-radiative losses, which are in the order of ns, leading to the reappearance of relaxation oscillations for the fast scattering. We call this dynamic regime of fast scattering the "synchronized" regime, where the different carrier sub-ensembles are strongly coupled. Here, the quantum-dot laser dynamics approach that of quantum-well lasers [ERN07a].

For a detailed study of the quantitative dependence of the quantum-dot laser dynamics on the scattering rates, we extract the relaxation oscillation frequency and damping in dependence of the scattering lifetime. Numerically, this is done by fitting the output power time-series after a small perturbation with a damped harmonic oscillation (see Appendix A.1). The resulting relaxation oscillation parameters in dependence on the averaged effective carrier scattering rate, $S^{\text {eff }} \equiv$ $\frac{1}{2}\left(\tau_{e}^{-1}+\tau_{h}^{-1}\right)$, is shown in Fig. 3.6. 


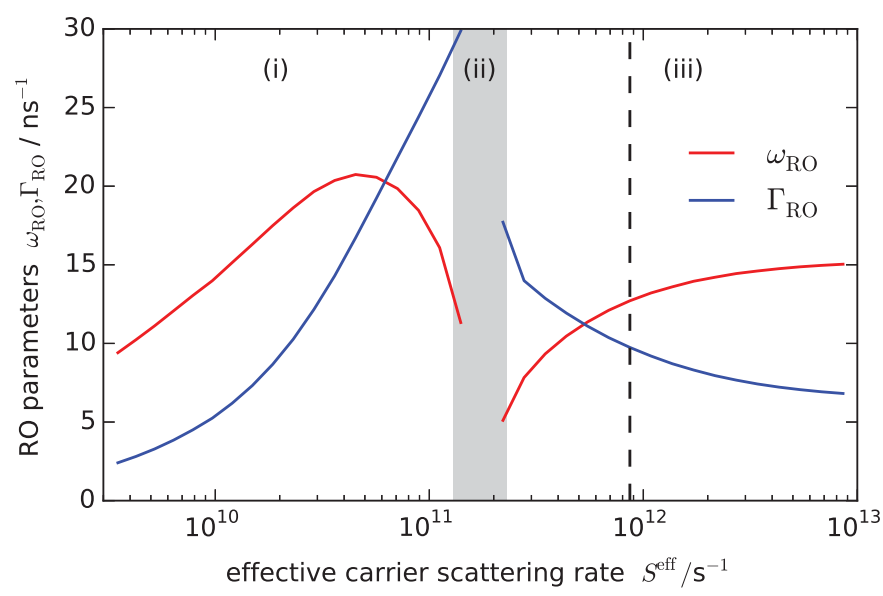

Figure 3.6.: Relaxation oscillation $(\mathrm{RO})$ parameters in dependence of the scattering rates. Shown is the RO frequency $\omega_{\mathrm{RO}}$ and the RO damping $\Gamma_{\mathrm{RO}}$ for different effective carrier scattering rates $S^{\mathrm{eff}} \equiv \frac{1}{2}\left(\tau_{e}^{-1}+\tau_{h}^{-1}\right)$, averaged over electrons and holes. (i) Constantreservoir regime, (ii) overdamped regime, (iii) synchronized regime. The pump current was set to twice the respective threshold current at each data point. The dashed vertical line denotes the microscopically calculated scattering rates for the device parameters given in Table 3.1. After [LIN12, LIN14].

Clearly visible are the three qualitatively different dynamic regimes. The relaxation oscillation frequency vanishes around $S^{\text {eff }} \approx 1.5 \times 10^{11} \mathrm{~s}$ and the damping becomes maximal, indicating the overdamped regime. Changing the scattering rates from there to either lower or higher values, the damping decreases and the relaxation oscillations reappear. The microscopically calculated scattering rates lie in the synchronized regime, close to the overdamped regime, such that the coupling between quantum-dot and reservoir states is strong enough for the reservoir states to be affected by the laser dynamics. On the other hand, the scattering is not fast enough as to lead to a near-instantaneous coupling of the two carrier sub-ensembles, therefore still allowing for independent carrier dynamics. 


\subsection{Minimal Model for Quantum-Dot Laser Dynamics}

The crucial dependence of the quantum-dot dynamics on the involved scattering rates can not be described by the traditional rate-equation approach given by Eqs. (3.1), as seen in the previous section. An important task is therefore to find the effects responsible for the strong differences between the two models. By pinpointing these physical processes, one can then formulate requirements for a given model to be able to describe the physical phenomena in quantum-dot lasers. This way it becomes possible to derive a minimal model taking all these effects into account, which can then qualitatively describe the quantum-dot laser dynamics. The derivation of such a simple model will be done in this section.

As we have stressed before, the most important difference between conventional, e.g., quantum-well lasers and quantum-dot devices is the complex charge carrier scattering dynamics between the optically active states and the surrounding carrier reservoir. An intuitive approach in order to extend the existing rate-equation system Eq. (3.1) to quantum-dot devices is to introduce the quantum-dot occupation probability, and taking the charge carrier density $N$ as the reservoir charge carrier density. The resulting three-variable system can be written as

$$
\begin{aligned}
\frac{\mathrm{d}}{\mathrm{d} t} N & =J-\frac{N}{T_{1}}-\widetilde{S}(N, \rho) \\
\frac{\mathrm{d}}{\mathrm{d} t} \rho & =\widetilde{S}(N, \rho)-\frac{\rho}{T_{\mathrm{sp}}}-g(2 \rho-1) S \\
\frac{\mathrm{d}}{\mathrm{d} t} S & =2 g(2 \rho-1) S-2 \kappa S,
\end{aligned}
$$

where the coupling between $\rho$ and $N$ is for now expressed as a general scattering term $\widetilde{S}(N, \rho)$. The variable $N$ expresses the reservoir charge-carrier density in units of $2 N^{\mathrm{QD}}$. The spontaneous losses in the quantum-dot are taken into account by the spontaneous loss time $T_{\mathrm{sp}}$, the stimulated emission is given by the gain coefficient $g$ and the inversion $(2 \rho-1)$.

We now need to define the scattering contribution $\widetilde{S}(N, \rho)$. For direct capture processes, i.e., the charge carrier scattering between the quantum-dot and reservoir states, the scattering can be written as

$$
\widetilde{S}(N, \rho)=S^{\text {cap }, \text { in }}(N)(1-\rho)-S^{\text {cap }, \text { out }}(N) \rho .
$$

We rewrite this expression as [LIN12]

$$
\widetilde{S}(N, \rho)=R(N)\left(\rho^{\mathrm{eq}}(N)-\rho\right),
$$


with

$$
R(N) \equiv S^{\text {cap ,in }}+S^{\text {cap }, \text { out }} \quad \rho^{\text {eq }}(N) \equiv \frac{S^{\text {cap }, \text { in }}}{S^{\text {cap,in }}+S^{\text {cap }, \text { out }}}
$$

The scattering processes can thus be described as driving the quantum-dot population towards the quasi-equilibrium occupation probability $\rho^{\mathrm{eq}}$. The individual rates depend on the carrier-reservoir density, and thus also $\rho^{\mathrm{eq}}$. This dependence is given by the detailed balance relationship,

$$
\begin{aligned}
S^{\text {cap }, \text { out }} & =S^{\text {cap }, \text { in }} \exp \left(\frac{\varepsilon^{\mathrm{QD}}-E_{F}^{\mathrm{eq}}}{k_{\mathrm{B}} T}\right) \\
& =S^{\mathrm{cap}, \text { in }} \exp \left(\frac{\varepsilon^{\mathrm{QD}}-E_{0}^{\mathrm{QW}}}{k_{\mathrm{B}} T}\right)\left[\exp \left(\frac{w}{\mathcal{D} k_{\mathrm{B}} T}\right)-1\right]^{-1},
\end{aligned}
$$

with the quasi-Fermi level in the reservoir $E_{F}^{\mathrm{eq}}$, the reservoir band edge $E_{0}^{\mathrm{QW}}$ and the quantum-dot energy level $\varepsilon^{\mathrm{QD}}$, as well as the reservoir carrier density $w=2 N^{\mathrm{QD}} \cdot N$ and the corresponding density of states $\mathcal{D}$. This expression leads to a rather complicated dependence of the scattering term on the reservoir density. Thus, in order to simplify the resulting terms, we linearize $\rho^{\text {eq }}$ in terms of the reservoir carrier density. We choose a linearization around the corresponding threshold values of $N$ and $\rho$. As we have seen previously, the charge-carrier densities in quantum-dot lasers are not clamped above threshold. Nevertheless, the deviations from the threshold values can be seen as small, such that the linearization should yield reliable results. Furthermore, the threshold values $N^{\text {th }}, \rho^{\text {th }}$ have an intuitive physical interpretation: $N^{\text {th }}$ is the number of charge carriers per quantum-dot in the reservoir required to reach the lasing threshold. The threshold occupation probability $\rho^{\text {th }}$ on the other hand can be easily calculated from the lasing condition, i.e. $\frac{\mathrm{d}}{\mathrm{d} t} S \equiv 0$. We can thus write

$$
\rho^{\mathrm{eq}} \approx \rho^{\mathrm{th}}+d\left(N-N^{\mathrm{th}}\right)
$$

with

$$
\rho^{\text {th }} \equiv \frac{g+\kappa}{2 g},\left.\quad d \equiv \frac{\partial}{\partial N}\left[\frac{S^{\text {cap }, \text { in }}}{S^{\text {cap,in }}+S^{\text {cap }, \text { out }}}\right]\right|_{N^{\text {th }}} .
$$

The newly introduced coefficient $d$ thus describes the change of the quasi-equilibrium quantum-dot occupation with the reservoir carrier density, due to the change in 
the detailed balance between in and out-scattering. With these expressions, the three-variable system for the quantum-dot laser is written as:

$$
\begin{aligned}
\frac{\mathrm{d}}{\mathrm{d} t} N & =J-\frac{N}{T_{1}}-R\left(\rho^{\mathrm{th}}+d\left(N-N^{\mathrm{th}}\right)-\rho\right) \\
\frac{\mathrm{d}}{\mathrm{d} t} \rho & =R\left(\rho^{\mathrm{th}}+d\left(N-N^{\mathrm{th}}\right)-\rho\right)-\frac{\rho}{T_{\mathrm{sp}}}-g(2 \rho-1) S \\
\frac{\mathrm{d}}{\mathrm{d} t} S & =2 g(2 \rho-1) S-2 \kappa S .
\end{aligned}
$$

\subsubsection{Linearization and Eigenvalue Problem}

In the following, the derived rate-equation system will be analyzed in terms of its relaxation oscillation damping and frequency. To this end, we will perform a linear stability analysis around its fixed point. Subsequently, the dependence on the effective scattering rate $R$ will be analyzed.

The lasing fixed point of the three-variable equation system Eqs. (3.27) is given by

$$
N^{*}=\frac{T_{1}\left(J+d R N^{\mathrm{th}}\right)}{1+d R T_{1}}, \quad \rho^{*}=\frac{g+\kappa}{2 g}, \quad S^{*}=\frac{d R}{\kappa}\left(N^{*}-N^{\mathrm{th}}\right)-\frac{\rho^{*}}{\kappa T_{\mathrm{sp}}} .
$$

In order to determine the eigenvalue problem we determine the Jacobian matrix

$$
\underline{J}=\left(\begin{array}{ccc}
-\frac{1}{T_{1}}-d R & R & 0 \\
d R & -\frac{1}{T_{\mathrm{sp}}}-R-2 g S^{*} & -\kappa \\
0 & 4 g S^{*} & 0
\end{array}\right),
$$

from which we can determine the eigenvalues as the roots of the characteristic equation

$0=-\lambda\left[\left(\frac{1}{T_{1}}+d R+\lambda\right)\left(\frac{1}{T_{\mathrm{sp}}}+R-2 g S^{*}+\lambda\right)-d R^{2}\right]-4 g \kappa S^{*}\left(\frac{1}{T_{1}}+d R+\lambda\right)$.

The roots of above equation, however, are very complex expressions which can not be used for an analytic treatment of the problem. In order to determine the stability properties and Lyapunov-exponents of the fixed points, the solutions to the above equations will be determined numerically.

We choose parameters as given in Table 3.2. These parameters were chosen in correspondence with those given in Table 3.1 for the full quantum-dot laser model, 
Table 3.2.: Parameters used in the minimal quantum-dot laser model, unless stated otherwise.

\begin{tabular}{lll}
\hline \hline Symbol & VAluE & MeANing \\
\hline$T_{1}$ & $0.17 \mathrm{~ns}$ & reservoir charge carrier lifetime \\
$T_{\mathrm{sp}}$ & $1.85 \mathrm{~ns}$ & quantum-dot charge carrier lifetime \\
$g$ & $230 \mathrm{~ns}^{-1}$ & gain coefficient \\
$\kappa$ & $50 \mathrm{~ns}^{-1}$ & optical losses \\
$N^{\text {th }}$ & 2.3 & reservoir carrier density at threshold (in units of $2 N^{\mathrm{QD}}$ ) \\
$d$ & 0.022 & detailed balance coefficient \\
\hline \hline
\end{tabular}

evaluated at twice the threshold current. The effective charge carrier lifetimes are given by

$$
T_{1}=\left[B^{S}\left(w_{e}+w_{h}\right)\right]^{-1}, \quad T_{\mathrm{sp}}=\left[W_{\mathrm{GS}}\left(\overline{\rho_{e, \mathrm{GS}}}+\overline{\rho_{h, \mathrm{GS}}}\right)\right]^{-1},
$$

with the average quantum-dot ground-state occupations $\overline{\rho_{b, \mathrm{GS}}}$. The eigenvalues in the steady-state are determined numerically. The resulting dependence on the effective scattering rate $R$ is shown in Fig. 3.7. The minimal model reproduces the three qualitative dynamic regimes that have been previously found for the

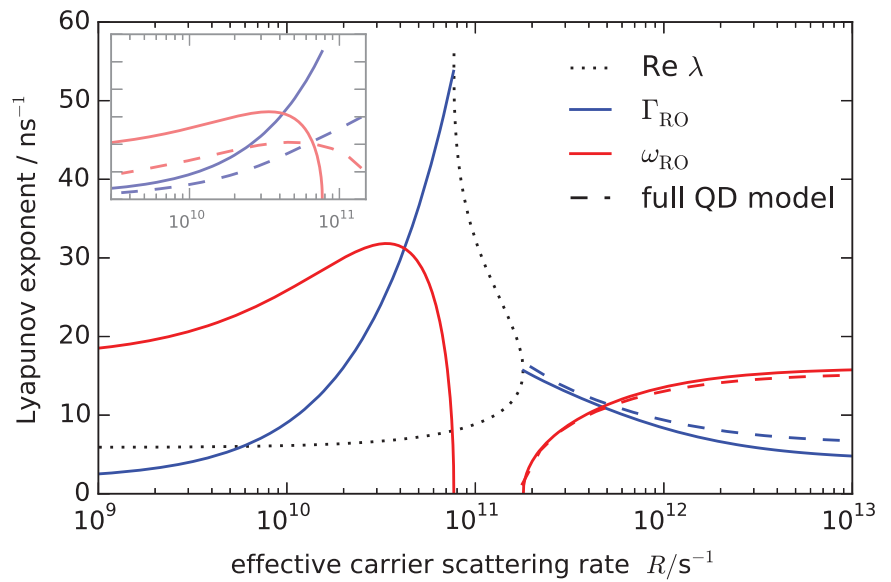

Figure 3.7.: Eigenvalues $\lambda$ in the minimal quantum-dot model. Shown are the relaxation oscillation $(\mathrm{RO})$ damping $\Gamma_{\mathrm{RO}}\left(\equiv \operatorname{Re} \lambda\right.$, blue) and frequency $\omega_{\mathrm{RO}}(\equiv \operatorname{Im} \lambda$, red) of the eigenvalues that are complex conjugate pairs. The dotted black line shows the eigenvalues that are not related to the relaxation oscillations. The dashed lines show the results from the full quantum-dot laser model. The inset shows the comparison in the constant-reservoir regime. The pump current was set to twice the respective threshold value at each data point. 
quantum-dot laser: two regimes with pronounced relaxation oscillations for low and high $R$, with an intermediate overdamped regime around $R \approx 10^{11} \mathrm{~s}^{-1}$. In the synchronized regime for large $R$ also the results from the full quantum-dot laser model are shown as dashed lines. Here, a very good quantitative agreement between the two approaches is visible. For slower scattering, however, while the three-variable model correctly predicts the qualitative dependence of the dynamic regimes on the scattering rate, the two approaches differ (see inset). There are possible reasons for this discrepancy:

- The three-variable rate-equation model does not take into account the different dynamics of electrons and holes. Especially for slow scattering this can introduce additional nonlinearities, as their dynamics then become less synchronized [LUE09].

- For slow scattering the reservoir densities deviate from their respective threshold values, as higher reservoir densities are required to supply the quantumdot states with enough charge carriers. Thus, the linearization approximations may not hold any longer.

- Further effects, such as gain compression, which are especially pronounced for slow charge carrier scattering [CHO99, FIO07], have not been addressed in the simple model. Gain compression is known to influence the relaxation oscillation parameters [COL12a], and might thus change the shown dependence for low $R$.

One must therefore keep in mind that the derived model should not be used for a quantitative description of a given quantum-dot laser device. Nevertheless it already offers a much better description than the conventional two-variable rateequation model. At a given operation point a correct choice of the effective model parameters, e.g., from comparisons with experiments, should also yield quantitatively reliable results.

In contrast to three-variable models for quantum-dot lasers that do not take into account the detailed balance of the scattering rates [ERN07a], our model is able to reproduce the reappearance of relaxation oscillations in the synchronized dynamic regime for high scattering rates. As we have seen this is a direct consequence of a reservoir-carrier-density dependent out-scattering rate.

Furthermore, the derived model can yield important analytical results. By applying additional model reductions an analytical treatment and formulation of closedform expressions become possible, e.g., for the relaxation oscillation parameters. This will be done in the following. 


\subsubsection{Asymptotic Analysis - Relaxation Oscillations}

The characteristic equation Eq. (3.30) needs to be solved in order to calculate the eigenvalues of the Jacobian and thus the stability as well as relaxation oscillation parameters. Given its cubic nature, the solutions can in principle be explicitly determined. However, this would result in lengthy and complicated expressions with little practical value, as the dependences on different parameters would be hidden in the multitude of terms. The idea to circumvent these issues is to expand the characteristic equation in terms of one or more small parameters, and solve the reduced problem [ERN10b, LUE11]. Depending on the choice of expansion, the resulting eigenvalues should describe the original system well around the chosen expansion point or parameter.

\section{Slow Scattering - Constant-Reservoir Regime}

At first, we will try to derive a simpler expression for the eigenvalues in the constant-reservoir regime. Our previous numerical analysis of the quantum-dot laser dynamics within this regime has shown that the dynamics are dominated by the quantum-dot charge-carrier dynamics, while the reservoir provides a nearly constant charge carrier influx into the active quantum-dot states. As such, the charge-carrier subsystems should be only weakly coupled. This leads us to a choice of expansion: In the given three-variable system, the direct coupling between the quantum-dot and reservoir carriers is determined by the coefficient $d$, describing the change of quasi-equilibrium occupation in the quantum-dots depending on the reservoir carrier density.

We will therefore expand Eq. (3.30) in terms of $d$. We assume that the eigenvalues can be written in the form

$$
\lambda=\lambda_{0}+d \lambda_{1}+O\left(d^{2}\right) .
$$

We proceed by inserting this into Eq. (3.30). Since our assumption was that $d$ should be small, we can at first neglect all resulting terms of order $d$ or higher, as the remaining terms should dominate the dynamics. This zeroth-order problem can then be explicitly solved for $\lambda_{0}$.

The resulting zeroth-order characteristic equation is given by

$$
\begin{aligned}
0= & 4 g \kappa S^{*} T_{\mathrm{sp}} \\
& +\left(1+R T_{\mathrm{sp}}+2 g S^{*} T_{\mathrm{sp}}+4 g \kappa S^{*} T_{1} T_{\mathrm{sp}}\right) \lambda_{0} \\
& +\left(T_{1}+T_{\mathrm{sp}}+R T_{1} T_{\mathrm{sp}}+2 g S^{*} T_{1} T_{\mathrm{sp}}\right) \lambda_{0}{ }^{2} \\
& +T_{1} T_{\mathrm{sp}} \lambda_{0}^{3}
\end{aligned}
$$


which has three solutions,

$$
\begin{aligned}
\lambda_{0} & =-\Gamma_{\mathrm{RO}}^{\mathrm{cr}, 0} \pm i \omega_{\mathrm{RO}}^{\mathrm{cr}, 0}, \\
\lambda_{0^{*}} & =\frac{1}{T_{1}},
\end{aligned}
$$

with the zeroth-order relaxation oscillation damping and frequency in the constantreservoir regime (superscript $\mathrm{cr}$ ):

$$
\begin{aligned}
\Gamma_{\mathrm{RO}}^{\mathrm{cr}, 0} & =\frac{1}{2}\left(\frac{1}{T_{\mathrm{sp}}}+R+2 g S^{*}\right), \\
\omega_{\mathrm{RO}}^{\mathrm{cr}, 0} & =\sqrt{4 g \kappa S^{*}-\Gamma_{\mathrm{RO}}^{\mathrm{cr}, 0^{2}}} .
\end{aligned}
$$

This result is identical to the expressions for the relaxation oscillation parameters obtained from the two-variable system Eq. (3.7), apart from an additional $\frac{R}{2}$ in the relaxation oscillation damping rate. This can be understood by a decrease of the effective carrier lifetime in the quantum-dot states due to the scattering process: The carriers will be driven towards their equilibrium distribution with the effective rate $\left(T_{\mathrm{sp}}{ }^{-1}+R\right)$, which then takes the place of $T_{1}$ in the simple two-variable rate equation system.

The solution of the zeroth-order problem thus predicts an increase in the damping with increasing scattering rate, in accordance with the previous observations. The remaining third eigenvalue $\lambda_{0^{*}}$ is purely real and thus does not contribute to the relaxation oscillations, but instead describes the dynamics of the reservoir, with its lifetime $T_{1}$.

We now proceed in the expansion of the characteristic equation by solving the first-order problem, i.e., taking only terms of $O(d)$ in Eq. (3.30) into account, after inserting Eq. (3.32). The resulting equation reads

$$
\begin{aligned}
0= & 4 g \kappa S^{*} R T_{1} T_{\mathrm{sp}}+\left(R T_{1}+2 g R S^{*} T_{1} T_{\mathrm{sp}}\right) \lambda_{0}+R T_{1} T_{\mathrm{sp}} \lambda_{0}{ }^{2} \\
& +\left[1+R T_{\mathrm{sp}}+2 g S^{*} T_{\mathrm{sp}}+4 g \kappa S^{*} T_{1} T_{\mathrm{sp}}\right. \\
& \left.\quad+2\left(T_{1}+T_{\mathrm{sp}}+R T_{1} T_{\mathrm{sp}}+2 g S^{*} T_{1} T_{\mathrm{sp}}\right) \lambda_{0}+3 T_{1} T_{\mathrm{sp}} \lambda_{0}{ }^{2}\right] \lambda_{1},
\end{aligned}
$$




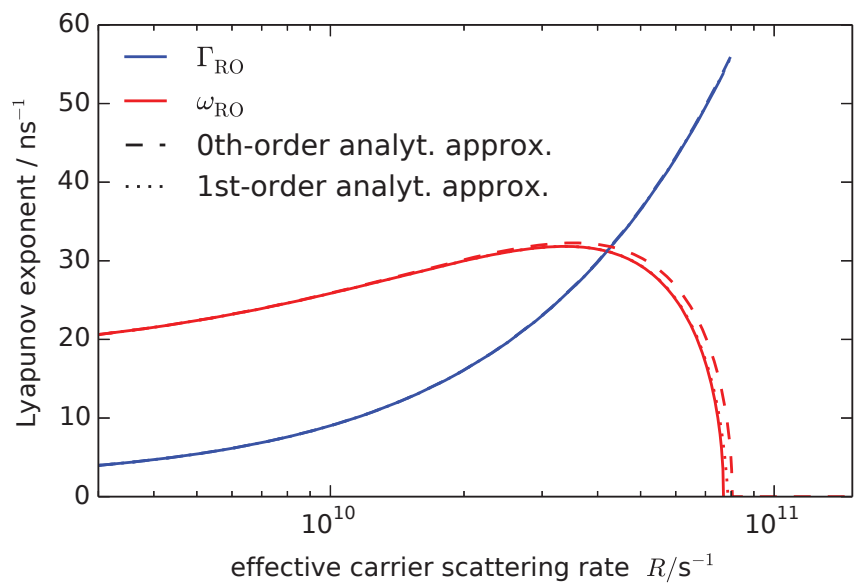

Figure 3.8.: Analytic approximation for the relaxation oscillation (RO) parameters in the constant-reservoir regime. Shown is the RO damping and frequency of the minimal quantum-dot laser model (solid lines), along with the analytic approximation in 0th order (dashed) and 1st order (dotted), cf. Fig. 3.7.

which can be readily solved for $\lambda_{1}$. After additional simplification, the resulting first-order corrections in the constant-reservoir regime then can be written as

$$
\begin{aligned}
& \Gamma_{\mathrm{RO}}^{\mathrm{cr}, 1}=-R^{2} \frac{1}{2\left[\left(\omega_{\mathrm{RO}}^{\mathrm{cr}, 0}\right)^{2}+\left(\Gamma_{\mathrm{RO}}^{\mathrm{cr}, 0}-\frac{1}{T_{1}}\right)^{2}\right]}, \\
& \omega_{\mathrm{RO}}^{\mathrm{cr}, 1}=R^{2} \frac{\left(\Gamma_{\mathrm{RO}}^{\mathrm{cr}, 0}-4 g \kappa S^{*} T_{1}\right)}{2 \omega_{\mathrm{RO}}^{\mathrm{cr}, 0}\left[\frac{1}{T_{1}}-2 \Gamma_{\mathrm{RO}}^{\mathrm{cr}, 0}+4 g \kappa S^{*} T_{1}\right]} .
\end{aligned}
$$

Here we have written $\lambda_{1}$ as

$$
\lambda_{1}=-\Gamma_{\mathrm{RO}}^{\mathrm{cr}, 1} \pm \omega_{\mathrm{RO}}^{\mathrm{cr}, 1},
$$

such that the resulting relaxation oscillation parameters are given by

$$
\begin{aligned}
& \Gamma_{\mathrm{RO}}^{\mathrm{cr}}=\Gamma_{\mathrm{RO}}^{\mathrm{cr}, 0}+d \Gamma_{\mathrm{RO}}^{\mathrm{cr}, 1}+O\left(d^{2}\right), \\
& \omega_{\mathrm{RO}}^{\mathrm{cr}}=\omega_{\mathrm{RO}}^{\mathrm{cr}, 0}+d \omega_{\mathrm{RO}}^{\mathrm{cr}, 1}+O\left(d^{2}\right) .
\end{aligned}
$$

The first-order corrections in $d$ thus introduce an additional quadratic dependence on the scattering rate $R$.

Figure 3.8 shows a comparison of the analytic approximations in zeroth and first order in $d$ with the numerically determined values. It can be seen that both approximations reproduce the dependence of the relaxation oscillation damping 
on the scattering rate very closely. The zeroth order approximation, Eqs. (3.35), shows minor deviations in the frequency at the beginning of the overdamped regime, which are alleviated by the first-order correction terms.

\section{Fast Scattering - Synchronized Regime}

Next, we will look at the synchronized regime for fast charge carrier scattering. In order to simplify the characteristic equation, we evaluate it in the limit of $R \rightarrow \infty$, i.e., taking only terms of $O(R)$ into account. The remaining terms can then be neglected, as the terms including the scattering rate $R$ will dominate the dynamics. The eigenvalues are expanded in a similar manner as before. We choose an expansion with respect to the inverse scattering rate $r:=\frac{1}{R}$ around $r=0$, i.e., $R \rightarrow \infty:$

$$
\lambda=\lambda_{0}+\frac{1}{R} \lambda_{1}+O\left(\frac{1}{R^{2}}\right) .
$$

Inserting this into the characteristic equation Eq. (3.30) and taking only the highest order in $R$ into account, we can write it in the high- $R$ limit as

$$
0=4 d g \kappa S^{*} T_{1} T_{\mathrm{sp}}+\left(d T_{1}+T_{\mathrm{sp}}+2 d g S^{*} T_{1} T_{\mathrm{sp}}\right) \lambda_{0}+(1+d) T_{1} T_{\mathrm{sp}} \lambda_{0}{ }^{2} .
$$

This can be readily solved, and we find the relaxation oscillation damping and frequency in the synchronized regime in zeroth-order approximation:

$$
\begin{aligned}
\Gamma_{\mathrm{RO}}^{\mathrm{s}, 0} & =\frac{1}{2(1+d)}\left(\frac{1}{T_{1}}+\frac{d}{T_{\mathrm{sp}}}+2 d g S^{*}\right), \\
\omega_{\mathrm{RO}}^{\mathrm{s}, 0} & =\sqrt{4 d g \kappa S^{*}-\Gamma_{\mathrm{RO}}^{\mathrm{s}, 0^{2}}} .
\end{aligned}
$$

Similar to the procedure before, this result can now be used to calculate the firstorder correction in $\frac{1}{R}$ to the eigenvalues. We will not explicitly write this down at this point, due to the complexity of the resulting terms (see Appendix A.2).

The analytic approximation is shown together with the numerically determined eigenvalues in Fig. 3.9. The zeroth-order approximation can be seen to reproduce the values in the limit of very high $R$, but fails when reducing the scattering rate, predicting a decrease of the damping. The first-order approximation corrects this and correctly predicts the increase of the damping with decreasing $R$. Nevertheless, the approximation in the synchronized regime is worse as in the constant-reservoir regime. While the damping of the oscillations increases, the first-order approximation cannot predict the onset of the overdamped regime. Here, further orders of the expansion in $R^{-1}$ would have to be taken into account to improve the approximation. However, given the already complex expression for the first-order correction, 


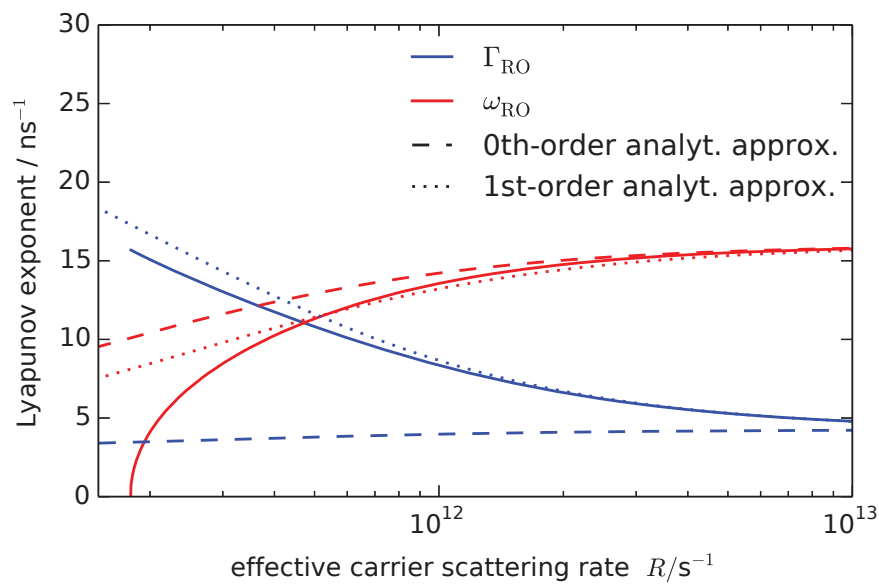

Figure 3.9.: Analytic approximation for the relaxation oscillation (RO) parameters in the synchronized regime, cf. Fig. 3.8.

this approach would likely not produce any practical results. As such, expansions in other variables might yield better results. At this point, however, we will not pursue this problem further, as it would exceed the scope of this work. 


\subsection{Modulation Response of Quantum-Dot Lasers}

High-speed laser devices are a building block of current and future digital data transmission systems [BIM08, CHO11b]. By either direct modulation of the laser output by the pump current or a external modulation of the laser output one can encode digital data streams in optical signals. Quantum-dot lasers have initially been suggested as a replacement for currently used semiconductor lasers due to the prediction of improved characteristics compared to the conventional lasers [BIM97]. However, the maximum attainable modulation speed of quantum-dot lasers still mostly stays behind expectations. This is commonly attributed to the chargecarrier dynamics in quantum-dot lasers [ISH04, SUG05, GIO11, WAN12a, LIN12].

The investigation of the quantum-dot laser response to a small modulation of the pump current can give an indication of the corresponding laser performance. Depending on the

\subsubsection{Small-Signal Response}

The small-signal modulation of the quantum-dot laser quantifies the response of the output power to a small harmonic modulation of the applied pump current. A "small" modulation in this case means that the response of the laser system remains linear, i.e., independent of the modulation amplitude. A quantification of the modulation capabilities of the laser device can be evaluated by the normalized transfer function [COL12a]

$$
H(\omega)=\left|\frac{\Delta P(\omega)}{\Delta P(\omega=0)}\right|^{2}
$$

where $\Delta P(\omega)$ is the modulation amplitude of the output power at a given current modulation frequency $\omega . H(\omega)$ is normalized to the static value $\Delta P(\omega=0)$, which corresponds to the differential output power change at a simple differential change of the pump current.

The transfer function measures the reaction of the output power to perturbations of the driving pump current. This initial perturbation has to "propagate" through the different dynamic variables in the system. The variation in the pump current at first modulates the reservoir carrier-densities, which in turn leads to a modulation of the charge-carrier scattering into the quantum-dot states. The modulated inversion in the quantum-dots then translates into a modulated optical gain, and finally influences the optical power of the laser device. Each of these steps is characterized by a specific timescale determining the speed of the respective coupling process.

Parts of this section have been published in [LIN12]. 
The resulting transfer function will thus be determined by all of these coupling rates, making the experimental analysis of the small-signal modulation behavior of laser devices a tool for a direct measurement of device parameters.

Since the external modulation of the pump current is assumed to be small, the dynamical system will deviate only little from its initial steady state. The analytical treatment of the small-signal modulation can thus be performed by taking into account only the linearized dynamical equations, and neglecting higher order perturbations from the steady-state. Many important aspects of the frequencydependence of the modulation response can already be inferred from the analysis of the simple two-variable rate-equation model. Assuming a harmonically modulated pump current, $J(t)=J^{*}+\Delta J e^{i \omega t}$, with the small modulation amplitude $\Delta J$, the dynamic variables $N, S$ of the dynamic system can be assumed to follow a similar time-dependence:

$$
\begin{aligned}
N(t) & =N^{*}+\Delta N e^{i \omega t} \\
S(t) & =S^{*}+\Delta S e^{i \omega t},
\end{aligned}
$$

with the superscript $*$ denoting their respective steady-state values. Inserting the above expressions into the rate-equation system Eqs. (3.1) then yields:

$$
\begin{aligned}
\frac{\mathrm{d}}{\mathrm{d} t} N(t)=i \omega \Delta N e^{i \omega t}= & J^{*}+\Delta J e^{i \omega t}-2 g\left(N^{*}+\Delta N e^{i \omega t}\right)\left(S^{*}+\Delta S e^{i \omega t}\right) \\
& -\frac{1}{T_{1}}\left(N^{*}+\Delta N e^{i \omega t}\right) \\
\frac{\mathrm{d}}{\mathrm{d} t} S(t)=i \omega \Delta S e^{i \omega t}= & 2 g\left(N^{*}+\Delta N e^{i \omega t}\right)\left(S^{*}+\Delta S e^{i \omega t}\right)-2 \kappa\left(S^{*}+\Delta S e^{i \omega t}\right) .
\end{aligned}
$$

We now neglect the terms with order higher than one in the small amplitudes $\Delta X$ $(X \in\{N, S\})$, thus taking only the first-order deviations from the steady-state into account. Noting that the terms involving only the steady-state values $X^{*}$ on the right hand side of Eqs. (3.45) equal the time-derivatives $\left.\frac{\mathrm{d} X}{\mathrm{~d} t}\right|_{X^{*}}=0$, which vanish in the steady-state, leads to the linearized equations:

$$
\begin{aligned}
& i \omega \Delta N=\Delta J-2 g\left(S^{*} \Delta N+N^{*} \Delta S\right)-\frac{1}{T_{1}} \Delta N \\
& i \omega \Delta S=2 g\left(S^{*} \Delta N+N^{*} \Delta S\right)-2 \kappa \Delta S,
\end{aligned}
$$

or, in matrix form,

$$
(i \omega \underline{\underline{I}}-\underline{\underline{J}})\left(\begin{array}{c}
\Delta N \\
\Delta S
\end{array}\right)=\left(\begin{array}{c}
\Delta J \\
0
\end{array}\right)
$$




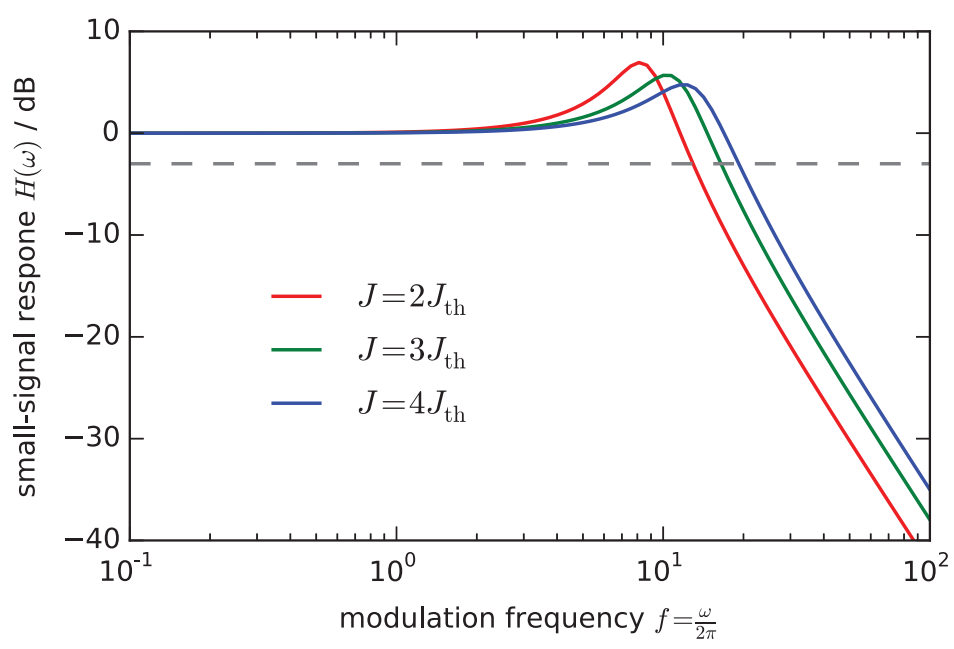

Figure 3.10.: Small-signal modulation transfer function for the two-variable rate-equation system at currents of $2 J_{\text {th }}$ (red), $3 J_{\text {th }}$ (green), and $4 J_{\text {th }}$ (blue). The dashed line denotes a small-signal response of $-3 \mathrm{~dB}$. Parameters as in Fig. 3.1.

with the identity matrix $\underline{\underline{I}}$ and the system Jacobian $\underline{\underline{J}}$. Inverting the above matrix equation leads to the small-signal modulation amplitudes:

$$
\begin{aligned}
\Delta N(\omega) & =\frac{i \omega \Delta J}{4 g \kappa S^{*}-\omega^{2}+i \omega\left(2 g S^{*}+\frac{1}{T_{1}}\right)} \\
\Delta S(\omega) & =\frac{2 g S^{*} \Delta J}{4 g \kappa S^{*}-\omega^{2}+i \omega\left(2 g S^{*}+\frac{1}{T_{1}}\right)} .
\end{aligned}
$$

From the above expression the small-signal modulation transfer function Eq. (3.43) for the simple two-variable rate-equation system can be calculated, under the assumption that the output power is proportional to the photon number $S$ :

$$
H(\omega)=\frac{\omega_{\mathrm{res}}^{4}}{\left(\omega_{\mathrm{res}}^{2}-\omega^{2}\right)^{2}+4 \Gamma_{\mathrm{RO}}^{2} \omega^{2}},
$$

where we have inserted the respective expressions for the relaxation resonance frequency $\omega_{\text {res }}$ and relaxation oscillation damping $\Gamma_{\mathrm{RO}}$. The resulting transfer function is plotted in Fig. 3.10. It shows a resonance peak and a quick decrease afterward for increasing modulation frequency. The peak in the modulation response is caused by the resonant excitation of the relaxation oscillations by the external modulation, greatly enhancing the laser response around $\omega_{\mathrm{RO}}$. For technological applications an important parameter is the $-3 \mathrm{~dB}$ cutoff-frequency, giving the maximum modulation frequency, for which the transfer function is still above $-3 \mathrm{~dB} \approx 0.5$. The 

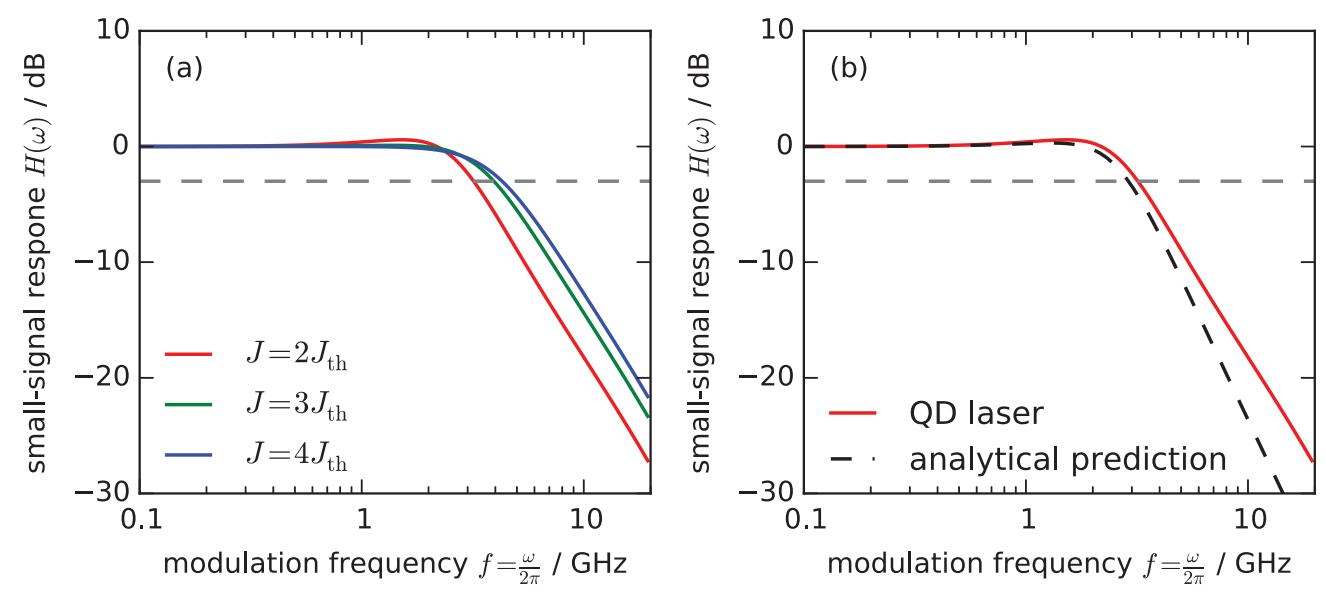

Figure 3.11.: (a) Small-signal modulation response of the quantum-dot laser at pump currents of $2 J_{\text {th }}$ (red), $3 J_{\text {th }}$ (green), $4 J_{\text {th }}$ (blue). (b) Comparison with the analytically predicted transfer function Eq. (3.43) (dashed). The dashed gray line denotes a smallsignal response of $-3 \mathrm{~dB}$.

cutoff-frequency gives an impression of the maximum frequency at which the laser can be modulated.

The small-signal analysis as detailed above can in principle be applied to the given quantum-dot laser model. However, its high dimensionality makes such an analytical treatment impractical. While the small-signal response has been analytically investigated for simpler quantum-dot laser models [ASR10, WAN12a], we evaluate the modulation transfer function by numerical integration. The resulting modulation response curves are shown in Fig. 3.11.

The simulations reveal a relatively flat modulation response, with a shallow and broad resonance peak, which is a direct consequence of the strongly damped relaxation oscillations in the quantum-dot laser. With increasing pump current, the modulation response curves become flatter and the cutoff-frequency increases. This behavior could already be seen in the current dependence of the simple rateequation system and is related to the different scaling of the relaxation oscillation frequency and damping with the output power, leading to less pronounced oscillations with increasing current.

In Fig. $3.11 \mathrm{~b}$ the numerically determined modulation response of the quantumdot laser is compared to the analytical prediction Eq. (3.43), using the relaxation oscillation damping and frequency as parameters, which were obtained from the fits to the quantum-dot laser response to a small perturbation. The analytical 


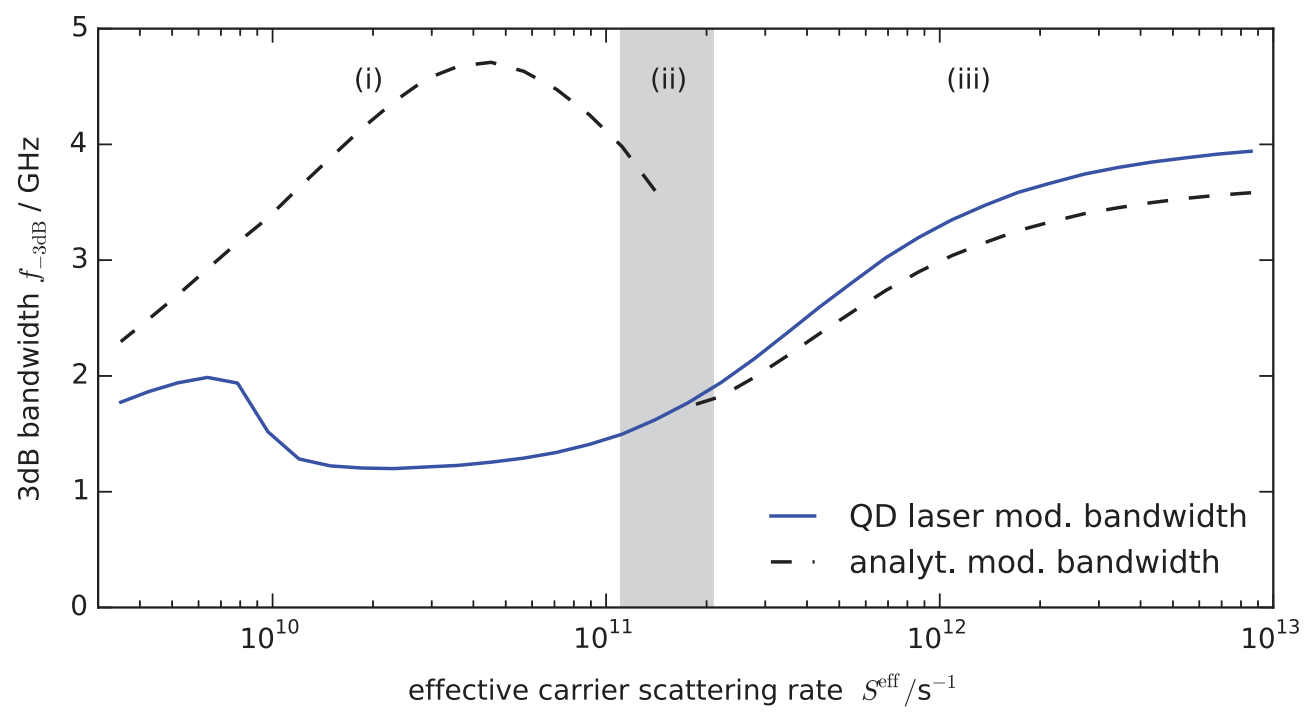

Figure 3.12.: Small-signal modulation bandwidth of the quantum-dot laser. Shown is the modulation frequency $f_{-3 \mathrm{~dB}}$ at which the modulation response reaches $-3 \mathrm{~dB} \approx \frac{1}{2}$ for the first time (blue solid curve). Also shown is the modulation bandwidth predicted from Eq. (3.43) (black dashed). The pump current was set to twice the respective threshold current at each data point. The three dynamic regions are as noted in Fig. 3.6. After [LIN12].

formula is found to match the quantum-dot response closely, leading to a good approximation of the modulation bandwidth for the given parameters.

As we have seen previously in Fig. 3.1, the relaxation oscillations in quantumdot lasers are strongly dependent on the scattering timescales. We will therefore look at the modulation capabilities of the quantum-dot laser for different scattering times. The resulting modulation bandwidths in dependence of the effective carrier scattering rate is shown in Fig. 3.12. The bandwidth can be seen to increase with the effective scattering rate in the overdamped and synchronized dynamic regimes. This is in line with the common assumption of a carrier "bottleneck" between the reservoir and the quantum-dot states limiting the modulation response [HEI01, TON09]. However, when comparing the modulation bandwidth of the quantum-dot laser within the synchronized regime with the analytically predicted bandwidth using Eq. (3.43), it becomes evident that the quantum-dot laser exhibits modulation capabilities up to higher frequencies than predicted by the simple rate-equation approach.

The constant-reservoir regime reveals a non-monotonous behavior, with a relatively sharp increase in the bandwidth for scattering rates below $\approx 10^{10} \mathrm{~s}^{-1}$. When 
comparing the numerical results and analytical predictions, it becomes clear that in this dynamic regime the simple two-variable approach fails. Using the numerically determined relaxation oscillation damping and frequency as input parameters, Eq. (3.43) predicts a much higher modulation bandwidth than we observe in the simulations. The reason for this is the strong dynamical interaction between the resonant quantum-dot charge carriers and the reservoir, which is not accounted for in the analytical approximation. For the limit of very slow scattering in the constant-reservoir regime, the sudden increase in the modulation bandwidth means that the numerical and analytical curves start converging. This can be understood by the weak interaction between the resonant and off-resonant states. Since the reservoir is nearly unaffected by the relaxation oscillations, they provide a constant carrier influx into the quantum-dot states. This situation is then akin to the simple two-variable system, where a constant pump current into the optically active states is assumed, and the modulation response is well described by the analytical approximation. However, it must be noted that in this regime the operating current must be much higher to provide a sufficiently high in-scattering of charge carriers into the quantum-dots to enable the lasing action. This makes practical applications improbable, as also the absolute modulation response of the quantum-dot laser is strongly inhibited, i.e., a very high modulation amplitude would have to be applied to achieve appreciable modulation of the output power.

A detailed plot of the modulation response curves for different scattering rates is shown in Fig. 3.13. For fast scattering (dark red in Fig. 3.13), the transfer function reveals the already known behavior, with a single resonance peak, after which the modulation response drops steadily. With decreasing scattering rates, the resonance peak disappears, due to the increasing damping and subsequent disappearance of the relaxation oscillations (yellow to green), which decreases the modulation bandwidth. For even slower scattering, again a peak appears which, however, only for very slow scattering becomes appreciably large, leading to the sudden increase in modulation bandwidth for $S^{\text {eff }} \lesssim 10^{10} \mathrm{~s}^{-1}$. In the range $10^{10} \mathrm{~s}^{-1} \lesssim S^{\text {eff }} \lesssim 10^{11} \mathrm{~s}^{-1}$, where the analytical approximation would predict a much higher modulation bandwidth, the interaction between the quantum-dot and reservoir states is still strong enough to suppress the modulation. 


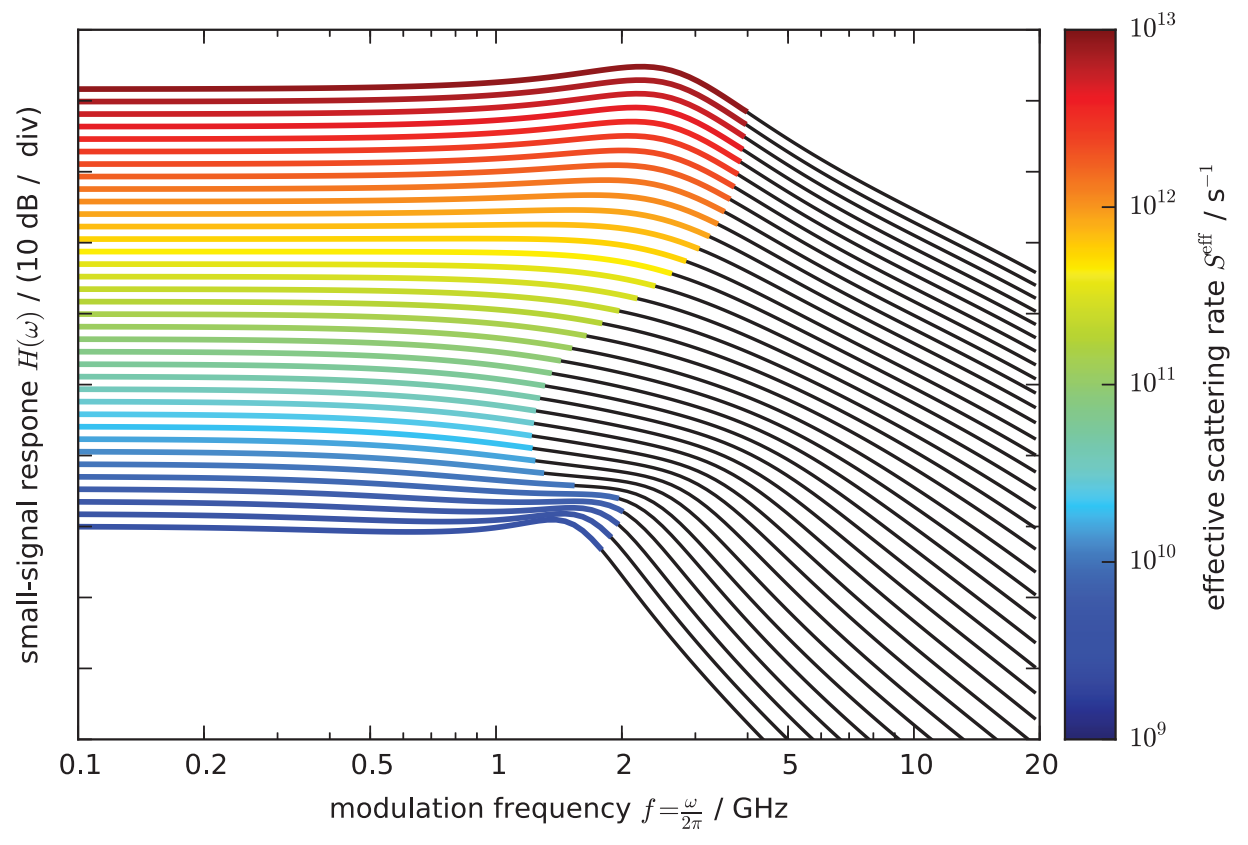

Figure 3.13.: Dependence of the quantum-dot laser modulation response on the effective scattering rate $S^{\text {eff }}$. Shown are the small-signal modulation response curves for different values of $S^{\text {eff }}$, given by the color-code. The curves have been shifted along the vertical axis for better readability. The black parts of the curves denote the parts for which the response is below $-3 \mathrm{~dB}$. 


\subsection{Amplitude-Phase Coupling in Quantum-Dot Lasers}

As we have seen previously, the light-matter interaction in the active region between the optical field and the semiconductor transitions leads to either gain or absorption, as well as a change in the effective refractive index. These two contributions can be expressed by the complex gain $g(\omega, t)$ for a given lasing frequency $\omega$, and are coupled by the dynamics of the charge carriers in the different optically active states. This interplay of gain and refractive index (or frequency shift) is commonly referred to as amplitude-phase coupling.

In semiconductor lasers, the connection between the refractive index and the optical gain plays an important role in many applications and phenomena. The response to external modulations or perturbations [RAD07, GRI11], the laser linewidth [HEN82], and the occurrence of dynamical instabilities as well as pattern formation in spatially extended laser systems [SMO02, RIB03] all crucially depend on this amplitude-phase coupling. Throughout the literature, this connection is commonly described by assuming a linear relationship between changes of the resonance frequency shift and gain. The proportionality factor is called the linewidthenhancement factor $\alpha$.

This $\alpha$-factor is commonly described as a constant device parameter, and used to characterize the dynamical performance of the laser device. In general, a high value of $\alpha$ is related to highly complex dynamics, unstable laser operation, and a large laser linewidth. For example, in optical injection [WIE05, LIN13] or optical feedback setups [ОTT10, LIN12b] the resulting bifurcation structure becomes largely more complex with increasing alpha. Furthermore, the minimum feedback strength required to introduce dynamical instabilities or chaos is lowered by $\alpha$ [LEV95, ERN10b, OTT14], which makes a diligent suppression of undesired reflections in optical setups necessary.

On the other hand, there are applications which rely on the existence of complex dynamic solutions. For example, chaotic semiconductor lasers have been successfully used for random-number generation [REI09a, OLI11, HAR12, NGU12a], as well as for secure communication [TRO08, LUE11b, UCH12]. Furthermore, their complex dynamics allow for the studying of many generic nonlinear phenomena in a comparably simple experimental setup [LAR10]. For example, the appearance of chimera states - coexisting coherent and incoherent states in coupled dynamic systems - has been recently predicted in coupled laser networks, with the amplitude-phase coupling as a driving force [BOE15].

In the following, we will investigate the amplitude-phase coupling in quantumdot lasers. In particular, we will look at the dynamics of the electric field phase

Parts of this section have been published in [LIN13]. 
induced by the refractive-index dynamics in optical injection and feedback setups, as well as under direct modulation. We will furthermore discuss the applicability and limitations of the $\alpha$-factor for quantum-dot lasers, by comparing our microscopically based modeling approach with conventional, simpler, approaches.

\subsubsection{The Linewidth-Enhancement Factor $\alpha$}

Under the dynamic operation of lasers, the complex optical gain changes with the inversion of all optically active transitions in the active medium. Following Eq. (2.75), the complex optical gain inside the laser cavity can generally be written as

$$
g(\omega, t)=\frac{\hbar \omega}{\varepsilon_{0} \varepsilon_{\mathrm{bg}} V} \sum_{i} g^{i}(\omega)\left[\rho_{e}^{i}(t)+\rho_{h}^{i}(t)-1\right],
$$

with the contribution of the individual transitions labeled by the index $i$, which can include quantum-dot and reservoir transitions,

$$
g^{i}(\omega)=\frac{T_{2}\left|\mu^{i}\right|^{2}}{2 \hbar^{2}}\left(\frac{1-i T_{2}\left(\omega^{i}-\omega\right)}{1+\left[T_{2}\left(\omega^{i}-\omega\right)\right]^{2}}\right) .
$$

As we have shown earlier, the charge-carrier-induced change of the refractive index $\Delta n$, or, analogously, the shift of the resonance frequency $\Delta \omega$, can be written as

$$
\Delta \omega=-\omega \frac{\Delta n}{n_{\mathrm{bg}}}=-\operatorname{Im} g(\omega, t),
$$

with the background index $n_{\text {bg }}$ and the cold-cavity lasing frequency $\omega$. As the imaginary part of the complex gain is determined from the off-resonant optical transitions, the refractive index dynamics is also determined by these states, whereas the amplitude gain is determined from the resonant transitions.

As the different states in semiconductor lasers are coupled, also the dynamic changes of the optical gain and refractive index will be coupled. Henry first explained the high linewidth of semiconductor lasers by introducing the linewidthenhancement (or Henry-) factor $\alpha$, as the ratio of the real and imaginary part of the variation of the optical susceptibility with the charge carrier number $N$ :

$$
\alpha:=\frac{\partial \chi^{\prime} / \partial N}{\partial \chi^{\prime \prime} / \partial N},
$$

or, written in terms of the complex gain $g$ :

$$
\alpha=-\frac{\partial \operatorname{Im} g / \partial N}{\partial \operatorname{Re} g / \partial N}
$$


The mechanism responsible for the linewidth broadening then is the following:

By spontaneous emission processes, both the charge carrier distribution as well as the optical field are subjected to statistical fluctuations. Due to the random phase of spontaneously emitted photons, the phase of the optical field exhibits a random walk, which limits the coherence time $\tau_{\text {coh }}$ of the laser output. The WienerKhinchin-theorem [WIE30, GAR06] then states that the spectral linewidth $\Delta \nu$ of the laser output is related to the coherence time via $\Delta \nu=\left(\pi \tau_{\text {coh }}\right)^{-1}$. The resulting laser linewidth then leads to the famous Schawlow-Townes linewidth $\Delta \nu^{\mathrm{ST}}$ [SCH58]. Experimental results, however, found a much larger linewidth than predicted [FLE81].

In order to explain this, not only the direct contribution of the spontaneously emitted light to the linewidth must be taken into account. Additionally, the fluctuations in the light intensity will translate into a fluctuating charge carrier distribution by the change in the stimulated emission rate. Thus, variations in the gain and refractive index will arise, which additionally influences the optical field phase. This fluctuation of the phase then leads to an additional shortening of the coherence time, and consequently to a broadening of the laser linewidth, which is given by [LAX67, HEN82]

$$
\Delta \nu=\left(1+\alpha^{2}\right) \Delta \nu^{\mathrm{ST}} .
$$

The concept of the $\alpha$-factor has since been employed for characterizing laser devices, as it provides a simple and experimentally accessible way of describing the connection between index and gain changes in the device [TOF92]. Following its definition Eq. (3.54), the $\alpha$-factor can be used to write the dynamic equation for the electric field inside the laser cavity in a simple way:

$$
\frac{\mathrm{d}}{\mathrm{d} t} E(t)=(1-i \alpha) \operatorname{Re}[g(\omega, t)] E(t)-\kappa E(t),
$$

thus implementing the linear relation between the instantaneous frequency shift, $-\operatorname{Im} g(\omega, t)$, and the amplitude gain $\operatorname{Re} g(\omega, t)$. This assumption is, of course, generally not fulfilled, as the dependence of the two components on the charge carrier number can be nonlinear, and depend on the density of states of the active medium, as sketched in Fig. 3.14. One could, however, argue that above threshold, where most lasers will be operated, the optical gain is clamped to its threshold value, and thus deviations from the value of $\alpha$ at threshold will be small. This is a commonly made assumption that works well in bulk and quantum-well lasers. In fact, this approach is widely used in modeling approaches for conventional semiconductor lasers, and has been successfully used to describe a multitude of dynamical scenar- 


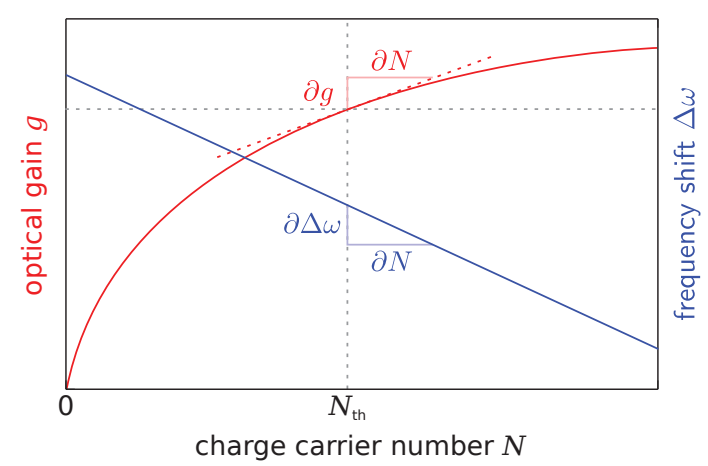

Figure 3.14.: Sketch of the $\alpha$-factor in conventional laser models. The dependencies of the gain and frequency shift on the charge-carrier number are linearized around their value at the threshold carrier number $N_{\text {th }}$. The ratio of their slopes then define the $\alpha$-factor.

ios [ERN10b, LUE11b, OTT14]. We will, however, show that in quantum-dot lasers this assumption is no longer valid, and the concept of $\alpha$ breaks down.

\subsubsection{Charge-Carrier-Induced Susceptibility in Quantum-Dot Lasers}

As we have stressed in the previous chapter, the macroscopic dynamics of quantumdot laser devices is crucially influenced by the microscopic charge-carrier dynamics within the active semiconductor medium. We have shown that the charge-carrier scattering between the quantum-dot and surrounding reservoir states can generate important difference in the dynamic response of quantum-dot lasers compared to conventional bulk semiconductor or quantum-well laser systems. We now investigate the required modifications to the concept of the $\alpha$-factor, which are needed to account for the different behavior of quantum-dot lasers.

After the advent of quantum-dot lasers, the well-established $\alpha$-factor approach has been widely also applied to this new type of laser device, and most works still rely on this description [HUY04, GRI08a, KIM10e, KEL11]. When semiconductor quantum-dots were first proposed, initial theoretical works suggested that the amplitude-phase coupling in these novel devices should be approximately zero, based on the atom-like energy states of quantum-dots [ASA86]. Atom lasers operating at their optical resonance do, in fact, exhibit $\alpha=0$. This becomes immediately clear from Eq. (3.51), where for vanishing detuning of the lasing frequency from the atom resonance the imaginary part of the gain is zero. Early studies, however, already showed that the influence of off-resonant optical transitions of the quantum-dot excited states and reservoir states lead to non-vanishing index variations [BIM97a]. 
Furthermore, there have been theoretical and experimental indications that the use of an $\alpha$-factor in quantum-dot lasers can be inaccurate. While in quantumwell lasers values of $\alpha \approx 2 \ldots 5$ are commonly measured, the measured $\alpha$-factors in quantum-dot lasers range from near-zero [NEW99a, KON04a, ALE07] to very high values [DAG05], and even "infinite" $\alpha$ [CON07, GRI08a]. Furthermore, a strong dependence on the pump current has been observed [SU05a, JIA12]. Theoretical works have shown that the $\alpha$-factor measurements in quantum-dot lasers will yield different results depending on the measurement procedure and operating parameters [MEL06, GIO07, LIN12a].

The reasons for this unconventional behavior lie in the charge carrier dynamics of the quantum-dot material. In its original definition, Henry introduced the alpha factor using the derivatives of the optical susceptibility (or gain) with respect to the total charge carrier number or density. This derivative, however, is only welldefined if there exists a functional dependence of the gain and index on the charge carrier number. In bulk and quantum-well material systems, this requirement is approximately fulfilled due to the fast quasi-equilibration of the charge carrier distribution, provided the optical power is not exceedingly high [AGR93a]. This allows one to write the charge carrier distribution for each individual state $i$ as a function of the total charge carrier number:

$$
\rho_{m}^{i}=f\left(\varepsilon_{m}^{i}, E_{\mathrm{F}, b}^{\mathrm{eq}}, T^{\mathrm{eq}}\right) \equiv \rho_{m}^{i}(N),
$$

with the quasi-Fermi distribution $f$, in which the quasi-Fermi energy $E_{\mathrm{F}, b}^{\mathrm{eq}}$ is a unique function of the carrier number $N$.

Thus, the $\alpha$-factor can be written as a sum over the individual transitions:

$$
\begin{aligned}
\alpha & =-\frac{\partial \operatorname{Im} g / \partial N}{\partial \operatorname{Re} g / \partial N} \\
& =-\left[\sum_{i, b} \frac{\partial \operatorname{Im} g}{\partial \rho_{b}^{i}} \frac{\partial \rho_{b}^{i}}{\partial N}\right] /\left[\sum_{i, b} \frac{\partial \operatorname{Re} g}{\partial \rho_{b}^{i}} \frac{\partial \rho_{b}^{i}}{\partial N}\right] \\
& =-\left[\sum_{i, b} \operatorname{Im} g^{i} \frac{\partial \rho_{b}^{i}}{\partial N}\right] /\left[\sum_{i, b} \operatorname{Re} g^{i} \frac{\partial \rho_{b}^{i}}{\partial N}\right] .
\end{aligned}
$$

During operation of the laser device, the change in charge carrier number $N$ can be caused by different means. Variations in the pump current, the effective optical loss rate, as well as the dynamical change in the carrier number, e.g. during relaxation oscillations, will lead to variations in $N$. 
In quantum-dot lasers, the above expression for the $\alpha$-factor can be written as the sum over all contributions to the gain from the individual optical transitions,

$$
\alpha=-\frac{\left[2 N^{\mathrm{QD}} \sum_{m, b, j} f(j) \nu_{m} \operatorname{Im} g_{m}^{j} \delta \rho_{b, m}^{j}+\frac{2}{A_{\mathrm{act}}} \sum_{b, \boldsymbol{k}^{2 D}} \operatorname{Im} g_{\boldsymbol{k}}^{2 \mathrm{D}} \delta \rho_{b, \mathrm{QW}}(\boldsymbol{k})\right]}{\left[2 N^{\mathrm{QD}} \sum_{m, b, j} f(j) \nu_{m} \operatorname{Re} g_{m}^{j} \delta \rho_{b, m}^{j}+\frac{2}{A_{\mathrm{act}}} \sum_{b, \boldsymbol{k}^{2 D}} \operatorname{Re} g_{\boldsymbol{k}}^{2 \mathrm{D}} \delta \rho_{b, \mathrm{QW}}(\boldsymbol{k})\right]} .
$$

Here, $\delta \rho$ describe the variation of the charge-carrier distribution of the corresponding states. As we have stressed before, the charge-carrier distribution in quantumdot lasers can deviate appreciably from a quasi-equilibrium distribution due to the complex scattering mechanisms involved in the charge-carrier dynamics. Therefore, it is no longer possible to define a functional relationship between the occupation of individual charge-carrier states and the total charge-carrier number. The definition of derivatives $\partial \rho / \partial N$ is therefore in general not possible. Instead, we have to express the changes of the individual occupations as general variations $\delta \rho$.

The evaluation of Eq. (3.59) is thus not as straightforward as in conventional laser devices. Whereas the definition Eq. (3.54) involves the evaluation of the derivatives at a given operation point, in quantum-dot lasers Eq. (3.59) requires a perturbing event to induce the variation of the charge-carrier distribution. The exact shape of this variation is initially unknown and depends on the exact source of the perturbation.

In order to illustrate this, we simulate the reaction of the quantum-dot laser towards external perturbations. Here we consider two different types of perturbation sources: excitation by a fast change in the pump current, and the injection of an external optical pulse resonant to the ground-state transition into the laser cavity. In both cases, we operate the laser at twice the threshold current and apply the perturbation as Gaussian-shaped pulses. We therefore write the pump current as

$$
J(t)=J_{0}+\Delta J \exp \left[-4 \log 2\left(\frac{t-t_{0}}{\Delta t}\right)^{2}\right]
$$

with the pulse amplitude $\Delta J$ and the pulse FWHM $\delta t$. Analogously, we write the optical perturbation pulse as an additional contribution to the electric field dynamic equation:

$$
\left.\frac{\partial}{\partial t} E(t)\right|_{\text {inj }}=K \kappa E^{0} \exp \left[-4 \log 2\left(\frac{t-t_{0}}{\Delta t}\right)^{2}\right],
$$



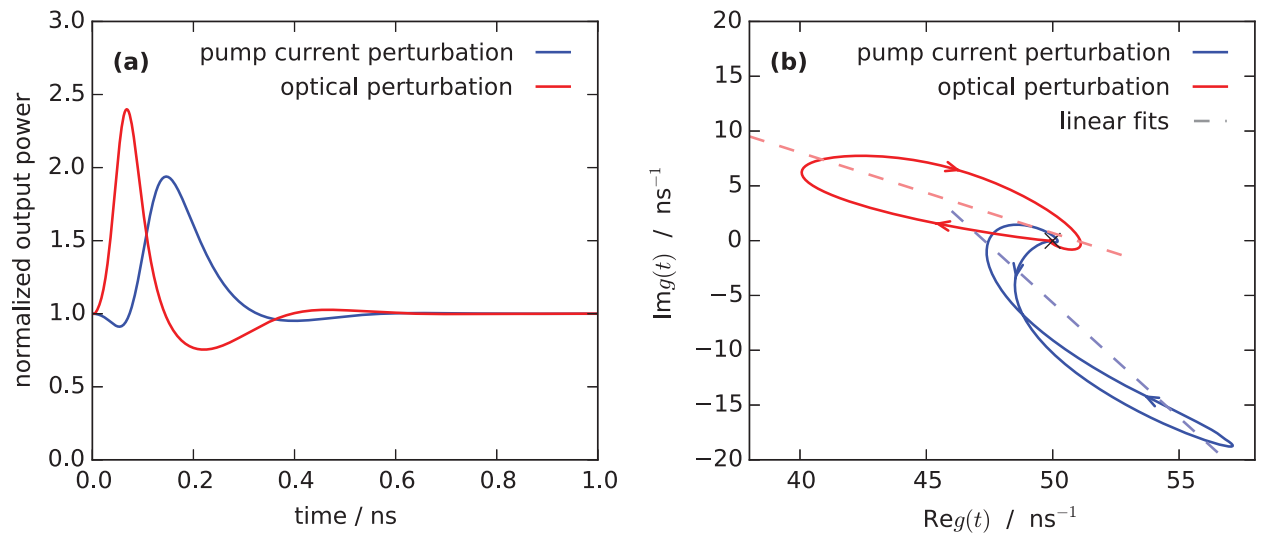

Figure 3.15.: Reaction of the quantum-dot laser to optical and pump-current perturbations. (a) Shown is the output power after an initial perturbation pulse in the pump current (blue) and by an external optical pulse (red). The perturbations are Gaussianshaped with an FWHM of $50 \mathrm{ps}$ and centered around $t=50 \mathrm{ps}$. (b) Phase-space plot in the plane spanned by the complex gain. The stable fixed point is marked by the cross. The trajectories show the response of the gain to the perturbation pulses. The dashed lines show linear fits to the simulated trajectory curves.

where the constant $E^{0}$ denotes the electric field amplitude in the steady-state, $\kappa$ is the optical loss rate, and $K$ then defines a dimensionless injection strength. The choice of the prefactor $K \kappa E^{0}$ is arbitrary and we will motivate it later on.

The reaction to the perturbations is plotted in Fig. 3.15. Here, we chose $\Delta J=$ $J_{\mathrm{th}}, \Delta t=50 \mathrm{ps}, t_{0}=50 \mathrm{ps}, K=0.5$. The time-series shown in Fig. 3.15 a reveals an increase in the output power as a response to both perturbation schemes, with subsequent relaxation oscillations as the laser is driven back into its fixed point. Fig. $3.15 \mathrm{~b}$ shows the trajectories in the complex plane spanned by the optical gain $(\operatorname{Re} g(t), \operatorname{Im} g(t))$. These exhibit clearly quite complex forms, which differ qualitatively between the two cases.

The importance of these trajectories lies in the definition of the $\alpha$-factor. From Eq. (3.54) it becomes evident that $\alpha$ describes the slope of the trajectories shown in Fig. 3.15 b. The conventional way of using $\alpha$ - as a constant - would imply a linear relationship between the real and imaginary parts of $g(t)$, from which the quantumdot laser clearly deviates. A possible approximation of the gain dynamics would be a linear fit to the shown trajectories, yielding the average slope of the trajectories as an averaged $\alpha$-factor, as shown by the dashed lines. Even then, however, the averaged values calculate to $\left\langle\alpha_{J}\right\rangle \approx 2.1$ for the pump-current perturbation and $\left\langle\alpha_{E}\right\rangle \approx 0.73$ with the injected optical pulse. Thus, even around the same fixed point, no uniform value of an average $\alpha$ can be defined [GIO07, LIN12b]. The $\alpha$ - 

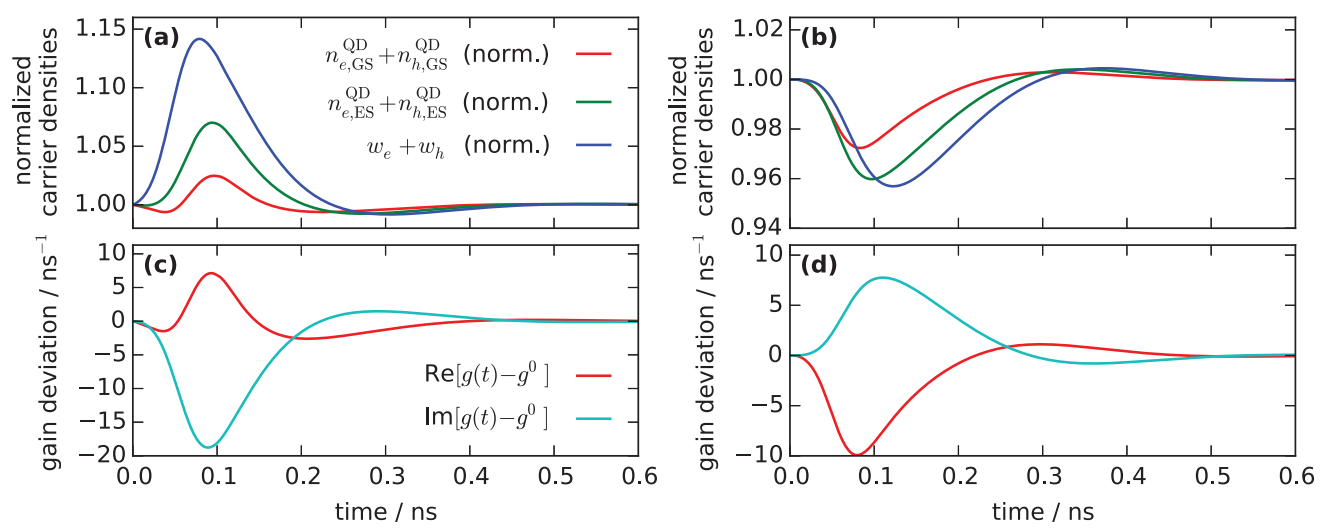

Figure 3.16.: Charge-carrier densities of the quantum-dot laser under (a) pump-current and (b) optical perturbations. Shown are the total quantum-dot ground-state chargecarrier densities (red), the excited-state densities (green), and the reservoir densities (blue). (c),(d) The corresponding real (red) and imaginary part (cyan) of the complex gain after the perturbation, relative to the steady-state gain $g^{0}$.

factor is therefore an inappropriate measure for describing the gain dynamics of the quantum-dot medium.

The reason for the unconventional behavior of the quantum-dot laser can be seen in the charge-carrier dynamics after the perturbation. This is shown in Fig. 3.16 a. The pump current pulse induces a large increase in the reservoir charge-carriers, which initially leads to a slight decrease of the quantum-dot occupations due to carrier-heating effects. The charge-carrier buildup is transmitted to the quantumdot states in a delayed fashion, due to the finite charge-carrier scattering rates. Thus, the excited-state quantum-dot carriers are first affected by the perturbation, and quickly after that the ground-state. The subsequent relaxation oscillations also exhibit such desynchronized dynamics between the different charge-carrier subensembles. As the different charge carriers contribute to the real and imaginary parts of the total gain to different extents, this behavior translates into the desynchronized variation of the optical gain and carrier-induced frequency shift, shown in Fig. 3.16 c. The complex behavior of the trajectories in the complex gain plane is thus a direct consequence of the underlying charge-carrier dynamics. When considering the optical perturbation, this becomes even more apparent. The carrier dynamics shown in Fig. 3.16 b reveal a strong initial depletion of the quantum-dot ground-state carriers due to the increased stimulated recombination rate by the injected optical field. This perturbation is then slowly transmitted to the excitedstate and reservoir charge carriers, with a large time-lag between the individual densities, again leading to strongly desynchronized dynamics. Compared to the 
case we have considered before, now the quantum-dot ground-state assumes the "leading" role, with the changes in the excited state and reservoir lagging behind. This observation reveals the importance of the origin of the charge-carrier variation in the quantum-dot laser, as the dynamics very clearly differ depending on the type of perturbation.

In the following, we will thus perform simulations of the quantum-dot laser under different dynamical setups, in order to highlight the differences in the predicted laser dynamics resulting from the more complex amplitude-phase coupling in quantum-dot lasers. 


\subsection{Dynamics under Optical Injection}

In this section we will discuss the dynamics of quantum-dot lasers under optical injection. Optical injection describes the operation of the laser device under the injection of an external optical signal into the laser cavity, as sketched in Fig. 3.17. Depending on the properties of the injected signal, the laser can exhibit versatile dynamics.

Often, a "master" laser is used to inject a quasi-monochromatic beam into the cavity of the so-called "slave" laser. If the frequencies of both lasers are sufficiently close, the slave laser will be phase-locked to the injected signal, a phenomenon known from driven oscillators [ADL73]. This mechanism was quickly found to be of technological importance, as the properties of the slave laser can be changed by phase-locking to an appropriate master signal, allowing for a sensitive tuning of the lasing frequency to that of the master laser. Additionally, a considerable reduction of the laser noise and linewidth by injection of a very stable, monochromatic signal is possible [ERI71]. For this application, the power of the master laser can be much lower than that of the slave laser, allowing for the realization of high-power laser devices with extremely low linewidth [LIU02a]. Furthermore, the modulation bandwidth of laser devices was shown to be greatly increased by an appropriate injection setup [JIN06, TER08a, LAU09a], with bandwidths exceeding $100 \mathrm{GHz}$ [LAU08a].

The nonlinear dynamics of semiconductor lasers under external optical perturbations have been subject of extensive experimental and theoretical studies in the past [WIE05, NAD09, KEL11a]. When the frequency of the injected signal is too large, the beating between the external and cavity fields can induce nonlinear oscillations [TRE85] as well as deterministic chaos [SIM94a], multi-stability [GAV97], or excitability close to the boundary of phase-locking [GOU07]. Apart from their scientific appeal, these nonlinear dynamics can also be exploited and used for technological applications. For example, optically injected lasers have been implemented as an all-optical memory or switch, allowing for a toggling between two different stable states by external trigger signals [OSB09a, OSB12]. Furthermore, optically injected

Parts of this section have been published in [LIN12b, LIN13].

Figure 3.17: Sketch of the optical injection scheme. The external optical signal $E_{\text {inj }}$ has a frequency detuning $\Delta \omega_{\text {inj }}$ with respect to the free-running laser. The optical interaction within the laser cavity can lead to dynamic solutions.

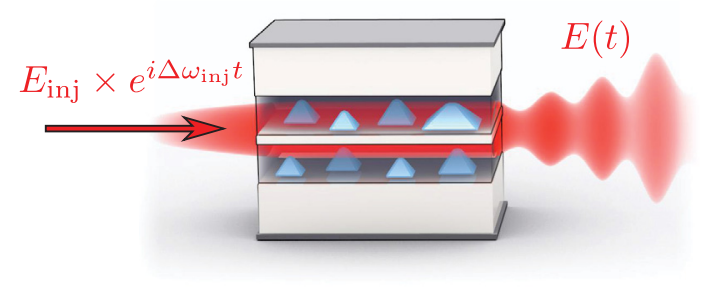


quantum-dot lasers have been shown to be a promising source for the generation of THz-radiation [HUR13].

In the following, we will analyze the nonlinear dynamics of quantum-dot lasers under optical injection. We will focus on the influences of the carrier dynamics in quantum-dot lasers and the differences in the response of the laser to optical injection compared to conventional laser devices.

\subsubsection{Quantum-Dot Laser Model with Optical Injection}

In order to take into account the effect of optical injection on the quantum-dot laser we have to expand our model. The injected signal will enter the electric field equation as an additional driving term, which we will derive here.

Considering an external optical field $E_{\text {inj }}$ present at the outside of the cavity mirror, we can calculate the portion of this field transmitted into the laser cavity as $t \cdot E_{\mathrm{inj}}$, with the cavity mirror transmission coefficient $t$. After half of the cavityround-trip time $\tau_{\text {in }}$ the injected signal arrives at the back facet, where it is reflected with a reflection coefficient $r$. The portion $r t E_{\text {inj }}$ arrives again at the front facet after another half round-trip time, as illustrated in Fig. 3.18. From this simple consideration, we can write the change of the average electric field amplitude in the laser cavity after one round-trip time due to the injected signal as [WIE05]

$$
\left[E\left(t+\tau_{\text {in }}\right)-E(t)\right]_{\text {inj }}=\frac{1}{2} t E_{\text {inj }}+\frac{1}{2} r t E_{\text {inj }} .
$$

Considering now that the internal round-trip time is usually smaller than the dominant dynamic time-scales within the laser, i.e., the relaxation oscillations, we can approximate the above equation as a derivative:

$$
\left.\frac{\partial}{\partial t} E(t)\right|_{\mathrm{inj}}=\frac{t(r+1)}{2 \tau_{\mathrm{in}}} E_{\mathrm{inj}} \equiv k_{\mathrm{inj}} E_{\mathrm{inj}}
$$

where we have summarized the prefactors into the injection rate $k_{\mathrm{inj}}$. The dynamic equation for the electric field is thus written as

$$
\frac{\mathrm{d}}{\mathrm{d} t} E(t)=(g(t)-\kappa) E+k_{\mathrm{inj}} E_{\mathrm{inj}}
$$

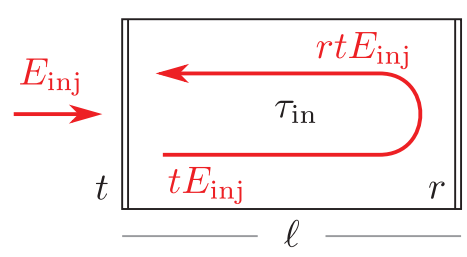

Figure 3.18: Optical injection into the laser cavity. The external optical field $E_{\text {inj }}$ is transmitted through the cavity mirror with transmission coefficient $t$, and reflected at the back-facet (reflection coefficient $r$ ). After the internal round-trip time $\tau_{\text {in }}=\frac{n \ell}{c}$ the injected signal has filled the whole cavity. 
We now introduce the relative injection strength $K$, defined via

$$
K \kappa E^{0} \equiv k_{\text {inj }} E_{\text {inj }},
$$

where $E^{0}$ is the steady-state electric field amplitude of the free-running laser. The term $\kappa E^{0}$ thus describes the electric field amplitude the free-running laser loses per time interval. $K$ then describes the electric field injected into the cavity in relation to the field that is lost.

We write the injected signal as

$$
E_{\mathrm{inj}}(t)=\left|E_{\mathrm{inj}}\right| e^{-i \omega_{\mathrm{inj}} t},
$$

where $\omega_{\text {inj }}$ is the optical frequency of the injected signal in the rotating reference frame of the chosen carrier frequency $\omega$. As we have stressed before, the charge carriers in the laser active medium will lead to a change in refractive index, which in turn shifts the laser resonance frequency away from the rotating frame frequency. Thus, in the lasing steady-state, the free-running laser will operate on a frequency given by

$$
\widetilde{\omega}^{0}=\omega-\operatorname{Im} g^{0} \equiv \omega+\omega^{0},
$$

where $g^{0}$ is the complex optical gain evaluated in the lasing steady-state of the free-running laser and $\omega^{0}$ the corresponding induced frequency shift. Note that a positive value of $\omega^{0}$, i.e., a frequency increase, corresponds to a mathematical negative sense of rotation of $E(t)$. Within the rotating frame the optical field in the steady state of the free-running laser is thus given by

$$
\left.E(t)\right|_{\mathrm{fr}}=E^{0} e^{-i \omega^{0} t}
$$

We now consider a possible detuning between the injected signal and the optical frequency of the free-running laser. We thus write $\omega_{\text {inj }}=\omega^{0}+2 \pi \Delta \nu_{\text {inj }}$. The electric field equation is thus written as

$$
\frac{\mathrm{d}}{\mathrm{d} t} E(t)=(g(t)-\kappa) E+K \kappa E^{0} e^{-i\left(\omega^{0}+2 \pi \Delta \nu_{\mathrm{inj}}\right) t} .
$$

This choice of $\Delta \nu_{\text {inj }}$ means that a positive value of the detuning refers to an optical frequency of the master laser that is higher compared to the slave laser.

The explicit time-dependence of the injection term in the above equation results in a system that is non-autonomous. We can, however, write it in an autonomous form by a change of the rotating frame. We introduce the new electric field variable

$$
\widetilde{E}(t):=E(t) e^{i\left(\omega^{0}+2 \pi \Delta \nu_{\mathrm{inj}}\right) t},
$$


which transforms the electric field $E(t)$ in the rotating frame of the carrier frequency $\omega$ into a new rotating frame of the frequency $\left(\omega+\omega^{0}+2 \pi \Delta \nu_{\text {inj }}\right)=\left(\omega+\omega_{\text {inj }}\right)$, which is the optical frequency of the injected signal. Rewriting the electric field equation in this new rotating frame, we arrive at

$$
\frac{\mathrm{d}}{\mathrm{d} t} \widetilde{E}(t)=\left(g(t)-\kappa+i \omega^{0}\right) \widetilde{E}+i 2 \pi \Delta \nu_{\mathrm{inj}} \widetilde{E}+K \kappa E^{0} .
$$

The first of the newly added terms, $i \omega^{0} \widetilde{E}$, compensates the carrier-induced frequency shift in the free-running case. Thus, without injection, i.e. $K=\Delta \nu_{\text {inj }}=0$, when the laser reaches its steady-state, $\frac{\mathrm{d}}{\mathrm{d} t} \widetilde{E}$ vanishes, since $i \omega^{0}=-\operatorname{Im} g^{0}$. This was not the case in the old rotating frame, as there the electric field would rotate in the complex plane with the carrier-induced frequency-shift. The second term, $i 2 \pi \Delta \nu_{\text {inj }}$ then transforms the electric field into the frame of the injected signal. When $\widetilde{E}$ reaches a steady-state in the case with optical injection, the laser field is thus phase-locked to the injected signal and its frequency matches that of the master laser. Furthermore, as we now inject a real-valued signal into the laser cavity, the complex phase of $\widetilde{E}$ immediately gives the phase difference between the master and slave laser.

The remaining laser equations are unaffected by this change of rotating frame, as only the absolute square $|E|^{2} \equiv|\widetilde{E}|^{2}$ enters the charge-carrier equations.

\subsubsection{Injection Locking of Quantum-Dot Lasers}

We now implement Eq. (3.71) and simulate the quantum-dot laser under optical injection. The additional term in Eq. (3.71) describing the optical injection adds two new parameters to the system, the injection strength $K$ and the detuning $\Delta \nu_{\text {inj }}$. In order to characterize the dynamics of the laser with respect to these parameters, we create numerical bifurcation diagrams in the parameter plane spanned by $\left(K, \Delta \nu_{\text {inj }}\right)$. In order to characterize the laser dynamics, we distinguish between steady-state and oscillating solutions. Additionally, we determine the periodicity of the oscillations by evaluating the number of local extrema in the electric field amplitude during one oscillation. This allows us to perform a characterization of the injection-induced dynamics in dependence of the injection parameters. The resulting bifurcation diagram for the considered quantum-dot laser device is shown in Fig. 3.19.

Immediately visible is the triangular-shaped region of phase-locking, converging towards $K=0, \Delta \nu_{\mathrm{inj}}=0$. Within this region, the slave laser is phase-locked to the injected master signal, i.e., the system reaches a steady-state in the rotating frame of the master laser. Here, the master laser "enslaves" the injected laser, i.e., the injected signal is strong enough to force the slave laser to assume the 


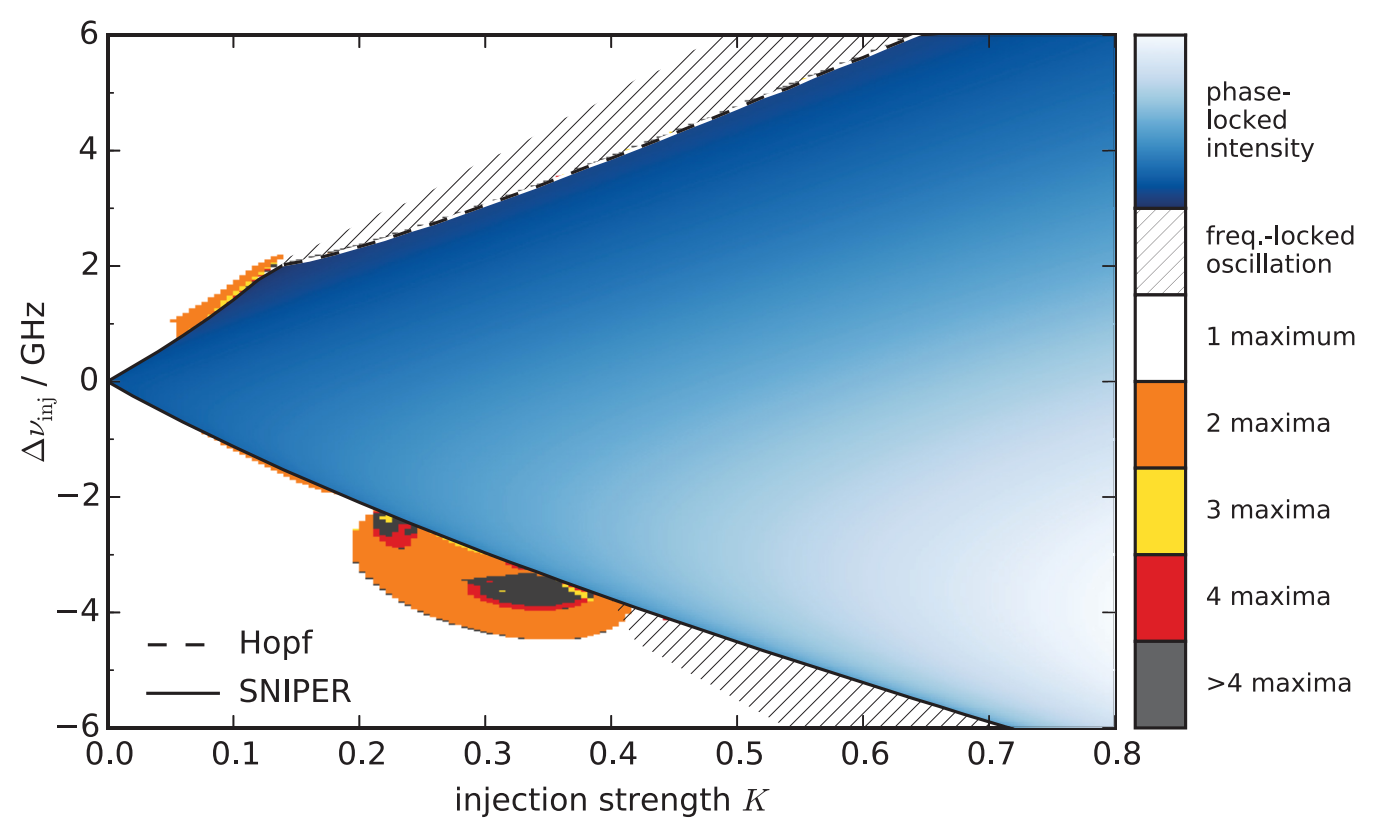

Figure 3.19.: Numerically simulated bifurcation diagram of the quantum-dot laser under optical injection. Shown are the dynamic regimes depending on the injection strength $K$ and the detuning $\Delta \nu_{\text {inj }}$. The solid and dashed lines denote SNIPER and Hopf bifurcations delimiting the triangular phase-locked region. The continuous color code inside this region shows the relative laser intensity from low (dark blue) to high intensity (light blue). Outside of the phase-locked region the laser exhibits oscillations. The white area denotes period-1 oscillations with one local maximum. The discrete color code denotes oscillations with two (orange), three (yellow) and four (red) maxima per oscillation period. Oscillations with higher number of maxima, an indication for chaotic and irregular oscillations, are shown by the dark gray color code. The hatched areas denote the regions where the laser is oscillating, but still frequency-locked to the injected signal. After [LIN13].

same wavelength. The frequency detuning interval for which this phase-locking is possible is in first order proportional to the injection strength $K$ [ARN65, ADL73]. This phenomenon shows the similarity between the optically injected quantum-dot laser and harmonically driven oscillators, from which such locking and synchronization effects are known [MUR05b].

When reaching the boundaries of this locking region for low $K \lesssim 0.14$, the stability of the phase-locked steady-state solution is lost in a saddle-node-infinite-period (SNIPER) bifurcation, denoted by the solid black lines in Fig. 3.19. Here, a stable fixed point collides with an unstable saddle, eliminating both fixed points in the process, and creating a periodic orbit. This process is sketched in Fig. 3.20. This mechanism of unlocking can be derived already in very simple models of driven 
(a)

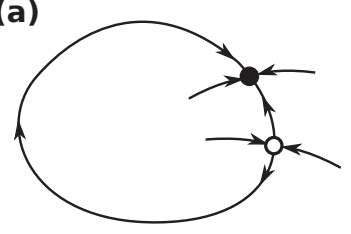

(b)

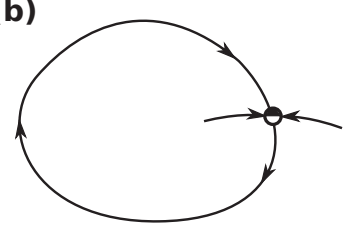

(c)

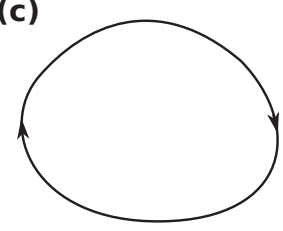

Figure 3.20.: Sketch of a saddle-node-infinite-period (SNIPER) bifurcation. (a) A stable fixed point (full circle) and an unstable saddle (empty circle) exist with a heteroclinic connection: the unstable manifold of the saddle is a stable manifold of the stable node. (b) At the bifurcation point the nodes collide, forming a homoclinic connection. (c) After the bifurcation, the fixed points have been annihilated and a periodic orbit was born from the former homoclinic connection.

phase oscillators [ADL73]. While the numeric evaluation of the injected quantumdot laser dynamics does not deliver an immediate conclusion about the involved bifurcation, the similarity of these systems already give an indication of the underlying dynamics. We will show later on by using numeric path-continuation techniques that here indeed a saddle-node bifurcation is responsible for the loss of phase-locking. Close to this SNIPER bifurcation, the injected laser has been shown to be excitable [GOU07, KEL09, KEL11]. This can be understood by the close distance of the stable node and the saddle in phase-space. When the laser operates on the stable fixed point, a small perturbation - either by an external trigger signal or noise [ZIE13] - will be able to drive the system across the unstable node and induce a large excursion along the heteroclinic connection. This excitability makes optically injected lasers an interesting system for nonlinear dynamics studies, e.g., as "optical neurons" [Mos00].

For higher injection strengths, the phase-locking region is delimited by a Hopf bifurcation of the stable fixed point on the positive detuning side. At the collision point of the SNIPER and Hopf bifurcation lines exists a codimension-2 saddle-nodeHopf point [KRA97b, ZIM97a, NIZ01], where the Hopf bifurcation changes its criticality from subcritical to supercritical for higher $K$, as we will see later on. From there on, at the locking boundary the stability of the stable fixed point is lost in the supercritical Hopf bifurcation, in which a stable limit cycle is born out of the fixed point.

The periodic orbit born in the Hopf bifurcation is qualitatively different from those born in the SNIPER bifurcations. This becomes obvious from its phase dynamics. We can evaluate the optical frequency of the slave laser by looking at the time evolution of its electric field phase $\widetilde{\phi}$ :

$$
\left\langle\Delta \nu_{\text {las }}\right\rangle:=\left\langle-\frac{\mathrm{d}}{\mathrm{d} t} \widetilde{\phi}\right\rangle=\left\langle-\operatorname{Im}\left(\frac{\frac{\mathrm{d}}{\mathrm{d} t} \widetilde{E}}{\widetilde{E}}\right)\right\rangle,
$$


where $\langle\cdot\rangle$ means the time average. The quantity $\left\langle\Delta \nu_{\text {las }}\right\rangle$ then describes the average frequency detuning of the injected laser from the master signal. In the phase-locked region $\left\langle\Delta \nu_{\text {las }}\right\rangle$ is zero, since the laser is in a steady-state, and $\frac{\mathrm{d}}{\mathrm{d} t} \widetilde{\phi}=0$. However, there can also be oscillating solutions that yield $\left\langle\Delta \nu_{\text {las }}\right\rangle=0$, even for $\frac{\mathrm{d}}{\mathrm{d} t} \widetilde{\phi} \neq 0$. As long as the optical field phase is bounded, the time-averaged phase-change is zero. Then, also $\left\langle\Delta \nu_{\text {las }}\right\rangle=0$, meaning that the optical frequency of the slave laser is still locked to the injected signal, while the intensity performs periodic oscillations. The hatched lines in Fig. 3.19 denotes these regions of frequency-locked or phasebounded oscillations [THE11, KEL12].

The transition from a phase-bounded to phase-unbounded oscillations is illustrated in Fig. 3.21. Here, we plot the time-series of the laser output power for $K=5$ and $\Delta \nu_{\text {inj }}=-5 \mathrm{GHz}$, where the oscillations are phase-bounded, as well as for $\Delta \nu_{\text {inj }}=-6 \mathrm{GHz}$, where they are unbounded. The time-series Fig. $3.21 \mathrm{a}, \mathrm{d}$ look very similar between the two cases. The trajectories in the complex electric field plane, Fig. 3.21 b,e, reveal the qualitative difference. The oscillation for $\Delta \nu_{\text {inj }}=-5 \mathrm{GHz}$ is still phase-bounded, i.e., the periodic orbit does not include the origin of the complex plane. The phase therefore oscillates between two extreme, but no full revolutions occur. For $\Delta \nu_{\text {inj }}=-6 \mathrm{GHz}$, on the other hand, the origin lies within the periodic orbit and thus every oscillation period correspond to a $2 \pi$ phase-shift. Here, the oscillation is thus unbounded and the mean output frequency of the laser becomes unlocked from the master signal.

The phase-unbounding transition is also visible in the optical spectrum of the laser output, shown in Fig. 3.21 c,f. Here, we evaluate the optical spectrum of the laser output by a Fourier-transform of the electric field,

$$
P(f)=\left|\int \mathrm{d} t \widetilde{E}(t) e^{-2 \pi f t}\right|^{2} .
$$

Since we chose the frequency of the master laser as the reference frame for $\widetilde{E}$, in the spectrum $f=0$ then refers to the master laser frequency.

Within the phase-bounded region the spectrum is dominated by the frequency of the master laser, with a smaller beat-signal contribution centered at the freerunning laser frequency. The phase-unbounding then describes the point where the former beat note becomes the dominant peak, i.e., here the laser returns to its free-running frequency (with a slight offset due to frequency-pulling). The transition point has recently been found to be of significant technological importance. Near this point noise effects can easily introduce strong phase-slips, depending on whether the noise drives the periodic orbit across the complex origin. Thus, the phase-noise exhibits a pronounced peak around this transition [ROM14], which worsens the laser linewidth and coherence. 

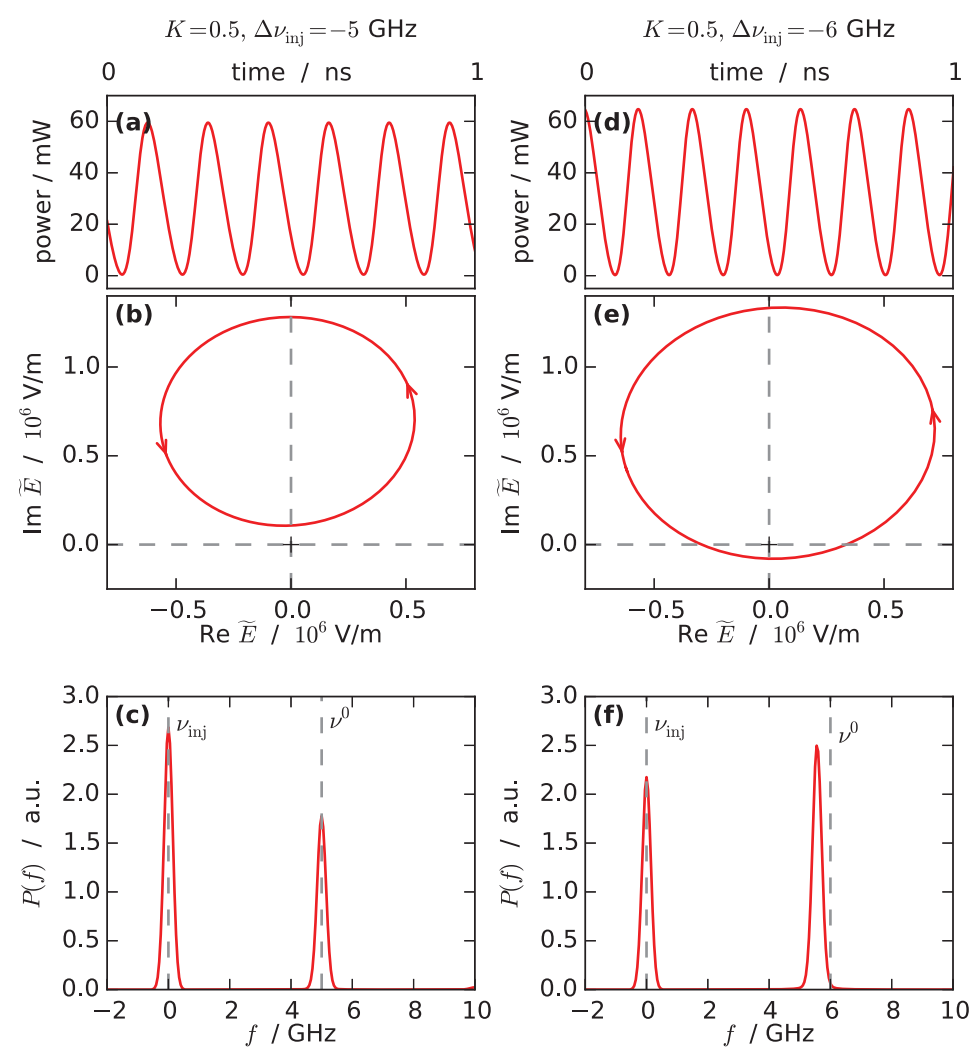

Figure 3.21.: Phase-unbounding of the optically injected quantum-dot laser after a Hopf bifurcation. (a) Output power time-series of the optically injected quantum-dot laser for $K=0.5, \Delta \nu_{\text {inj }}=-5 \mathrm{GHz}$, (b) corresponding periodic orbit in the complex electric field plane. The complex origin is marked by the dashed lines. (c) Optical power spectrum $P(f)$ of the laser output. The frequency $f=0$ corresponds to the master laser frequency $\nu_{\mathrm{inj}}$. The free-running laser frequency is marked by $\nu^{0}$. The spectrum was calculated with an artificial $350 \mathrm{MHz}$ detector resolution. (d),(e),(f) show the corresponding plots for $\Delta \nu_{\mathrm{inj}}=6 \mathrm{GHz}$.

Apart from the near-harmonic oscillations dominated by the beat-note between the injection signal and the slave laser, more complex oscillating solutions are observed in the bifurcation diagram Fig. 3.19. Most prominent is the elliptic region of higher periodicity located around $K=0.3, \Delta \nu_{\text {inj }}=-3 \mathrm{GHz}$. Here, bifurcations of the periodic orbits lead to highly complex dynamics, due to the near-resonant excitation of relaxation-oscillations in the quantum-dot laser. Lying within bubbles of highly-periodic oscillations there exist two regions of chaotic dynamics. The generation mechanisms of such chaotic regions can be manifold [WIE05]. For $K=0.3$ we find a period-doubling route to chaos when increasing the detuning $\Delta \nu_{\text {inj }}$ from 


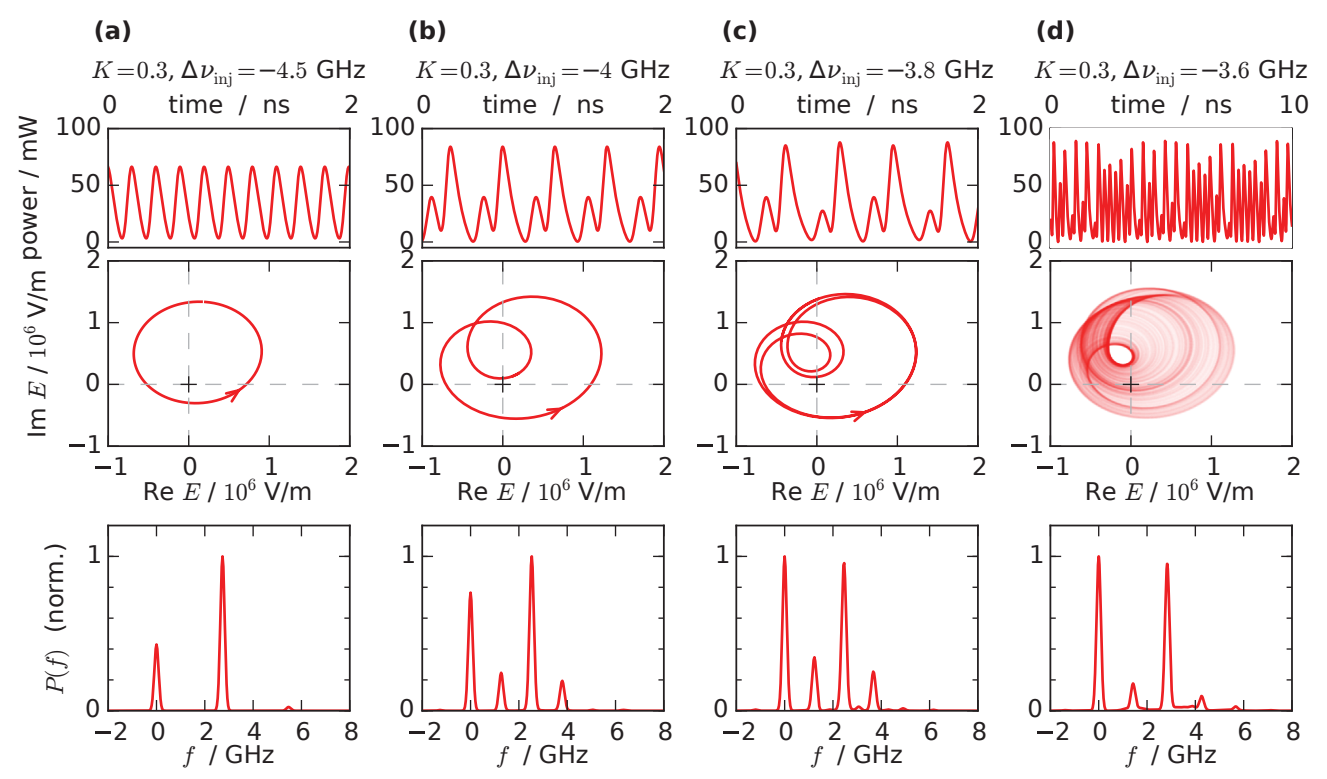

Figure 3.22.: Period-doubling bifurcations to chaos. Shown are the power time-series (top), trajectories in the complex electric field plane (middle), and the optical spectra relative to the master laser frequency. Shown are the dynamics for $\Delta \nu_{\text {inj }} \in$ $\{-4.5,-4,-3.8,-3.6\} \mathrm{GHz}((\mathbf{a})$ to $(\mathbf{d}))$ and $K=0.3$, cf. Fig. 3.21 .

negative values outside of the period-2-oscillation region. This is illustrated in Fig. 3.22.

Starting at $\Delta \nu_{\text {inj }}=-4.5 \mathrm{GHz}$, we encounter period-1 oscillations with the distinct beat note in the optical spectrum. Once we cross the period-doubling bifurcation, at $\Delta \nu_{\mathrm{inj}}=-4 \mathrm{GHz}$, the periodic orbit breaks into a dual loop. This is accompanied by the appearance of subharmonic peaks in the optical spectrum at half the distance between the two main peaks, due to the doubling of oscillation period. A period-4 oscillation is shown for $\Delta \nu_{\mathrm{inj}}=-3.8 \mathrm{GHz}$, until finally the chaotic attractor is born from the period-doubling cascade, following an infinite number of further period-doubling bifurcations.

Fig. 3.19 also shows a region of higher periodicity around $K=0.1$ at either side of the locking region. A further look into the periodic orbits involved in these regions, however, reveals that here no bifurcation occurs. Instead, the appearance of relaxation oscillations induces additional extrema in the output power. The recorded higher number of maxima is therefore a consequence of a deformation of the periodic orbits and no indication of period-doubling bifurcations. The chosen evaluation routine thus can not discriminate complex trajectory shapes from higher periodicity. The corresponding periodic orbits are shown in Fig. 3.23. 

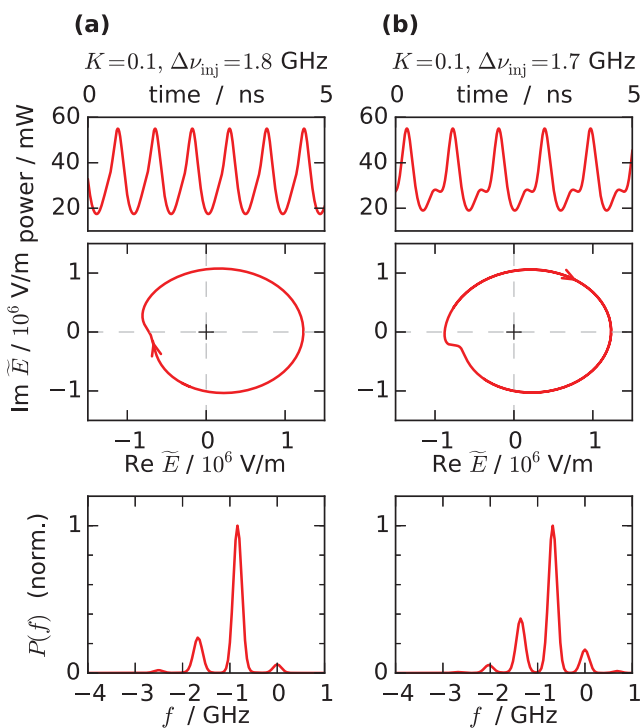

(b)

$K=0.1, \Delta \nu_{\text {inj }}=1.7 \mathrm{GHz}$

(c)

$K=0.1, \Delta \nu_{\text {inj }}=1.5 \mathrm{GHz}$
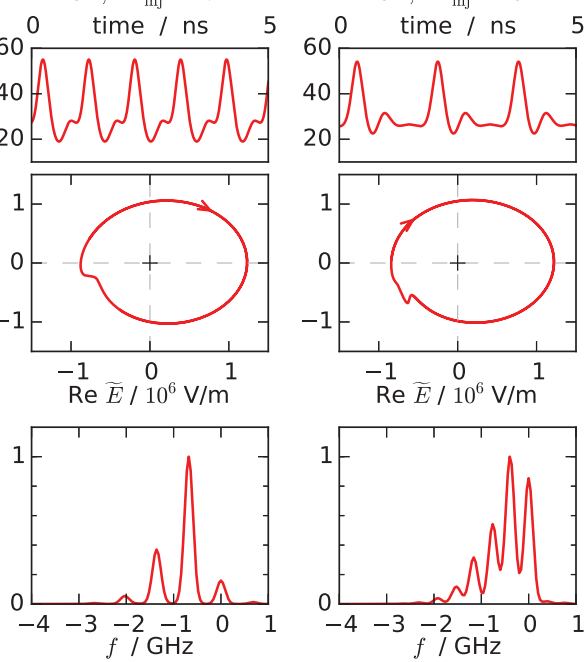

Figure 3.23.: Additional maxima due to deformation of the periodic orbit. Shown are the power time-series (top), trajectories in the complex electric field plane (middle), and the optical spectra relative to the master laser frequency. Shown are the dynamics for $\Delta \nu_{\text {inj }} \in\{1.8,1.7,1.5\} \mathrm{GHz}((\mathbf{a})$ to (c)) and $K=0.1$, cf. Fig. 3.21.

\subsubsection{Dependence on the Quantum-Dot Structure and Pump-Current}

In the previous section we have presented the rich nonlinear dynamics of the optically injected quantum-dot laser. We will now investigate the dependence of the bifurcation structure on key parameters of the quantum-dot laser. The easiest parameter to control is the pump current driving the quantum-dot laser. As we have shown before, the pump current influences the charge-carrier dynamics in the system by a change of the reservoir charge-carrier densities and the subsequent change of the individual scattering-rates.

When comparing different quantum-dot structures, a change of material composition or crystal growth parameters can change the underlying energy structure [BIM08a]. In addition to the quantum-dot device we have considered so far, we will investigate a different structure, which we will refer to as "deep-dot". The difference to the previously discussed structure (the "shallow-dot" structure, see Fig. 3.2) lies in the different energy levels, as shown in Fig. 3.24, with a deeper confinement of the quantum-dot states.

We now proceed by evaluating the bifurcation diagrams of both quantum-dot laser structures at different pump-currents. For the shallow-dot quantum-dot laser the resulting bifurcation diagrams are shown in Fig. 3.25. Here, we compare the 
Figure 3.24: Energy scheme of the localized quantumdot states for the deep-dot structure. The energy spacings are given in $\mathrm{meV}$. The reservoir band edges are shown by the light-blue shaded areas.

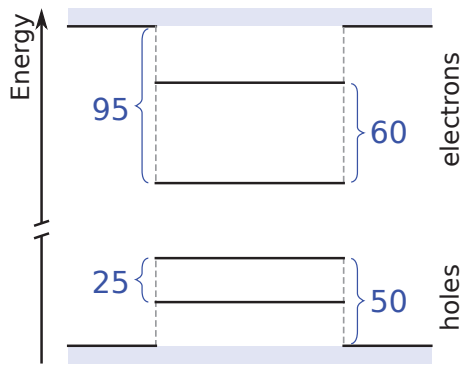

dynamics of the optically injected laser for $J=2 J_{\mathrm{th}}$, which we had already shown in Fig. 3.19, with the resulting dynamics at $J=5 J_{\text {th }}$.

Comparing the two bifurcation diagrams, a shift of the saddle-node-Hopf point for positive detuning towards higher injection strengths can be observed, along with a similar shift of the period-doubling region on the negative-detuning side outside of the locking region. We can understand this behavior from the dependence of the quantum-dot laser relaxation oscillation parameters on the pump current, which we have investigated in Sec. 3.2. With increasing pump-current, the relaxation oscillation damping and frequency both increase. It has been previously shown that the injection strength at which the saddle-node-Hopf point is located increases nearly linearly with the relaxation oscillation damping rate [OTT14]. This is in line with the interpretation of the excitation and undamping of relaxation oscillations by the injected signal, as the initial relaxation-oscillation damping must be overcome by the injected signal.

The period-doubling region outside of the locking region for negative detuning is shifted towards higher $K$ and enlarged nearly proportionally. The internal structure, however, remains the same, with two pronounced chaotic regions located inside the ellipse, suggesting a high robustness of the global bifurcation structure towards changes in the pump current [PAU12].

We now simulate the bifurcation diagrams for the deep-dot structure, again at twice and five times the threshold current. This is shown in Fig. 3.26. The bifurcation diagrams reveal a generally similar shape, with a triangular phase-locking region and regions of more complex oscillatory dynamics on the outside. Here, however, both the onset of the Hopf bifurcation on the positive detuning side of the locking region as well as the period-doubling regions are shifted towards lower absolute $K$, due to the lower relaxation oscillation damping rate of the quantum-dot laser with deeply confined energy levels.

The bifurcation structure inside of the period-doubling ellipse on the lower half of the bifurcation diagram reveals a much more involved arrangement of bifurcations. An extensive discussion of the dynamical details of this structure can be very complicated [WIE05] and goes beyond the scope of this work. Nevertheless, we 
(a)

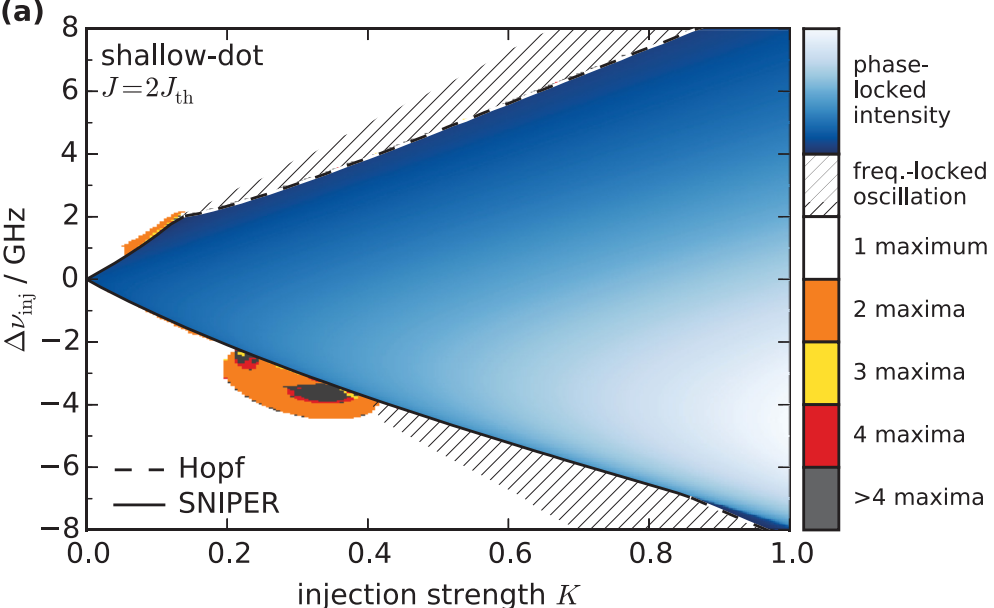

(b)

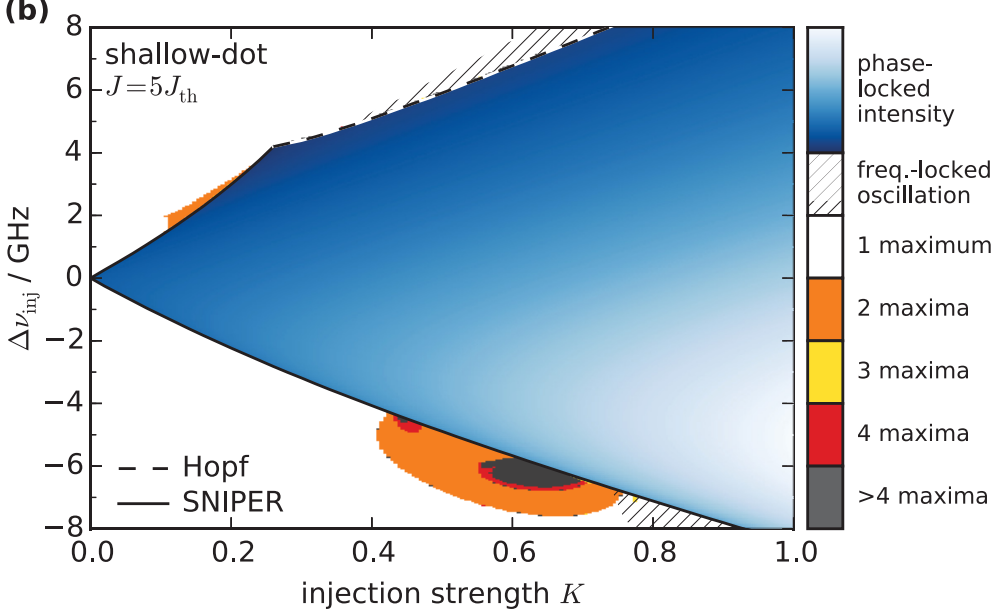

Figure 3.25.: Numerically simulated bifurcation diagram of the shallow-dot quantum-dot laser under optical injection, cf. Fig. 3.19. Shown are the bifurcation diagrams for (a) $J=2 J_{\mathrm{th}}$, and (b) $J=5 J_{\mathrm{th}}$. After [LIN13].

can again see that an increase in the pump current leads to the same shift of the bifurcations towards higher $K$ that we have already seen for the shallow-dot laser, while keeping the overall structure the same. It is interesting to note that for the deeply confined quantum-dots there exists an additional period-doubling region for positive detuning, close to the saddle-node-Hopf point. This region, however, overlaps with the region of induced relaxation peaks that we have discussed earlier (see Fig. 3.23), making a separation of these two effects difficult.

We have now gotten a first impression of the quantum-dot laser dynamics under optical injection. In the following, we will discuss the impact of the amplitudephase coupling in the quantum-dot laser on its dynamics, by comparing our full model to the dynamics that conventional models predict. 

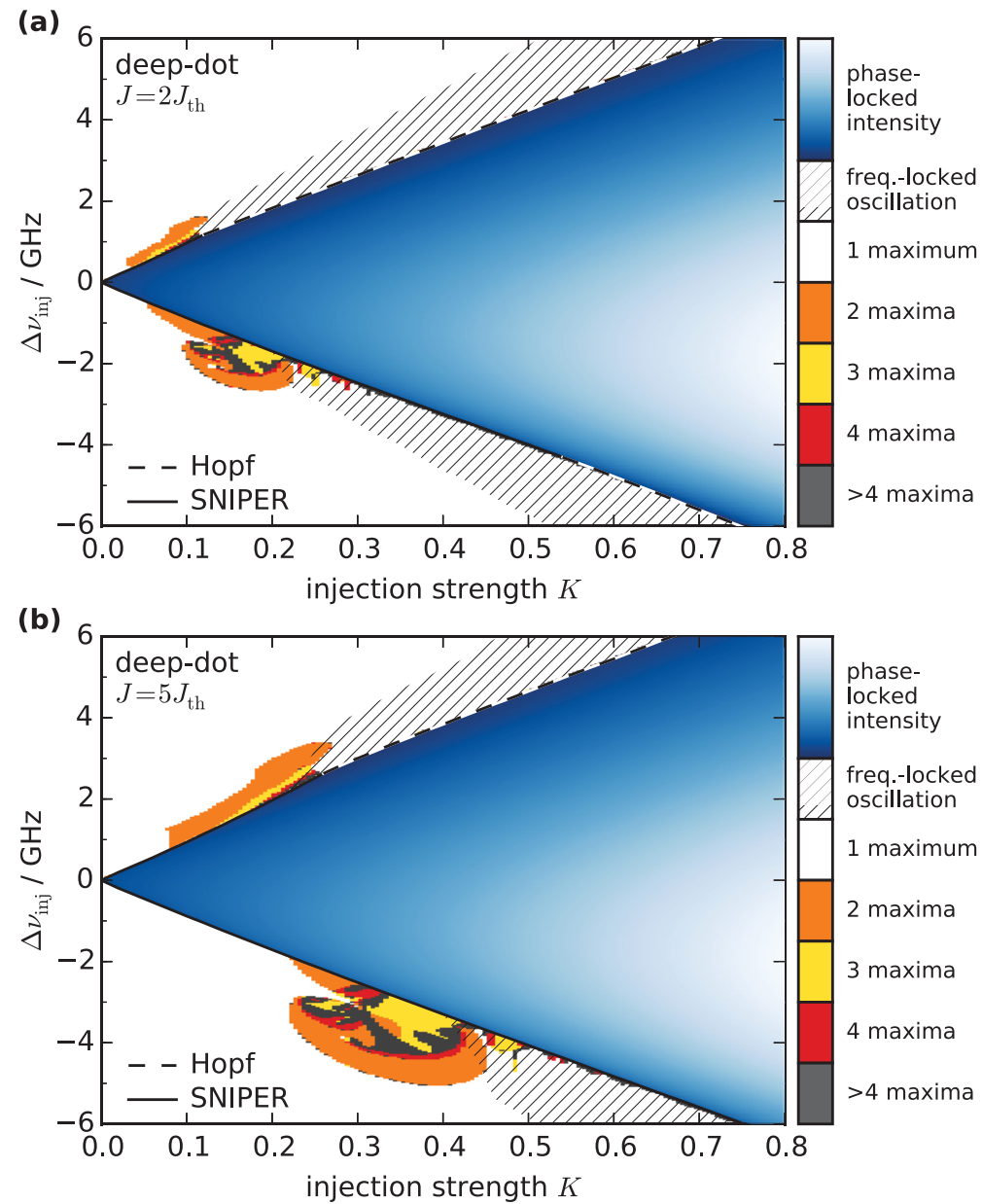

Figure 3.26.: Numerically simulated bifurcation diagram of the deep-dot quantum-dot laser under optical injection, cf. Fig. 3.19. Shown are the bifurcation diagrams for (a) $J=2 J_{\mathrm{th}}$, and (b) $J=5 J_{\mathrm{th}}$. After [LIN13].

\subsubsection{Evaluation of the $\alpha$-Factor from Optical Injection}

From the discussion of the injection-locking dynamics of the quantum-dot laser in the previous section we can conclude that quantum-dot lasers possess a similar general response to the injection of a master-laser signal, when compared to conventional laser devices. This includes a region of phase-locking for not too large detuning and areas of highly complex dynamics outside of this region.

In the following, we will thus try to describe the quantum-dot laser dynamics in the best way possible using a conventional modeling approach, i.e., using an $\alpha$-factor to describe the amplitude-phase coupling. As we have shown earlier, the 
$\alpha$-factor implements a linear dependence of the carrier-induced index change on the optical gain:

$$
\operatorname{Im} g(t) \equiv-\alpha \operatorname{Re} g(t)
$$

We will therefore rewrite Eq. (3.71) as

$$
\frac{\mathrm{d}}{\mathrm{d} t} \widetilde{E}(t)=(\operatorname{Re} g(t)-\kappa)(1-i \alpha) \widetilde{E}+i 2 \pi \Delta \nu_{\mathrm{inj}} \widetilde{E}+K \kappa E^{0},
$$

where we have exploited the fact that the frequency shift $\omega^{0}$ of the free-running laser is given by $\omega^{0}=\alpha \operatorname{Re} g^{0}=\alpha \kappa$ due to the gain-clamping in the steady-state. While neglecting the complex charge-carrier dynamics which determines the frequencyshift in the full model, the $\alpha$-factor approach greatly simplifies the dynamics of the electric field. Using only the field dynamics Eq. (3.75), we can derive an analytical expression for the saddle-node bifurcation delimiting the phase-locking region in dependence of $K$ and $\Delta \nu_{\text {inj. }}$.

Assuming that the laser is in a phase-locked steady-state, the time-derivative of $\widetilde{E}$ must vanish:

$$
0=(\operatorname{Re} g(t)-\kappa)(1-i \alpha) \widetilde{E}+i 2 \pi \Delta \nu_{\text {inj }} \widetilde{E}+K \kappa E^{0} .
$$

By splitting the above equation into its amplitude $\widetilde{A}$ and complex phase $\widetilde{\phi}$, we arrive at

$$
\begin{aligned}
0 & =\frac{\mathrm{d}}{\mathrm{d} t} \widetilde{\phi}(t)=-\alpha(\operatorname{Re} g(t)-\kappa)+2 \pi \Delta \nu_{\mathrm{inj}}-\sin \widetilde{\phi} \frac{K \kappa E^{0}}{\widetilde{A}} \\
0 & =\frac{\mathrm{d}}{\mathrm{d} t} \widetilde{A}(t)=(\operatorname{Re} g(t)-\kappa) \widetilde{A}+\cos \widetilde{\phi} K \kappa E^{0} .
\end{aligned}
$$

Inserting Eq. (3.77b) into Eq. (3.77a), we can rewrite it as

$$
2 \pi \Delta \nu_{\mathrm{inj}}=\frac{K \kappa E^{0}}{\widetilde{A}}[-\alpha \cos \widetilde{\phi}+\sin \widetilde{\phi}]
$$

Utilizing the trigonometric identity $\sin (\phi)-\alpha \cos (\phi)=\sqrt{1+\alpha^{2}} \sin (\phi-\arctan \alpha)$, we can write

$$
2 \pi \Delta \nu_{\mathrm{inj}} \frac{\widetilde{A}}{K \kappa E^{0} \sqrt{1+\alpha^{2}}}=\sin (\widetilde{\phi}-\arctan \alpha)
$$




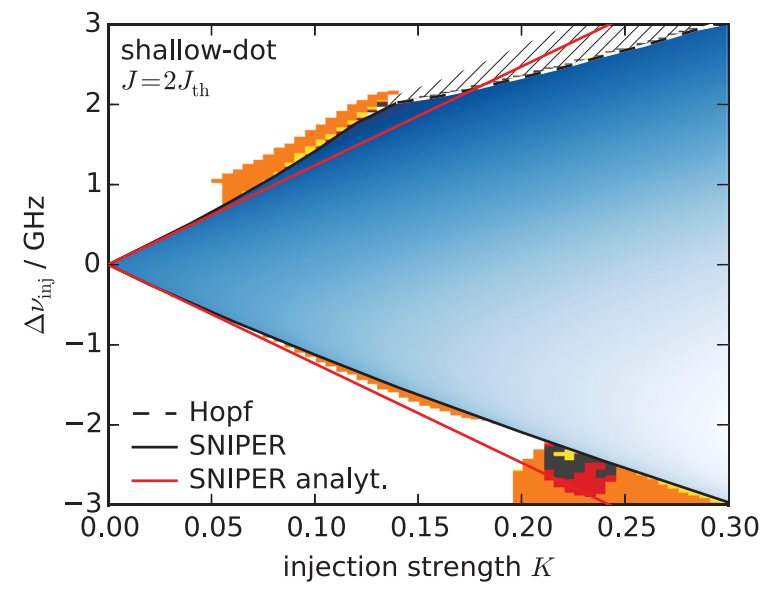

Figure 3.27.: Bifurcation diagram of the optically injected shallow-dot laser with the analytical expression Eq. (3.80) for the SNIPER bifurcation line (red), using $\alpha \equiv \alpha_{\mathrm{inj}}=$ 1.19. Cf. Fig. 3.19.

which admits only solutions if the left-hand side stays within $[-1,1]$. From this, we acquire the locking boundaries as

$$
\Delta \nu_{\text {inj }}^{\text {lock }}= \pm \frac{K \kappa}{2 \pi} \frac{E^{0}}{\widetilde{A}} \sqrt{1+\alpha^{2}} \approx \pm \frac{K \kappa}{2 \pi} \sqrt{1+\alpha^{2}}
$$

where the last term approximates the locking boundaries for $\widetilde{A} \approx E^{0}$, i.e., for low injection strengths. Eq. (3.80) describes the linear increase of the locking range with the injection strength $K$ that we have already observed in the numerical evaluation of the bifurcation structure. Under the assumption of constant electricfield amplitude and $\alpha=0$, the above equation would reproduce the phase-locking behavior known from Adler's equation [ADL73], showing the connection between the optically injected quantum-laser and simple driven phase oscillators.

Eq. (3.80) gives an expression for the locking boundaries in dependence on the parameter $\alpha$. We can thus fit our numerical results for the SNIPER bifurcation lines using the above expression to extract a value for $\alpha$. For the shallow-dot laser device at twice the threshold current we obtain $\alpha \equiv \alpha_{\text {inj }}=1.19$. We use the symbol $\alpha_{\text {inj }}$ to label the $\alpha$-factor extracted from the optical injection simulations.

Fig. 3.27 shows the comparison between the analytical approximation Eq. (3.80) and the numerically obtained bifurcation diagram. The analytical expression (red curve) shows perfect agreement with the numerical results for low injection strength. With increasing $K$, the numerically determined SNIPER bifurcation lines show a deviation towards higher $\Delta \nu_{\text {inj }}$. Here, the approximation $\widetilde{A} \approx E^{0}$ fails. In order to resolve this problem, one would need to derive an expression for $\widetilde{A}$ in dependence 
of the injection parameters, for which a consideration of the charge-carrier dynamics is required. Analytical expressions can be derived for simple carrier dynamics [ERN10b, OTT14], but are not feasible for our full modeling approach.

The approach used in determining $\alpha_{\text {inj }}$ by fitting the bifurcation lines apparently yields an accurate, but also rather complicated task. We can circumvent the fitting procedure by directly applying the definition of the $\alpha$-factor, Eq. (3.59), using the ratio of variation in the imaginary and real parts of the optical gain. As we have stressed before, this definition is in general not well-defined, as it depends on the individual charge-carrier occupation changes $\delta \rho$. In the present case of optical injection, we can however explicitly determine these changes from the numerics.

We thus evaluate the reaction of the quantum-dot laser towards a small change in the electric-field amplitude, realized by a change in the injection strength $K$ :

$$
\alpha_{\text {inj }}:=-\frac{\partial \operatorname{Im} g / \partial K}{\partial \operatorname{Re} g / \partial K}=-\frac{\left.\operatorname{Im} g\right|_{K=\Delta K}-\left.\operatorname{Im} g\right|_{K=0}}{\left.\operatorname{Re} g\right|_{K=\Delta K}-\left.\operatorname{Re} g\right|_{K=0}} .
$$

We evaluate this expression at $K=0$ and $\Delta \nu_{\text {inj }}=0$, i.e., the numerical derivatives $\partial / \partial K$ become the differences of the real and imaginary parts of the gain with respect to their values obtained for the free-running laser at $K=0$. Here it is important to note that we evaluate the adiabatic changes of the gain with the injection strength, i.e., we evaluate it after all transients have decayed and the laser has reached its new steady-state. When we perform this procedure using $\Delta K=10^{-4}$, we obtain $\alpha_{\mathrm{inj}}=1.19$, which is exactly the value received from the fit to the bifurcation diagram. We will thus evaluate Eq. (3.81) in order to extract an $\alpha$-factor that describes the locking behavior of the quantum-dot laser in the best way possible.

\subsubsection{Comparison with $\alpha$-Factor-Based Models}

\section{Shallow-Dots}

We now create bifurcation diagrams for the different cases we have studied before, but using an $\alpha$-factor approach to model the amplitude-phase coupling. We thus apply Eq. (3.81) and determine an effective $\alpha$-factor to describe the response of the quantum-dot laser to the optical injection. In the simulations we then implement Eq. (3.75) instead of calculating the index-changes from the off-resonant optical transitions.

For the shallow-dot case at twice the threshold current, we will at first compare the bifurcations delimiting the phase-locking region. The comparison between the modeling approaches is shown in Fig. 3.28. Here we can see a very good agreement between the two cases for the SNIPER bifurcation lines, which show only slight 


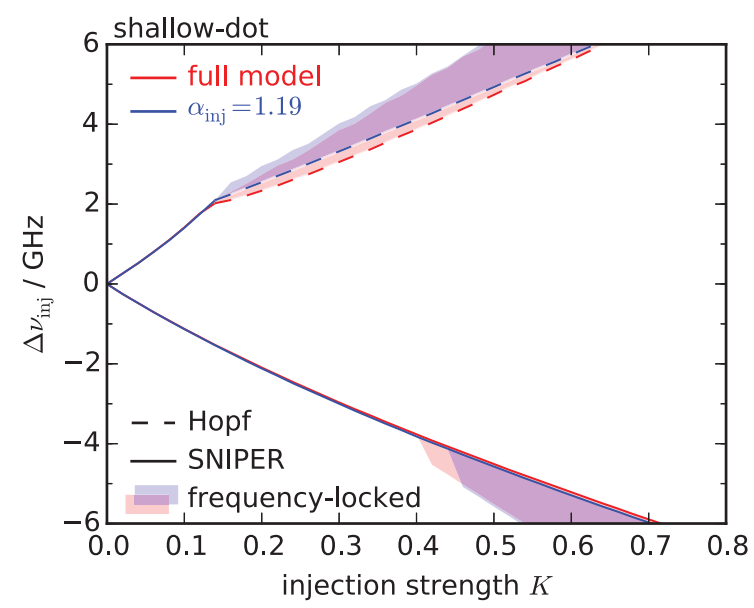

Figure 3.28.: Comparison of the injection-locking range for the shallow-dot laser under optical injection. Shown are the SNIPER (solid) and Hopf (dashed) bifurcation lines for the full model (red) and the $\alpha$-factor approach (blue). The shaded regions show frequency-locked oscillations. $J=2 J_{\text {th }}$.

differences at higher injection strength. The Hopf bifurcation lines on the other hand show a shift along the detuning axis, with the full model predicting a slightly smaller locking range at the positive detuning side. Along with the Hopf lines also the regions of frequency-locked oscillation are shifted. This good agreement shows us that our choice of $\alpha_{\text {inj }}$ does indeed reproduce the locking dynamics of the quantum-dot laser rather well.

We thus proceed to create the complete bifurcation diagram using the $\alpha$-factor model. The comparison between the two approaches is shown in Fig. 3.29. We can see that the $\alpha$-factor reproduces the same general shape of dynamics outside of the locking range, with a bubble of more complex dynamics outside of the lower locking boundary. However, the period-doubling ellipse is shifted towards higher $K$, and the bifurcation structure inside of this region can be seen to differ from the full model. Using an $\alpha$-factor, the chaotic region at smaller $K$ vanishes, and the other chaotic region differs in shape and size, with a rather pronounced region of period-3 oscillation appearing. Apart from this, the period-doubling region at positive detunings near the saddle-node-Hopf point, that was previously only seen in the deep-dot case, is now also visible for the shallow-dot laser. The bifurcation diagrams for $J=5 J_{\text {th }}$, Fig. 3.30, reproduce this behavior, showing similar difference between the two models. 
(a)
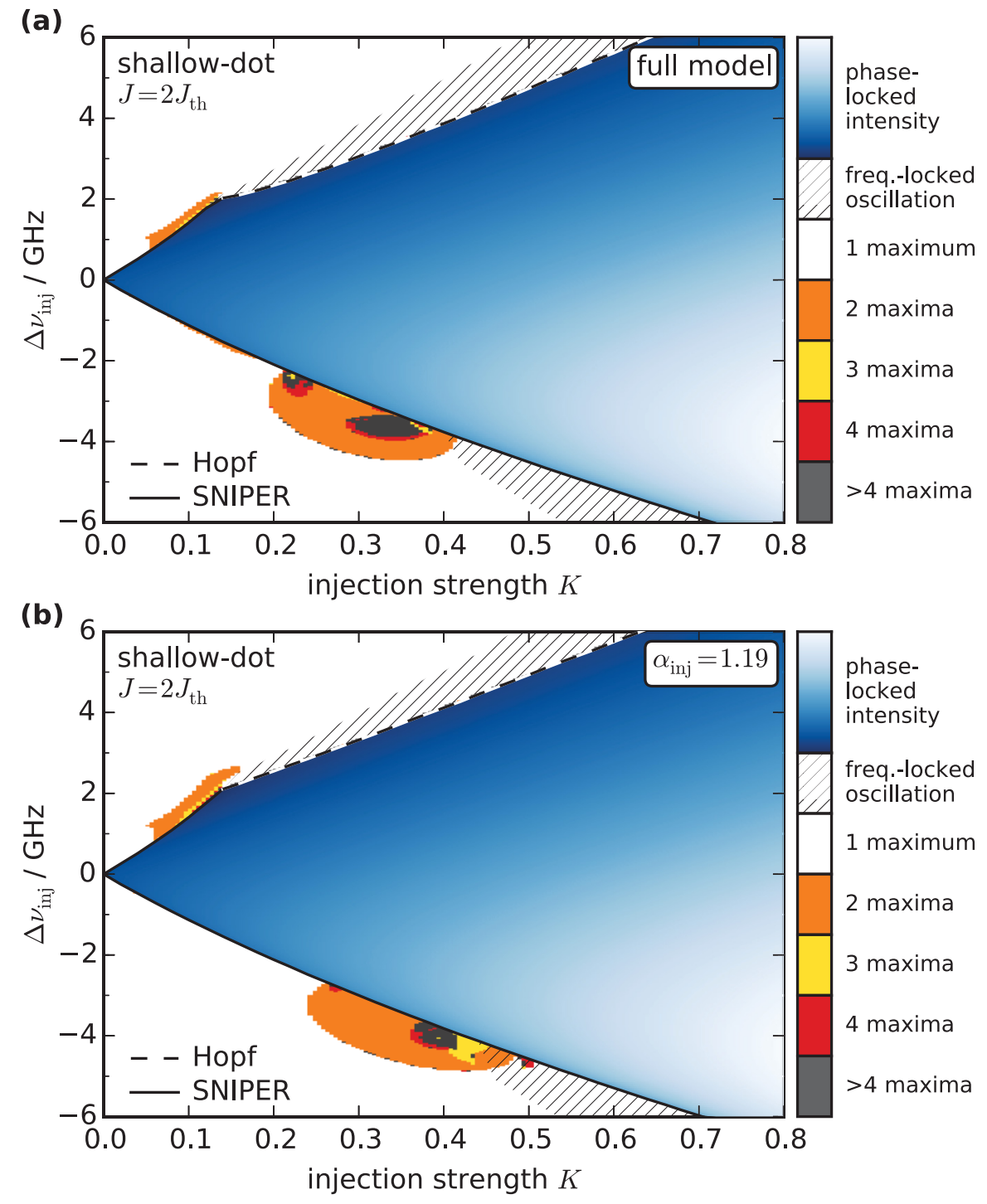

Figure 3.29.: Comparison of the bifurcation diagram for the shallow-dot laser under optical injection. (a) shows the bifurcation diagram obtained using the full model, Eq. (3.71), (b) $\alpha$-factor approach, Eq. (3.75). $J=2 J_{\text {th }}$, cf. Fig. 3.19. After [LIN13]. 

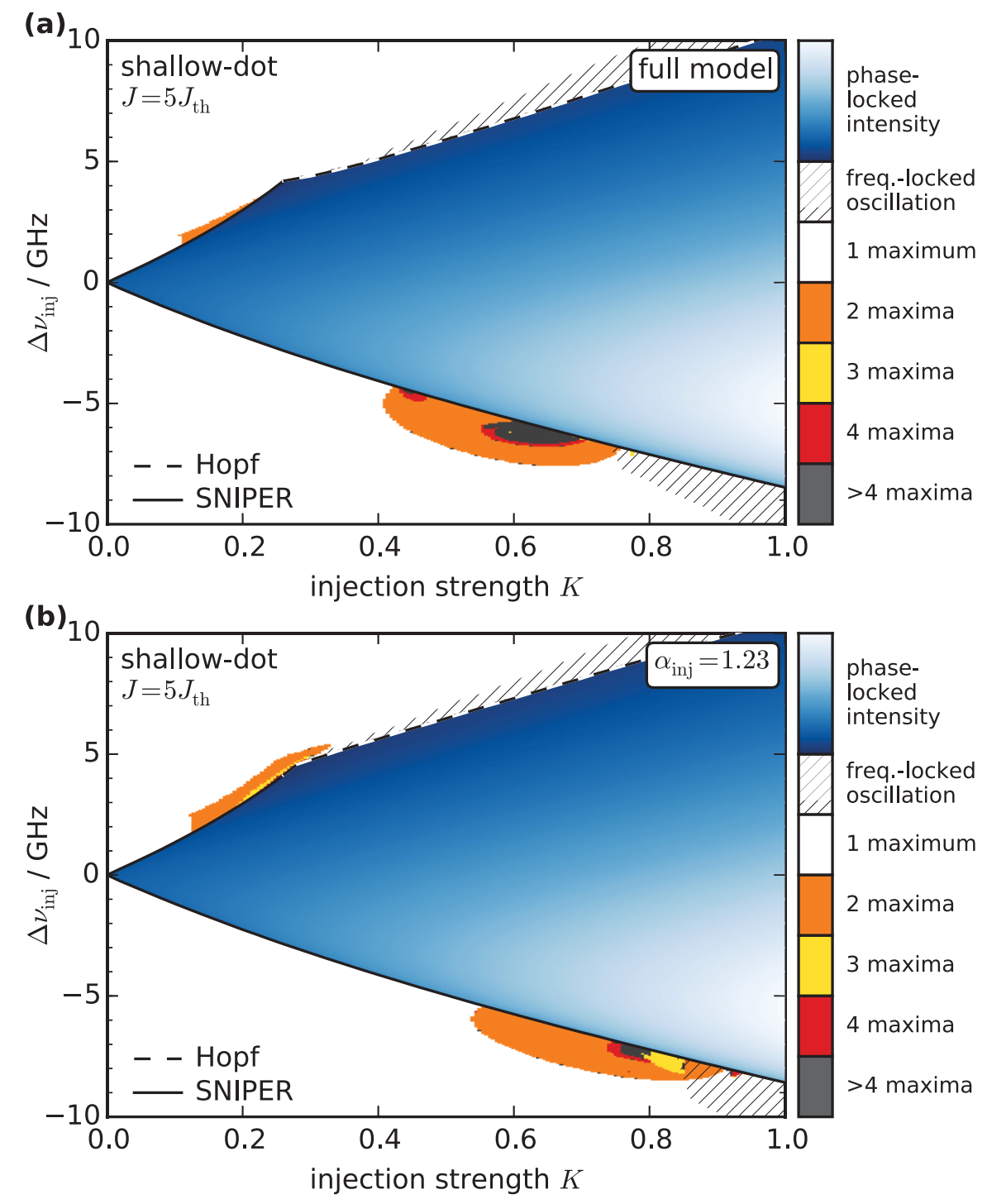

Figure 3.30.: Comparison of the bifurcation diagram for the shallow-dot laser under optical injection. (a) shows the bifurcation diagram obtained using the full model, Eq. (3.71), (b) $\alpha$-factor approach, Eq. (3.75). $J=5 J_{\text {th }}$, cf. Fig. 3.19. After [LIN13]. 

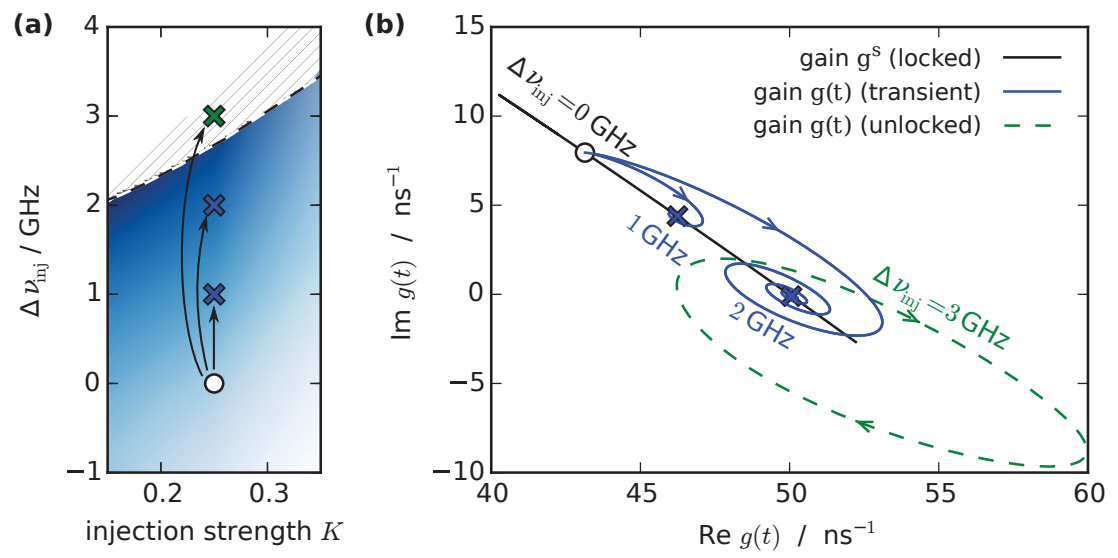

Figure 3.31.: Transients of the complex gain in the optically injected shallow-dot laser. The detuning $\Delta \nu_{\mathrm{inj}}$ is switched from $0 \mathrm{GHz}$ (filled circle in the section of the bifurcation diagram in (a)) to $1 \mathrm{GHz}, 2 \mathrm{GHz}$, or $3 \mathrm{GHz}$, respectively (crosses in (a)). (b) shows the corresponding transients $g(t)$ in the complex plane of the optical gain. The transients when switching to another phase-locked state are shown in blue, the green dashed line shows the periodic orbit outside of the locking range. The black line shows the steadystate gain values $g^{s}$ inside of the locking range for $K=0.25 . \quad J=2 J_{\mathrm{th}}$, cf. Fig. 3.19. After [LIN13].

The bifurcation diagrams show that the $\alpha$-factor approach reproduces the bifurcations of the steady-state solutions - the phase-locked states - rather well. The dynamic solutions, on the other hand, exhibit differences. We will try to understand this behavior by investigating the dynamics of the complex optical gain of the injected quantum-dot laser.

We thus look at the response of the quantum-dot laser to an instantaneous switching of the injection frequency. This is depicted in Fig. 3.31, where we have simulated the quantum-dot laser with injection at $K=0.25$ and $\Delta \nu_{\text {inj }}=0 \mathrm{GHz}$, such that the laser is in a phase-locked steady-state. From there, we have changed $\Delta \nu_{\text {inj }}$ to 1,2 , and $3 \mathrm{GHz}$, with $\Delta \nu_{\text {inj }}=3 \mathrm{GHz}$ lying outside the phase-locking region, the other two values within (see Fig. $3.31 \mathrm{a}$ ).

When switching to values of the detuning that lie within the locking range, the laser will reach a phase-locked steady-state after the transients have decayed (blue curves in Fig. $3.31 \mathrm{~b}$ ). The gain $g^{s}$ that is assumed in the steady-states for different $\Delta \nu_{\text {inj }}$ inside the phase-locked region are shown by the black line. As we can see, this curve is nearly linear, which would be in agreement with a constant $\alpha$-factor, giving a constant slope in the $(\operatorname{Re} g, \operatorname{Im} g)$-phase-space. The transients when switching to a different $\Delta \nu_{\text {inj }}$, however, reveal a deviation from this linear relationship. The periodic orbit outside of the locking-range for $\Delta \nu_{\mathrm{inj}}=3 \mathrm{GHz}$ (green dashed) as 
well as the transients can clearly not be described by a linear relationship between the real and imaginary parts of the optical gain.

We can understand this behavior by discussing the charge-carrier dynamics induced by the change of injection parameters. As long as we only look at the steadystate solutions, we can easily define a functional dependence of the charge-carrier distribution $\rho$, including all quantum-dot and reservoir states, on the injection parameters: $\rho \equiv \rho\left(K, \Delta \nu_{\text {inj }}\right)$. As the gain $g^{s}$ in the steady-state is a function of the carrier distribution, the derivative $\partial g^{s} / \partial \Delta \nu_{\text {inj }}$ exists, and we can define an $\alpha$-factor in terms of the derivatives of the real and imaginary parts of $g^{s}$ with respect to $\Delta \nu_{\text {inj }}$, similar to Eq. (3.81). As we have stressed before, this is no longer possible when looking at dynamic solutions, as the time-evolution of the gain can exhibit complicated dynamics itself, without the possibility to describe it by an $\alpha$-factor. This will become especially pronounced when discussing dynamically complex solutions where the electric field exhibits dynamics on the timescale of the charge-carrier lifetimes, where the carrier distribution is no longer able to adiabatically follow the electric field dynamics. Here, using an $\alpha$-factor would artificially constrain the dynamics of the gain in the phase-space to the black line in Fig. 3.31, which, as we have seen, will lead to inaccurate predictions of the dynamics.

The above discussion explains the differences between the full-modeling approach and the $\alpha$-factor model. The bifurcations of the steady-state solutions delimiting the locking region can be very well describe with an $\alpha$-factor, since the steady-state solutions adiabatically follow a nearly linear relationship in the complex gain. The dynamical solutions, on the other hand, cannot be described by an $\alpha$-factor, and their bifurcations thus differ between the two approaches.

It has become clear that the difference between quantum-dot laser dynamics and conventional lasers is strongly influenced by the amplitude-phase coupling. We will therefore take another look at the quantum-dot laser dynamics in terms of the amplitude-phase coupling by evaluating its gain and index dynamics. We thus extend the definition of $\alpha_{\text {inj }}$, Eq. (3.81), to the whole parameter space:

$$
\alpha_{\text {inj }}\left(K, \Delta \nu_{\text {inj }}\right):=-\frac{\partial \operatorname{Im} g^{s}\left(K, \Delta \nu_{\text {inj }}\right) / \partial K}{\partial \operatorname{Re} g^{s}\left(K, \Delta \nu_{\text {inj }}\right) / \partial K} .
$$

We must again note that this definition is only well-defined within the phase-locking region, where the laser reaches a steady-state. The definition for $\alpha_{\text {inj }}\left(K, \Delta \nu_{\text {inj }}\right)$ therefore is based on the derivatives of the steady-state value of the complex gain, $g^{s}\left(K, \Delta \nu_{\text {inj }}\right)$. When the laser is not phase-locked, a different definition has to be used. As we have seen in Fig. 3.31, the real and imaginary parts of $g(t)$ are desynchronized for oscillatory solutions, and the derivative $\partial \operatorname{Re} g(t) / \partial \operatorname{Im} g(t)$ cannot be 


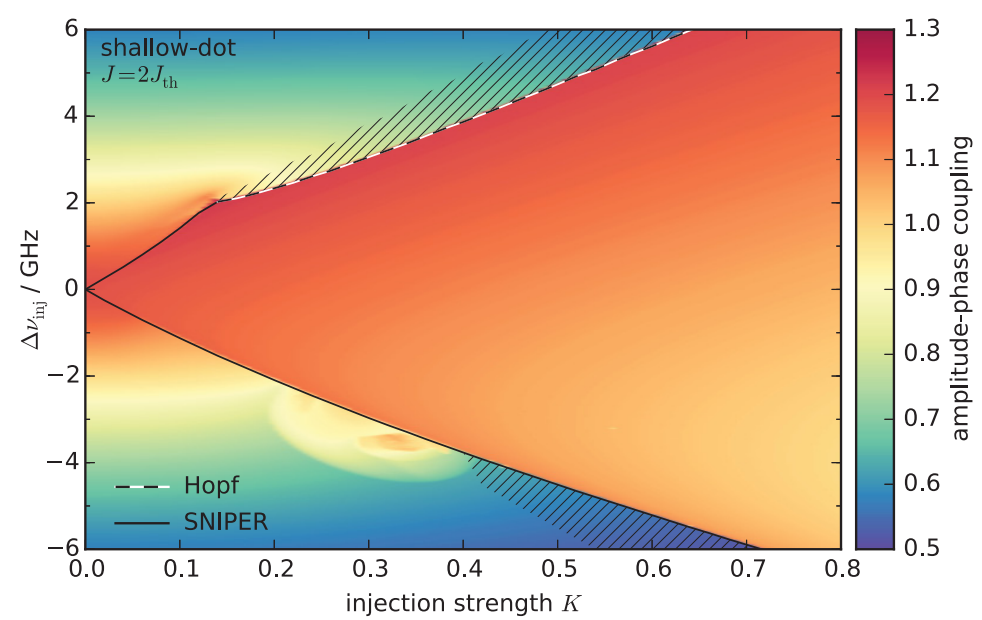

Figure 3.32.: Evaluation of the amplitude-phase coupling in the optically injected shallowdot laser. Within the phase-locked parameter region, we plot $\alpha_{\mathrm{inj}}$, Eq. (3.82), for dynamic solutions $\alpha_{\mathrm{inj}}^{\mathrm{dyn}}$, Eq. (3.84), is shown, with their values denoted by the color code. $J=2 J_{\text {th }}$, cf. Fig. 3.19 .

defined. Instead, we will evaluate the modulation amplitudes of these quantities, i.e., we define

$$
\begin{aligned}
\Delta \operatorname{Re} g\left(K, \Delta \nu_{\mathrm{inj}}\right) & :=[\max [\operatorname{Re} g(t)]-\min [\operatorname{Re} g(t)]]_{K, \Delta \nu_{\mathrm{inj}}} \\
\Delta \operatorname{Im} g\left(K, \Delta \nu_{\mathrm{inj}}\right) & :=[\max [\operatorname{Im} g(t)]-\min [\operatorname{Im} g(t)]]_{K, \Delta \nu_{\mathrm{inj}}}
\end{aligned}
$$

with their minimum and maximum values evaluated for the corresponding dynamic solution obtained at the parameter combination $\left(K, \Delta \nu_{\text {inj }}\right)$. We then evaluate their ratio,

$$
\alpha_{\mathrm{inj}}^{\mathrm{dyn}}\left(K, \Delta \nu_{\mathrm{inj}}\right):=\frac{\Delta \operatorname{Im} g\left(K, \Delta \nu_{\mathrm{inj}}\right)}{\Delta \operatorname{Re} g\left(K, \Delta \nu_{\mathrm{inj}}\right)} .
$$

in order to quantify the amplitude-phase coupling outside of the locking range.

We evaluate Eqs. (3.82), (3.84) for the shallow-dot laser under optical injection. The resulting values in dependence of the injection parameters are shown in Fig. 3.32. The value of $\alpha_{\text {inj }}$ within the locking region exhibits a decrease towards higher $K$, which coincides with an increase of the optical power. This can be understood in terms of the quantum-dot ground-state occupation. At higher intensity, the stimulated recombination rate becomes larger, forcing the quantum-dot occupation towards zero inversion. With reduced occupation, the gain saturation due to Pauli-blocking becomes less pronounced in the ground-state, such that a 
variation of the charge-carrier distribution leads to a higher relative change of the optical gain and thus to a smaller value of $\alpha_{\mathrm{inj}}$.

At the locking boundaries, the value of $\alpha_{\text {inj }}$ is nearly constant, and approximately equal to the value evaluated at $K=\Delta \nu_{\text {inj }}=0$. This explains the good agreement between the full gain dynamics and the description with an $\alpha$-factor, as here the full gain dynamics can be very well described with this constant $\alpha_{\text {inj }}$.

Outside of the locking region, however, the discrepancies become evident. While for small values of $K$ and $\Delta \nu_{\text {inj }}$ the values inside and outside of the locking region match rather well, there is a substantial decrease of $\alpha_{\mathrm{inj}}^{\text {dyn }}$ towards greater detuning frequencies $\left|\Delta \nu_{\text {inj }}\right|$. Furthermore, the laser dynamics itself can be seen to influence the value of $\alpha_{\mathrm{inj}}^{\mathrm{dyn}}$. The signatures of the different qualitative dynamics and their bifurcations within the elliptic region of higher periodicity at the lower injection-locking boundary are clearly visible also in the amplitude-phase coupling. This again shows that no single value of $\alpha$ can be defined for quantum-dot lasers that would be valid for all types of solutions, but a consistent and independent description of the optical gain and frequency-shift is required.

\section{Deep-Dot Laser}

We now also simulate the quantum-dot laser with deeply confined localized states and compare its dynamics with the results from the $\alpha$-factor description. The evaluation of $\alpha_{\text {inj }}$ yields a lower value of 0.55 at $J=5 J_{\text {th }}$ in this case. Compared to the shallow-dot laser, this reduced value can be explained by the larger energy spacing of the reservoir states from the quantum-dot ground-state, reducing their effect on the frequency shift at the ground-state energy.

Fig. 3.33 reveals the same general trend that we have already seen for the shallowdot laser. The simulations using an $\alpha$-factor can reproduce the bifurcations of the static phase-locked solution very well, while the bifurcations of the dynamic solutions are shifted in parameter space. Furthermore, the detailed bifurcation structure enclosed within the period-2 region at negative $\Delta \nu_{\text {inj }}$ exhibits quite substantial differences. It becomes again evident that when using an $\alpha$-factor for the simulation of quantum-dot laser dynamics, the amplitude-phase coupling cannot be accurately described. This holds especially for the complex bifurcation structure of the periodic solutions. As we will see later on in Sec. 3.9, this abrupt change of the amplitude-phase coupling between the locked and unlocked regions is due to a frequency-dependent modulation response of the carrier-induced frequencyshift. The amplitude-phase-coupling thus becomes subsequently smaller the faster the dynamics of the electric field becomes. This is also demonstrated in Fig. 3.34, which shows the amplitude-phase coupling in the injection parameter plane, as done before for the shallow-dot (cf. Fig. 3.32). Here we can clearly observe the 
(a)

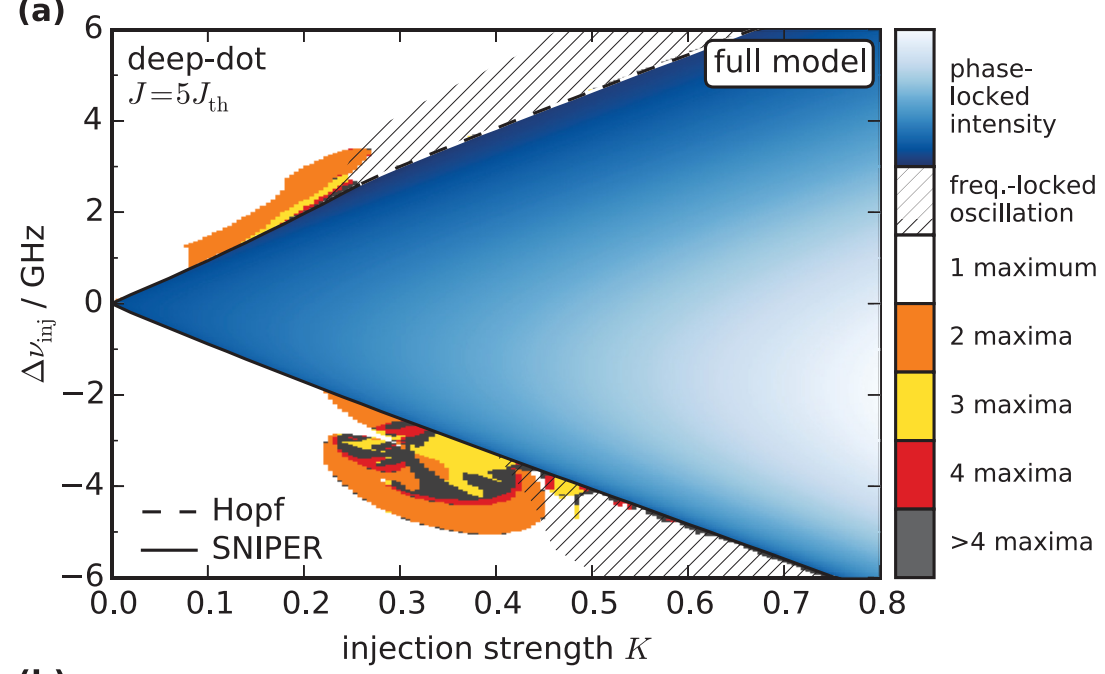

(b)

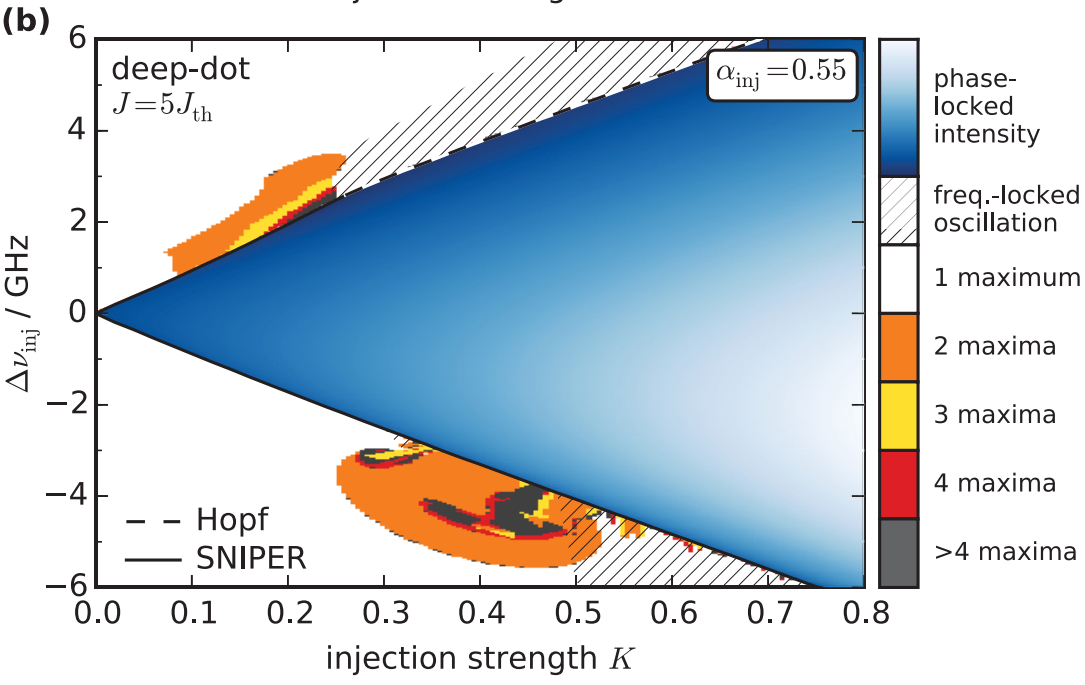

Figure 3.33.: Comparison of the bifurcation diagram for the deep-dot laser under optical injection. (a) shows the bifurcation diagram obtained using the full model, Eq. (3.71), (b) $\alpha$-factor approach, Eq. (3.75). $J=5 J_{\text {th }}$, cf. Fig. 3.19. After [LIN13].

reduction in amplitude-phase coupling as soon as we leave the phase-locked region, which then leads to the differences in the dynamics between the two modeling approaches.

Our numerical results by direct integration of our quantum-dot laser model shows that the unique dynamics of quantum-dot lasers lead to important modifications to the bifurcation structure. While the numerical bifurcation diagrams of the quantum-dot laser in the parameter plane gives an overview of the qualitative dynamics, a more general investigation of the dynamics would be favorable. A 


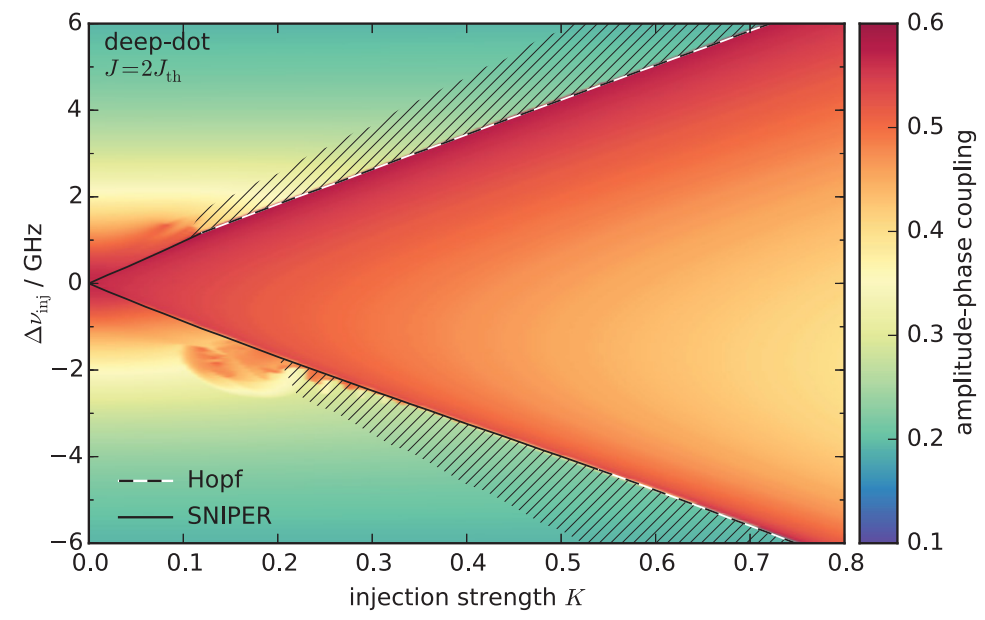

Figure 3.34.: Evaluation of the amplitude-phase coupling in the optically injected deepdot laser. $J=5 J_{\text {th }}$, cf. Fig. 3.32 .

detailed investigation of the bifurcations, however, requires a treatment that goes beyond the simple integration of the quantum-dot laser model. In the following section we will therefore present a simplification of our full model that is suitable for implementation in numerical path-continuation tools. 


\subsection{Optical Injection - Numerical Path Continuation}

While the numerical evaluation of bifurcation diagrams, as shown in the previous sections, can give an overview of the possible dynamics in the system, it has several drawbacks. The bifurcation diagrams were created by direct integration of the quantum-dot laser equations. Together with the high number of sampling points in the parameter space required for a sufficiently high resolution, this can lead to significant computational demands. Furthermore only stable solutions can be found by this approach. And in the case of multi-stability only one particular solution will be reached, with the remaining going potentially unnoticed.

A more elegant way of characterization of the occurring dynamics is offered by path-continuation programs. These makes it possible to trace points of interest, e.g., bifurcation points, in parameter space [KRA07]. The advantage of this approach is that bifurcations of both stable and unstable solutions can be found, and often more efficiently and accurate than a sampling of the parameter space. A thorough description of the bifurcations then gives a complete and consistent picture of the qualitative dynamics that can occur when the control parameters are varied.

For the optically injected quantum-dot laser, the path-continuation along with an asymptotic analysis of the saddle-node and Hopf bifurcation lines has been performed for a model that incorporates the complex charge-carrier dynamics considered here, but which uses an $\alpha$-factor [OTT14]. Here, we will apply the path continuation on our model that dynamically calculates the carrier-induced index changes.

For the application of the path continuation tools it is advantageous to use a model that is as simple as possible in order to reduce the computational effort. A Thorough investigation of quantum-dot laser dynamics has been prevented by the high complexity of realistic models. In the following, we will therefore present a simplification of our full quantum-dot model, which we will then implement in the path continuation tools.

\subsubsection{Quantum-Dot Laser Model Simplification}

We will now reduce our quantum-dot laser model to a simpler form, which is easier to handle both numerically and analytically. In the process we have to take care not to neglect any of the important effects that define the unique features of quantum-dot laser dynamics.

Parts of this section have been published in [LIN14]. 
In Eq. (3.9) we had written the dynamics of the individual quantum-dot subgroups as

$$
\frac{\mathrm{d}}{\mathrm{d} t} \rho_{b, m}^{j}=-\operatorname{Re}\left(g_{m}^{j}\right)\left(\rho_{e, m}^{j}+\rho_{h, m}^{j}-1\right)|E|^{2}-W_{m} \rho_{e, m}^{j} \rho_{h, m}^{j}+S_{b, m}^{\mathrm{cap}} \pm \frac{S_{b}^{\mathrm{rel}}}{\nu_{m}},
$$

with the sign,+- accounting for the ground and excited-state dynamics, respectively. The dynamic equations for the charge-carrier occupations of different subgroups thus differ in the strength of the light-matter interaction with the laser field, given by the gain coefficient $g_{m}^{j}$. As we have seen before, the real part of the gain coefficient describes a Lorentzian lineshape around the resonance frequency $\omega$. The quantum-dots subgroups close to the resonance thus mostly dominate the light-matter interaction, whereas for sufficiently off-resonant subgroups the corresponding term can be neglected. A common approach [LUE10a, GIO12] is thus to separate the quantum-dots into two groups - active and inactive dots - and replacing the former smooth dependence of $\operatorname{Re}\left(g_{m}^{j}\right)$ on the quantum-dot frequencies with a discrete dependence: The complete stimulated emission takes place in the active dots, and vanishes in the inactive dots. In the following, we will describe the fraction of active dots with respect to the total quantum-dot number as $f^{\text {act }}$, and the corresponding fraction of inactive dots as $f^{\text {inact }}$.

We can write the dynamic equation for the two new subgroups, which we label with the indices "act" and "inact" for the active and inactive fractions of dots, respectively, as

$$
\begin{aligned}
\frac{\mathrm{d}}{\mathrm{d} t} \rho_{b, \mathrm{GS}}^{\mathrm{act}} & =\left.\frac{\partial \rho_{b, \mathrm{GS}}^{\mathrm{act}}}{\partial t}\right|_{\mathrm{stim}}-R_{\mathrm{sp}, \mathrm{GS}}^{\mathrm{act}}+S_{b, \mathrm{GS}}^{\mathrm{cap}, \mathrm{act}}+S_{b}^{\mathrm{rel}, \mathrm{act}} \\
\frac{\mathrm{d}}{\mathrm{d} t} \rho_{b, \mathrm{GS}}^{\text {inact }} & =-R_{\mathrm{sp}, \mathrm{GS}}^{\text {inact }}+S_{b, \mathrm{GS}}^{\mathrm{cap}, \text { inact }}+S_{b}^{\mathrm{rel} \text {,inact }} .
\end{aligned}
$$

Assuming now that the contribution of the quantum-dot excited states to the optical gain can be neglected, we can lump all excited states into one dynamic variable:

$$
\frac{\mathrm{d}}{\mathrm{d} t} \rho_{b, \mathrm{ES}}=-R_{\mathrm{sp}, \mathrm{ES}}+S_{b, \mathrm{ES}}^{\mathrm{cap}}-\frac{1}{2}\left[f^{\mathrm{act}} S_{b}^{\mathrm{rel}, \mathrm{act}}+f^{\mathrm{inact}} S_{b}^{\mathrm{rel}, \text { inact }}\right] .
$$

In above equations, the spontaneous recombination contribution is given by

$$
R_{\mathrm{sp}, \mathrm{GS}}^{(\mathrm{in}) \mathrm{act}}=W_{\mathrm{GS}} \rho_{\mathrm{GS}, e}^{(\mathrm{in}) \mathrm{act}} \rho_{\mathrm{GS}, h}^{(\mathrm{in}) \mathrm{act}}, \quad R_{\mathrm{sp}, \mathrm{ES}}=W_{\mathrm{ES}} \rho_{\mathrm{ES}, e} \rho_{\mathrm{ES}, h},
$$

and the charge-carrier scattering contributions

$$
\begin{aligned}
S_{b, m}^{\mathrm{cap},(\mathrm{in}) \mathrm{act}} & =S_{b, m}^{\mathrm{cap}, \mathrm{in}}\left[1-\rho_{b, m}^{(\mathrm{in}) \text { act }}\right]-S_{b, m}^{\mathrm{cap}, \text { out }} \rho_{b, m}^{(\mathrm{in}) \text { act }}, \\
S_{b}^{\text {rel,(in)act }} & =S_{b}^{\text {rel,in }}\left[1-\rho_{b, \mathrm{GS}}^{\text {(in)act }}\right] \rho_{b, \mathrm{ES}}-S_{b}^{\mathrm{rel}, \text { out }} \rho_{b, \mathrm{GS}}^{(\mathrm{in}) \text { act }}\left[1-\rho_{b, \mathrm{ES}}^{j}\right] .
\end{aligned}
$$


Next we will rescale the electric field such that

$$
|\hat{E}|^{2}:=N_{\mathrm{ph}},
$$

where $N_{\text {ph }}$ is the $2 \mathrm{D}$ photon density inside the laser cavity. The new electric field variable is thus related to the original field strength via

$$
\hat{E}=\sqrt{\frac{\varepsilon_{\mathrm{bg}} \varepsilon_{0}}{2 \hbar \omega} \frac{a_{L} h^{\mathrm{QW}}}{\Gamma}} E,
$$

where $a_{L} h^{\mathrm{QW}} / \Gamma$ is the effective mode height. The dynamic equation of the new electric field variable (without spontaneous emission) is thus given by

$$
\frac{\mathrm{d}}{\mathrm{d} t} \hat{E}(t)=(g(t)-\kappa) \hat{E} .
$$

We will now have to derive expressions for the complex optical gain $g(t)$. In Eq. (3.13) we had written

$$
g(t)=\frac{\hbar \omega \Gamma}{\varepsilon_{0} \varepsilon_{\mathrm{bg}} h^{\mathrm{QW}}} 2 N^{\mathrm{QD}} \sum_{j, m} \nu_{m} f(j) g_{m}^{j}\left(\rho_{e, m}^{j}+\rho_{h, m}^{j}-1\right)-i \delta \omega_{\mathrm{QW}} .
$$

We now rewrite the contribution of the quantum-dot states in terms of the active and inactive quantum-dot fractions as

$$
\left.g(t)\right|_{\mathrm{QD}}=\frac{\hbar \omega \Gamma}{\varepsilon_{0} \varepsilon_{\mathrm{bg}} h^{\mathrm{QW}}} 2 N^{\mathrm{QD}}\left[f^{\mathrm{act}} g_{\mathrm{GS}}^{\mathrm{act}}\left(\rho_{e, \mathrm{GS}}^{\mathrm{act}}+\rho_{h, \mathrm{GS}}^{\mathrm{act}}-1\right)+g_{\mathrm{ES}}\left(\rho_{e, \mathrm{ES}}^{\mathrm{act}}+\rho_{h, \mathrm{ES}}^{\mathrm{act}}-1\right)\right],
$$

where the gain coefficients of the lumped states are calculated by averaging over the individual subgroups:

$$
\begin{aligned}
& g_{\mathrm{GS}}^{\mathrm{act}}=\operatorname{Re}\left[\sum_{j} f(j) g_{\mathrm{GS}}^{j}\right]=\sum_{j} f(j) \frac{T_{2}\left|\mu_{\mathrm{GS}}\right|^{2}}{2 \hbar^{2}}\left(\frac{1}{1+\left[T_{2}\left(\omega_{\mathrm{GS}}^{j}-\omega\right)\right]^{2}}\right) \\
& g_{\mathrm{ES}}=i \operatorname{Im}\left[\sum_{j} 2 f(j) g_{\mathrm{ES}}^{j}\right]=-i \sum_{j} 2 f(j) \frac{T_{2}\left|\mu_{\mathrm{ES}}\right|^{2}}{2 \hbar^{2}}\left(\frac{T_{2}\left(\omega_{\mathrm{ES}}^{j}-\omega\right)}{1+\left[T_{2}\left(\omega_{\mathrm{ES}}^{j}-\omega\right)\right]^{2}}\right) .
\end{aligned}
$$

In the above, we take into account only the real part of the ground-state contribution to the gain into account, as the imaginary parts of the gain coefficient cancel each other out for excitation resonant to the maximum of the inhomogeneously broadened ground-state distribution. In principle the asymmetric occupation of the ground-states leads to a stronger gain at the lower-energy side of the distri- 
bution and thus to an asymmetric gain spectrum with non-vanishing index-shift. However, we found the carrier-induced frequency shift by this asymmetry to be negligible in comparison with the contributions of the other transitions. For the excited-state transitions, we only take the imaginary part of their gain coefficients into account, assuming that their contribution to the amplitude gain is negligible.

The contributions from the charge-carrier-reservoir transitions are written as

$$
\begin{aligned}
\delta \omega_{\mathrm{QW}} & =\frac{\hbar \omega \Gamma}{\varepsilon_{0} \varepsilon_{\mathrm{bg}} h^{\mathrm{QW}}} \frac{2}{A_{\mathrm{act}}} \sum_{\boldsymbol{k}^{2 \mathrm{D}}} \operatorname{Im} g_{k}^{2 \mathrm{D}}\left(f\left(\varepsilon_{e, k}^{2 \mathrm{D}}, E_{\mathrm{F}, e}^{\mathrm{eq}}, T^{\mathrm{eq}}\right)+f\left(\varepsilon_{h, k}^{2 \mathrm{D}}, E_{\mathrm{F}, h}^{\mathrm{eq}}, T^{\mathrm{eq}}\right)-1\right) \\
& \approx \delta \omega_{\mathrm{QW}}^{e} w_{e}+\delta \omega_{\mathrm{QW}}^{h} w_{h}+\delta \omega_{\mathrm{QW}}^{0}
\end{aligned}
$$

with

$$
\delta \omega_{\mathrm{QW}}^{0}=-\frac{\hbar \omega \Gamma}{\varepsilon_{0} \varepsilon_{\mathrm{bg}} h^{\mathrm{QW}}} \frac{2}{A_{\mathrm{act}}} \sum_{\boldsymbol{k}^{2 \mathrm{D}}} \operatorname{Im} g_{\boldsymbol{k}}^{2 \mathrm{D}} .
$$

In the above, we have approximated the sum over all individual $\boldsymbol{k}$-states by a linear dependence on the summed up reservoir densities $w_{b}$. For the densities typically encountered during the laser operation we have verified this to be a good approximation. The term $\delta \omega_{\mathrm{QW}}^{0}$ describes the constant frequency shift due to the absorption by the empty reservoir states. As a constant, we can compensate its effect by a proper choice of a rotating frame. In the following, we will therefore neglect this contribution.

We define

$$
\begin{aligned}
g_{\mathrm{GS}} & :=\frac{\hbar \omega \Gamma}{\varepsilon_{0} \varepsilon_{\mathrm{bg}} h^{\mathrm{QW}}} 2 N^{\mathrm{QD}} g_{\mathrm{GS}}^{\mathrm{act}}, \\
\delta \omega_{\mathrm{ES}} & :=-\operatorname{Im}\left[\frac{\hbar \omega \Gamma}{\varepsilon_{0} \varepsilon_{\mathrm{bg}} h^{\mathrm{QW}}} 2 N^{\mathrm{QD}} g_{\mathrm{ES}}\right],
\end{aligned}
$$

which allows us to write the complex optical gain in a very simple form:

$$
g(t)=g_{\mathrm{GS}}\left(\rho_{e, \mathrm{GS}}^{\mathrm{act}}+\rho_{h, \mathrm{GS}}^{\mathrm{act}}-1\right)-i \delta \omega_{\mathrm{ES}}\left(\rho_{e, \mathrm{ES}}^{\mathrm{act}}+\rho_{h, \mathrm{ES}}^{\mathrm{act}}\right)-i \delta \omega_{\mathrm{QW}}^{e} w_{e}-i \delta \omega_{\mathrm{QW}}^{h} w_{h} .
$$


With this we can now write the resulting rate-equation-system as

$$
\begin{aligned}
\frac{\mathrm{d}}{\mathrm{d} t} \hat{E}(t) & =(g(t)-\kappa) \hat{E} \\
\frac{\mathrm{d}}{\mathrm{d} t} \rho_{b, \mathrm{GS}}^{\text {act }} & =-\frac{g_{\mathrm{GS}}\left(\rho_{e, \mathrm{GS}}^{\mathrm{act}}+\rho_{h, \mathrm{GS}}^{\mathrm{act}}-1\right)}{f^{\mathrm{act}} a_{L} N^{\mathrm{QD}}}|\hat{E}|^{2}-R_{\mathrm{sp}, \mathrm{GS}}^{\mathrm{act}}+S_{b, \mathrm{GS}}^{\mathrm{cap}, \text { act }}+S_{b}^{\mathrm{rel}, \text { act }} \\
\frac{\mathrm{d}}{\mathrm{d} t} \rho_{b, \mathrm{GS}}^{\text {inact }} & =-R_{\mathrm{sp}, \mathrm{GS}}^{\text {inact }}+S_{b, \mathrm{GS}}^{\mathrm{cap}, \text { inact }}+S_{b}^{\mathrm{rel}, \text { inact }} \\
\frac{\mathrm{d}}{\mathrm{d} t} \rho_{b, \mathrm{ES}} & =-R_{\mathrm{sp}, \mathrm{ES}}+S_{b, \mathrm{ES}}^{\mathrm{cap}}-\frac{1}{2}\left[f^{\text {act }} S_{b}^{\text {rel,act }}+f^{\text {inact }} S_{b}^{\text {rel,inact }}\right] \\
\frac{\mathrm{d}}{\mathrm{d} t} w_{b} & =\frac{J}{e_{0}}-B^{S} w_{e} w_{h}-2 N^{\mathrm{QD}}\left[f^{\text {act }} S_{b, \mathrm{GS}}^{\mathrm{cap}, \text { act }}+f^{\text {inact }} S_{b, \mathrm{GS}}^{\mathrm{cap}, \text { inact }}+2 S_{b, \mathrm{ES}}^{\mathrm{cap}}\right] .
\end{aligned}
$$

\section{Fit Functions for Scattering Rates}

The above equation system has reduced the quantum-dot model to a 10 -variable rate-equation system. The charge-carrier occupations are, however, still coupled by the scattering rates, with complex dependencies on the reservoir charge-carrier densities. In the following, we therefore approximate these terms by easier fit functions, with only a few parameters to describe their dynamic dependencies.

A first look at the scattering rates in dependence of the reservoir densities $w_{b}$ reveals for the capture rates a quadratic increase at low densities, and a transition to nearly linear increase at higher values of $w_{b}$. The relaxation rates, on the other hand, show a linear increase at first and a subsequent saturation. This is depicted in Fig. 3.35 (solid lines). Taking these characteristics into account, we fit the scattering rates using the following functions

$$
\begin{aligned}
S_{b, m}^{\mathrm{cap}, \mathrm{in}}\left(w_{b}\right) & =\frac{A w_{b}^{2}}{B+w_{b}} \\
S_{b, m}^{\mathrm{rel}, \mathrm{in}}\left(w_{b}\right) & =\frac{C w_{b}}{D+w_{b}} .
\end{aligned}
$$

Table 3.3.: Fitting parameters for the shallow-dot scattering rates.

\begin{tabular}{lcccc}
\hline \hline & \multicolumn{2}{c}{ electrons } & \multicolumn{2}{c}{ holes } \\
& GS & ES & GS & ES \\
\hline $\mathrm{A}\left(10^{-11} \mathrm{~cm}^{2} \mathrm{~ns}^{-1}\right)$ & 18.5 & 48.3 & 10.5 & 21.4 \\
$\mathrm{~B}\left(10^{11} \mathrm{~cm}^{-2}\right)$ & 1.9 & 0.48 & 5.3 & 1.8 \\
$\mathrm{C}\left(\mathrm{ns}^{-1}\right)$ & 1014 & \multicolumn{2}{c}{2272} \\
$\mathrm{D}\left(10^{11} \mathrm{~cm}^{-2}\right)$ & 1.4 & \multicolumn{2}{c}{2.3} \\
\hline \hline
\end{tabular}



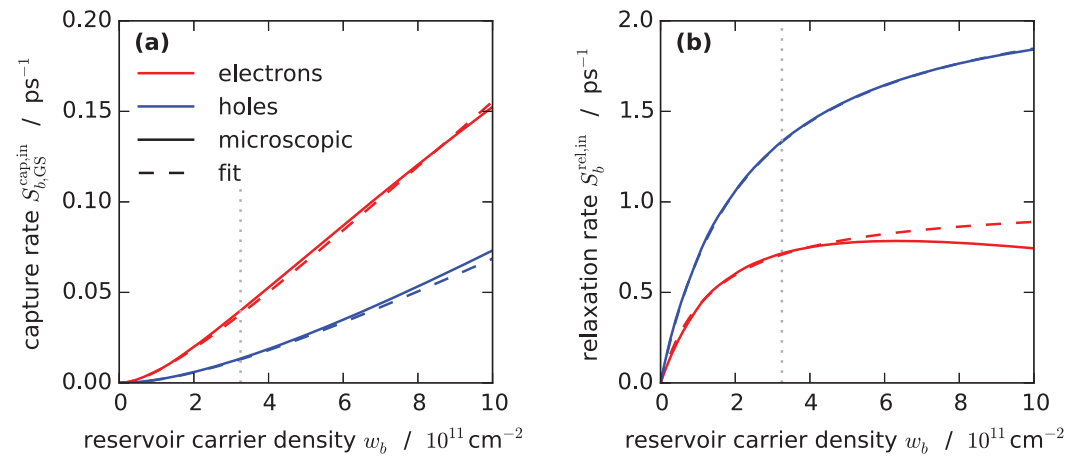

Figure 3.35.: Fits of the scattering rates for the shallow-dot device. Shown are (a) the direct-capture and (b) the intra-dot relaxation rate for electrons (red) and holes (blue), in dependence of the electron reservoir density $w_{e}$ (with $w_{h}=w_{e}, T=300 \mathrm{~K}$ ). The microscopically calculated rates (solid lines) are shown together with the simple fit functions (dashed lines). The dotted gray vertical lines show the value of $w_{e}$ at $J=2 J_{\mathrm{th}}$. Fit parameters are given in Table 3.3.

As we have seen before, the detailed balance relationship between the in and outscattering rates plays an important role for a correct description of the quantumdot laser dynamics. The corresponding out-scattering rates are therefore calculated from this condition, using Eqs. (2.22). The fitting parameters extracted from the microscopically calculated rates are given in Table 3.3 .

The comparison shown in Fig. 3.35 shows a good agreement between the microscopically calculated rates and the fit functions. For reservoir carrier densities above the typically encountered values (gray dotted vertical line for twice the threshold current) the fits show a slight deviation, especially pronounced in the electron relaxation rate. For not too high pump currents, however, the laser should be well in the region with good agreement. We will verify this by comparing the turn-on dynamics of the two approaches in the following.

\section{Comparison of the Full and Simplified Models}

We now verify the accuracy of our simplified 10-variable rate-equation model by comparing its results with the full quantum-dot model. The turn-on dynamics simulated with the two models is shown in Fig. 3.36. As we can see, our simplified approach reproduces the results of the full quantum-dot laser model excellently, with only slight differences in the reservoir carrier densities. In the following, we will therefore employ the simplified model to investigate the quantum-dot laser dynamics under optical injection. 

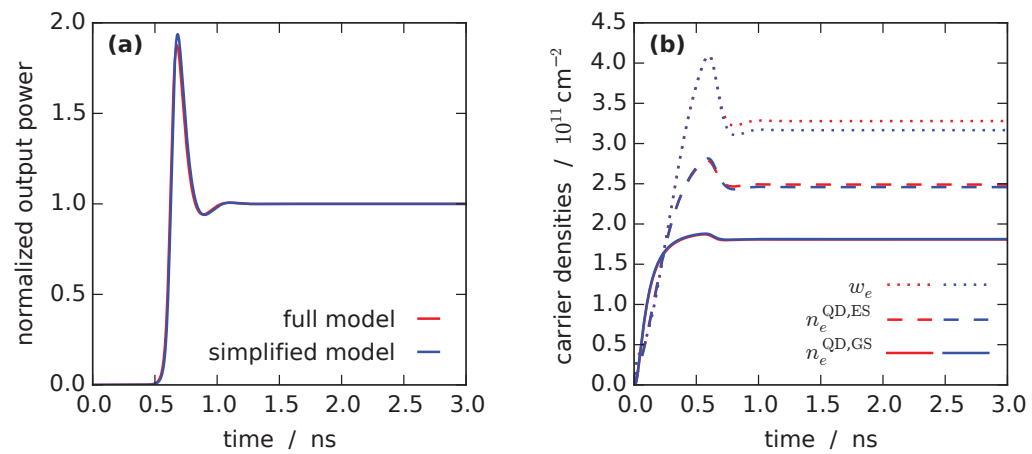

Figure 3.36.: Comparison of the full model (red lines) and the simplified 10-variable rate equation system (blue lines). Shown are the time-series of (a) the output power and (b) the electron densities during the turn-on of the shallow-dot laser. $J=2 J_{\mathrm{th}}$.

\subsubsection{Path Continuation Results}

We will now revisit the optically injected quantum-dot laser, using the simplified 10 -variable rate-equation model, Eqs. (3.100). The optical injection is implemented in the electric field equation in the same way as in Eq. (3.71), by moving into the rotating frame of the master laser:

$$
\frac{\mathrm{d}}{\mathrm{d} t} \hat{E}(t)=\left(g(t)-\kappa+i \delta \omega^{0}\right) \hat{E}+i 2 \pi \Delta \nu_{\mathrm{inj}} \hat{E}+K \kappa E^{0},
$$

where $\delta \omega^{0}=-\operatorname{Im} g^{0}$ is the frequency shift in the steady-state of the free-running laser, and $\Delta \nu_{\text {inj }}$ is the detuning of the master laser from the free-running laser frequency.

We use the path-continuation program AUT007p [DOE91, DOE09] to create bifurcation diagrams of the optically injected quantum-dot laser. The numerical parameters used in the simulations are given in Table 3.4. Starting with the shallow-dot laser, we show the bifurcation diagram in Fig. 3.37 a, depicting the results from the direct integration method combined with the bifurcations obtained from the path-continuation. Both approaches agree perfectly with each other, the numerically found boundaries of the phase-locked solutions match the bifurcations found by path-continuation. Additionally, we are now able to track the saddle-node and Hopf bifurcations beyond the phase-locked solution.

This reveals the underlying structure of the bifurcations of the phase-locked solution, as depicted in Fig. 3.37 b for a larger parameter region. The bifurcations to either side of the locking range can be seen to be connected. The parts of these bifurcations that apparently go through the locking region are bifurcations of 
Table 3.4.: Parameters used in the simplified 10 -variable rate-equation model for the shallow-dot laser.

\begin{tabular}{lll}
\hline \hline SYMBOL & VALUE & MEANING \\
\hline$N^{\mathrm{QD}}$ & $10^{11} \mathrm{~cm}^{-2}$ & QD density per layer \\
$a_{L}$ & 15 & number of layers \\
$B^{S}$ & $540 \mathrm{~nm}^{2} \mathrm{~ns}^{-1}$ & QW bimolecular recombination rate \\
$W_{\mathrm{GS}}$ & $0.44 \mathrm{~ns}^{-1}$ & GS spontaneous recombination rate \\
$W_{\mathrm{ES}}$ & $0.55 \mathrm{~ns}^{-1}$ & ES spontaneous recombination rate \\
$\kappa$ & $50 \mathrm{~ns}^{-1}$ & optical loss rate \\
$f^{\text {act }}$ & 0.5 & fraction of optically active quantum-dots \\
$g_{\mathrm{GS}}$ & $230 \mathrm{~ns}^{-1}$ & ground-state gain coefficient \\
$\delta \omega_{\mathrm{ES}}$ & $125 \mathrm{~ns}^{-1}$ & excited-state frequency-shift coefficient \\
$\delta \omega_{\mathrm{QW}}^{e}$ & $11.3 \times 10^{-11} \mathrm{~cm}^{2} \mathrm{~ns}^{-1}$ & reservoir electron frequency-shift coefficient \\
$\delta \omega_{\mathrm{QW}}^{h}$ & $5.5 \times 10^{-11} \mathrm{~cm}^{2} \mathrm{~ns}^{-1}$ & reservoir hole frequency-shift coefficient \\
\hline \hline
\end{tabular}

unstable fixed points, shown in light gray. By direct integration - or in experiments - these bifurcation could therefore not be found.

The period-doubling bifurcations of the oscillatory solutions outside of the locking range (up to period-4 oscillations) are shown in blue in Fig. 3.37 a. These also reveal a highly complex structure, reaching well inside the locking region. As we have discussed earlier, the two slim regions outside of the locking region around $K \approx 0.1$ with higher number of local extrema are not a consequence of perioddoubling bifurcations, but due to deformation of the periodic orbit.

While there is a multitude of additional bifurcations present, especially near the period-doubling regions, we will only calculate the bifurcations of the fixed points and period-doubling bifurcations up to period-4 oscillations. A complete discussion of all the bifurcations occurring in the system would be a task that far exceeds the scope of this work. In the following, we will therefore create bifurcation diagrams for different parameter sets. Furthermore, we will apply the path-continuation approach to our simplified quantum-dot model taking into account the carrierinduced frequency-shift from the off-resonant carriers. We will then compare these results to those obtained when using an $\alpha$-factor to describe the amplitude-phase coupling.

\section{Comparison with $\alpha$-Factor Approaches}

In order to discuss the impact of using a constant $\alpha$-factor, we additionally simulate the shallow-dot laser using the simplified model with the amplitude-phase coupling expressed by an $\alpha$-factor. We thus implement Eq. (3.75) to model the electric field dynamics. The simplified model yields $\alpha_{\mathrm{inj}}=1.42$, which differs slightly 
(a)

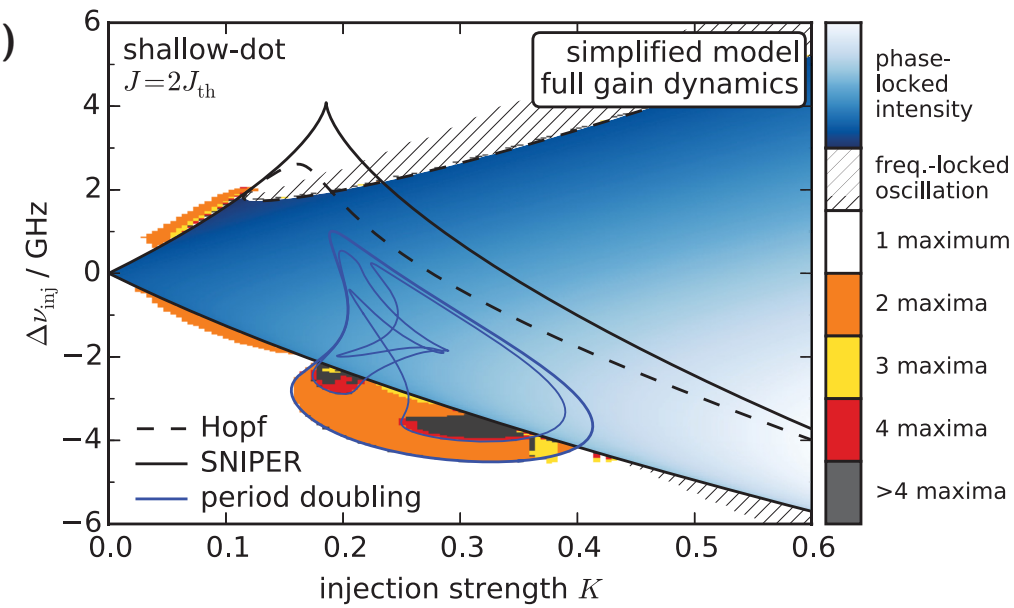

(b)

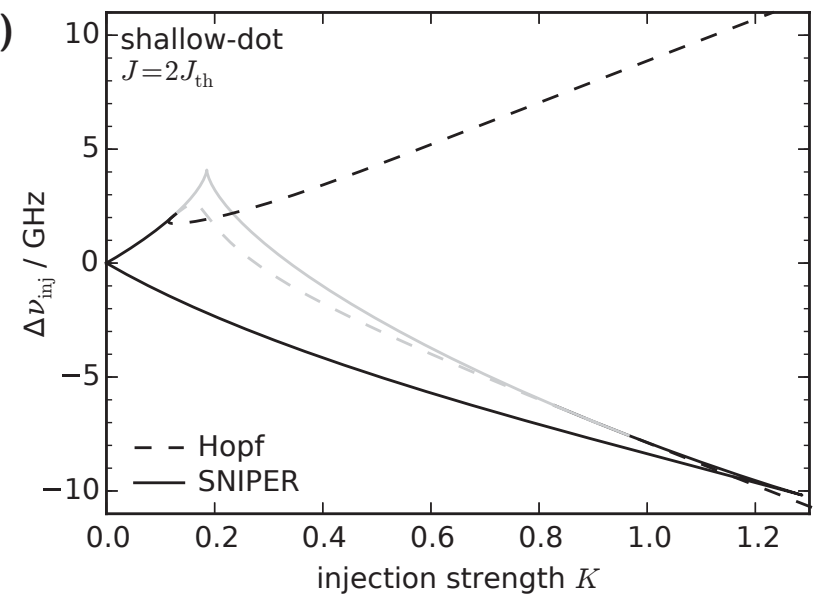

Figure 3.37.: Path-continuation results for the optically injected shallow-dot laser using the simplified quantum-dot laser model, Eqs. (3.100). (a) Shown are the laser dynamics obtained from direct integration, cf. Fig. 3.19, together with the bifurcation lines obtained using path continuation. Shown are SNIPER (black solid lines), Hopf (dashed lines) and period doubling (blue solid lines) bifurcations. (b) Bifurcation lines of the steady-state solution for a larger parameter area. Bifurcations of stable solutions are shown in black, those of unstable solutions in light gray. $J=2 J_{\mathrm{th}}$. Modified from [LIN14].

from the value obtained for the shallow-dot laser using the full model, due to the loss of spectrally resolved quantum-dot subgroups. The comparison between the bifurcation diagrams of the two approaches is shown in Fig. 3.38. As we had already seen using the full quantum-dot model, the two approaches yield very similar bifurcation lines for the phase-locked solution, but a shift of the bifurcations 

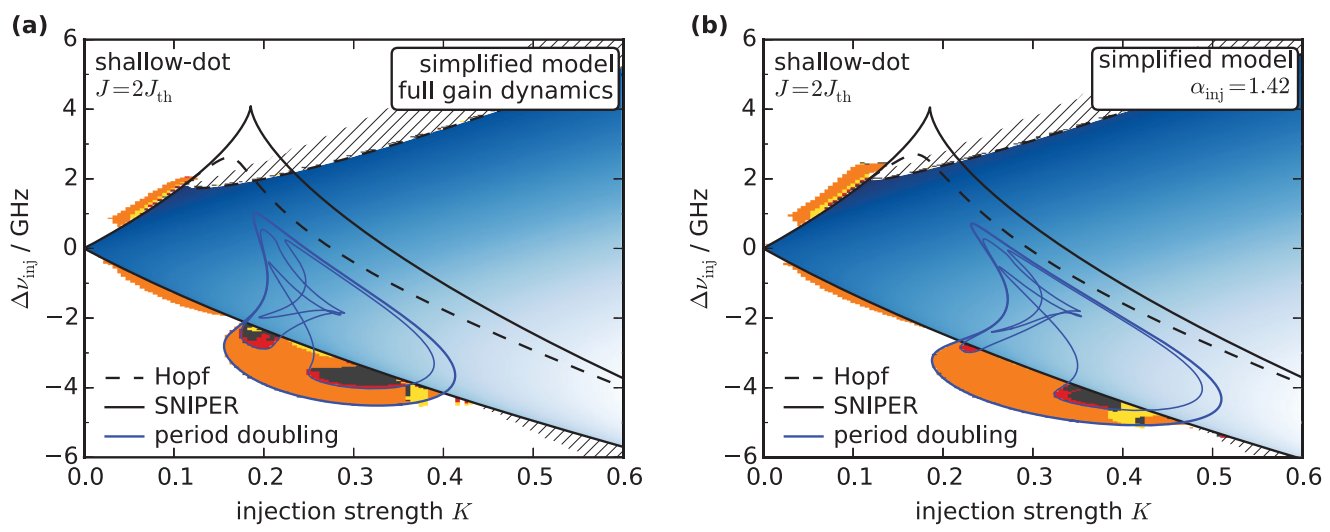

Figure 3.38.: Path-continuation results for the optically injected shallow-dot laser using the simplified quantum-dot laser model with (a) full gain-dynamics, Eqs. (3.100), and (b) using $\alpha_{\text {inj }}=1.42 . J=2 J_{\text {th }}$, cf. Fig. 3.37. Modified from [LIN14].

of the periodic orbits. The simplified model thus reproduces the results obtained with the full model very well.

\subsubsection{Dependencies on Scattering and Reservoir Loss Rates}

The simplified quantum-dot laser model is now applied to investigate the influence of the scattering rates and charge-carrier loss rates in the reservoir. The carrier-loss rates in the reservoir states are composed of non-radiative losses, e.g., recombination via lattice defects or Auger-recombination, and radiative recombination by spontaneous emission. Low reservoir losses are generally preferred, increasing the overall efficiency of the device. We will therefore exemplarily investigate the laser dynamics for reduced carrier loss rates. As we have written earlier, the scattering rates crucially depend on the quantum-dot material composition and size, as well as external parameters such as the temperature. As we have shown in Sec. 3.2.3, the quantum-dot laser operates in different dynamic regimes depending on the effective carrier scattering rate. The shallow-dot and deep-dot lasers we have discussed so far operate above the overdamped regime, within the synchronized regime. In the following we will therefore look at the case of reduced scattering rates, such that the laser is within the constant-reservoir regime.

Fig. 3.39 shows the dynamics of the optically injected shallow-dot laser device with its scattering rates reduced by a factor of 50 . This reduction makes the laser operate at the upper end of the constant-reservoir dynamic regime, with a strong desynchronization between the quantum-dot states and the reservoir. Immediately visible is the more symmetric shape of the locking region and the bifurcations of periodic solutions. Regions of period doubling bifurcations now exist at either side 

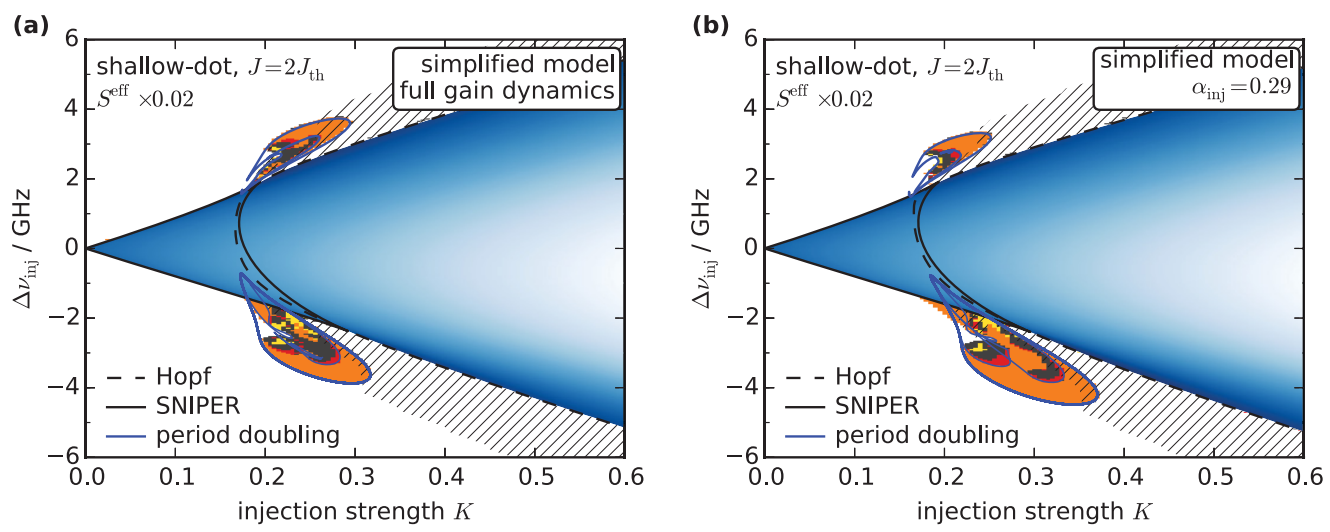

Figure 3.39.: Path-continuation results for the optically injected shallow-dot laser with scattering rates reduced by a factor 50 . Shown are results for the simplified quantumdot laser model with (a) full gain-dynamics and (b) using $\alpha_{\mathrm{inj}}=0.29 . \quad J=2 J_{\mathrm{th}}$, cf. Fig. 3.37. Modified from [LIN14].

of the locking region. This increased symmetry can be explained by a smaller amplitude-phase coupling due to the less effective coupling between resonant and off-resonant states, which also leads to a rather low value of $\alpha_{\text {inj }}=0.29$. Using this value in the model with a constant $\alpha$-factor leads to the bifurcation diagram shown in Fig. 3.39 b. Interestingly, the $\alpha$-factor approach leads to a much more asymmetric bifurcation structure. The bifurcations of the phase-locked region, on the other hand, again reveal a very good agreement.

The changes of the bifurcation structure introduced by the variation of the charge-carrier scattering rates again emphasizes the strong dependence of the amplitude-phase coupling on the internal charge-carrier dynamics. Even though the gain spectra of the lasers with full and reduced rates would be nearly identical, the response of the two lasers to the optical injection is very different.

We now take a look at the effects of reduced reservoir loss rates. When we decrease the loss rate to $B^{S}=54 \mathrm{~nm}^{2} \mathrm{~ns}^{-1}$, in addition to the reduced scattering rates, we obtain the bifurcation diagram shown in Fig. 3.40. Here, the modifications to the bifurcation diagram become much more pronounced. The bifurcations delimiting the phase-locking region become very asymmetric, whereas the dynamics in the unlocked region have a high degree of symmetry. When we simulate the quantum-dot laser using an $\alpha$-factor, the resulting bifurcation structure outside of the locking region shows a high degree of asymmetry. Here, the $\alpha$-factor approach clearly fails, as it can not reproduce the dynamics of the full modeling approach. 

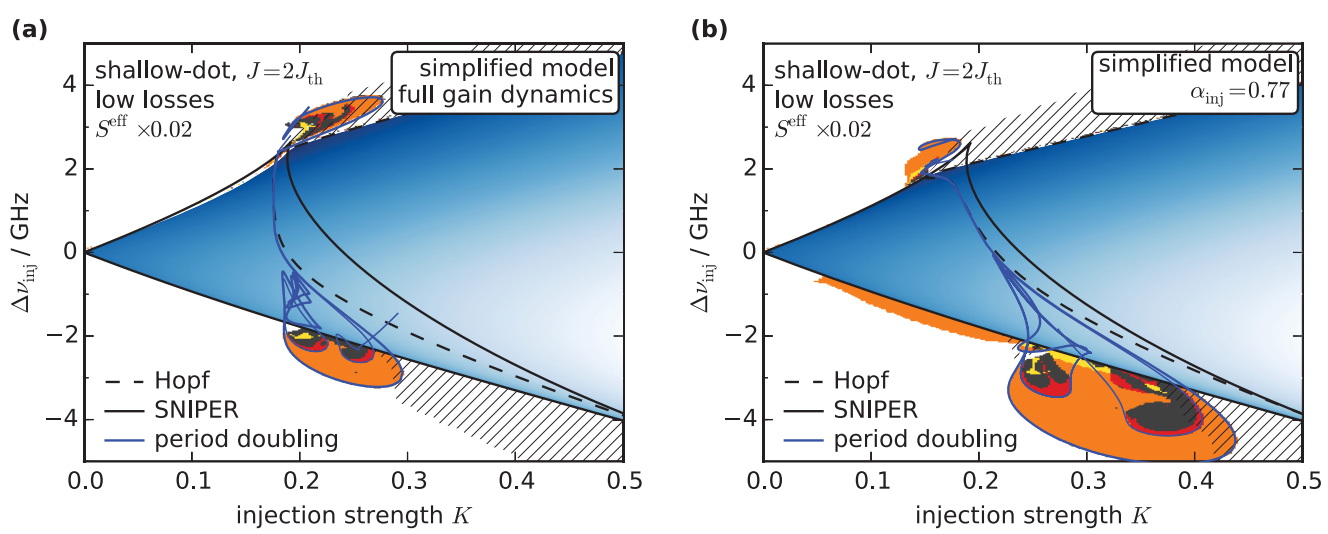

Figure 3.40.: Path-continuation results for the optically injected shallow-dot laser with scattering rates reduced by a factor 50, and reservoir losses reduced by a factor 10 .. Shown are results for the simplified quantum-dot laser model with (a) full gain-dynamics and (b) using $\alpha_{\mathrm{inj}}=0.77 . J=2 J_{\mathrm{th}}$, cf. Fig. 3.37. Modified from [LIN14].

\subsubsection{Summary}

To summarize the previous sections, we have found the optically injected quantumdot laser to exhibit dynamics that are qualitatively similar to conventional lasers, with a region of phase-locking to the master signal and regions of complex dynamics in the unlocked region. In general, the dynamics cannot be described by the use of an $\alpha$-factor, which is due to the desynchronized dynamics of the real and imaginary parts of the gain (and thus the optical susceptibility). While the bifurcations of the phase-locked solution with respect to the injection strength and detuning are well described by an $\alpha$-factor, the qualitative dynamics differ in the unlocked parameter range. This becomes especially pronounced for quantum-dot lasers that operate in the constant-reservoir regime, i.e., for slow scattering, and when reservoir-losses are low.

We have derived a simplified quantum-dot laser rate-equation model based on our microscopic modeling. The simplified model reproduces the dynamics of the full model very well, while being simple enough to be implemented in path-continuation packages. This opens up the possibility to perform further investigations of the quantum-dot laser dynamics by continuation of the occurring bifurcations, taking into account the unique features of quantum-dot lasers. 


\subsection{Dynamics under Optical Feedback}

In this section we will investigate the dynamics of quantum-dot lasers under the effect of time-delayed optical feedback. In contrast to the optical injection setup discussed in the previous sections, which required an external master signal, the perturbation of the laser under optical feedback is induced by its own light. The easiest way to realize optical feedback is to place a mirror in front of the laser diode in a distance $\ell$. The light coupled out of the laser cavity then reaches the cavity facet again after a time $\tau=\ell / v_{g}$, with the light group velocity $v_{g}$. The basic time-delayed optical feedback scheme is depicted in Fig. 3.41. The phasesensitive interference of the light inside the cavity with its time-delayed field can then lead to a variety of different dynamics [BES93, HEI01a, KAN05, SOR13, OTT14, KIM14]. Often, feedback effects are undesirable in applications where stable laser output is required, as feedback-induced periodic or even chaotic dynamics could appear [CHO84, HEN86, SCH881, TAR98a, OHT99]. On the other hand, optical feedback has been shown to improve device performance. For example, time-delayed feedback control has been implemented in laser devices to stabilize specific target states [AHL06a, SCH06a, DAH08b, SCH09a]. Furthermore, frequency stabilization [DAH87], noise suppression [SPA84, FLU07, MER09, LIN10e], and modulation bandwidth improvements [RAD07] by optical feedback has been shown. Deterministic chaos in semiconductor lasers has been utilized, e.g., for chaos communication [GOE98a, ABA01, TRO08, LUE11b, UCH12], or random-number generation [REI09a, OLI11]. In mode-locked lasers, optical feedback has been shown to substantially improve the phase-noise and timing-jitter [FIO11, OTT12a, ARS13, OTT14b].

The introduction of time-delay into the laser equation system mathematically increases the system's dimensionality to infinity. The state of the system at a given time $t$ is then given by the current values of the dynamical variables as well as by the values of the time-delayed variable over the whole interval $[t-\tau, t]$. The high dimension of the phase space then leads to the multitude of different dynamics that is observed in experiments and theory.

Parts of this section have been published in [LIN13].

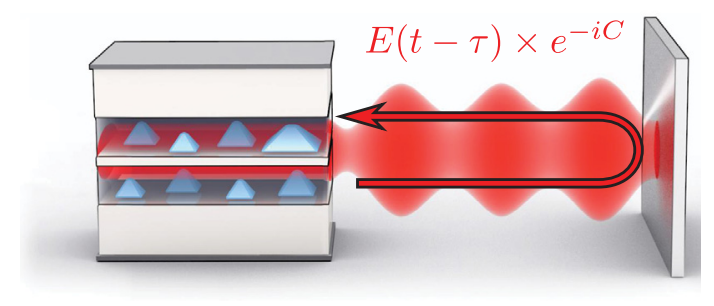

Figure 3.41: Sketch of the optical timedelayed feedback scheme. The outcoupled optical signal $E(t)$ is reflected off a mirror and reaches the cavity facet again after a time $\tau$, with a possible phase-shift $C$. The optical interaction within the laser cavity can lead to dynamic solutions. 
Recently, this high dimensionality of lasers with delayed optical feedback has been exploited for the implementation of optical neural networks [HIL02] and reservoir computing [PAQ12, DUP12, NGU14]. Here, the increase of dimensionality by the feedback leads to the formation of many "virtual network nodes", while only one physical laser node has to be employed, leading to an increase in computational power.

\subsubsection{Quantum-Dot Laser Model with Optical Feedback}

The theoretical implementation of the time-delayed optical feedback in the laser equations has been first realized in [ROZ75]. Lang and Kobayashi independently derived the corresponding theoretical formulation [LAN80b] in their seminal paper on feedback-induced effects in semiconductor lasers. The implementation of the feedback signal in the electric field equation is realized by a feedback term,

$$
\left.\frac{\partial}{\partial t} E(t)\right|_{\mathrm{fb}}=k_{\mathrm{fb}} e^{-i C} E(t-\tau)
$$

with a feedback rate $k_{\mathrm{fb}}$ and the feedback phase $C$. The appearance of the timedelayed variable $E(t-\tau)$ transforms the dynamic equations into a delay-differentialequation (DDE) system [ERN09]. In the above equation the phase shift $C$ enters due to the possibility of a phase difference between the laser field and the light reentering the laser cavity. This phase difference arises whenever the feedback length is not an integer multiple of the corresponding laser wavelength: $C=2 \pi \ell / \lambda^{0}=\tau / \omega^{0}$, with $\lambda^{0}$ and $\omega^{0}$ the wavelength and optical frequency of the free-running laser.

The above shows that delay time $\tau$ and feedback phase $C$ are coupled quantities, as both depend on $\ell$. However, a change of the feedback length by one laser wavelength leads to a rotation of the phase by $2 \pi$, while barely changing the delay time. The parameters $\tau$ and $C$ can therefore be seen as independent, and a characterization of the laser behavior under optical feedback needs to take into account the dependencies on both these parameters [HEI03a, ERZ07a]. This is in line with experimental difficulties of fine-adjusting and stabilizing the delay length on the resolution of a single laser wavelength, making the exact control of the feedback phase difficult.

We now transform our electric field into the rotating frame of the free-running laser, and rewrite the feedback rate as $k_{\mathrm{fb}} \equiv K_{\mathrm{fb}} \kappa$, with the new feedback strength $K_{\mathrm{fb}}$, similar to Eq. (3.71). Here, we will employ the full quantum-dot laser model, including microscopically calculated scattering rates, dynamic temperature and the multi-subgroup description. The electric field equations then become

$$
\frac{\mathrm{d}}{\mathrm{d} t} \widetilde{E}(t)=\left(g(t)-\kappa+i \omega^{0}\right) \widetilde{E}(t)+K_{\mathrm{fb}} \kappa e^{-i C} \widetilde{E}(t-\tau) .
$$


It must be noted that the parameter $C$ can only be interpreted as the phase difference between the cavity and the time-delayed field in the case of $\operatorname{Im} g(t)=-\omega^{0}$, i.e., in the free-running case. As soon as the feedback induces a frequency-shift away from the free-running laser frequency, the phase of the cavity field performs a rotation in the complex plane of $e^{\left[i\left(\operatorname{Im} g(t)+\omega^{0}\right) \tau\right]}$ during one delay time. The total phase-difference is then given by $C+\left(\operatorname{Im} g(t)+\omega^{0}\right) \tau$.

\subsubsection{Quantum-Dot Laser Dynamics under Optical Feedback}

In the following, we will discuss the bifurcations of the quantum-dot laser subject to time-delayed optical feedback. Quantum-dot lasers are known for their lower sensitivity to optical feedback when compared to conventional semiconductor laser devices [HUY04, OBR04, OTT10, GLO12]. This decreased sensitivity is commonly attributed to the higher relaxation-oscillation damping of quantum-dot lasers. This is supported by analytic calculations, showing that the critical feedback strength, at which the first bifurcations of cw-solutions appear, depends approximately linearly on the relaxation-oscillation damping [OTT12].

The dynamics of semiconductor lasers under time-delayed optical feedback can be understood in terms of external-cavity modes (ECMs). These refer to cw-solutions with constant frequency:

$$
\widetilde{E}(t)=\widetilde{E}^{s} e^{-i \delta \omega^{s} t},
$$

with a constant amplitude $\widetilde{E}^{s}$. The frequency deviation from the free-running laser is the given by $\delta \omega^{s}$. The external-cavity modes can be seen as standingwave solutions in the coupled laser-cavity and external-cavity system [LAN73, SHA85, ROS92c].

Inserting the ansatz Eq. (3.105) into Eq. (3.104) yields for the ECM frequency

$$
-\delta \omega^{s}=\left(\operatorname{Im} g^{s}+i \omega^{0}\right)+K_{\mathrm{fb}} \kappa \sin \left(\delta \omega^{s} \tau-C\right),
$$

which is a transcendental equation in $\delta \omega^{s}$, that also depends on the carrier-induced frequency-shift, $-\operatorname{Im} g^{s}$, which in turn depend on the charge-carrier dynamics of the quantum-dot system. From above equation it is possible to deduce that the number of $\delta \omega^{s}$ that fulfill the equation, i.e., the number of ECMs, increases with the feedback strength $K_{\mathrm{fb}}$. The ECMs always appear in pairs in saddle-node bifurcations, with one of them being always unstable [TAR95]. For high feedback strengths, a high number of ECMs coexist, which makes multi-stability a commonly encountered phenomenon [LEN91, HEI03a, FLU09, KIM14]. 
Figure 3.42: Numerically simulated bifurcation diagram in the feedback strength $K_{\mathrm{fb}}$ of the shallow-dot laser. Shown are the output power extrema in dependence of the feedback strength. The diagram was obtained by stepwise increase of $k_{\mathrm{fb}}$.

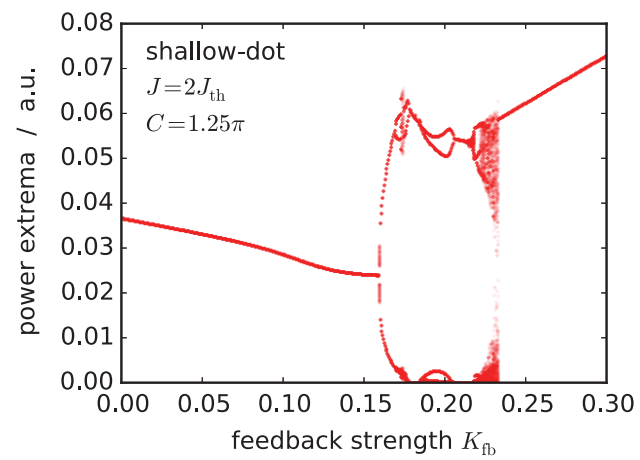

The stability of the quantum-dot laser can therefore be understood in terms of external-cavity modes. The occurring bifurcations characterize how the laser switches between different ECMs under changes of the feedback parameters [НОН99].

For the simulation of the quantum-dot laser with time-delayed optical feedback we choose a short delay time of $\tau=100 \mathrm{ps}$, corresponding to an external cavity length of $15 \mathrm{~mm}$. A numerical bifurcation diagram for a steady increase of the feedback strength is depicted in Fig. 3.42. At $K_{\mathrm{fb}} \approx 0.16$ the stability of the first ECM is lost in a Hopf bifurcation. The periodic orbit undergoes several bifurcations that lead up to a region of chaotic dynamics around $K_{\mathrm{fb}} \approx 0.23$, before the laser reaches the second stable ECM. This ECM remains stable for all higher feedback strengths. The bifurcation structure is qualitative similar to previous theoretical investigations of quantum-dot laser dynamics under feedback [OTT10, GLO12].

The chosen feedback length of $100 \mathrm{ps}$ lies within the short-cavity regime, where the laser response is known to sensitively depend on the feedback phase $C$ [HEI01a]. In order to get a complete picture of the dynamics, we numerically calculate bifurcation diagrams of the laser dynamics spanning the $\left(K_{\mathrm{fb}}, C\right)$-plane.

Fig. 3.43 shows the resulting bifurcation diagrams. In the regions denoted by the continuous color-code (dark blue to light blue) the laser is on a stable cw-solution, i.e., ECM. Fig. 3.43 a,b show different sweep directions of the feedback phase $C$. In these, the phase was either increased (panel (a)) or decreased (panel (b)), using the old system state as the initial state of the simulation with the new parameter value. Together with sufficiently small parameter changes, this assures that one stays on a given solution as long as it remains stable. In the previous discussion of the quantum-dot laser dynamics under optical injection we had limited ourselves to one sweep direction, as there the regions of multi-stability are much smaller than with optical feedback.

The two sweep directions reveal a bistability between two ECMs around $K_{\mathrm{fb}} \approx$ $0.12, C \approx 1.25 \pi$. Here, two saddle-node bifurcation lines collide at a cusp point. 

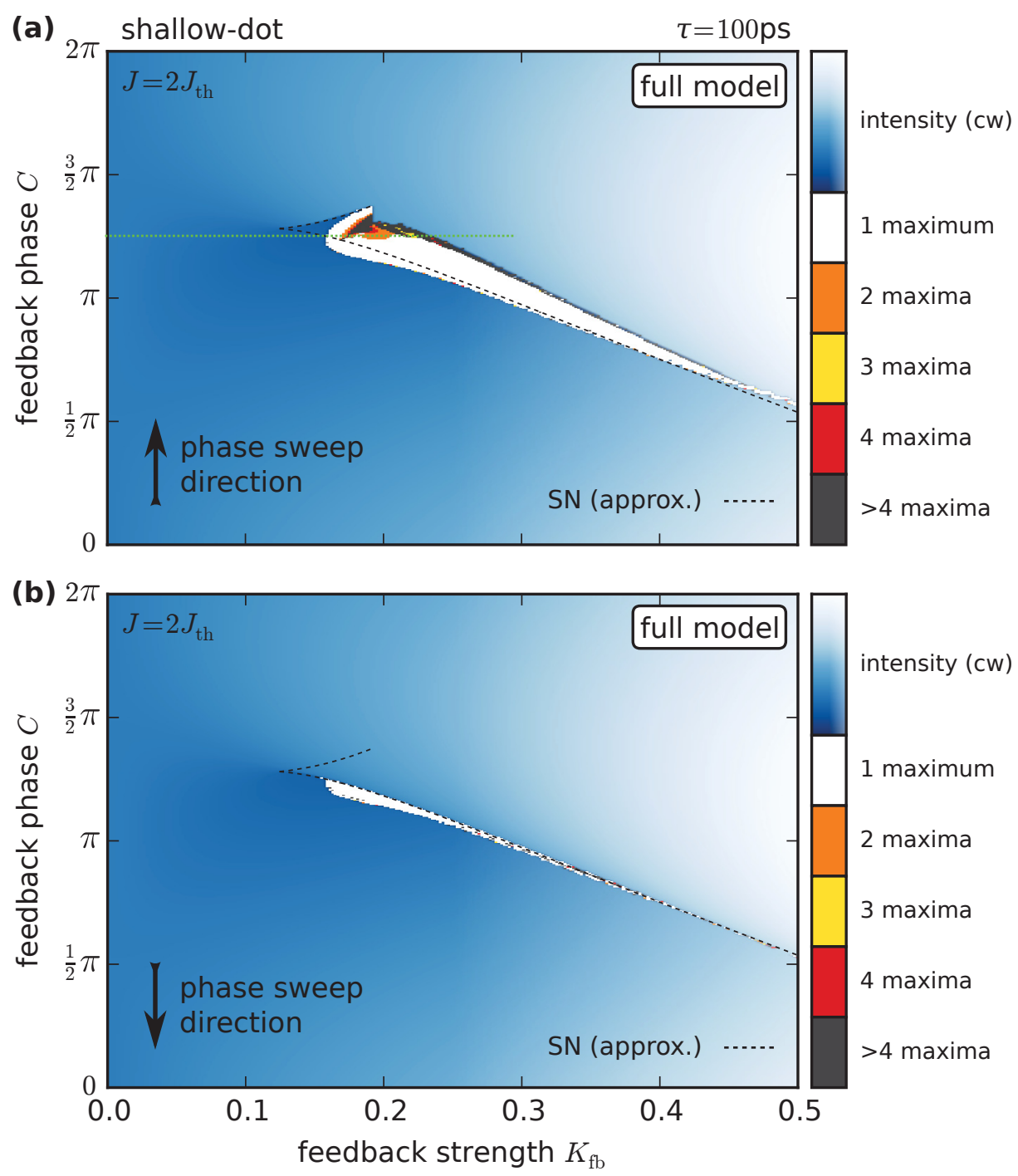

Figure 3.43.: Numerically simulated bifurcation diagram of the shallow-dot laser under optical feedback. Shown are the dynamics depending on the feedback strength $K_{\mathrm{fb}}$ and phase $C$. The continuous color code (dark blue to light blue) shows cw-solutions and their relative laser intensity. The discrete color code denotes oscillatory solutions. Shown are period-1 oscillations with one local maximum (white), two (orange), three (yellow) and four (red) maxima per oscillation period. Oscillations with higher number of maxima, an indication for chaotic and irregular oscillations, are shown by the dark gray color code. The dashed thin line denotes the numerically retrieved location of the saddle-node (SN) bifurcation. The arrow denotes the direction of the phase-sweep in (a) and (b). $J=2 J_{\mathrm{th}}$, cf. Fig. 3.19. The green dotted line in (a) shows the parameter range covered in Fig. 3.42. After [LIN13]. 


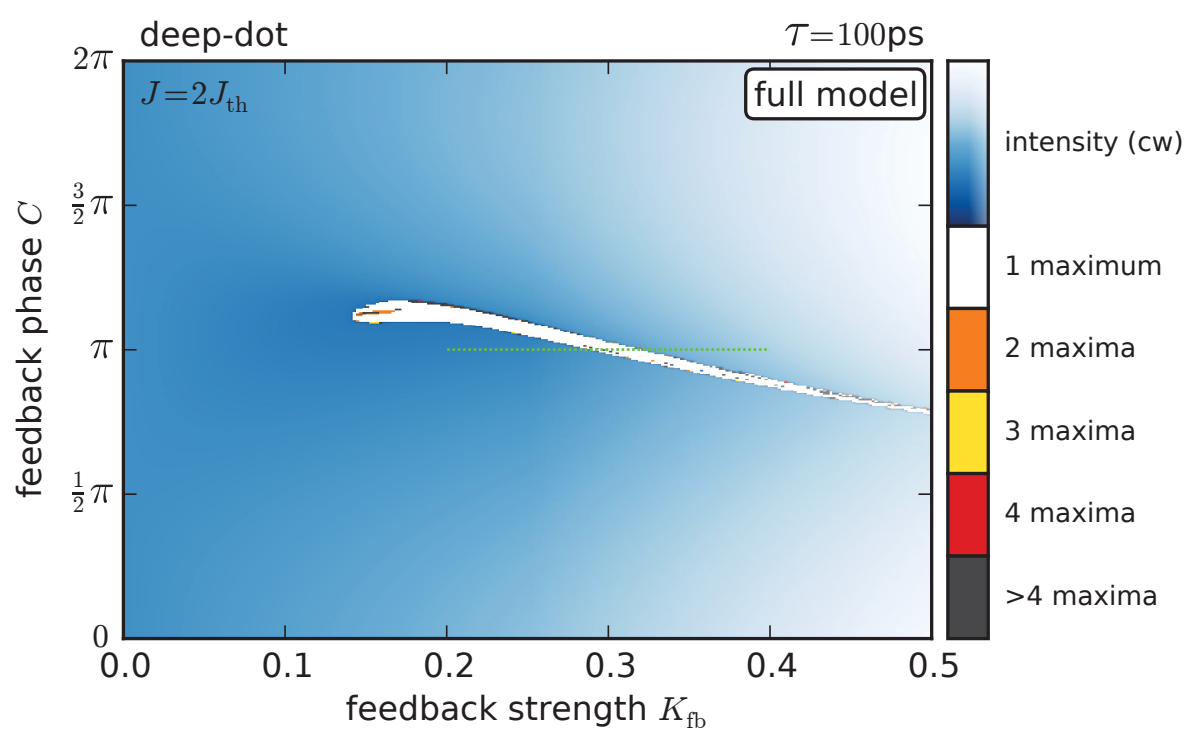

Figure 3.44.: Numerically simulated bifurcation diagram of the deep-dot laser under optical feedback. $J=2 J_{\text {th }}$, cf. Fig. 3.43. The dotted green line shows the parameter range covered in Fig. 3.45. After [LIN13].

This cusp marks the creation of the first additional ECM. Between the two saddlenode lines originating in this cusp point, the two ECMs coexist. We can distinguish the two solutions by their different intensities. The higher-intensity solution is followed in the parameter space for a downward sweep of the feedback phase (panel (b)), until it is destroyed in the saddle-node bifurcation.

The lower-intensity ECM, on the other hand, exhibits a variety of bifurcations, visible in Fig. 3.43 a. Slightly above the cusp point, in terms of $K_{\mathrm{fb}}$, this ECM undergoes a Hopf bifurcation, in which the ECM loses its stability and the laser dynamics is forced onto a stable periodic orbit. This is denoted by the white area in Fig. 3.43. This periodic solution undergoes additional bifurcations. Especially close to the cusp point, period-doubling bifurcations are visible, leading to periodic orbits of higher periodicity. This is shown by the increasing number of maxima, denoted by the color code in the bifurcation diagram. For $K \gtrsim 0.2$, the upper boundary of the periodic region is lined with a quasi-periodic region, born in a torus bifurcation of the periodic orbit. The torus becomes unstable when increasing $C$ even higher, and the laser reaches the higher-intensity ECM.

Next, we simulate the deep-dot laser with optical feedback. The resulting bifurcation diagram is shown in Fig. 3.44. Compared to the shallow-dot laser, the dynamics are now much more stable. While the first additional ECM is born at approximately the same $K_{\mathrm{fb}}$, the region of accompanying bifurcations is much smaller. Only a slim region of periodic oscillation is visible. Furthermore, this 


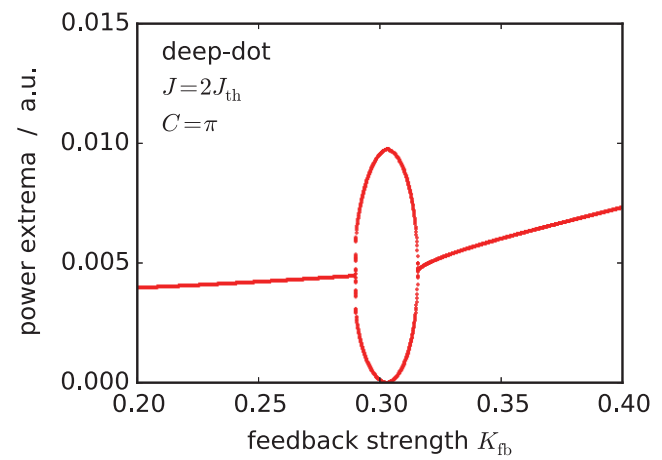

Figure 3.45: Numerically simulated bifurcation diagram in the feedback strength $K_{\mathrm{fb}}$ of the deep-dot laser. Shown are the output power extrema in dependence of the feedback strength. The diagram was obtained by stepwise increase of $k_{\mathrm{fb}}$.

region is delimited in large parts on both sides of the feedback phase by Hopf bifurcations. Note, that the position of the saddle-node bifurcations in parameter space does not change under a variation of the effective charge-carrier scattering rates [GLO12]. Nevertheless, the different amplitude-phase coupling in the deep-dot structure leads to a shift of the saddle-node lines compared to the shallow-dot. The closeness of the saddle-node and Hopf bifurcations at the edges of the dynamic region, however, makes a numeric identification of the bifurcation lines difficult, and would require additional investigations. While possible [GLO12], the path continuation of bifurcations in delay-differential equation systems is much more complex, and will thus not be part of this work.

Nevertheless, we can identify a qualitative difference in the way the ECMs are organized due to the changes in bifurcation structure. The two ECMs are now connected by a periodic orbit born in the Hopf bifurcations, leading to a bridge of periodic dynamics between the steady-state ECM solutions [PIE01]. This is illustrated in Fig. 3.45.

\section{Comparison with $\alpha$-Factor-Based Models}

We now proceed in terms of a comparison of the quantum-dot laser dynamics under optical feedback with the conventional approach of using an $\alpha$-factor for describing the amplitude-phase coupling, analogous to the case under optical injection in the previous sections. We thus rewrite the electric field equation in terms of an $\alpha_{\mathrm{fb}}$ :

$$
\frac{\mathrm{d}}{\mathrm{d} t} \widetilde{E}(t)=(\operatorname{Re} g(t)-\kappa)\left(1-i \alpha_{\mathrm{fb}}\right) \widetilde{E}(t)+K_{\mathrm{fb}} \kappa e^{-i C} \widetilde{E}(t-\tau) .
$$

The value of $\alpha_{\mathrm{fb}}$ is evaluated from the laser response to an infinitesimal feedback rate.

$$
\alpha_{\mathrm{fb}}:=-\frac{\partial \operatorname{Im} g^{s} / \partial K_{\mathrm{fb}}}{\partial \operatorname{Re} g^{s} / \partial K_{\mathrm{fb}}}=-\frac{\left.\operatorname{Im} g^{s}\right|_{K_{\mathrm{fb}}=\Delta K}-\left.\operatorname{Im} g^{s}\right|_{K_{\mathrm{fb}}=0}}{\left.\operatorname{Re} g^{s}\right|_{K_{\mathrm{fb}}=\Delta K}-\left.\operatorname{Re} g^{s}\right|_{K_{\mathrm{fb}}=0}} .
$$



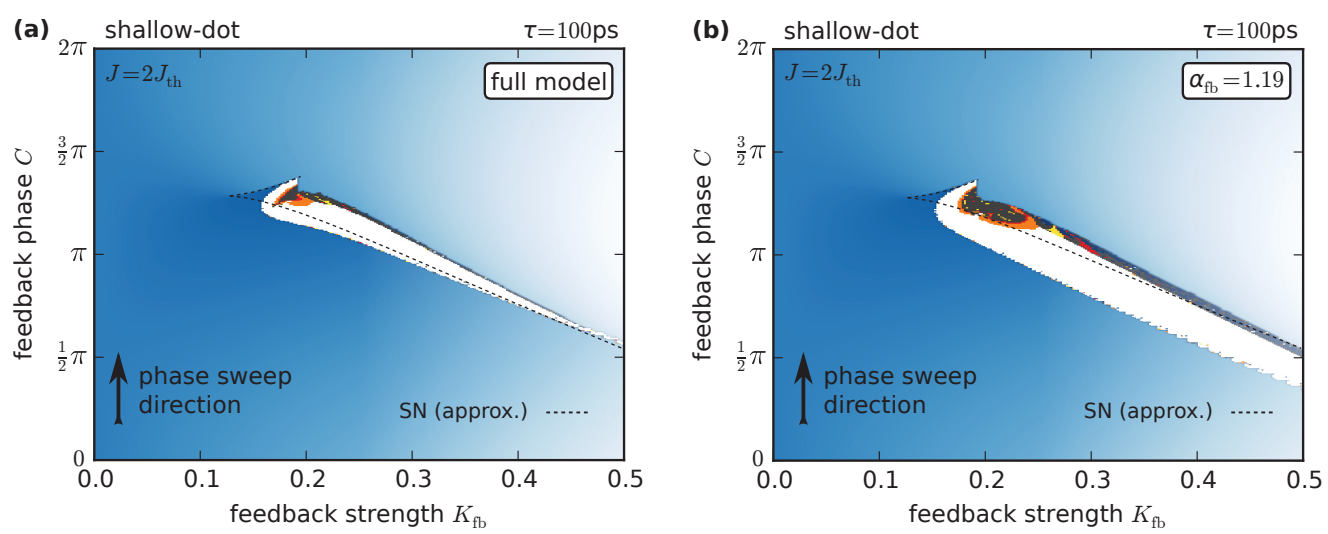

Figure 3.46.: Comparison of (a) the full model, Eq. (3.104), and (b) the $\alpha$-factor approach, Eq. (3.107) of the shallow-dot laser dynamics under optical feedback. $J=2 J_{\mathrm{th}}$, cf. Fig. 3.43. After [LIN13].

As in the case of optical injection, we again evaluate the adiabatic change of the steady-state value of the complex gain, $g^{s}$, under a change of the perturbation parameter. Comparing Eqs. (3.108) and (3.81) it becomes clear that in fact $\alpha_{\text {inj }}=$ $\alpha_{\mathrm{fb}}$ holds, as long as the laser reaches a steady-state. This can be understood intuitively by the origin of the perturbation that induces the gain and frequencyshift variations. In both cases an increased optical field leads to a higher stimulated recombination rate that, in turn, induces a variation of the carrier distribution, which then changes the complex optical gain $g^{s}$. We can thus use the values obtained for $\alpha_{\text {inj }}$ in the simulations of the optical injection in the optical feedback simulations.

For the shallow-dot laser at twice the threshold current the bifurcation diagrams under optical feedback are shown in Fig. 3.46 for the full modeling approach, Eq. (3.104), and using an $\alpha$-factor, Eq. (3.107). The comparison between the two diagrams reveals a similar location of the saddle-node bifurcation lines of the first ECM between the two approaches. When describing the amplitude-phase coupling with $\alpha_{\mathrm{fb}}$, however, the extent of the periodic regions in parameter space is greatly exaggerated. The Hopf bifurcation on the lower boundary of the oscillatory region is shifted towards lower values of the feedback phase $C$, resulting in a broader region of stable periodic oscillations. Furthermore, the regions of complex dynamics near its upper boundary is enlarged as well.

This behavior is similar to what we had observed in the case of optical injection. The saddle-node lines, which are bifurcations involving exclusively fixed points, are well described in both approaches. As soon as dynamic solutions play a role, e.g., Hopf or period doubling bifurcations, the $\alpha$-factor approach becomes inaccurate. 

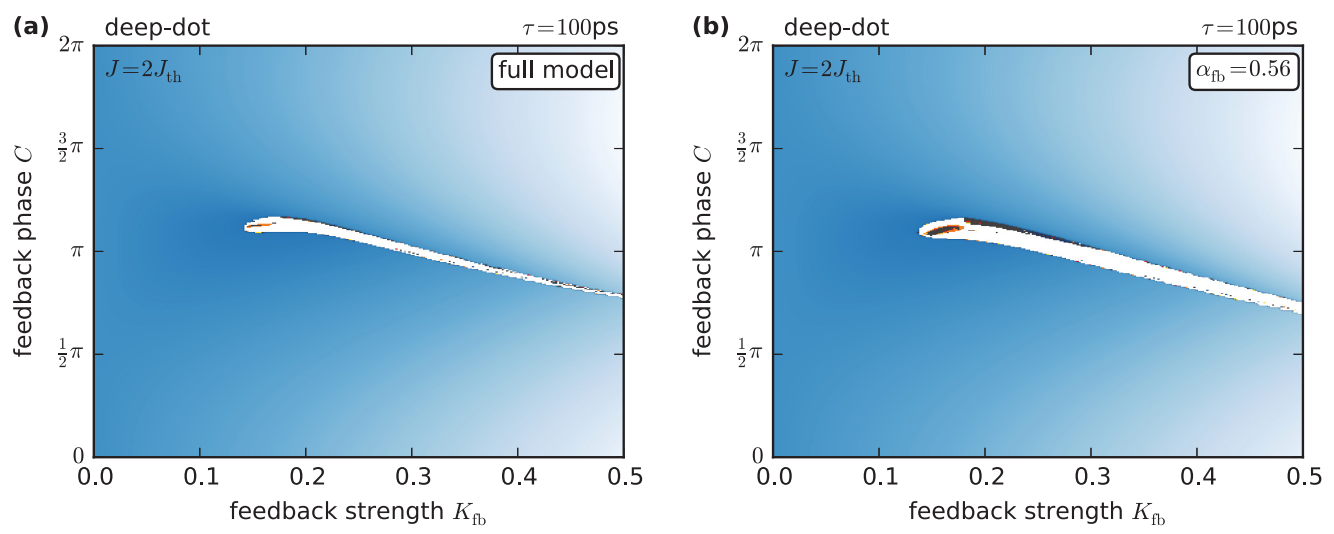

Figure 3.47.: Comparison of (a) the full model, Eq. (3.104) and (b) the $\alpha$-factor approach, Eq. (3.107) of the deep-dot laser dynamics under optical feedback. $J=2 J_{\mathrm{th}}$, cf. Fig. 3.43. After [LIN13].

In the optical feedback setup this leads to a prediction of lower stability towards optical feedback, due to larger areas of periodic and irregular dynamics.

The bifurcation diagrams for the deep-dot laser show the same trend, as depicted in Fig. 3.47. The $\alpha$-factor approach again predicts a larger area of complex dynamics than the full model. Due to the smaller periodic region in the deep quantum-dot laser this effect is less pronounced than previously, but nevertheless a clear destabilization of the laser dynamics can be observed.

The results presented here lead to an important conclusion about quantum-dot laser stability. The strong damping of the relaxation oscillations in quantum-dot lasers does explain their higher stability to optical perturbations only in part. The dynamics of the amplitude-phase coupling also lead to a higher dynamical stability by reducing the extent of complex dynamics in parameter space. This again shows that the $\alpha$-factor should not be used when an accurate prediction of the quantumdot laser dynamics is required. 


\subsection{Small-Signal Frequency Response of Quantum-Dot Lasers}

In the previous sections we have shown that the amplitude-phase coupling in quantum-dot lasers can be quite different than in conventional quantum-well or bulk laser diodes. The modeling of the dynamical response to optical perturbations therefore requires an approach that goes beyond a simple $\alpha$-factor to describe the carrier-induced index changes and thus the frequency modulation. The frequency modulation also plays an important role in modulated lasers. For example under direct pump current modulation, the variation of the pump current will not only induce a gain change and thus an amplitude modulation, but also a change in the optical frequency. This induces a so-called frequency chirp, which leads to sidebands in the optical spectrum. A low frequency chirp is thus generally preferred in amplitude-modulated signals.

\subsubsection{Evaluation of the Frequency and Amplitude Modulation Indices}

In the following we will apply the simplified quantum-dot laser model for the shallow-dots derived in Sec. 3.7.1. Under small-signal modulation of the laser device with a modulation frequency $f$, we can assume a harmonic response of the complex optical gain $g(t)$ around its steady-state value $g^{s}$. We can thus write

$$
g(t)=g^{s}+\Delta g^{\prime} \cos \left(2 \pi f t+\phi^{\prime}\right)+i \Delta g^{\prime \prime} \cos \left(2 \pi f t+\phi^{\prime \prime}\right),
$$

where $\Delta g^{\prime}, \Delta g^{\prime \prime}$ are the corresponding modulation amplitudes of the real and imaginary parts of the gain. We allow a phase shift in each of the individual parts of the response, given by $\phi^{\prime}, \phi^{\prime \prime}$. Inserting this into the electric field equation yields

$$
\frac{\mathrm{d}}{\mathrm{d} t} \hat{E}(t)=\left[\Delta g^{\prime} \cos \left(2 \pi f t+\phi^{\prime}\right)+i \Delta g^{\prime \prime} \cos \left(2 \pi f t+\phi^{\prime \prime}\right)\right] \hat{E}(t),
$$

where we have exploited the gain clamping condition, i.e., $g^{s}$ exactly balances out the optical losses in the steady-state.

The instantaneous frequency of the electric field is given by $\operatorname{Im} \frac{\mathrm{d} \hat{E}(t) / \mathrm{d} t}{\hat{E}(t)}$, which allows us to identify the frequency chirp, i.e., the modulation amplitude of the instantaneous frequency, as

$$
\Delta \omega=\Delta g^{\prime \prime}
$$

Parts of this section have been published in [LIN14]. 
and the so-called frequency modulation index, $\beta$, defined as

$$
\beta:=\frac{\Delta \omega}{2 \pi f}=\frac{\Delta g^{\prime \prime}}{2 \pi f} .
$$

The amplitude modulation can be calculated from the real part of the gain modulation. The electric field amplitude can thus be calculated from

$$
\frac{\mathrm{d}}{\mathrm{d} t}|\hat{E}(t)|=\Delta g^{\prime} \cos \left(2 \pi f t+\phi^{\prime}\right)|\hat{E}(t)|,
$$

which can be readily solved:

$$
|\hat{E}(t)|=\hat{E}^{0} \exp \left[\frac{\Delta g^{\prime}}{2 \pi f} \cos \left(2 \pi f t+\phi^{\prime}\right)\right],
$$

with the steady-state amplitude $\hat{E}^{0}$. Under small-signal modulation we can assume the gain modulation to be small, i.e., $\exp (\varepsilon) \approx 1+\varepsilon$ (for $\varepsilon \ll 1$ ). The above equation can then be used to calculate the so-called amplitude modulation index $m$, defined as

$$
m:=\frac{\Delta P}{P^{0}}=\frac{\max P(t)-\min P(t)}{2 P^{0}}=\frac{\Delta g^{\prime}}{\pi f} .
$$

The ratio of the frequency and amplitude modulation indices, $2 \beta / m$, then yields an experimentally accessible way of determining the relative gain modulation [HAR83]:

$$
\frac{2 \beta}{m}=\frac{\Delta g^{\prime \prime}}{\Delta g^{\prime}} .
$$

Such FM/AM measurements are an established procedure to determine the amplitudephase coupling of semiconductor laser devices. This becomes immediately clear if we express the complex gain by an $\alpha$-factor. Then, the simple relation

$$
\Delta g^{\prime \prime}=-\alpha \Delta g^{\prime}
$$

holds, and we immediately see that

$$
\frac{2 \beta}{m} \equiv \alpha
$$

\subsubsection{Numerical Evaluation of FM/AM Measurements}

We will now employ the FM/AM technique to determine the frequency-resolved amplitude-phase coupling in quantum-dot lasers. As we have seen in Eq. (3.118), if 

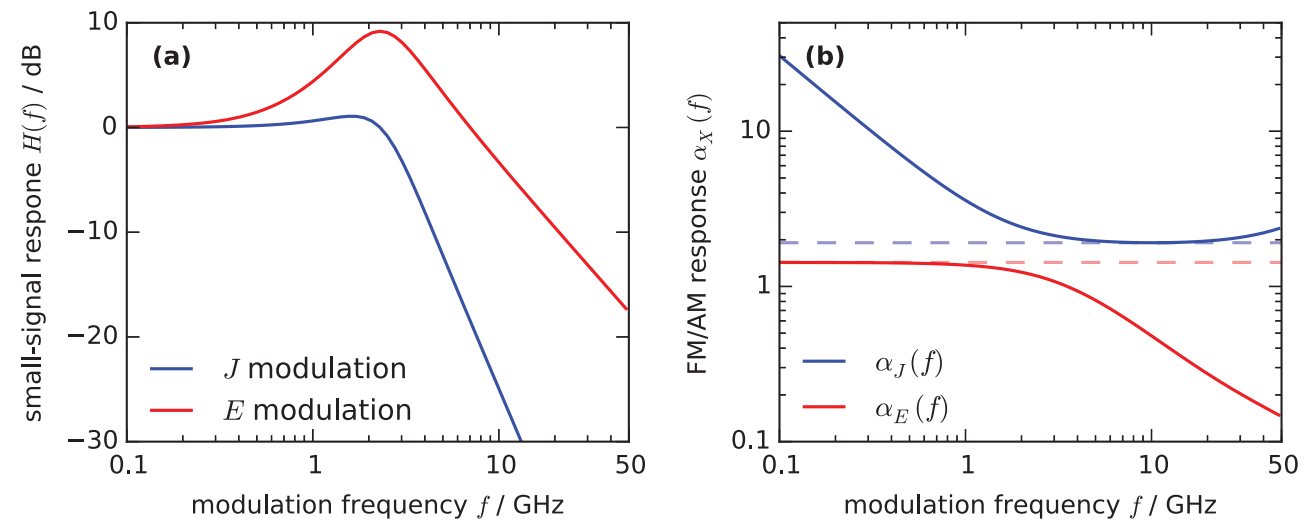

Figure 3.48.: Small-signal modulation response of the quantum-dot laser under current and electric field modulation. (a) Small-signal transfer function $H(f)$ (cf. Eq. (3.43)) for pump current (blue) and electric field modulation (red). (b) FM/AM response $\alpha_{X}(f)$, for $X=J$ (blue), $X=E$ (red) for current and electric field modulation, respectively. The dashed lines show $\min \left(\alpha_{J}\right)=1.91$ (blue) and $\max \left(\alpha_{E}\right)=1.42$ (red), respectively. Modified from [LIN14].

the amplitude-phase coupling could be described by an $\alpha$-factor, we would expect a flat dependence of $2 \beta / \mathrm{m}$ on the modulation frequency.

In the following, we will consider two different modulation techniques. The most simple is the direct modulation of the pump current, i.e., we set

$$
J(t)=J^{0}+\Delta J \cos 2 \pi f t,
$$

with the small pump-current modulation amplitude $\Delta J$. Additionally, we also consider a modulation of the cavity field, realized by a small harmonic source term in the electric field:

$$
\frac{\mathrm{d}}{\mathrm{d} t} \hat{E}(t)=(g(t)-\kappa) \hat{E}(t)+\Delta \hat{E} \cos 2 \pi f t,
$$

with the modulation amplitude $\Delta \hat{E}$. Following the argumentation leading to Eq. (3.118), we denote the FM/AM ratio in the two cases as $\alpha_{J}(f)$ and $\alpha_{E}(f)$ for the pump current and electric field modulation, respectively.

Fig. 3.48 a shows the small-signal response of the quantum-dot laser under the different types of modulation. The small-signal transfer function shows the damped peak and subsequent decay that we had already seen in Sec. 3.4. The corresponding frequency response, shown in Fig. $3.48 \mathrm{~b}$, reveals a very high value of $\alpha_{J}$ for small modulation frequencies, that decreases towards a plateau around $f=10 \mathrm{GHz}$. This high value at low modulation frequencies is also known from conventional 
laser devices, and attributed to gain compression effects [COL12a]. This can be understood intuitively by the gain-clamping condition. The slower the modulation the closer the laser can follow this parameter change. In the limit of $f \rightarrow 0$ the laser would adiabatically follow the modulation and always assume a state where the gain-clamping condition is fulfilled, i.e. $g(t) \equiv g^{s}$, such that the real part of the gain modulation $\Delta g^{\prime}$ would vanish. Unless $\Delta g^{\prime \prime}=0$, the FM/AM ratio will thus tend to infinity for $f \rightarrow 0$. In order to extract a value for the $\alpha$-factor from the FM/AM measurement, one therefore evaluates the value of $2 \beta / m$ for high enough modulation frequency, where the dependence of $\alpha_{J}(f)$ on $f$ is nearly flat, i.e., its minimum value. In our case this yields $\min \left(\alpha_{J}\right)=1.91$, denoted by the dashed blue line in Fig. 3.48.

In contrast to conventional lasers, where the $\mathrm{FM} / \mathrm{AM}$ response remains flat for high enough modulation frequency, we can observe a rise of $\alpha_{J}$ for high frequencies around $f \gtrsim 20 \mathrm{GHz}$. This rise has been attributed to the charge-carrier scattering processes [MEL06], which influence the modulation response, when $f$ comes close to the involved scattering rates [WAN12a]. Then, the finite scattering rate restricts the transfer of the charge-carrier modulation to the ground-state occupation, reducing $\Delta g^{\prime}$ and thus increasing the relative frequency chirp. For conventional lasers, this effect would be visible only for frequencies close to the intra-band relaxation rates, which are much faster than the charge-carrier scattering rates in quantumdot lasers. This signature of the scattering processes in the frequency response of quantum-dot lasers has been experimentally observed [GER08]. In [MEL06] it has been proposed to use the minimal value of $\alpha_{E}(f)$ as the "result" of the FM/AM measurements, $\alpha_{\mathrm{FM} / \mathrm{AM}}$, which is a reasonable choice for directly modulated quantum-dot lasers.

We now also take a look on the modulation behavior under electric-field variation. The source term in Eq. (3.120) induces a modulation of the electric field that is transferred to the charge-carriers by a modulated stimulated recombination rate. The resulting amplitude modulation exhibits a strongly pronounced resonance peak at around $2.5 \mathrm{GHz}$. The corresponding FM/AM response shows a much different picture than under current modulation. For small modulation frequencies, the response $\alpha_{E}(f)$ is flat. With increasing $f \gtrsim 2 \mathrm{GHz}$, it then starts to quickly decrease. The plateau at low frequencies lies at $\alpha_{E}=1.42$, which corresponds exactly to the value of $\alpha_{\text {inj }}$ and $\alpha_{\mathrm{fb}}$ which we had evaluated in the optical injection and feedback setups. There, we also characterized the adiabatic response of the quantum-dot laser to a perturbation of the electric field, such that indeed $\alpha_{\mathrm{inj}}=\alpha_{\mathrm{fb}}=\alpha_{E}(f=0)$ holds.

The decrease at higher modulation frequencies can again be understood by the charge-carrier scattering processes. Here, however, the transfer of the modulation of the ground-state occupation to the off-resonant states becomes less effective for 

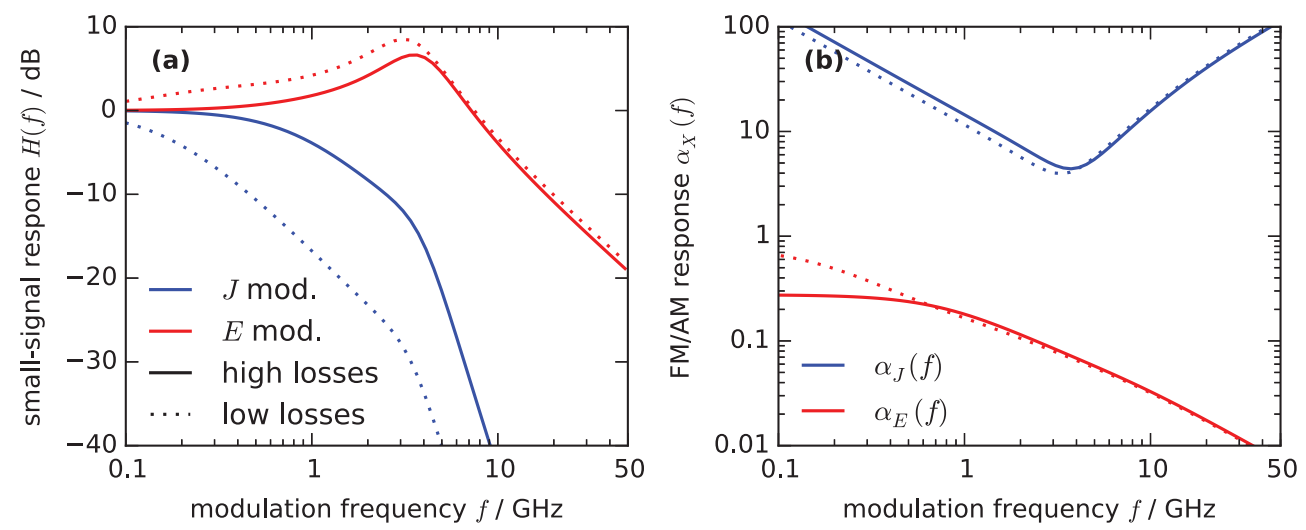

Figure 3.49.: Small-signal modulation response of the quantum-dot laser under current and electric field modulation for scattering rates reduced by a factor 50, cf. Fig. 3.48. The solid curves show the response of the laser with reference reservoir losses $\left(B^{S}=\right.$ $\left.540 \mathrm{~nm}^{2} \mathrm{~ns}^{-1}\right)$, the dotted curves those with lower losses $\left(B^{S}=54 \mathrm{~nm}^{2} \mathrm{~ns}^{-1}\right)$. Modified from [LIN14].

higher frequencies, such that the refractive-index variation becomes smaller. This results in the decrease of $\alpha_{E}$ that we can observe in Fig. $3.48 \mathrm{~b}$. With this we can also understand the different dynamics predicted by our full modeling approach and when using a constant $\alpha$-factor to describe the amplitude-phase coupling. In Fig. 3.32 we had seen that the amplitude-phase coupling is reduced when the laser operates on oscillatory solutions. This is reproduced in the FM/AM response, where for oscillatory solutions, i.e., $f>0, \alpha_{E}$ is indeed smaller than in the limit $f \rightarrow 0$.

Another important result that we can extract from the FM/AM response is the sensitivity of the amplitude-phase coupling to the explicit measurement used for its evaluation. There is experimental evidence that in quantum-dot lasers different measurement techniques will yield different values for the linewidth-enhancement factor [MAR05c], whereas in quantum-well lasers many different techniques will yield comparable results [FOR07].

\subsubsection{Influence of Scattering Rates and Reservoir Losses}

We now take another look on the frequency-response of the quantum-dot laser device in dependence on the scattering rates and charge-carrier reservoir losses. In Sec. 3.7.3 we had seen that the dynamics of the optically injected quantum-dot laser depends crucially on these charge-carrier lifetimes. We will therefore simulate the 
response of the quantum-dot laser to pump-current and electric-field modulation with reduced scattering rates. The resulting response curves are shown in Fig. 3.49.

The decrease of the scattering rates moves the quantum-dot laser into the synchronized-reservoir regime, slightly below the overdamped regime. As we had seen earlier, the modulation bandwidth suffers in this regime, as evident from the strong decay of the small-signal modulation transfer function in Fig. 3.49. The frequency chirp under pump current modulation is greatly enhanced, reaching a minimal value of $\min \left(\alpha_{J}\right)=4.0$, and a subsequent strong increase for $f \gtrsim 3 \mathrm{GHz}$.

The response to the electric field modulation stays comparable to the earlier case (Fig. 3.48). However, the value of $\alpha_{E}$ at low modulation frequency is reduced to 0.29. The reduction of $\alpha_{E}$ is due to the reduced modulation of the off-resonant states when we modulate the resonant charge-carriers directly via the electric field. Since a low value of the amplitude-phase coupling leads to a reduction of the dynamical complexity under optical perturbations [GLO12, PAU12], we can thus expect a slow scattering rate to increase the resistance to unwanted instabilities in quantum-dot lasers.

When additionally decreasing the reservoir losses, the unique quantum-dot features in the modulation response curve become even more pronounced. This is shown by the dotted lines in Fig. 3.49. The resulting high carrier lifetime in the reservoir worsens the modulation response curve for pump current modulation. The frequency response towards optical perturbations is also increased, with $\alpha_{E}(f=0)=0.77$. The chosen very low scattering rates and reservoir losses are extreme values that will probably not be found in realistic devices. So far, however, we can predict that a reduction of the carrier-losses, while improving the device efficiency, can be detrimental to the dynamic device performance.

To obtain a complete picture of the dependence of the frequency response on the scattering rates, we now perform simulations for varying values of the effective scattering rate $S^{\text {eff }}$. We thus evaluate $\alpha_{E}(f=0)$, which we have seen to represent the adiabatic laser response under optical perturbations very well. We also calculate $\alpha_{\mathrm{FM} / \mathrm{AM}}=\min \left(\alpha_{J}\right)$, which should approximate the frequency response under pump current modulation in an interval of relevant modulation frequencies.

The resulting FM/AM response curves are shown in Fig. 3.50. When decreasing the scattering rates from the microscopically calculated values (denoted by the vertical dashed line), the separation between the frequency response towards pump current and optical modulation becomes larger, as we have observed in Fig. 3.49. The value of $\alpha_{E}$ in the constant-reservoir regime is thus reduced to values well below 1 , while the frequency response under pump-current modulation shows a steady increase towards lower scattering rates. The figure shows that only in the limit of instantaneous scattering, $S^{\text {eff }} \rightarrow \infty$, the two measures converge. The frequency 


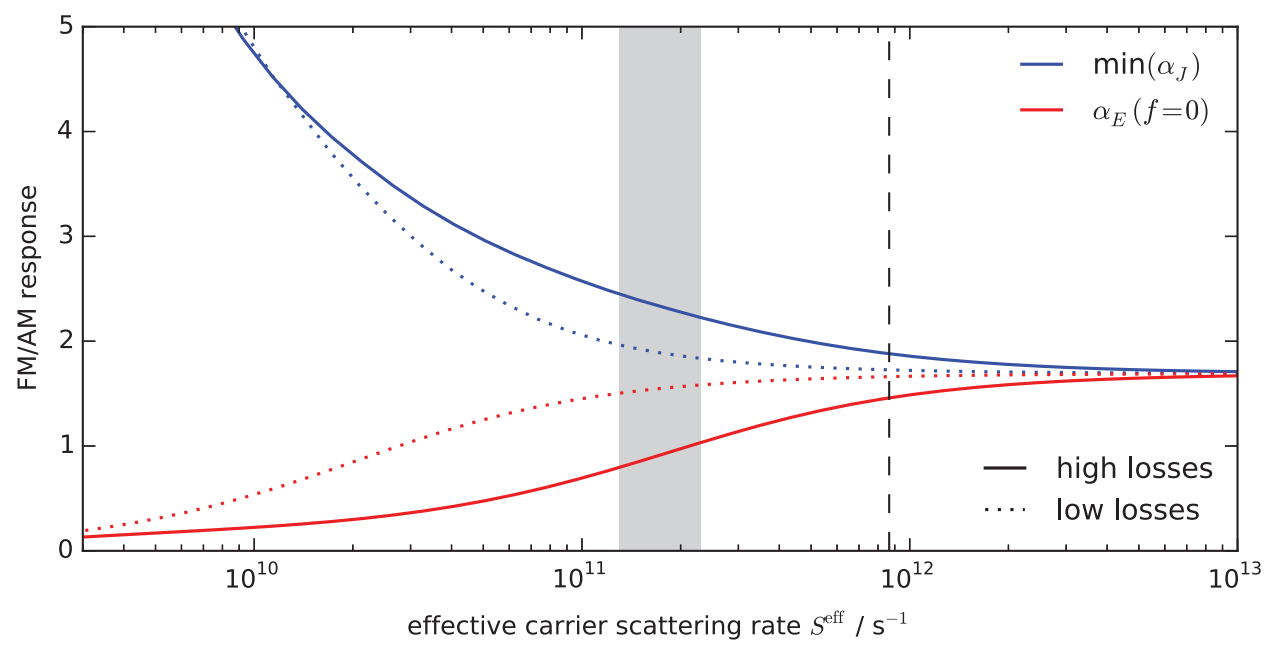

Figure 3.50.: Dependence of the frequency-modulation response on the effective scattering rate $S^{\text {eff }}$. Shown are the minimum of $\alpha_{J}(f)$ under pump current modulation (blue) and $\alpha_{E}(f=0)$ for a static variation of the electric field (red). The resulting values are shown for reference reservoir losses $\left(B^{S}=540 \mathrm{~nm}^{2} \mathrm{~ns}^{-1}\right.$, solid lines) as well as for reduced losses $\left(B^{S}=54 \mathrm{~nm}^{2} \mathrm{~ns}^{-1}\right.$, dotted lines). The pump current was set to twice the respective threshold current at each data point. The shaded gray area denotes the overdamped laser regime, the microscopic rates are denoted by the vertical dashed line. After [LIN14].

response towards different types of perturbation can therefore be expected to differ in quantum-dot lasers.

A reduction of the reservoir losses can be seen to improve the agreement between $\alpha_{E}$ and $\alpha_{J}$. As discussed before, this counteracts the decrease of the amplitudephase coupling under optical perturbations for low scattering rates, and this might increase the sensitivity towards optical instabilities of the quantum-dot laser. On the other hand, for directly modulated quantum-dot lasers the reduction in $\alpha_{J}$ would be beneficial. 


\subsection{Conclusion}

In this chapter we have investigated the dynamics of quantum-dot lasers under external perturbations and highlighted the unique features brought about by the charge-carrier scattering dynamics. We have discussed the relaxation oscillations in quantum-dot lasers as an indicator of the laser stability and its internal time scales. Depending on the effective charge-carrier scattering rate, we have identified three dynamic regimes of laser operation:

(i) For slow charge-carrier scattering, the reservoir states provide a nearly constant carrier-influx into the quantum-dots, leading to pronounced relaxation oscillations. We thus call this regime the "constant-reservoir regime".

(ii) For intermediate scattering rates in the order of the relaxation oscillation angular frequency, the relaxation oscillation damping is strongly increased, leading to overdamped oscillations. This regime is thus called the "overdamped regime".

(iii) For fast scattering rates, the coupling between quantum-dot and reservoir states is strong enough to influence the reservoir carrier density. In this "synchronized regime", the reservoir carriers thus contribute to the light-matter interaction, leading to the reappearance of pronounced relaxation oscillations.

We have subsequently proposed a minimal three-variable rate-equation model in order to explain this behavior. We have found the detailed balance of in and outscattering rates to be a necessary condition for describing the transition between the three dynamic regimes as described above. Analytic expressions for the relaxation oscillation damping and frequency have been derived in the limit of very slow and very fast scattering rates, i.e., for the "constant-reservoir" and "synchronized" regimes.

The small-signal modulation response of the quantum-dot laser has been analyzed in dependence on the scattering rates. A general trend towards higher modulation bandwidths with increasing scattering rates has been found, albeit with a nonlinear dependence of the bandwidth on the scattering rate. The conventional analytic formula used to calculate the intrinsic modulation bandwidth of the laser by evaluating its relaxation oscillation parameters was found to yield inaccurate results in quantum-dot lasers. It greatly exaggerates the expected bandwidth in the constant-reservoir regime while slightly underestimating the bandwidth in the synchronized regime.

We investigated the amplitude-phase coupling in quantum-dot lasers. The complex charge-carrier dynamics lead to a response of the carrier-induced refractiveindex shift that goes beyond the traditional description by an $\alpha$-factor. We have thus simulated the quantum-dot laser dynamics under optical injection and time- 
delayed optical feedback, and compared the results with the predicted dynamics when using an $\alpha$-factor to describe the amplitude-phase coupling. In both cases we have found that the bifurcations of fixed-point solutions are well described by the $\alpha$-factor. Bifurcations of periodic and dynamic solutions, on the other hand, show appreciable differences between the two approaches. The $\alpha$-factor approach was found to be unable to correctly describe the dynamics of the quantum-dot laser in this scenarios, due to the desynchronized dynamics of the real and imaginary part of the optical susceptibility.

Subsequently, we have simplified our full quantum-dot laser model to a much less complex 10-variable rate equation system, that still retains all important features of the quantum-dot laser dynamics. We have shown this model to be suitable for implementation in numeric path-continuation programs, thus opening up the possibility to study the detailed bifurcation structure of quantum-dot lasers in various applications. We have applied the numeric path-continuation to the optical injection setup, again revealing differences in the full description of the carrierinduced susceptibility and the simple $\alpha$-factor approach. Especially in the constantreservoir regime, i.e., for slow scattering, as well as for low non-radiative carrier losses, the differences become pronounced, and here the $\alpha$-factor clearly cannot be used.

In order to explain these observed differences we have investigated the frequency response of the quantum-dot laser subject to modulations of the pump current and the electric field. We have thus simulated FM/AM measurements to determine the frequency-dependence of the amplitude-phase coupling in quantum-dot lasers. Under pump current modulation a high value of the amplitude-phase coupling was found for low modulation frequencies with a plateau at higher frequency, comparable to results for conventional lasers. Unique to quantum-dot lasers is an additional increase for even higher modulation frequencies. We have found this increase to be related to the charge-carrier scattering, making it more pronounced with slower scattering. Under modulation of the electric field, a very different frequency response was found, with a plateau for low modulation frequencies, and a subsequent steep decrease. This phenomenon explains the observed differences in the laser response to optical injection and feedback. Due to the finite scattering time, the amplitude-phase coupling is much lower for a fast modulation of the electric field. The difference between the frequency responses to either pump current or optical modulations was found to be even more pronounced for slower scattering. This explains experimental results which found different values of the amplitude-phase coupling, i.e., the $\alpha$-factor, for different measurement techniques.

Our results thus show that in order to correctly model and describe the quantumdot laser dynamics, an accurate description of the complex charge-carrier dynamics 
is required. We have developed three quantum-dot laser models on different levels of sophistication which fulfill this requirement. 


\section{QuANTUm-Dot Optical Amplifiers}

\subsection{Introduction}

Semiconductor optical amplifiers (SOAs) are optoelectronic devices commonly used in optical data communication networks and signal processing. Semiconductor amplifiers are structurally similar to laser devices, with the difference lying in the absence of an optical cavity. To this end, the waveguide facets of optical amplifiers are commonly treated with an anti-reflection coating in order to increase the optical transmission through the facets and minimize losses. The optical amplifier devices we consider in this work are therefore single-pass devices, where the optical signal coupled into one side of the waveguide structure is ideally passing exactly once across the device and is out-coupled without losses at the back facet. This, however, means that optical amplifiers generally have to be longer than laser devices, in order to provide a long enough interaction time between optical signal and the active medium.

Optical amplifiers are used in optical networks in order to raise the signal power level, e.g., to compensate the inevitable optical losses in long glass-fiber connections. Here, a linear amplification with a low noise figure is required, to ensure low distortion of the input signal [AKI03, BER04, USK05]. On the other hand, nonlinear optical applications, such as four-wave-mixing [AKI02, QAS04, MAJ11a, SCH12e] and cross-gain modulation [KIM09a, MEU10, CON10] for wavelength conversion, as well as regenerative amplification, require a nonlinear response of the optical amplifier. In the nonlinear amplification regime the amplifier response is input-power dependent and will thus lead to signal distortions, which can be utilized, e.g., for pulse shaping [ICS69, SCH88j, AGH09].

Quantum-dot semiconductor optical amplifiers (QDSOAs) show great potential for the use in these applications. They have generally a higher gain bandwidth than conventional devices based on semiconductor quantum-wells due to the inhomogeneous broadening of the localized quantum-dot states, allowing for a broadband amplification. Additionally, due to the coupling to a charge-carrier reservoir by charge-carrier scattering rates in the picosecond range, ultrafast gain recovery 
[BOR00, POE05, LAE06, DOM07, MAJ10, MAJ11] and nonlinear signal processing is possible [CON10, MEU11, SCH12e].

Furthermore, the comparably slow dephasing time of the microscopic inter-band polarization in the localized quantum-dot states [BOR01a, BOR02, KOP11] allows the possibility to directly observe quantum-mechanical effects, such as Rabi-oscillations [STI01b, KAM02, BOR02a, KOL13, CAP14] or self-induced transparency [ICS69, SCH03g]. This could potentially open up new applications in the signal processing of ultra-short, ultra-strong optical pulses.

In this chapter, we will at first derive a delay-differential equation model for the description of the electric field propagation through a quantum-dot semiconductor optical amplifier device, presented in Section 4.2.1. The charge-carrier dynamics will be described in the framework of microscopically calculated scattering rates that we have already successfully employed in the previous chapter. We will then derive a description of the amplified spontaneous emission in the amplifier, and proceed by comparing our theoretical results with experimental measurements of pump-current dependent amplified-spontaneous-emission spectra.

Then, in Section 4.3, we will investigate the possibility of large-signal amplification of optical data signals on optical frequencies corresponding to either the quantum-dot ground-state or excited-state. Subsequently, we will look at the amplifier performance under simultaneous amplification of two optical signals in Section 4.4 .

Lastly, Section 4.5 presents simulation results on coherent pulse-shaping induced by the amplification of ultra-short optical pulses. We will compare the results with experimental measurements, demonstrating the possibility to induce and measure coherent effects in quantum-dot semiconductor optical amplifiers even at room temperature.

In the last Section 4.6, a conclusion of the results presented in this chapter will be given. 


\subsection{Quantum-Dot Semiconductor Optical Amplifier Model}

A model of the quantum-dot semiconductor amplifier must accurately describe the light-matter interaction during the propagation of the optical signal along the device. In contrast to the quantum-dot laser model we had derived in the previous chapter, we can not work with a spatially averaged electric field due to the absence of standing waves. Instead, we must explicitly take the electric field propagation into account, and thus require a spatially resolved description of our dynamical variables.

In the past, models without spatial resolution have been employed for the description of semiconductor amplifiers [BER03b, KUN08, ERN09a, KIM09a]. As soon as strong spatial inhomogeneities arise, such models are, however, bound to fail. We will therefore in the following derive amplifier model equations which include the electric field propagation along the waveguide axis.

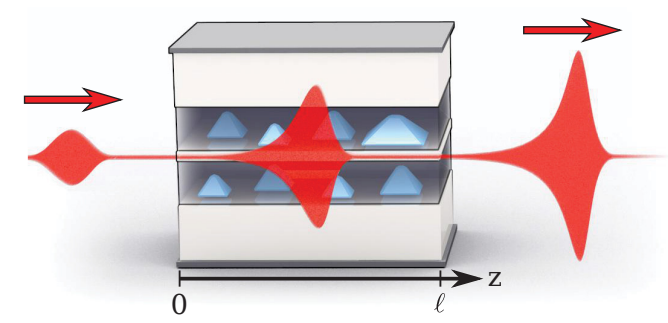

\subsubsection{Electric Field Propagation}

We will now derive the equations governing the propagation of the electric field along the amplifier device. We denote the propagation axis as $z$, as sketched in Fig. 4.1. The real electric field amplitude is then written as

$$
\mathcal{E}(z, t)=\frac{1}{2}\left[E^{+}(z, t) e^{i k z}+E^{-}(z, t) e^{-i k z}\right] e^{-i \omega t}+\text { c.c. },
$$

where we have introduced the slowly varying field amplitudes $E^{ \pm}$, describing the forward $(+)$ and backward $(-)$ propagating electric field. The wave number is given by $k$, and $\omega$ is the optical frequency of the reference frame. From Maxwell's equations we can derive the propagation equations for the electric field amplitudes within the slowly varying envelope approximation:

$$
\left(\partial_{t} \pm v_{g} \partial_{z}\right) E_{ \pm}(z, t)=\frac{i \omega \Gamma}{2 \varepsilon_{\mathrm{bg}} \varepsilon_{0}} P_{ \pm}(z, t)=: S_{ \pm}(z, t),
$$


with the group velocity $v_{g} \equiv \frac{c_{0}}{n_{\mathrm{bg}}}$ and the macroscopic slowly varying polarization amplitude $P_{ \pm}(z, t)$. We summarize the right hand side in a general source term $S_{ \pm}(z, t)$.

The numerical solution of the above partial differential equation requires a spatial discretization into very fine sections of length $h v_{g}$, where $h$ is the numerical timestep, in order to ensure numerical stability [PRE07]. For a device length in the order of $\mathrm{mm}$, this results in a high number of spatial discretization points and thus presents an inefficient way of modeling the electric field propagation [RAD06, ROS11c]. A more elegant approach is the formulation of the problem as a delay-differential equation system [ROS11d, JAV12], which we will do in the following.

\section{Delay-Differential-Equation Model}

The partial differential equation Eq. (4.2) can be transformed into a co-moving frame, by defining a new time variable

$$
t^{\prime}:=t \pm \frac{z}{v_{g}} .
$$

By expanding the total derivative $\frac{\mathrm{d}}{\mathrm{d} z}$, we can thus write

$$
\frac{\mathrm{d}}{\mathrm{d} z} E_{ \pm}\left(z, t^{\prime}\right)=\left[ \pm \frac{1}{v_{g}} \frac{\partial}{\partial t^{\prime}}+\frac{\partial}{\partial z}\right] E_{ \pm}\left(z, t^{\prime}\right)= \pm \frac{1}{v_{g}} S_{ \pm}\left(z, t^{\prime}\right) .
$$

Now we describe the optical amplifier by a number of $Z$ sections along the propagation axis, such that the distance between two discretization points is given by $\Delta z:=\ell / Z$. Integrating Eq. (4.4) over the length of $\Delta z$ thus yields:

$$
\begin{aligned}
E_{ \pm}(z, t) & =E_{ \pm}(z \mp \Delta z, t-\Delta t)+\frac{1}{v_{g}} \int_{0}^{\Delta z} S_{ \pm}\left(z \mp z^{\prime}, t-\frac{z^{\prime}}{v_{g}}\right) \mathrm{d} z^{\prime} \\
& \approx E_{ \pm}(z \mp \Delta z, t-\Delta t)+\frac{\Delta t}{2}\left[S_{ \pm}(z, t)+S_{ \pm}(z \mp \Delta z, t-\Delta t)\right]
\end{aligned}
$$

Here we have introduced $\Delta t:=\frac{\Delta z}{v_{g}}$. The integral over the source term was approximated by its values at the end points of the integration interval. This approximation is valid for negligible change of $S_{ \pm}$along the integration path, i.e., for a sufficiently small space discretization step. The electric field at time $t$ now depends on the values of $E_{ \pm}, S_{ \pm}$at time $t-\Delta t$, which introduces a time-delay into the equations.

The electric field in each of the spatial sections along the amplifier device thus couples to the time-delayed electric field in the neighboring sections, with the time $\Delta t$ describing the time needed for the electric field propagation along the length 


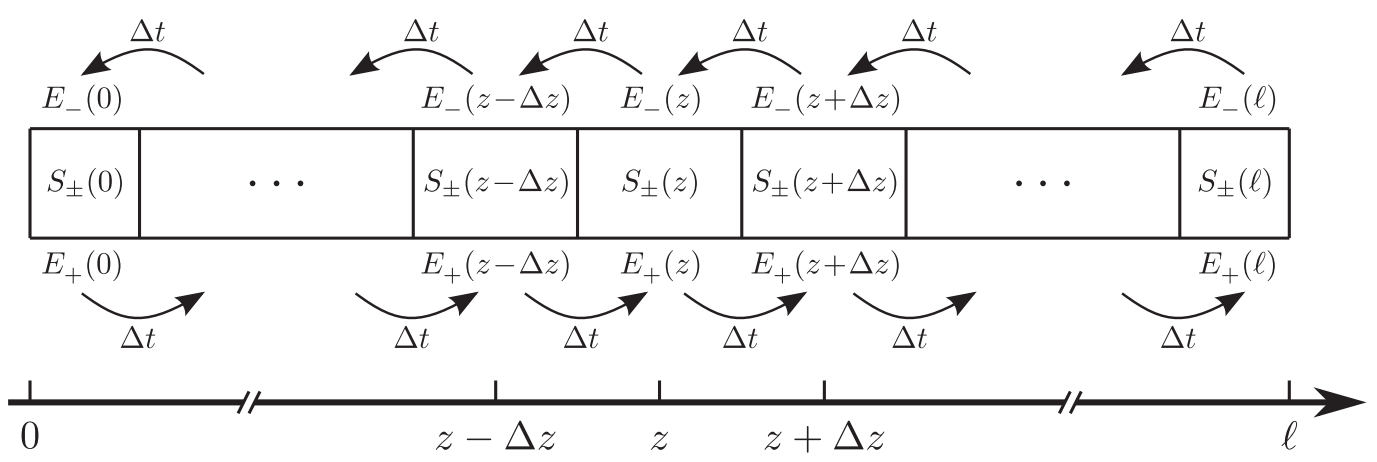

Figure 4.2.: Space discretization scheme of the QDSOA device with length $\ell$ used in the DDE model. The forward and backward propagating electric field, $E_{ \pm}$, is described at points $n \cdot \Delta z, n \in\{0, \ldots, Z\}$ with $\Delta z=\ell / Z$. The corresponding material quantities and source terms, $S_{ \pm}$, are treated as constant within each of the $Z$ sections given by the intervals $z \in\left[n \Delta z-\frac{\Delta z}{2}, n \Delta z+\frac{\Delta z}{2}\right]$, which are centered around the discretization points of the electric field. Cf. [SCH88i, SCH88j, SCH90b, JAV12].

of one section [ROS11d, JAV12]. The resulting discretization scheme is illustrated in Fig. 4.2.

\subsubsection{Quantum-Dot Material Equations}

The material dynamics within each amplifier section are described within the Maxwell-Bloch approach, as derived in Sec. 2.3. We extend these equations by taking into account the microscopic polarization $p_{m, \pm}^{j}$ corresponding to the forward and backward propagating parts of the electric field. Within the slowly varying envelope approximation, we can take these into account separately by neglecting the fast oscillating terms $\propto e^{ \pm 2 i \omega t}$ and $\propto e^{ \pm 2 i k z}$. The material equations then read

$$
\begin{aligned}
\frac{\mathrm{d}}{\mathrm{d} t} p_{m, \pm}^{j}(z, t)= & -\left[i\left(\omega_{m}^{j}-\omega\right)+\frac{1}{T_{2}}\right] p_{m, \pm}^{j}(z, t) \\
& -i \frac{\mu_{m}}{2 \hbar}\left(\rho_{e, m}^{j}(z, t)+\rho_{h, m}^{j}(z, t)-1\right) E_{ \pm}(z, t) \\
\frac{\mathrm{d}}{\mathrm{d} t} \rho_{b, m}^{j}(z, t)= & -\frac{1}{\hbar} \operatorname{Im}\left[p_{m,+}^{j}(z, t) \mu_{m}^{*} E_{+}^{*}(z, t)\right]-\frac{1}{\hbar} \operatorname{Im}\left[p_{m,-}^{j}(z, t) \mu_{m}^{*} E_{-}^{*}(z, t)\right] \\
& -W_{m} \rho_{e, m}^{j}(z, t) \rho_{h, m}^{j}(z, t)+\left.\frac{\partial}{\partial t} \rho_{b, m}^{j}(z, t)\right|_{\mathrm{sc}}
\end{aligned}
$$


Here, we again denote the localized quantum-dot states by $m \in\{$ GS, ES $\}$ and their subgroup index by $j$. The macroscopic slowly varying polarization amplitude is calculated from the microscopic contributions:

$$
P_{ \pm}(z, t)=\frac{2 N^{\mathrm{QD}}}{h^{\mathrm{QW}}} 2 \sum_{j, m} \nu_{m} f(j) \mu_{m}^{*} p_{m, \pm}^{j}(z, t) .
$$

The reservoir charge-carrier density follows the dynamic equation

$$
\frac{\mathrm{d}}{\mathrm{d} t} w_{b}(z, t)=\frac{J}{e_{0}}-r_{\text {loss }}^{w}(z, t)-2 N^{\mathrm{QD}} \sum_{j, m} \nu_{m} f(j) S_{b, m}^{j, \mathrm{cap}}(z, t),
$$

with the scattering contributions between the reservoir and quantum-dot states given by Eqs. (2.66). The above equations are defined for each space discretization point along the amplifier device, allowing for a spatially inhomogeneous distribution of the charge-carrier distribution, as encountered in long amplifier devices [FAN95, FEH02].

\subsubsection{Modeling of Spontaneous Emission}

In comparably long amplifier devices the radiation spontaneously emitted along the propagation axis will be subject to stimulated amplification. This amplified stimulated emission (ASE) is important for the device characteristics and performance, as it can become strong enough to influence the charge-carrier dynamics [BER04a, MEL08, BAV10]. Furthermore, it will create an - in most cases unwanted noise background that will deteriorate the signal quality of the optical input signal.

The correct description of the amplified spontaneous emission is therefore important. In general there exist two appropriate modeling approaches: the deterministic description of the ASE power spectral density in frequency space [MEU08a, KIM09a] and the stochastic description in time-domain [MEL08, ROS11c]. Here, we will employ the stochastic description, which simplifies the inclusion of time-varying input signals.

We therefore phenomenologically add an additional source term on the righthand side of Eq. (4.4), modeling the stochastic spontaneous emission added to the propagating electric field:

$$
\frac{\mathrm{d}}{\mathrm{d} z} E_{ \pm}\left(z, t^{\prime}\right)=\frac{1}{v_{g}}\left[S_{ \pm}\left(z, t^{\prime}\right)+S_{ \pm}^{\mathrm{sp}}\left(z, t^{\prime}\right)\right] .
$$


The electric field propagation along one space-discretization section is again determined by integration of Eq. (4.9) over the interval $\Delta z$ :

$$
\begin{aligned}
E_{ \pm}(z, t) \approx & E_{ \pm}(z \mp \Delta z, t-\Delta t)+\frac{\Delta t}{2}\left[S_{ \pm}(z, t)+S_{ \pm}(z \mp \Delta z, t-\Delta t)\right] \\
& +\frac{1}{v_{g}} \int_{0}^{\Delta z} S_{ \pm}^{\mathrm{sp}}\left(z \mp z^{\prime}, t-\frac{z^{\prime}}{v_{g}}\right) \mathrm{d} z^{\prime} .
\end{aligned}
$$

The spontaneous emission source term must account for all optical transitions in the inhomogeneously broadened QD ensemble. Let $\eta_{m}^{j}(z, t)$ describe the spontaneous emission contribution of the $j^{\text {th }}$ subgroup of the $m^{\text {th }}$ localized state. We write for the electric field spontaneously added to the propagating field along $\Delta z$ :

$$
\frac{1}{v_{g}} \int_{0}^{\Delta z} S_{ \pm}^{\mathrm{sp}}\left(z \mp z^{\prime}, t-\frac{z^{\prime}}{v_{g}}\right) \mathrm{d} z^{\prime} \equiv \sum_{m, j} \eta_{m}^{j}(z, t) .
$$

The spontaneous emission of an optical transition has a finite linewidth given by its homogeneous broadening. In order to correctly implement the spectral properties of the amplified spontaneous emission, the spontaneously emitted field $\eta_{m}^{j}(z, t)$ must therefore have the correct lineshape and center frequency. It is thus not possible to describe the spontaneous emission by white noise, which would produce a flat noise spectrum, but must be rather modeled using colored noise. We implement this colored noise by two-dimensional Ornstein-Uhlenbeck processes. The time evolution of each of the respective noise signals is thus given by the following stochastic differential equation:

$$
\frac{\mathrm{d}}{\mathrm{d} t} \eta_{m}^{j}(z, t)=-\gamma \eta_{m}^{j}(z, t)+\sqrt{D_{\mathrm{sp}, m}^{j}(z, t)} \tilde{\xi}_{m}^{j}(z, t),
$$

where $\tilde{\xi}(z, t)$ is a complex Gaussian white noise process, which is $\delta$-correlated both in $z$ and $t$. The correlation time of $\eta_{m}^{j}$ is given by $\gamma^{-1}$. The noise signal then fulfills the following properties [GAR85]:

$$
\begin{aligned}
\left\langle\operatorname{Re} \eta_{m}^{j}(z, t)\right\rangle=\left\langle\operatorname{Im} \eta_{m}^{j}(z, t)\right\rangle & =0 \\
\left\langle\left|\eta_{m}^{j}(z, t)\right|^{2}\right\rangle & =\frac{D_{\mathrm{sp}, m}^{j}(z, t)}{\gamma} \\
\left\langle\eta_{m}^{j}(z, t) \eta_{m}^{j}{ }^{*}\left(z^{\prime}, t+\tau\right)\right\rangle & \approx \frac{D_{\mathrm{sp}, m}^{j}(z, t)}{\gamma} e^{-\gamma|\tau|} \delta_{z, z^{\prime}} .
\end{aligned}
$$

Eq. (4.15) is valid only under the assumption of a slowly varying noise amplitude $\partial_{t} D_{\mathrm{sp}}(z, t) \ll \gamma$, such that within one correlation time $\gamma^{-1}$ the spontaneous emis- 
sion amplitude can be assumed as constant. Using the Wiener-Khinchin-theorem, this relation can be used to calculate the power spectrum $\mathcal{S}_{\eta_{m}^{j}}(z, \omega)$ of $\eta_{m}^{j}(z, t)$ :

$$
\begin{aligned}
\mathcal{S}_{\eta_{m}^{j}}(z, \omega) & =\frac{1}{2 \pi} \int_{-\infty}^{\infty}\left\langle\eta_{m}^{j}(z, t) \eta_{m}^{j}{ }^{*}(z, t+\tau)\right\rangle e^{-i \omega \tau} \mathrm{d} \tau \\
& =\frac{D_{\mathrm{sp}, m}^{j}(z)}{\pi} \frac{1}{\omega^{2}+\gamma^{2}},
\end{aligned}
$$

which yields a Lorentzian line shape with a width (FWHM) of $2 \gamma$. We thus identify $\gamma=\left(T_{2}\right)^{-1}$, such that the noise linewidth equals the homogeneous linewidth of the QD transitions.

Using the noise correlation properties, the average power that is added to the electric field by the noise can be calculated. Combining Eqs. (4.10) and (4.11) and summarizing the deterministic source terms in a combined variable, $\widetilde{S}_{ \pm}^{\text {stim }}$, yields for the electric field:

$$
\begin{aligned}
E_{ \pm}(z, t) & =E_{ \pm}(z \mp \Delta z, t-\Delta t)+\widetilde{S}_{ \pm}^{\mathrm{stim}}(z, t)+\sum_{m, j} \eta_{m}^{j}(z, t) \\
\left\langle\left|E_{ \pm}(z, t)\right|^{2}\right\rangle & =\left|E_{ \pm}(z \mp \Delta z, t-\Delta t)+\widetilde{S}_{ \pm}^{\mathrm{stim}}(z, t)\right|^{2}+\sum_{m, j}\left\langle\left|\eta_{m}^{j}(z, t)\right|^{2}\right\rangle \\
& =\left|E_{ \pm}(z \mp \Delta z, t-\Delta t)+\widetilde{S}_{ \pm}^{\mathrm{stim}}(z, t)\right|^{2}+\sum_{m, j} T_{2} D_{\mathrm{sp}, m}^{j}(z, t)
\end{aligned}
$$

where we have used the zero mean property, $\left\langle\eta_{m}^{j}\right\rangle=0$. On average, the spontaneous emission thus increases the squared modulus of the electric field along one space discretization step during the propagation time $\Delta t$ by $\sum_{m, j} T_{2} D_{\mathrm{sp}, m}^{j}(z, t)$. Or, written in terms of a time derivative:

$$
\left.\frac{\partial}{\partial t}\left|E_{ \pm}(z, t)\right|^{2}\right|_{\mathrm{sp}}=\frac{T_{2}}{\Delta t} \sum_{m, j} D_{\mathrm{sp}, m}^{j}(z, t) .
$$

In the photon picture, the average change of the electric field energy density due to the spontaneous emission can be calculated:

$$
\begin{aligned}
\left.\frac{\partial}{\partial t} u(z, t)\right|_{\mathrm{sp}} & =\left.\frac{\varepsilon_{\mathrm{bg}} \varepsilon_{0}}{2} \frac{\partial}{\partial t}\left|E_{ \pm}(z, t)\right|^{2}\right|_{\mathrm{sp}} \\
& =\beta \frac{2 N^{\mathrm{QD}} \Gamma}{h^{\mathrm{QW}}} \sum_{m, j} \nu_{m} f(j) \hbar \omega_{m}^{j} W_{m} \varrho_{\mathrm{e}, m}^{j}(z, t) \varrho_{\mathrm{h}, m}^{j}(z, t),
\end{aligned}
$$


with the notation as introduced in Sec. 2.4. The individual noise strengths are thus given by

$$
D_{\mathrm{sp}, m}^{j}(z, t)=\frac{\Delta t}{T_{2}} \frac{2 \beta \Gamma \hbar \omega_{m}^{j} 2 N^{\mathrm{QD}}}{\varepsilon_{\mathrm{bg}} \varepsilon_{0} h^{\mathrm{QW}}} \nu_{m} f(j) \frac{1}{2}\left[R_{\mathrm{sp}, m}^{j}(z, t)+R_{\mathrm{sp}, m}^{j}(z \mp \Delta z, t-\Delta t)\right],
$$

where the average of the spontaneous emission rate at the endpoints of the integration interval $[z, z \mp \Delta z]$ was taken, defined by

$$
R_{\mathrm{sp}, m}^{j}(z, t):=W_{m} \varrho_{\mathrm{e}, m}^{j}(z, t) \varrho_{\mathrm{h}, m}^{j}(z, t) .
$$

The spontaneous emission noise thus depends on the optical frequency and on the occupation of the individual quantum-dot subgroups. 


\subsection{Large-Signal Amplification in Quantum-Dot Amplifiers}

The device performance of semiconductor optical amplifiers is generally limited by two competing effects. On the one hand, the maximum achievable optical output power is limited by the charge-carriers available for stimulated emission. The gain of the amplifier will therefore decrease when the optical power becomes too large. This effect is known as gain saturation.

On the other hand, a too small optical signal will significantly reduce the signalto-noise ratio, as the spontaneous emission background will dominate the output. Noise effects thus play an important role in the amplification of optical data signals [BON11, WIL12c]. A strong noise background will negatively impact the signal quality by distorting the corresponding optical output, and potentially corrupting the transmitted data stream.

In this section we will investigate the amplifier performance in terms of gain and signal quality under large-signal amplification conditions. In order to accurately model the spontaneous emission noise we will perform pump-current dependent simulations of the amplified spontaneous emission spectra and compare them with experimental results ${ }^{1}$. Then, we calculate the amplifier performance for different large-signal input powers, with data signals centered on either the ground-state or excited-state energies.

\subsubsection{Calculation of Amplified Spontaneous Emission Spectra}

We will employ the previously derived delay-differential equation model to simulate the amplified spontaneous emission of real quantum-dot amplifier devices. The modeled device is a $3 \mathrm{~mm}$ long dot-in-a-well structure, consisting of ten $5 \mathrm{~nm}$ thick InGaAs quantum-wells, each embedding a density of $3 \times 10^{10} \mathrm{~cm}^{-2}$ InAs quantumdots, with a shallow-etched, $4 \mu \mathrm{m}$ wide ridge waveguide.

The pump-current dependent optical power spectra of the amplified spontaneous emission have been measured, as shown in Fig. $4.3 \mathrm{~b}$. The experimental results reveal a strong peak around $\lambda=1300 \mathrm{~nm}$, corresponding to the quantum-dot groundstate emission. With increasing pump current $j$, the ground-state peak increases until it saturates around $j=300 \mathrm{~mA}$, after which it decreases again. Around $\lambda=1200 \mathrm{~nm}$ a second peak arises with increasing pump current, corresponding to the quantum-dot excited state. This peak saturates around $j=800 \mathrm{~mA}$, with a slight decrease at higher pump currents. the whole spectrum exhibits a red-shift towards higher wavelengths with increasing pump current.

\footnotetext{
Parts of this section have been published in [WIL12c, LIN14a].

${ }^{1}$ The experimental data was kindly provided by Holger Schmeckebier, Inst. f. Festkörperphysik, TU Berlin.
} 

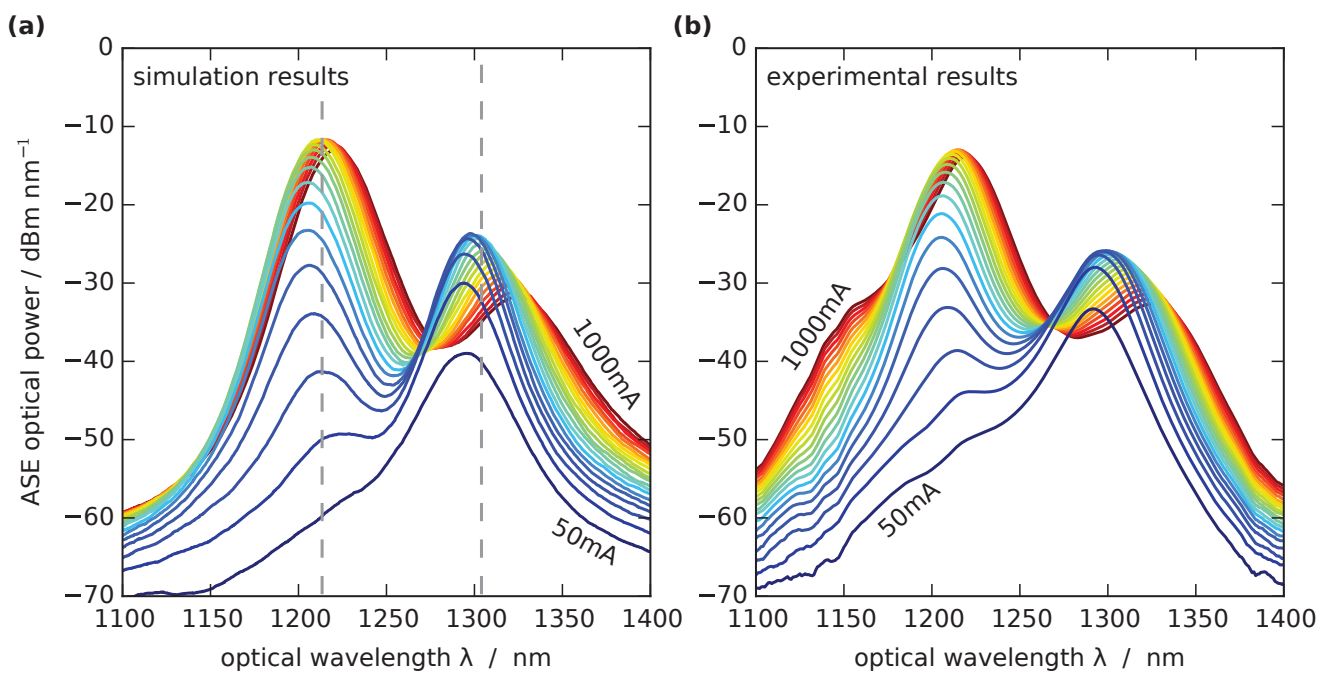

Figure 4.3.: Comparison the simulated and measured ASE spectra. (a) shows the simulated ASE spectra for currents between $50 \mathrm{~mA}$ (dark blue) and $1000 \mathrm{~mA}$ (dark red), increased in steps of $50 \mathrm{~mA}$. The vertical dashed gray lines denote the center wavelengths of the considered optical data signals at $\lambda=1305 \mathrm{~nm}(\mathrm{GS})$ and $\lambda=1215 \mathrm{~nm}$ (ES). (b) Corresponding experimentally measured spectra, kindly provided by Holger Schmeckebier, TU Berlin.

We proceed by fitting the experimental spectral data with the results from our simulations. The resulting model parameters are given in Table 4.1. Here, we have treated the quantum-dot density $N^{\mathrm{QD}}$, the reservoir loss rates $A^{S}, B^{S}$, the quantum-dot dipole moments $\mu_{m}$, their inhomogeneous broadening $\Delta E_{\text {inh }}^{m}$, and their dephasing times $T_{2}^{m}$, as well as the geometric confinement factor $\Gamma$ and the spontaneous emission ratio $\beta$ as fitting parameters. In order to reproduce the measured spectra, we must include a pump-current efficiency, $\eta=0.4$, describing the ratio of injected charge-carriers that reach the optically active region. This reduced efficiency models the losses during carrier transport from the contacts and in the separate confinement heterostructure that are not explicitly included in the model. The relation between the experimental pump current $j$ and the effective pump current density that enters in our model is given by

$$
J=\frac{\eta}{a_{L} A_{\mathrm{act}}} j
$$

where $A_{\text {act }}=3 \mathrm{~mm} \times 4 \mu \mathrm{m}$ is the waveguide area. 
Table 4.1.: Model parameters used in sections 4.3 and 4.4, unless stated otherwise.

\begin{tabular}{lll}
\hline \hline SYMBOL & VALUE & MEANING \\
\hline$N^{\mathrm{QD}}$ & $3 \times 10^{10} \mathrm{~cm}^{-2}$ & QD density per layer \\
$a_{L}$ & 10 & number of layers \\
$h^{\mathrm{QW}}$ & $5 \mathrm{~nm}$ & QW layer height \\
$n_{\mathrm{bg}}$ & 3.77 & background index \\
$\Delta E_{\mathrm{inh}}^{\mathrm{GS}}$ & $30 \mathrm{meV}$ & QD GS inhomogeneous broadening FWHM \\
$\Delta E_{\mathrm{inh}}^{\mathrm{ES}}$ & $55 \mathrm{meV}^{-1}$ & QD ES inhomogeneous broadening FWHM \\
$A^{S}$ & $0.7 \mathrm{~ns}^{-1}$ & QW linear recombination rate \\
$B^{S}$ & $50 \mathrm{~nm}^{2} \mathrm{~ns}^{-1}$ & QW bimolecular recombination rate \\
$W_{\mathrm{GS}}$ & $0.44 \mathrm{~ns}^{-1}$ & GS spontaneous recombination rate \\
$W_{\mathrm{ES}}$ & $0.55 \mathrm{~ns}^{-1}$ & ES spontaneous recombination rate \\
$\beta$ & $3.5 \times 10^{-4}$ & spontaneous emission ratio \\
$\mu_{\mathrm{GS}}$ & $0.62 \mathrm{~nm}_{0} \times\left(1+\frac{j}{2.5 \mathrm{~A}}\right)^{-\frac{1}{2}}$ & QD GS transition dipole moment \\
$\mu_{\mathrm{ES}}$ & $0.85 \mathrm{~nm}_{0} \times\left(1+\frac{j}{2.5 \mathrm{~A}}\right)^{-\frac{1}{2}}$ & QD ES transition dipole moment \\
$T_{2}^{\mathrm{GS}}$ & $200 \mathrm{fs} \times\left(1+\frac{j \mathrm{~mA}}{300}\right)^{-1}$ & QD GS polarization dephasing time \\
$T_{2}^{\mathrm{ES}}$ & $300 \mathrm{fs} \times\left(1+\frac{j}{300 \mathrm{~mA}}\right)^{-1}$ & QD ES polarization dephasing time \\
$\Gamma$ & 0.045 & Geometric confinement factor \\
$\Delta E_{e}\left(\Delta E_{h}\right)$ & $95 \mathrm{meV}(50 \mathrm{meV})$ & electron (hole) QD GS localization energy \\
$\Delta_{e}\left(\Delta_{h}\right)$ & $60 \mathrm{meV}(25 \mathrm{meV})$ & electron (hole) QD GS-ES energy spacing \\
$\eta$ & 0.4 & pump current efficiency \\
$Z$ & 30 & number of space discretization steps \\
\hline \hline
\end{tabular}

In order to correctly describe the pump-current dependence of the amplified spontaneous emission spectra, we introduce a pump-current dependence of the gain, which is modeled as a dependence of the transition dipole moments,

$$
\left|\mu_{m}(j)\right|^{2}=\frac{\left|\mu_{m}^{0}\right|^{2}}{1+\frac{j}{2.5 \mathrm{~A}}},
$$

with $\mu_{m}^{0}$ the corresponding dipole moments at $j=0$. At the maximum current of $j=1000 \mathrm{~mA}$ that we will use here, the square of the dipole moment, and thus the gain, is reduced to $71 \%$ of its original value. This phenomenological change of the resulting gain is used to explain effects that are not intrinsically included in the quantum-dot amplifier model, e.g., the quantum-confined stark effect at higher bias voltages that leads to a decrease of the dipole moment [SCH99, JIN04]. Furthermore, the dephasing time $T_{2}$ of the quantum-dot transitions is known 


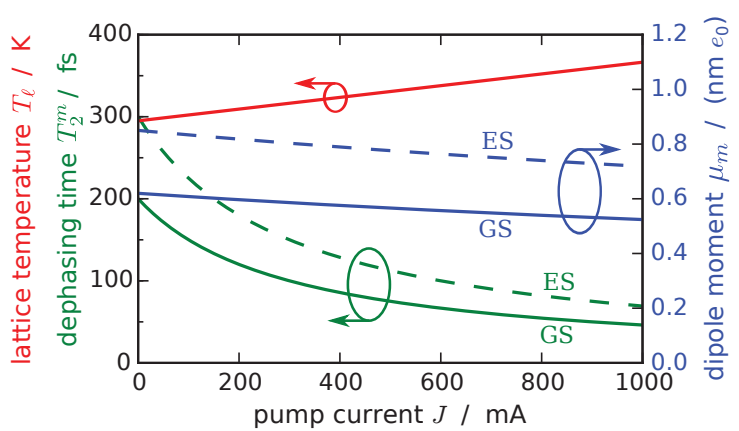

Figure 4.4: Fitted pump-current dependence of the model parameters used in Secs. 4.3 and 4.4 . Shown are the quantum-dot dephasing time (green) and dipole moment (blue), both for ground state (solid) and excited state (dashed), as well as the lattice temperature (red) in dependence of the pump current $j$.

to depend on charge-carrier density and temperature [BOR02, NIL05, LOR06, KOP11, GOL14]. We thus introduce a current-dependent dephasing time,

$$
T_{2}^{m}(j)=\frac{T_{2}^{m, 0}}{1+\frac{j}{300 \mathrm{~mA}}},
$$

where $T_{2}^{m, 0}$ denotes the corresponding dephasing times at $j=0$. We assume a longer dephasing time for the quantum-dot excited state compared to the groundstate [LOR06a].

Across the pump-current range from $50 \mathrm{~mA}$ to $1000 \mathrm{~mA}$ a red-shift of the gainspectrum by about $30 \mathrm{~nm}$ can be observed. We attribute this shift to band-gap renormalization due to many-body interactions [HAU89, CHO99, SCH01d, CHO05, LIN10] as well as a Varshni-shift induced by an increase of the active-medium lattice temperature. Experimental and theoretical works predict a band-gap renormalization in the order of below $10 \mathrm{~nm}$ due to many-body effects [ODR10a, SHA11b]. In order to explain the observed red-shift of $\approx 30 \mathrm{~nm}$, we therefore need to additionally implement a pump-current dependent lattice temperature

$$
T_{\ell}(j)=295 \mathrm{~K}+\frac{1 \mathrm{~K}}{14 \mathrm{~mA}} \times j,
$$

which leads to a red-shift of $0.4 \mathrm{meV} \mathrm{K}^{-1}$ of the band-gap energy [GUE13, FRA13], and reproduces the observed shift in the measured spectra. The resulting pump-current dependent parameter fits are shown in Fig. 4.4.

For the correct description of the reservoir losses we furthermore include linear and bimolecular loss rates, $A^{S}$ and $B^{S}$, in the dynamic equations of the reservoir carriers:

$$
r_{\mathrm{loss}}^{w}=A^{S} \sqrt{w_{e} w_{h}}+B^{S} w_{e} w_{h} .
$$


Figure 4.5: Pump-current dependence of the optical small-signal gain at the ground-state $(\lambda=1305 \mathrm{~nm}$, red) and the excited-state $(\lambda=1215 \mathrm{~nm}$, blue). Shown are the simulated curves (solid lines) together with the experimental data (filled circles), kindly provided by Holger Schmeckebier, TU Berlin.

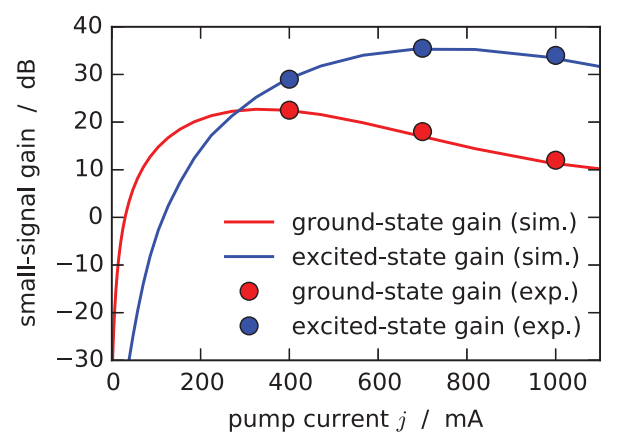

The dependence of the linear loss term on the geometric mean $\sqrt{w_{e} w_{h}}$ is chosen such that the same value of $r_{\text {loss }}^{w}$ can be used in both the electron and hole reservoir equations, ensuring charge conservation. In the earlier discussion of quantum-dot laser dynamics we had neglected the linear loss term, due to the comparably high reservoir carrier densities above the laser threshold, for which the higher-order loss term dominates. Here, on the other hand, we have a large variation in the pump current, such that the linear loss rate $A^{S}$ becomes important to correctly reproduce the carrier-dependent dynamics also for low currents.

Using these model parameters we now simulate the amplified spontaneous emission spectra at the pump currents corresponding to the experimental values. The resulting spectra are shown in Fig. 4.3 a. We can reproduce the pump-current dependence of the amplified spontaneous emission very well both qualitatively and quantitatively. The experimental spectra reveal a shoulder around $\lambda=1150 \mathrm{~nm}$ at higher pump currents. We identify this as the reservoir band edge. Since we do not explicitly take into account the spontaneous emission of the reservoir states, the simulations cannot reproduce this part of the spectrum. In the following, we will however limit ourselves to the amplification of signals centered at the quantumdot ground and excited state energies. We can therefore neglect the discrepancy between the spectra at this wavelength.

Apart from the amplified spontaneous emission, we additionally calculate the small-signal gain for different pump currents, as shown in Fig. 4.5. Here we choose $\lambda=1305 \mathrm{~nm}$ at the ground-state center frequency and $\lambda=1215 \mathrm{~nm}$ for the excited state. The simulated results match the experimentally obtained data excellently. The corresponding gain curves match the qualitative behavior of the amplified spontaneous emission spectra, with a saturation and subsequent decrease of the gain.

We can conclude that our simulations quantitatively reproduce the experimental results very well. In the next section, we will therefore employ the quantum- 
dot-semiconductor-amplifier model with the obtained parameters to simulate the performance under amplification of optical large-signal data streams.

\subsubsection{Gain Saturation}

Large-signal amplification refers to the use of optical signals that can be large enough to influence the charge-carrier occupation along the amplifier device, and thus introduce nonlinearities. The charge-carrier distribution in the quantum-dot amplifier can then be substantially perturbed, leading to an input-power dependent response of the device. In order to quantify this response, we simulate the amplifier under the injection of a constant optical input signal. As we have seen in the previous section, the quantum-dot amplifier is capable of amplifying optical signals on the ground-state and excited-state energies. We will therefore investigate the device performance under amplification of signals at either of the two corresponding wavelengths.

We thus model the electric field at the input facet as

$$
E_{+}(0, t)=A_{\text {in }}^{m} e^{-i \omega^{m} t}
$$

where the input amplitude is related to the optical power by

$$
P_{\text {in }}^{m}=A_{\text {beam }} \frac{n_{\text {bg } \varepsilon_{0} c_{0}}}{2}\left|A_{\text {in }}^{m}\right|^{2},
$$

with the effective transversal mode area $A_{\text {beam }}$. In Eq. (4.29) the frequency detuning $\omega^{m}(m \in\{\mathrm{GS}, \mathrm{ES}\})$ of the input signal relative to the carrier frequency is chosen to yield $\lambda=1305 \mathrm{~nm}$ for signals centered on the ground-state energy, and $\lambda=1215 \mathrm{~nm}$ for the excited-state. We can then evaluate the device gain in the steady-state by

$$
G\left(P_{\text {in }}\right)=\frac{\left|E_{+}(\ell, t)\right|^{2}}{\left|E_{+}(0, t)\right|^{2}} .
$$

The power-dependent gain for both wavelengths is plotted in Fig. 4.6.

Figure 4.6 a shows the ground-state gain in dependence on the optical input power for different pump currents. The small-signal gain, i.e., at low input powers, shows the behavior that we have seen before in Fig. 4.5, with an increase up to a pump current of $j=400 \mathrm{~mA}$ and a subsequent decrease for higher $j$. The gain curves are flat for low input powers (linear regime), but start decreasing once $P_{\text {in }}$ becomes large enough (nonlinear regime). This effect is known as gain saturation [BER04, USK05, MEU08a]. In order to characterize the onset of the nonlinear regime, the saturation input power $P_{\mathrm{in}}^{\text {sat }}$ is defined as the input power at which the gain is reduced to half the small-signal gain. Depending on the application, different 


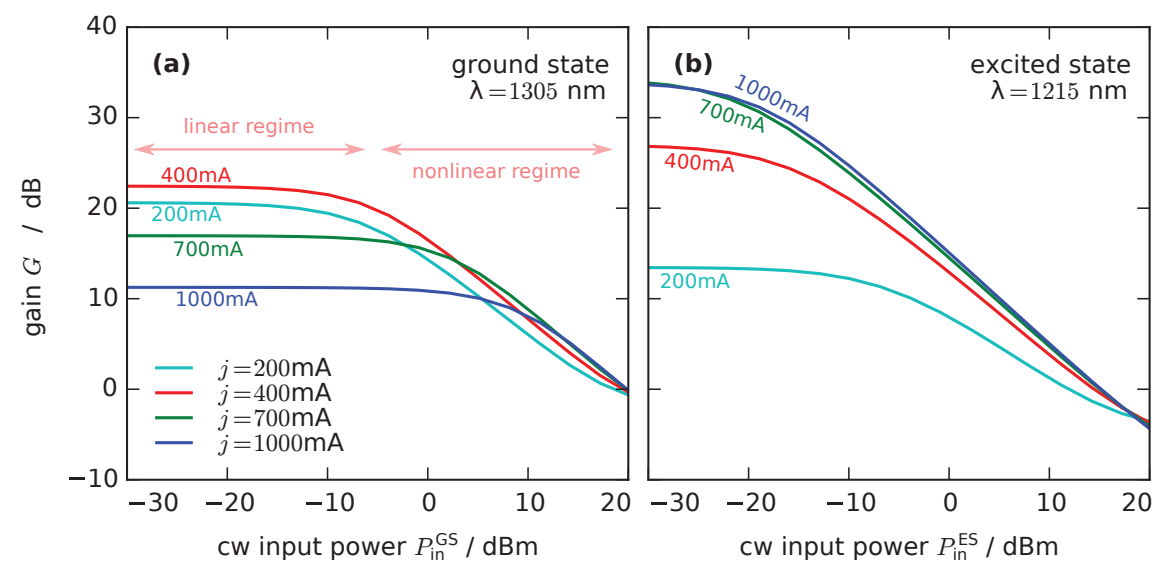

Figure 4.6.: Optical gain $G$ of the quantum-dot amplifier in dependence of the optical input power $P_{\mathrm{in}}^{m}$ for different pump currents $j$. Shown is the response to optical signals centered on (a) the ground-state, and (b) the excited-state frequency. The areas marked by the light red arrows denote the approximate extents of the linear and nonlinear amplification regimes for $j=400 \mathrm{~mA}$.

demands on the amplifier device are made. When a linear amplification of the input signal is required, the flat section of the gain characteristics should extend towards as high input power as possible, leading to a distortion-free amplification of arbitrarily strong optical signals [LAE06a]. On the other hand, optical signals with powers in the nonlinear regime induce a nonlinear response of the amplifier, which can be used, e.g., for wavelength conversion via four-wave-mixing [AKI02, QAS04, MAJ11a, SCH12e] or cross-gain-modulation [KIM09a, MEU10].

A general trend in the onset of the nonlinear regime towards higher optical power can be observed with increasing pump current in the ground-state. This effect can be explained by the increase of the in-scattering rates and the reservoir chargecarrier density [BER04]. The faster and more efficient refilling of the quantum-dot states after depletion by the optical signal shifts the saturation regime towards higher power. The gain at the excited-state wavelength, shown in Fig. 4.6 b, also shows the much higher gain compared to the ground-state. However, the onset of the nonlinear regime is located at much lower optical input power. This can be understood by the weaker confinement of the quantum-dot excited states compared to the ground state. This increases the sensitivity of the excited-state occupation to changes in the reservoir charge-carrier density. Together with the strongly increasing gain at the excited-state frequencies the saturation input power thus decreases with increasing pump current. 

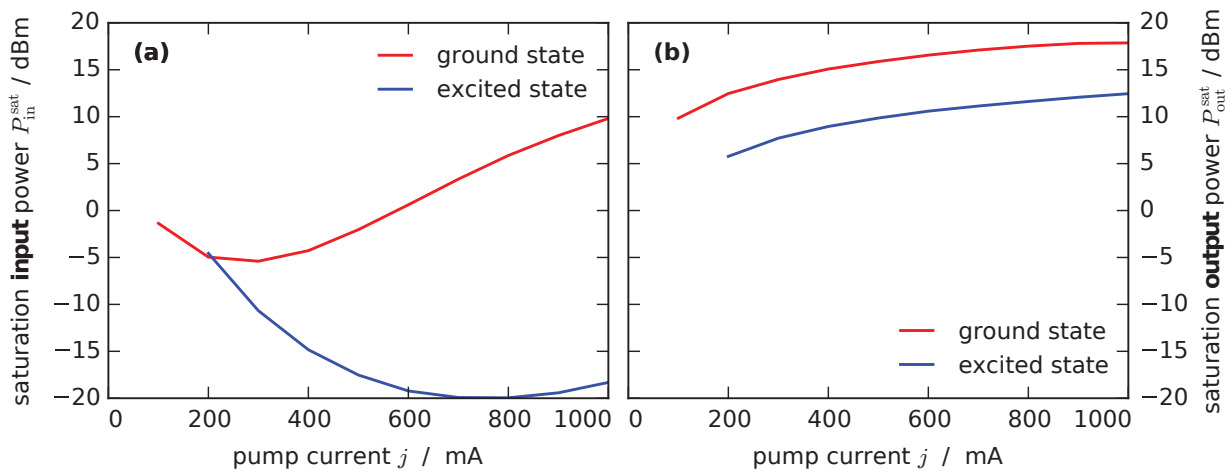

Figure 4.7.: Pump-current dependence of (a) the saturation input power and (b) the saturation output power of ground (red) and excited state (blue).

The pump-current dependence of the saturation input power is summarized in Fig. 4.7. Panel (a) quantifies the general trend that we have observed in Fig. 4.6. The ground-state saturation input power is minimal at $j=300 \mathrm{~mA}$ and then increases steadily, while for the excited state the minimum is reached at $j=800 \mathrm{~mA}$, and only then starts to increase again. This behavior shows the correlation with the optical gain, as a high gain will increase the internal optical power and thus decrease the input power required to induce a nonlinear response. Looking at the saturation output power $P_{\text {out }}^{\text {sat }}$, on the other hand, i.e., the output power for which the gain is reduced to $-3 \mathrm{~dB}$ relative to the small-signal gain, a steady increase with the pump current at both wavelengths can be seen. This is depicted in Fig. $4.7 \mathrm{~b}$. An increase in pump current therefore always increases the maximum output power for which near-linear amplification is possible [BER04, SCH05d, KIM08].

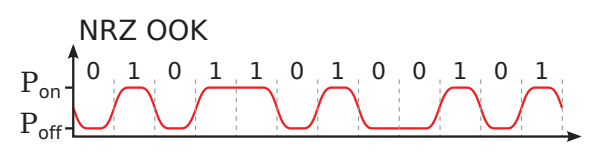

Figure 4.8: Illustration of the non-return-tozero pulse amplitude modulation scheme. The data stream is encoded by the power level, with $P_{\text {on/off }}$ corresponding to one and zero-bits, respectively.

\subsubsection{Amplification of Optical Data Streams}

We will now investigate the performance of the quantum-dot semiconductor optical amplifier under amplification of optical data signals. Optical data streams that are encoded via pulse-amplitude-modulation (PAM) use the signal power level to represent the data. For example, a bit-stream (zeroes and ones) can be encoded in a simple on-off scheme - "ones" are encoded as high power, "zeroes" as low power - referred to as on-off-keying (OOK) or PAM-2. In the following we will 


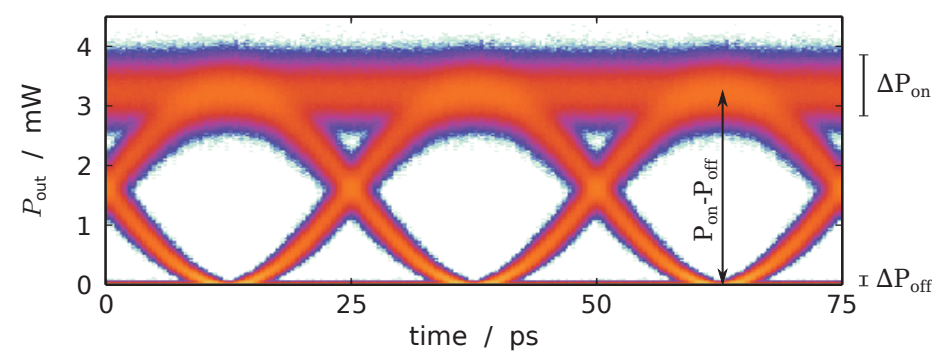

Figure 4.9.: Sketch of the evaluation of the quality factor from optical eye diagrams. Shown is an eye-diagram of the output optical data stream on the ground-state, for an optical input power of $P_{\mathrm{avg}}=-20 \mathrm{dBm}$ at $j=400 \mathrm{~mA}$. The quantities used in the evaluation of the Q-factor (Eq. (4.34)) are the standard deviations of the on and off-power-level, $\Delta P_{\text {on }}$ and $\Delta P_{\text {off }}$, respectively, and the eye-opening amplitude $P_{\text {on }}-P_{\text {off }}$.

consider non-return-to-zero (NRZ) OOK signals, as illustrated in Fig. 4.8. In the simulations, we will use optical data signals with a repetition rate of $40 \mathrm{~Gb} \mathrm{~s}^{-1}$. The input electric field is rewritten as

$$
E_{+}(0, t)=\left[P_{\mathrm{avg}}^{m} \frac{2}{A_{\mathrm{beam}} n_{\mathrm{bg}} \varepsilon_{0} c_{0}} \sum_{n_{\mathrm{on}}} A_{\mathrm{bit}}\left(t-t_{n_{\mathrm{on}}}\right)\right]^{\frac{1}{2}} e^{-i \omega^{m} t},
$$

where the index $n_{\text {on }}$ runs over the one-bits and $t_{n_{\mathrm{on}}}$ is the arrival time of the $n_{\mathrm{on}}{ }^{\text {th }}$ bit. The pulse amplitude shape is modeled as

$$
A_{\mathrm{bit}}(\tau)= \begin{cases}2 \cos \left(\frac{\pi}{2} f_{\mathrm{rep}} \tau\right)^{2} & |\tau| \leq \frac{1}{f_{\mathrm{rep}}} \\ 0 & |\tau|>\frac{1}{f_{\mathrm{rep}}}\end{cases}
$$

with the bit repetition rate $f_{\text {rep }}$. The above definition ensures that, for a data signal consisting of only one-bits, a constant optical power level is used as input. For a data signal with uniform distribution of zero and one-bits, the average optical input power is given by $P_{\mathrm{avg}}^{m}$. Here, we will employ bit-patterns with a random uniform distribution of zeroes and ones.

The results of the simulations for the amplification of an exemplarily chosen optical data stream in the quantum-dot semiconductor optical amplifier are presented in Fig. 4.9. It shows the resulting optical eye-diagram of the output signal, i.e., a histogram of the output power in time, created by overlaying the output of 240 bits. The eye diagram clearly shows the distinct zero and one power levels, i.e., a clear opening of the "eyes". Nevertheless, the power level of the one-bits, $P_{\text {on }}$, is 

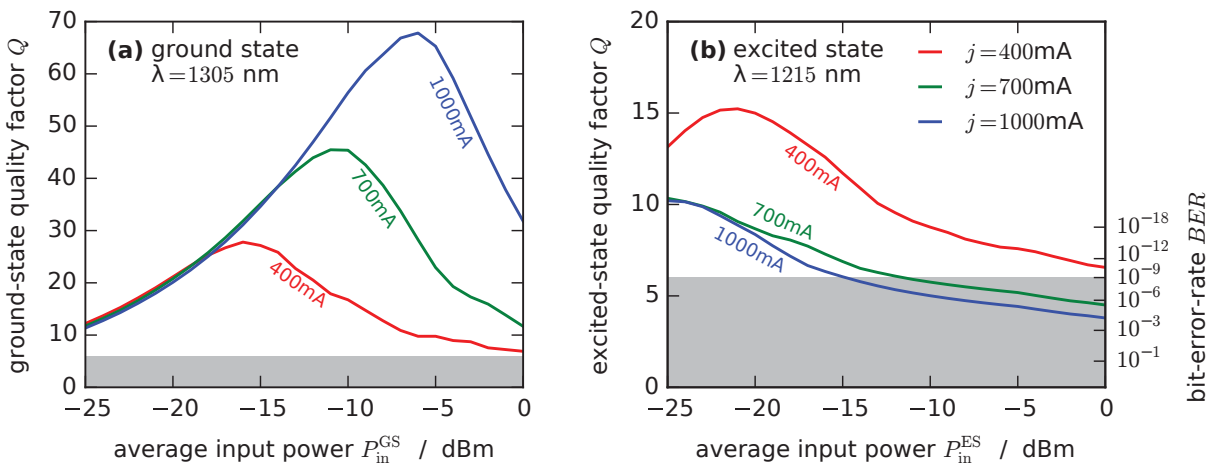

Figure 4.10.: Input-power dependence of the quality-factor for optical bit patterns at different pump currents. Shown is the quality factor of (a) signals centered at the ground-state $(\lambda=1305 \mathrm{~nm})$ and $(\mathrm{b})$ at the excited-state $(\lambda=1215 \mathrm{~nm})$, for pump currents of $j=400 \mathrm{~mA}$ (red), $700 \mathrm{~mA}$ (green), and $1000 \mathrm{~mA}$ (blue). The gray shaded area denotes the values of the $Q$-factor for which the $B E R$ is below $10^{-9}$. The input signals were non-return-to-zero on-off-keyed pseudo-random bit sequences with a $40 \mathrm{GHz}$ repetition rate.

rather noisy. In order to quantify the signal quality of the amplified data stream, we define the quality (or $Q-$ ) factor

$$
Q:=\frac{P_{\text {on }}-P_{\text {off }}}{\Delta P_{\text {on }}+\Delta P_{\text {off }}},
$$

with the variance $\Delta P$ of the corresponding on or off power level. The Q-factor thus describes the possibility to distinguish between the zero and one-levels in the optical data signal. For a low Q-factor the variance of the power would become larger, so a misinterpretation of the amplified data stream becomes more probable. This increases the bit-error-rate $(B E R)$, i.e., the ratio of wrongly decoded bits in the data stream. For a Gaussian distribution of the variation of the power levels from their mean, the bit-error-rate can be calculated from the Q-factor via [OLS89]

$$
B E R=\frac{1}{\sqrt{2 \pi} Q} \exp \left(-\frac{Q^{2}}{2}\right) .
$$

We employ the above definitions and calculate the quality factor for different pump currents in dependence on the average input power $P_{\text {avg }}$. The resulting curves are shown in Fig. 4.10 for signals on the ground and excited-state energies, respectively. The ground-state quality factor shows a clear maximum in the optical input power, for which the signal quality thus becomes optimal [WIL12c]. Towards lower input power the signal quality is limited by the spontaneous emission background noise, which becomes increasingly dominant for decreasing signal power. 

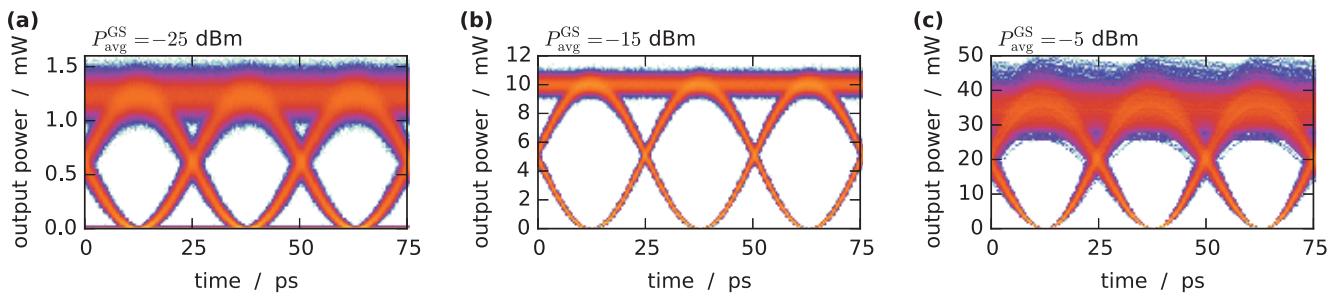

Figure 4.11.: Eye diagrams of the optical data signals on the ground-state after amplification for average input powers of (a) $P_{\mathrm{avg}}^{\mathrm{GS}}=-25 \mathrm{dBm}$, (b) $P_{\mathrm{avg}}^{\mathrm{GS}}=-15 \mathrm{dBm}$, and (c) $P_{\mathrm{avg}}^{\mathrm{GS}}=-5 \mathrm{dBm} . j=400 \mathrm{~mA}$, cf. Fig. 4.9 .

At high powers, on the other hand, the signal quality is limited by the onset of the nonlinear amplification regime, which introduces patterning effects in the output signal [BON11, WEG10]. This is depicted in Fig. 4.11, showing the transition from a noise-dominated signal at $P_{\mathrm{avg}}^{\mathrm{GS}}=-25 \mathrm{dBm}$, over a near-optical signal at $P_{\mathrm{avg}}^{\mathrm{GS}}=-15 \mathrm{dBm}$, to the appearance of patterning effects at $P_{\mathrm{avg}}^{\mathrm{GS}}=-5 \mathrm{dBm}$.

Consequently, we can observe an increase in the optimal input power with increasing pump current, correlated with the increasing saturation input power $P_{\text {in }}^{\text {sat }}$. For the excited-state, on the other hand, only for $j=400 \mathrm{~mA}$ a maximum in the quality-factor can be observed, whereas for higher currents the $Q$-factor steadily decreases with the input power and a general decrease of the quality is visible, due to the reduction of $P_{\text {in }}^{\text {sat }}$ we had seen in Fig. 4.7. Furthermore, the $Q$-factor is generally smaller than on the ground-state. This suggests that for signals on the excited-state frequencies, the signal quality is primarily limited by the onset of patterning effects, whereas spontaneous emission noise plays a smaller role.

Different demands on the bit-error-rate exist depending on the application and the use of error-correction techniques. Generally, a BER of $10^{-9}$ is favorable, corresponding to $Q \approx 6$. This requirement is met for all considered currents and input powers on the ground-state, and for $P_{\text {avg }}<-15 \mathrm{dBm}$ on the excited-state at $j=1000 \mathrm{~mA}$. Our results thus show that the given quantum-dot semiconductor amplifier can be effectively used to amplify data signals over a broad range of wavelengths.

While so far we have only considered the amplification of optical data streams centered on either of the quantum-dot state energies, a simultaneous amplification of two independent data signals might also be possible. We investigate this application in the next section. 


\subsection{Multi-State Operation of Quantum-Dot Amplifiers}

In the previous section, we have shown that the quantum-dot semiconductor optical amplifier can be used to amplify optical data streams corresponding to its ground-state and excited-state energies with good resulting signal quality. Subsequently, in this section we will investigate the possibility of a dual-state operation of the quantum-dot amplifier device, by simultaneous amplification of data signals on the ground and excited-state gain peaks. This would correspond, e.g., to the application of a single amplifier device for a dual-band amplification of counterpropagating upstream and downstream data signals on different wavelength bands. We will in the following perform simulations of the signal quality in dependence on the corresponding input power and the pump current.

We consider the simultaneous input of optical data signals centered on the ground and excited-state energies at opposing facets of the quantum-dot amplifier, as sketched in Fig. 4.12. We thus write, similar to Eq. (4.32), the boundary values of the electric field as

$$
\begin{aligned}
& E_{+}(0, t)=\left[P_{\mathrm{avg}}^{\mathrm{GS}} \frac{2}{A_{\text {beam }} n_{\mathrm{bg}} \varepsilon_{0} c_{0}} \sum_{n_{\mathrm{on}}^{\mathrm{GS}}} A_{\mathrm{bit}}\left(t-t_{n_{\mathrm{on}} \mathrm{GS}}\right)\right]^{\frac{1}{2}} e^{-i \omega^{\mathrm{GS}} t}, \\
& E_{-}(\ell, t)=\left[P_{\mathrm{avg}}^{\mathrm{ES}} \frac{2}{A_{\text {beam }} n_{\mathrm{bg}} \varepsilon_{0} c_{0}} \sum_{n_{\mathrm{on}}} A_{\mathrm{bit}}\left(t-t_{n_{\mathrm{on}}}\right)\right]^{\frac{1}{2}} e^{-i \omega^{\mathrm{ES}} t} .
\end{aligned}
$$

We thus look at the case that the ground-state signal is injected at the front facet $(z=0)$, and the excited-state signal at the back facet $(z=\ell)$. This counterpropagating setup reflects the application of a single optical amplifier device for the simultaneous amplification of an up and down-stream, from and to a telecommunication network node. Furthermore, the counter-propagation should minimize cross-talk between the two signals, whereas the individual bits in co-propagating signals could interact along the whole device length. Similar to the analysis done

Parts of this section have been published in [SCH15].

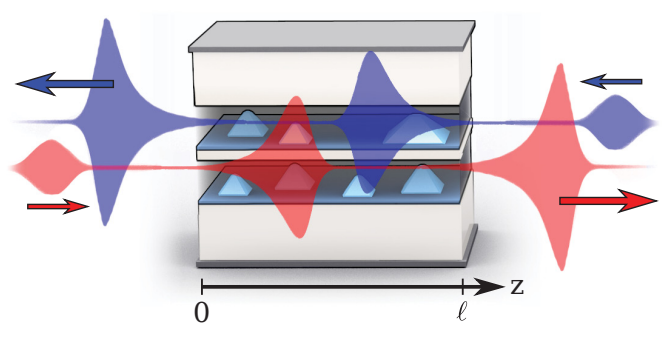

Figure 4.12: Sketch of the multi-state operation of the quantum-dot semiconductor optical amplifier. Optical signals centered on the ground (red) and excited-state (blue) frequencies are simultaneously injected into the amplifier through the front and back facets, respectively. 

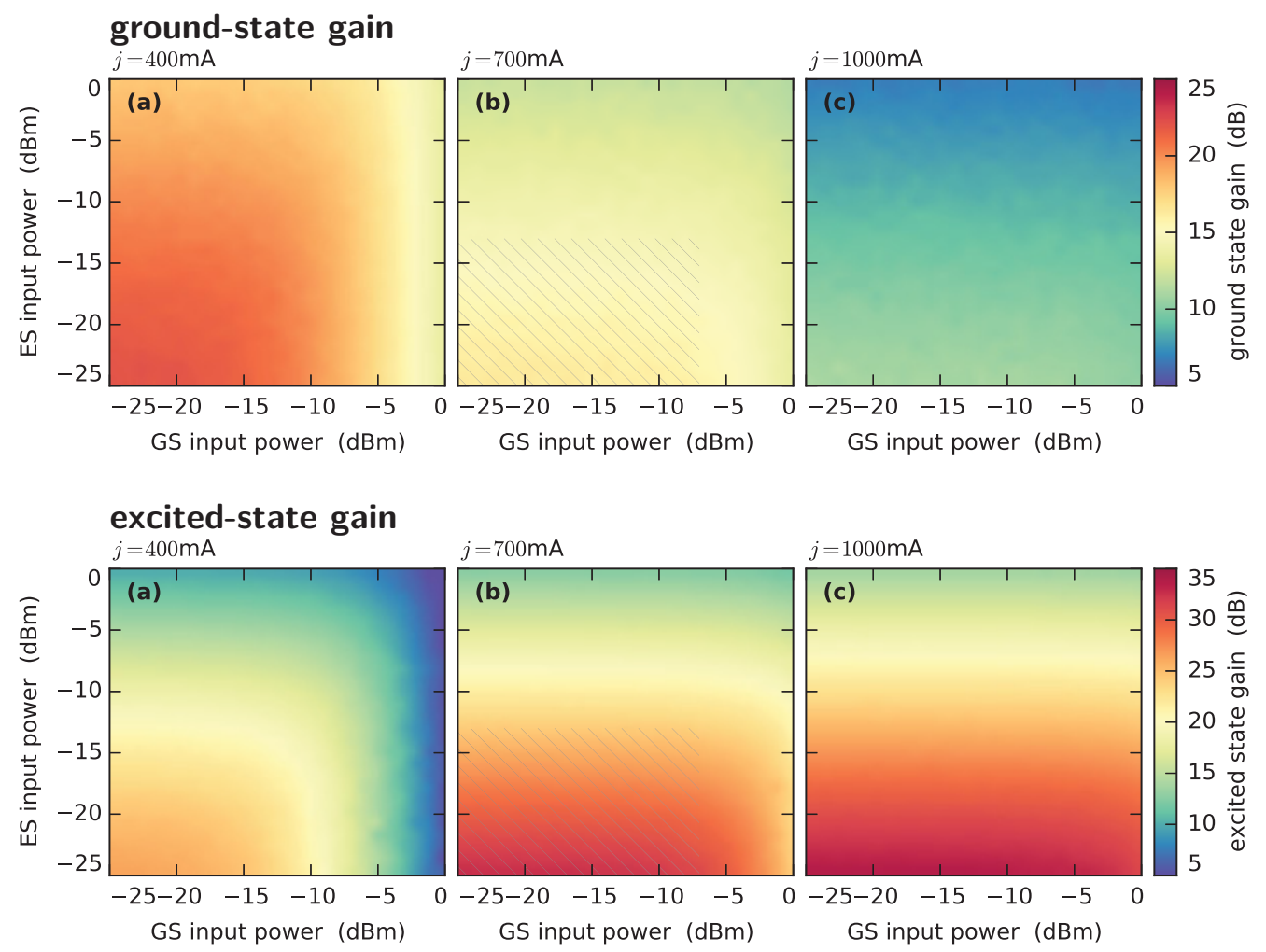

Figure 4.13.: Dependence of the ground-state (top row) and excited-state (bottom row) gain (color-coded) in dependence on the ground-state and excited-state average input powers for dual-state operation. Both for ground-state and excited-state a non-returnto-zero pseudo-random bit-sequence was used as input signal. Shown are the resulting values at (a) $j=400 \mathrm{~mA}$, (b) $j=700 \mathrm{~mA}$, and (c) $j=1000 \mathrm{~mA}$. The hatched area denotes the range of operation for which we expect good performance.

in the previous section we now analyze the dependence of the large-signal gain on the optical input power.

The top row in Fig. 4.13 shows the resulting large-signal gain for optical signals on the ground-state energy. For increasing pump current we can observe the decrease in gain that we have already seen previously. Also, the gain can be seen to decrease with increasing ground-state input power, once it reaches the saturation input power. Additionally, a steady decrease of the ground-state gain with increasing optical power on the excited state can be observed, which amounts to a $\approx 5 \mathrm{~dB}$ smaller gain when increasing the excited state input power from $-25 \mathrm{dBm}$ to $0 \mathrm{dBm}$ at the investigated current range. We can thus conclude that the excited-state amplification has only minuscule effect on the ground-state performance. 

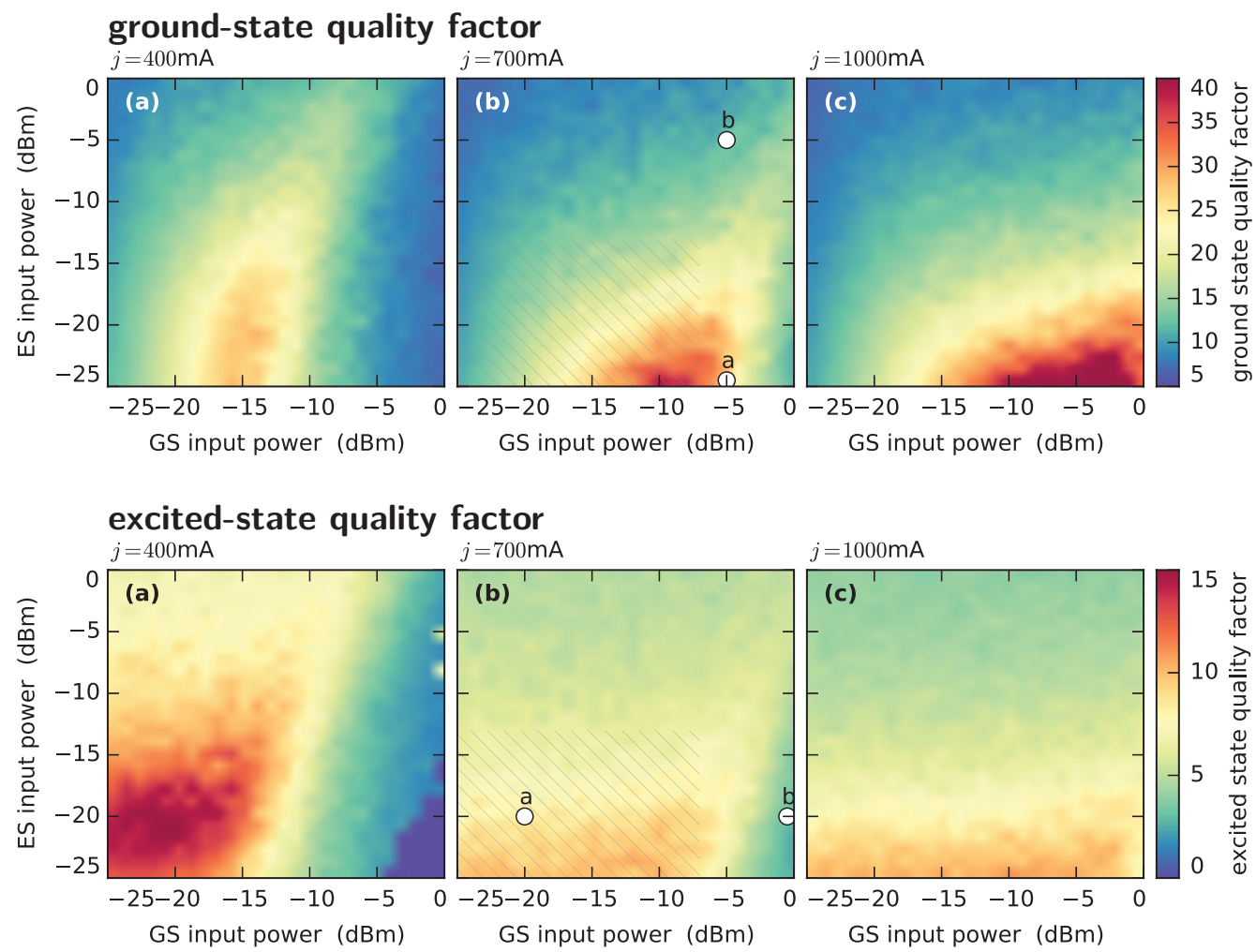

Figure 4.14.: Dependence of the ground-state (top row) and excited-state (bottom row) quality-factor (color-coded) in dependence on the ground-state and excited-state average input powers for dual-state operation. Both for ground-state and excited-state a non-return-to-zero pseudo-random bit-sequence was used as input signal. Shown are the resulting values at (a) $j=400 \mathrm{~mA}$, (b) $j=700 \mathrm{~mA}$, and (c) $j=1000 \mathrm{~mA}$. The hatched area denotes the range of operation for which we expect good performance. The labeled markers denote the operation parameters used in the respective panels in Fig. 4.15 (ground state) and Fig. 4.16 (excited state).

For the excited-state gain, shown in the bottom row in Fig. 4.13, on the other hand, a stronger influence of the ground-state signal can be seen at $j=400 \mathrm{~mA}$. Here, the excited-state gain decreases by $20 \mathrm{~dB}$ upon increasing the ground-state input power to $0 \mathrm{dBm}$. This can be understood by the intra-dot relaxation scattering driving the quantum-dot states towards quasi-equilibrium. As the quantum-dot ground-state is located at lower energy than the excited state, a variation of the excited-state occupation results in only little change of the quasi-equilibrium occupation at the ground-state. On the other hand, when the ground-state occupation is depleted by the optical signal, already a small change in its occupation will considerably impact the excited state population by the increased out-scattering into 

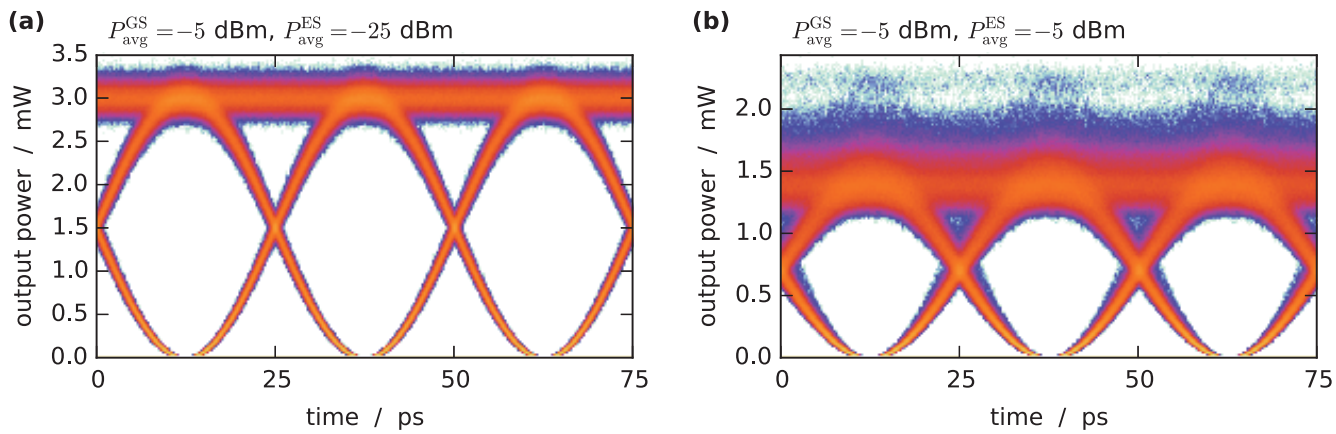

Figure 4.15.: Eye diagrams of the optical data signals on the ground-state under simultaneous amplification of an excited-state signal with (a) $P_{\mathrm{avg}}^{\mathrm{ES}}=-25 \mathrm{dBm}$, and (b) $P_{\mathrm{avg}}^{\mathrm{ES}}=-5 \mathrm{dBm}$. The ground-state optical input power in both cases is $P_{\mathrm{avg}}^{\mathrm{GS}}=-5 \mathrm{dBm}$. $j=700 \mathrm{~mA}$.

the now empty quantum-dot states. This explains the observed stronger impact of a ground-state signal on the excited state gain, as compared to the converse case.

For higher currents, however, not only the excited state gain is increased considerably, but also the perturbation by a ground-state signal is much less pronounced. This is a consequence of the increased reservoir carrier density, refilling the excited state more efficiently, and the decreasing gain of the ground-state, reducing the stimulated recombination rate on the ground-state. This shows that, in terms of the optical gain, the dual-state operation of the quantum-dot amplifier is in general possible. The pump-current must however be chosen adequately, to ensure low enough perturbation of the excited-state gain due to the presence of a signal on the ground state, while still providing strong enough ground-state gain. In our case, we therefore identify $j=700 \mathrm{~mA}$ as the optimal choice.

So far, we have only looked at the optical gain of the quantum-dot amplifier device under dual-state operation, showing promising results. The perturbation of the gain medium by the respective other data signal could however negatively impact the quality of the optical data streams. Analogously to the investigation of the optical gain in dependence of the optical input powers, we will therefore calculate the quality factor of the two simultaneously amplified signals. The resulting diagrams are shown in Fig. 4.14. The ground-state quality factor shows a clear maximum with respect to the ground-state input power, which is shifted towards higher ground-state power, when we additionally increase the optical power of the excited-state signal. This can be understood by the reduced optical gain on the ground-state that we had observed in Fig. 4.13. The increasing optical power on the excited state reduces the ground-state gain, which in turn reduces the signal-to-noise ratio of the ground-state signal for low input power. Further- 

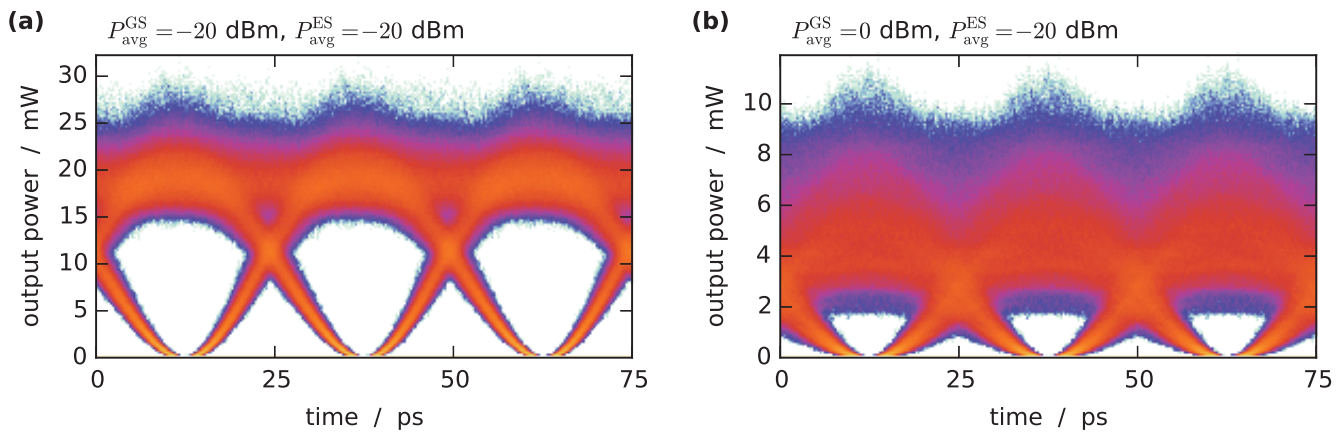

Figure 4.16.: Eye diagrams of the optical data signals on the excited-state under simultaneous amplification of a ground-state signal with (a) $P_{\mathrm{avg}}^{\mathrm{GS}}=-20 \mathrm{dBm}$, and (b) $P_{\mathrm{avg}}^{\mathrm{GS}}=0 \mathrm{dBm}$. The ground-state optical input power in both cases is $P_{\mathrm{avg}}^{\mathrm{ES}}=-20 \mathrm{dBm}$. $j=700 \mathrm{~mA}$.

more, a general decrease of the signal quality with increasing excited-state power can be observed. This is exemplarily shown in Fig. 4.15 for a ground-state input power of $P_{\mathrm{avg}}^{\mathrm{GS}}=-5 \mathrm{dBm}$, where for increasing power on the excited state a distortion of the ground-state eyes can be seen. The overall quality-factor, however, is well above 10 for most of the considered values, ensuring very good signal quality over a large parameter range.

With increasing pump current, the parameter range with a high $Q$-factor is extended towards higher ground-state input powers, leading to very little signal distortion at these currents. As we had seen before, this is however accompanied by a drastic reduction in the ground-state gain, which limits the applicability at high pump currents.

In the single-state operation on the excited-state, we had already observed a decrease of the overall signal quality on the excited state with increasing pump current. At the low current of $j=400 \mathrm{~mA}$, however, a severe deterioration of the excited-state signal quality with increasing ground-state power can be observed. This again shows the stronger perturbation of the excited-state gain caused by variations of the ground-state occupation, exemplarily illustrated in Fig. 4.16. Here, the increasing ground-state intensity leads to a strong distortion of the one-level. Nevertheless, it can still be clearly distinguished from the zero-level. A higher pump current can partly alleviate this distortion and reduce the reduction in the Q-factor due to the presence of a ground-state signal.

Taking into account the signal quality as well as the optical gain, we predict best performance under dual-state operation for a pump current around $700 \mathrm{~mA}$ and input powers of up to $P_{\mathrm{in}}^{\mathrm{GS}} \leq-7 \mathrm{dBm}$ and $P_{\mathrm{in}}^{\mathrm{ES}} \leq-13 \mathrm{dBm}$. This parameter region is denoted in Figs. 4.13 and 4.14. 
To conclude, we investigated the possibility of a dual-state operation of a quantumdot semiconductor optical amplifier for amplification of optical data signals. We have considered the simultaneous amplification of counter-propagating optical nonreturn-to-zero on-off-keyed data signals, in frequency bands centered on the groundstate and excited-state gain peaks, respectively. We have adjusted our model parameters by comparison with experimental data and achieved good quantitative agreement. Our results predict the possibility to use the same quantum-dot amplifier device for simultaneous amplification of counter-propagating optical data streams while providing sufficient gain and a high enough signal quality on both frequency bands. In general the excited-state signal quality was found to be more prone to the perturbation by the presence of a ground-state signal, as compared to the converse case. A proper choice of the pump current, in our case $j=700 \mathrm{~mA}$, yields strong enough gain in the ground state and good signal quality for signals on the excited state to make the dual-state operation possible. 


\subsection{Coherent Transients in Quantum-Dot Amplifiers}

Coherence in light-matter interaction refers to physical phenomena in which the phase between the active medium polarization and the incident light field is maintained. A precondition for such effects is thus a sufficiently long lifetime of the microscopic polarization, i.e., a slow dephasing. Investigations of this topic are therefore often limited to systems at low temperature and with only few emitters [FRY93, SCU97, PHI01, BRI10], in order to keep the number of possible dephasing processes small [CHO03].

Semiconductors were consequently initially disregarded as potential candidates for the observation of coherent transients and quantum-coherent interaction, due to the high density of charge-carriers [HAU89, SAY94] and the dephasing due to scattering with lattice phonons [SCH86h, FAN98]. Furthermore, the superposition of an ensemble of optical transitions with different energies leads to an additional decay of the polarization [SHA96]. On the other hand, with the advent of semiconductor quantum dots, atom-like transitions within the semiconductor material could be realized. Semiconductor quantum-dot transitions were shown to exhibit much longer dephasing times [GAM96, BOR01a], making localized quantum-dots an attractive choice for applications in quantum-optics [BRA05].

Recently, however, coherent pulse propagation in macroscopic semiconductor devices have been observed in quantum-cascade lasers [CHO10], quantum-dash [CAP14] and quantum-dot semiconductor amplifiers [KOL13], relying on ultra-short, strong optical pulses. It is important to note that in the experiments using optical amplifiers, the measurements were performed at room temperature, opening up possible future quantum-coherent applications using uncooled devices.

In this section, we will present experimental measurements and a theoretical description of Rabi oscillations induced by ultra-short pulses in a quantum-dot semiconductor optical amplifier at room temperature [KOL13]. Rabi oscillations denote a periodic exchange of energy between the optical field and the active

Parts of this section have been published in [KOL13].

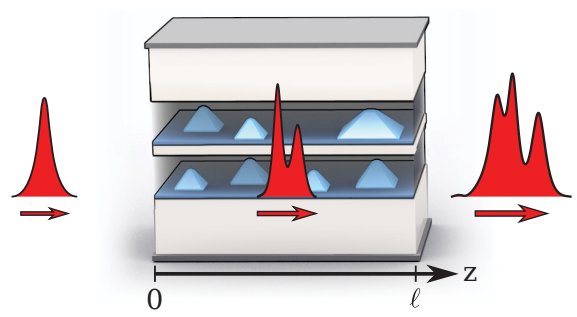

Figure 4.17: Sketch of the Rabioscillation induced pulse shaping in quantum-dot semiconductor optical amplifiers. The strong and ultra-short input pulse excited Rabi oscillations in the active medium, which lead to the formation of characteristic dips and peaks in the optical pulse. 
medium, which leads to characteristic modifications of the optical pulse traveling through the amplifier device, as sketched in Fig. 4.17. Apart from the appearance of Rabi oscillations, the long dephasing in semiconductor quantum-dots can enable quantum-coherent effects such as lasing without inversion [SCU89, HAR89] and electromagnetically-induced transparency [MCC69, BOL91, HAR97c]. The observation of quantum-coherence in semiconductor devices might therefore open the possibility of new applications utilizing the coherent interaction between active medium and light [CHO13a].

In this section we will present simulations of the propagation of ultra-short pulses through a quantum-dot semiconductor optical amplifier. We investigate the pulseamplitude dependent pulse shaping by Rabi oscillations and show that the coherent signature can in principle be observed by measurements of the small-signal gain. Then, we compare our theoretical results with electric-field cross-correlation measurements in a pulse-propagation setup, and we will show that the experimentally observed pulse shaping is indeed the result of Rabi oscillations.

\subsubsection{Rabi-Oscillations in Quantum-Dot Semiconductor Amplifiers}

The quantum-dot semiconductor optical amplifier device we are modeling in this section consists of a $2 \mu \mathrm{m}$ wide and $1.5 \mathrm{~mm}$ long shallow-etched waveguide. The active medium is a DWELL structure composed of 15 layers of InGaAs quantumwells with embedded InAs quantum-dots. The model parameters used to describe the device are given in Table 4.2. In order to describe the dynamic dependence of the dephasing time on the pump current, we implement the following fit function:

$$
T_{2}\left(w_{e}, w_{h}\right)=\left[3 \mathrm{ps}^{-1}+\left(\frac{w_{e}+w_{h}}{4.4 \cdot 10^{11} \mathrm{~cm}^{-2}}\right)^{0.92} \mathrm{ps}^{-1}\right]^{-1}
$$

The first term accounts for dephasing processes, e.g., due to phonon-carrier scattering, which we assume to be independent of the surrounding charge-carrier density. Here we take a dephasing rate of $3 \mathrm{ps}^{-1}$ to account for these effects. It has been shown theoretically [BIN92, KOC00, VU06, LOR06, KOP11, GOL14] and experimentally [BOR02] that the dephasing time in a DWELL structure depends on the charge-carrier density in the carrier reservoir. We thus implement a numeric fit to the carrier-density dependence of the dephasing time presented in [KOP11], leading to the second term in Eq. (4.37).

Compared to the quantum-dot amplifier device investigated in the previous sections, a shallower quantum-dot confinement is assumed here. The dependence of the dephasing time on the reservoir charge-carrier density of both devices is plotted in Fig. 4.18. While the qualitative dependence is the same, the shallower quantum- 
Table 4.2.: Parameters used in the simulations in section 4.5, unless stated otherwise.

\begin{tabular}{lll}
\hline \hline SYMBOL & VALUE & MEANING \\
\hline$N^{\mathrm{QD}}$ & $3 \times 10^{10} \mathrm{~cm}^{-2}$ & QD density per layer \\
$a_{L}$ & 15 & number of layers \\
$h^{\mathrm{QW}}$ & $4 \mathrm{~nm}$ & QW layer height \\
$n_{\mathrm{bg}}$ & 3.77 & background index \\
$\Delta E_{\mathrm{inh}}$ & $30 \mathrm{meV}$ & QD inhomogeneous broadening FWHM \\
$A^{S}$ & $0 \mathrm{~ns}^{-1}$ & QW linear recombination rate \\
$B^{S}$ & $540 \mathrm{~nm}^{2} \mathrm{~ns}^{-1}$ & QW bimolecular recombination rate \\
$W_{\mathrm{GS}}$ & $0.44 \mathrm{~ns}^{-1}$ & GS spontaneous recombination rate \\
$W_{\mathrm{ES}}$ & $0.55 \mathrm{~ns}^{-1}$ & ES spontaneous recombination rate \\
$\mu$ & $0.6 \mathrm{~nm} e_{0}$ & QD transition dipole moment \\
$T_{2}$ & see Eq. $(4.37)$ & QD polarization dephasing time \\
$\Gamma$ & $0.1+\frac{J}{600 J_{0}}$ & Geometric confinement factor \\
$\Delta E_{e}\left(\Delta E_{h}\right)$ & $74 \mathrm{meV}^{-1}(50 \mathrm{meV})$ & electron (hole) QD GS localization energy \\
$\Delta_{e}\left(\Delta_{h}\right)$ & $40 \mathrm{meV}^{(20 \mathrm{meV})}$ & electron (hole) QD GS-ES energy spacing \\
$Z$ & 30 & number of space discretization steps \\
\hline \hline
\end{tabular}

dot confinement leads to a longer dephasing time, in agreement with microscopic calculations [LOR06b].

In this section we limit ourselves to a single forward-propagating electric field pulse, and neglect spontaneous emission noise for simplicity. We therefore need to describe only one electric field variable, which we label $E(z, t)$. The input pulse is assumed to enter the amplifier device at $z=0$, with the back facet at $z=\ell$. Furthermore, noise effects are neglected, as the shorter length of the amplifier device leads to a lower noise intensity. Additionally, optical signals with much higher peak power compared to the previous sections are considered here, which makes amplified spontaneous emission noise negligible.

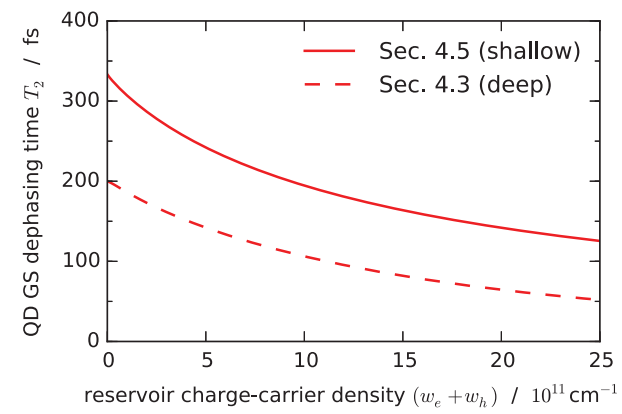

Figure 4.18: Fitted dephasing times for the quantum-dot semiconductor optical amplifier devices in Sec. 4.3 (deep confinement, dashed) and here (Sec. 4.5, shallow confinement, solid). Shown is the groundstate dephasing time in dependence of the total reservoir charge-carrier density $w_{e}+w_{h}$, 
In order to understand the emergence of Rabi oscillations, we take a look at the dynamic equation for a single quantum-dot subgroup:

$$
\begin{gathered}
\frac{\partial}{\partial t} p_{m}^{j}(z, t)=-\left[i\left(\omega_{m}^{j}-\omega\right)+\frac{1}{T_{2}}\right] p_{m}^{j}-i \frac{\mu_{m}}{2 \hbar}\left(\rho_{e, m}^{j}+\rho_{h, m}^{j}-1\right) E(z, t), \\
\frac{\partial}{\partial t} \rho_{b, m}^{j}(z, t)=-\frac{1}{\hbar} \operatorname{Im}\left[p_{m}^{j} \mu_{m}^{*} E^{*}(z, t)\right]-W_{m} \rho_{e, m}^{j} \rho_{h, m}^{j}+\left.\frac{\partial}{\partial t} \rho_{b, m}^{j}\right|_{\mathrm{sc}} .
\end{gathered}
$$

We reduce the above equations by introducing the inversion $d:=\left(\rho_{e, m}^{j}+\rho_{h, m}^{j}-1\right)$ and neglecting losses. Furthermore, we limit ourselves to a resonant excitation, i.e., $\omega=\omega_{m}^{j}$ :

$$
\begin{aligned}
& \frac{\partial}{\partial t} p_{m}^{j}(z, t)=-i \frac{\mu_{m}}{2 \hbar} d(z, t) E(z, t), \\
& \frac{\partial}{\partial t} d(z, t)=-\frac{2}{\hbar} \operatorname{Im}\left[\mu_{m}^{*} E^{*}(z, t) p_{m}^{j}(z, t)\right] .
\end{aligned}
$$

For an initial inversion of $d_{0}$ and vanishing polarization amplitude, the above equations have the solution

$$
\begin{aligned}
p_{m}^{j}(z, t) & =-\frac{i}{2} \sin \Theta(z, t), \\
d(z, t) & =d_{0} \cos \Theta(z, t),
\end{aligned}
$$

with the pulse area defined as

$$
\Theta(z, t)=\int_{-\infty}^{t} \frac{\mu_{\mathrm{GS}}}{2 \hbar}\left|E\left(z, t^{\prime}\right)\right| \mathrm{d} t^{\prime} .
$$

Equation (4.40b) shows that, with a proper choice of the pulse area, it is possible, e.g., to invert the charge-carrier distribution by choosing $\Theta=\pi$. When choosing $\Theta=n 2 \pi, n \in \mathbb{N}$, i.e., integer multiples of $2 \pi$, the system returns to its initial state after the exciting pulse has passed through. This important property shows that the coherent interaction between the optical field and the active material is a reversible process, as long as the polarization dephasing time is larger than the pulse width. The $\approx$ ps long pulses that we have investigated in Sec. 4.3 , on the other hand, were significantly longer than the dephasing time. Their interaction with the active medium was thus irreversible.

We now use our quantum-dot semiconductor optical amplifier model to simulate the amplification of ultra-short optical pulses. Here, we consider Gaussian pulses 

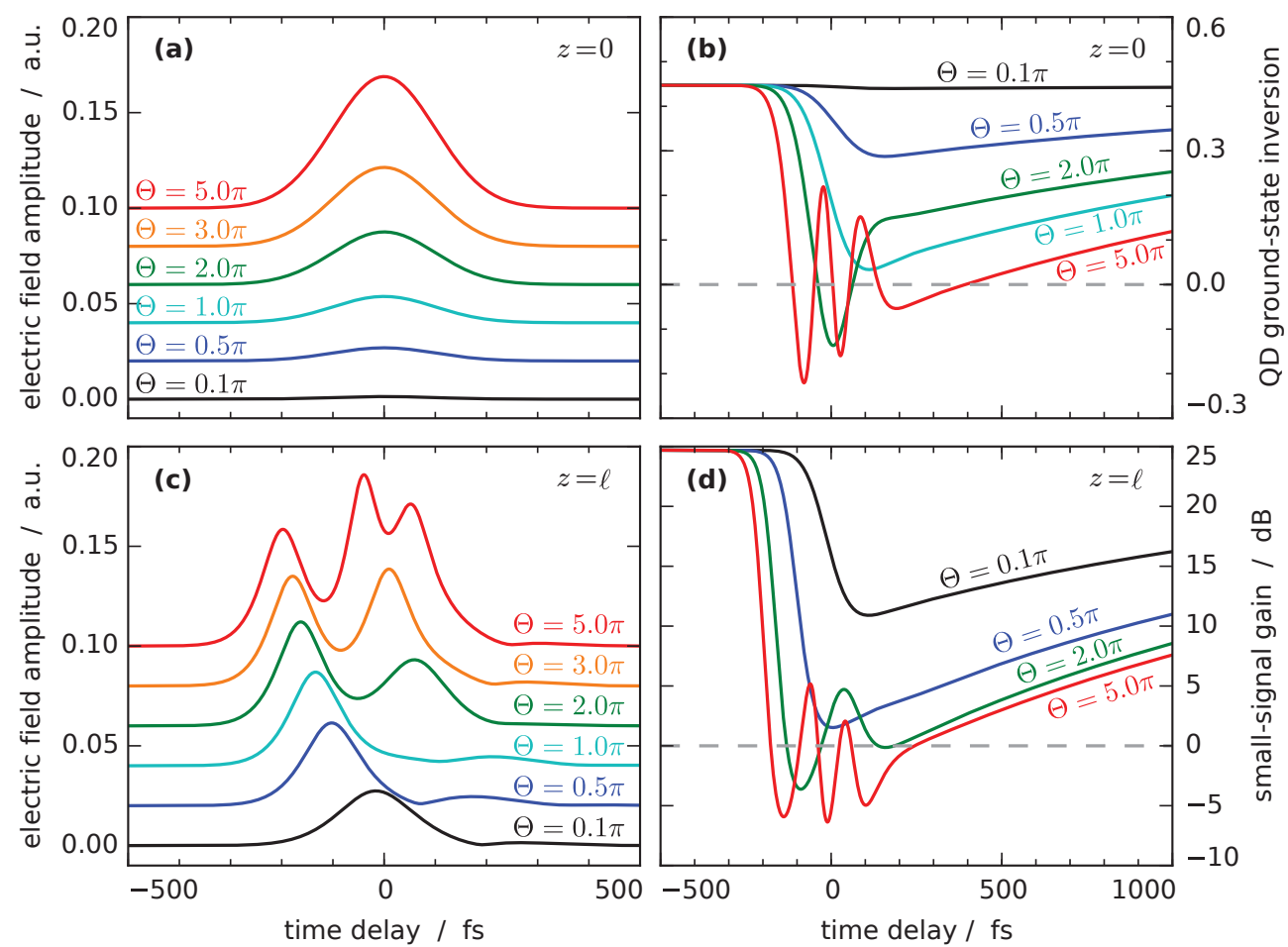

Figure 4.19.: Rabi-oscillations in the quantum-dot semiconductor optical amplifier. (a) Input electric field amplitudes for different input pulse areas $\Theta$. (b) Corresponding time-traces of the quantum-dot ground-state inversion of the resonant subgroup at the input facet for different input pulse areas. (c) Electric field amplitudes at the outputfacet for different input pulse areas $\Theta$. (d) Corresponding time-trace of the integrated small-signal gain, Eq. (4.43), for different input pulse areas. The curves in (a),(c) are shifted along the vertical axis for better readability. The time delay is given with respect to the arrival time of the pulse at the corresponding device facet. $J=20 J_{0}$.

with a width of $\delta t=235 \mathrm{fs}$ (FWHM) in amplitude. Taking the optical pulse area $\Theta$ as an input parameter, the input electric field is thus given by

$$
E(0, t)=\Theta \frac{2 \hbar}{\mu_{\mathrm{GS}}} \frac{\sqrt{4 \log 2}}{\delta t \sqrt{\pi}} \exp \left[-4 \log 2\left(\frac{t-t_{0}}{\delta t}\right)^{2}\right] e^{-i \Delta \omega t},
$$

where $t_{0}$ is the pulse arrival time at the front facet. The detuning from the optical carrier frequency $\Delta \omega$ is chosen to yield a center frequency of the optical pulse corresponding to a blue-shift of $6 \mathrm{meV}$ from the quantum-dot ground-state gain maximum, in accordance with the experimental setup discussed in Sec. 4.5.2.

Fig. 4.19 a shows the electric field amplitude of the pulse that enters the input facet of the amplifier for different values of the input pulse area $\Theta$. The amplifier 
is biased at twenty times the transparency current density $J_{0}$. The transparency current density is defined as the current density at which the ground-state gain is $0 \mathrm{~dB}$, i.e., no net amplification (or loss) along the device is observed. At $J=20 J_{0}$ the initial ground-state inversion is approximately 0.45 , as can be seen in Fig. $4.19 \mathrm{~b}$ prior to the pulse arrival. For $\Theta=0.1$ the inversion can be seen to be nearly unaffected by the input pulse, as the input power and the corresponding stimulated emission rate is too low to disturb the inversion appreciably. With increasing pulse area, a clear reduction in the ground-state inversion is visible, with a subsequent refilling by the charge-carrier scattering processes.

For an input pulse area of $\Theta=2 \pi$, shown by the green line, the ground-state inversion reaches a minimum value well below zero. This is a clear indication of Rabi-oscillations and thus evidence of coherent interaction within the device. From Eq. (4.40b), we would expect a complete Rabi oscillation for the input pulse area of $2 \pi$, i.e., the inversion should reach its original value after the pulse has passed. This expectation is not fulfilled, due to the presence of dephasing processes and a finite charge-carrier lifetime, which lead to a damping of the Rabi oscillations [SCU97]. This is especially visible for $\Theta=5 \pi$, which clearly show two and a half damped Rabi cycles.

The corresponding output pulses, shown in Fig. 4.19 c, exhibit the appearance of characteristic modifications of the pulse shape with increasing pulse area. While the input area of $0.1 \pi$ is too low to induce Rabi oscillations, resulting in a nearly Gaussian-shaped output pulse, the higher pulse areas lead to the formation of additional peaks in the output field amplitude.

The signature of the Rabi oscillations is also visible in the small-signal device gain, shown in Fig. $4.19 \mathrm{~d}$, which is calculated via

$$
G(\tau)=\exp \left[2 \int_{0}^{\ell} \mathrm{d} z \frac{1}{v_{g}} \operatorname{Re} g\left(z, t_{0}+\frac{z}{v_{g}}+\tau\right)\right],
$$

where $g(z, t)$ is the complex gain as defined in Eq. (2.75). Equation (4.43) describes the intensity gain that a small pulse traveling along the amplifier waveguide would experience, with $\tau$ denoting the time-difference between this virtual pulse and the probe pulse. $G(\tau)$ thus describes the gain measured in pump-probe experiments [DOM07, GOM09, MAJ11]. Thus, with a sufficiently narrow probe pulse and high time resolution, the signature of Rabi oscillations should be possible to detect in a pumpprobe setup.

The theoretical results clearly show the possibility of coherent interaction in the form of Rabi oscillations in quantum-dot semiconductor optical amplifiers. In the 


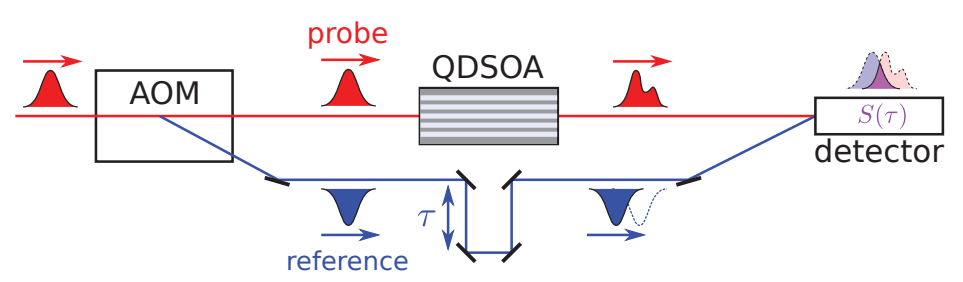

Figure 4.20.: Simplified sketch of the heterodyne measurement technique. The incoming optical pulse is split into two beam paths in an acousto-optic modulator (AOM). The probe pulse (red) passes through the quantum-dot semiconductor amplifier (QDSOA), while the reference pulse (blue) goes through a variable delay-stage. The two pulses are then lead to the detector (lock-in-amplifier), where the cross-correlation between the delayed input pulse and the amplified pulse is measured.

next section, we will thus compare our model with experimental cross-correlation measurements of ultra-short pulses in a quantum-dot amplifier.

\subsubsection{Comparison with Experimental Measurements}

We now apply the quantum-dot amplifier model to recreate the results from an cross-correlation experimental setup. Since the electric field amplitude is easily accessible in our model, but is not immediately accessible by experimental detection schemes, different measurement techniques must be employed. Here, we compare our theoretical results with heterodyne cross-correlation measurements ${ }^{1}$ (FROSCH: frequency-resolved optical short-pulse characterization by heterodyning [KOL13]).

A simplified sketch of the heterodyning setup is shown in Fig. 4.20. An incoming short optical pulse is first split into two separate beam paths by an acousto-optic modulator, which also induces a frequency shift in the extraordinary (reference) beam. The ordinary (probe) beam is coupled into the quantum-dot amplifier, while the reference pulse goes through a variable delay stage. The delayed reference beam and the probe beam are recombined onto a lock-in amplifier, detecting the beat note between the pulses. The measured signal $S(\tau)$ is thus proportional to the cross-correlation of the reference and probe pulses:

$$
S(\tau) \propto\left|\int_{-\infty}^{\infty} \mathrm{d} t E_{\mathrm{p}}(t) E_{\mathrm{r}}^{*}(t+\tau)\right|,
$$

where $E_{\mathrm{r}}$ and $E_{\mathrm{p}}$ are the reference and probe pulse electric fields, respectively. The advantage of this technique is the high temporal resolution of below $1 \mathrm{fs}$, since the

\footnotetext{
${ }^{1}$ The measurements were performed by Mirco Kolarczik, Nina Owschimikow, and Yücel Kaptan, Inst. f. Optik u. Atomare Physik, TU Berlin.
} 
$\Theta=0.33 \pi$

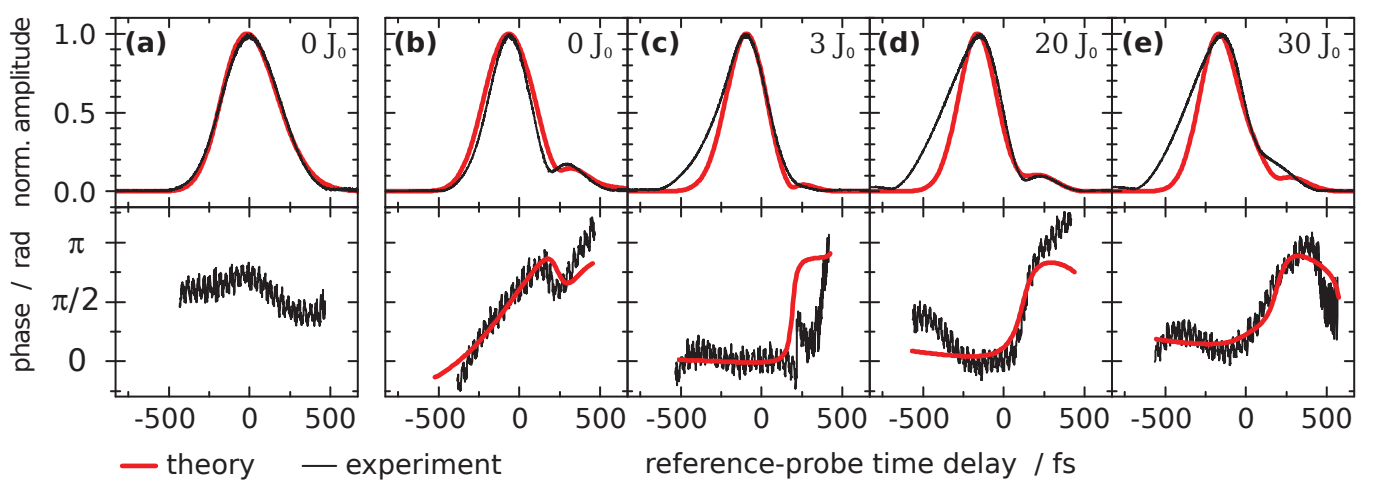

Figure 4.21.: Comparison of the Rabi-oscillations in the amplified ultra-short pulses between experiment and theory. Shown is the normalized cross-correlation amplitude, Eq. (4.44), (top panel) and its phase (bottom panel), both for the experimental results (black) and theoretical predictions (red), as a function of the delay $\tau$ between probe and reference pulses. (a) Low input power of $1 \mathrm{~mW}(\Theta=0.33 \pi)$ and $J=0$. (b)-(e) High input power of $12 \mathrm{~mW}(\Theta=1.15 \pi)$ for current densities of $J=0, J=3 J_{0}, J=20 J_{0}$, and $J=30 J_{0}$, respectively. Modified from [KOL13].

time-delay $\tau$ can be precisely adjusted. Furthermore, the measured signal is linear in the probe amplitude $E_{\mathrm{p}}$, providing high sensitivity even at low light intensities. On the other hand, a drawback of the cross-correlation is that the electric field itself cannot be measured independently. Only the convolution of the probe and reference pulses is accessible. Thus, while the signal $S(\tau)$ has a high resolution in $\tau$, the achievable resolution in $t$ of the actual electric field amplitude $E_{\mathrm{p}}(t)$ is limited by the width of the reference pulse.

While Eq. (4.44) does not allow for a direct access to the electric field amplitude, it is still closely related to the electric field pulse shape. For example, the crosscorrelation of two Gaussian pulses will again yield a Gaussian pulse in $\tau$, albeit with a larger width. In addition to the cross-correlation amplitude, also the phase of the correlation integral in Eq. (4.44) can be experimentally determined. This allows the evaluation of time-resolved phase dynamics of the amplified pulse, and can be used, e.g., to determine charge-carrier induced frequency chirp [VAL08].

We proceed by comparing the measured pump-current dependent pulse amplification in the quantum-dot amplifier with our theoretical results. In the experiments, input pulses with widths of $\delta t=235 \mathrm{fs}$ with variable pulse area are produced by an optical parametric oscillator with a repetition rate of $75.4 \mathrm{MHz}$. The measurements presented here were obtained at room-temperature. The resulting cross-correlation curves are shown in Fig. 4.21, where we compare both the cross-correlation amplitude and phase with the experimental results. Fig. 4.21 a shows the resulting curves 
for an input pulse area of $\Theta=0.33 \pi$ and an unpumped, i.e., absorbing, device. The output pulse shape is nearly Gaussian, which is to be expected from a low input pulse area, as we have already seen in the previous section. Upon increasing the input pulse area to $\Theta=1.15 \pi$, shown in Fig. $4.21 \mathrm{~b}$-e, modifications to the pulse shape become visible. These are most pronounced at $J=0$ and $J=20 J_{0}$, i.e. for empty and moderately filled quantum-dots, where a pronounced dip and additional peak in the amplitude for positive time delays can be observed. At $J=3 J_{0}$, only minor pulse shape modifications are visible, due to operating near transparency. For even higher pump currents, $J=30 J_{0}$, while the initial quantum-dot inversion is increased, the dephasing time of the optical transitions is much lower due to the high number of injected charge carriers. This leads to a less pronounced coherent interaction, and the amplitude dip is much smaller. The investigation of the cross-correlation phase reveals that the appearance of the minima in the amplitude is accompanied by a phase jump, due to the change of sign of the electric field induced by the Rabi oscillations [SCU97].

The theoretical results reproduce the experimental findings very well. We can therefore conclude that our model is well suited for the description of the quantumdot amplifier device. However, we can also use the model to investigate the dynamics of the amplifier during the coherent interaction, which cannot be resolved experimentally.

Figure 4.22 shows the quantum-dot ground-state dynamics at the input and output facets for the amplification of a pulse with $\Theta=1.15 \pi$ at $J=20 J_{0}$, as already shown in Fig. 4.21 d. In Fig. $4.22 \mathrm{a}$,b the corresponding cross correlation amplitudes, along with the solitary electric field amplitude, are shown. Here, the slight loss of detail due to the evaluation of the cross-correlation can be observed, as the electric field amplitude at the output facet shows a more complex shape than the resulting cross-correlation signal. The cross-correlation translates this complex shape into a smooth envelope with a single amplitude dip.

The color-coded density plots shown in Fig. $4.22 \mathrm{c}$, d depict the time-evolution of the polarization amplitude of the different quantum-dot subgroups, characterized by their detuning with respect to the pulse center frequency. Here it can be seen how the incoming pulse at first builds up a polarization in all quantum-dot subgroups, albeit with a much stronger response at the resonant subgroup. The dynamics at the front facet, panel c, already exhibits a difference in the dynamics of the different quantum-dot subgroups, with a more persistent polarization for subgroups slightly detuned from the pulse. This becomes even more pronounced at the output facet, where the pulse shape is more complex and the pulse area higher due to the amplification along the amplifier. Here, the dynamics of the individual quantumdot subgroups is much more complex, and the Rabi oscillations faster due to the increased field amplitude. The polarization dynamics is not symmetric around 


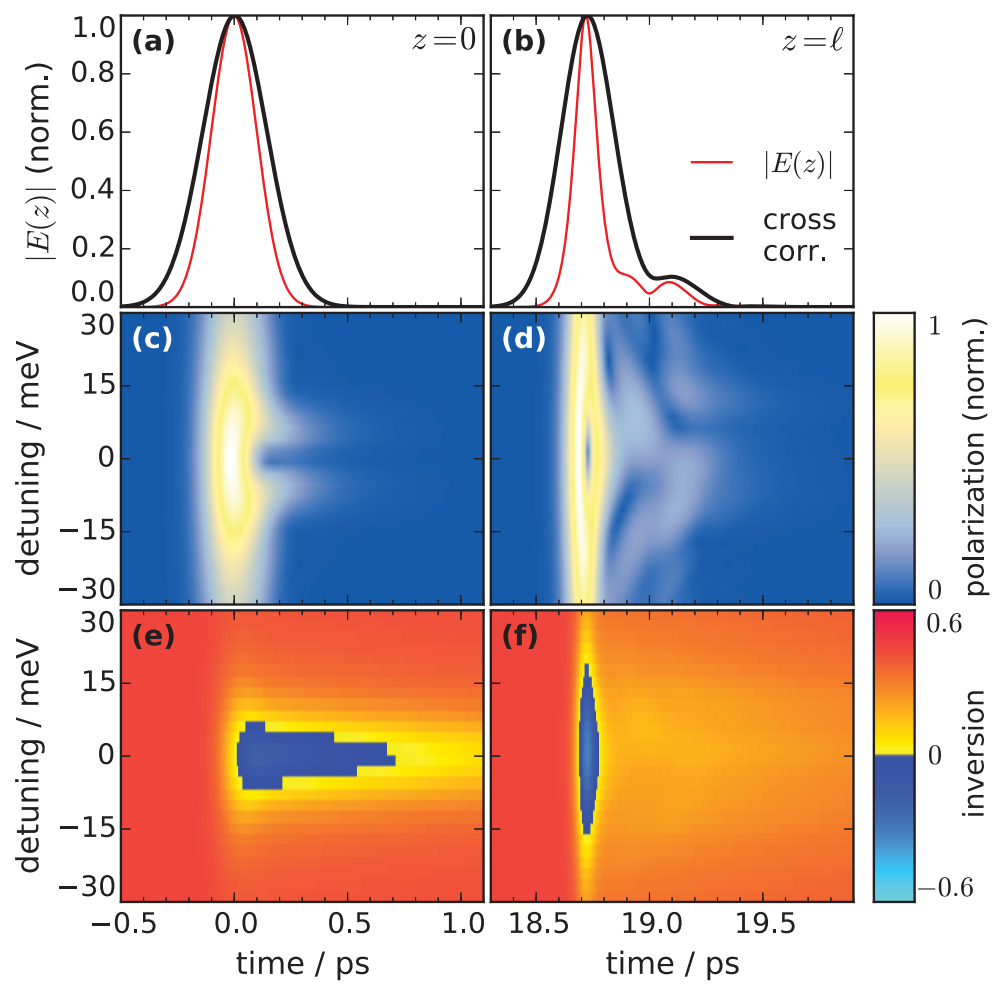

Figure 4.22.: Quantum-dot dynamics under the amplification of an ultra-short pulse. Shown is the dynamics at the input facet (left column) and at the output facet (right column). (a),(b) Electric field amplitude (red line) and corresponding cross-correlation signal (black line). The lower panels show the time-trace of $(\mathbf{c}),(\mathrm{d})$ the normalized quantum-dot ground-state polarization amplitude and (e),(f) their inversion in dependence of the detuning of the individual quantum-dot subgroups from the signal. $\Theta=1.15 \pi, J=20 J_{0}$. Modified from [KOL13].

the resonant subgroup, as could have been expected, but instead shows rather strong asymmetry due to the asymmetric quantum-dot carrier distribution and the additional frequency components added to the optical pulse along its path.

Figure $4.22 \mathrm{e}$, f depict the corresponding inversion of the individual subgroups. As we have seen before, the optical pulse drives the population inversion below transparency for near-resonant subgroups, again confirming the occurrence of (partial) Rabi oscillations within the device.

In order to support our interpretation of Rabi-oscillation induced pulse-shaping in the device, we now simulate the quantum-dot semiconductor optical amplifier within the rate-equation approach, i.e., with adiabatically eliminated polarization. 


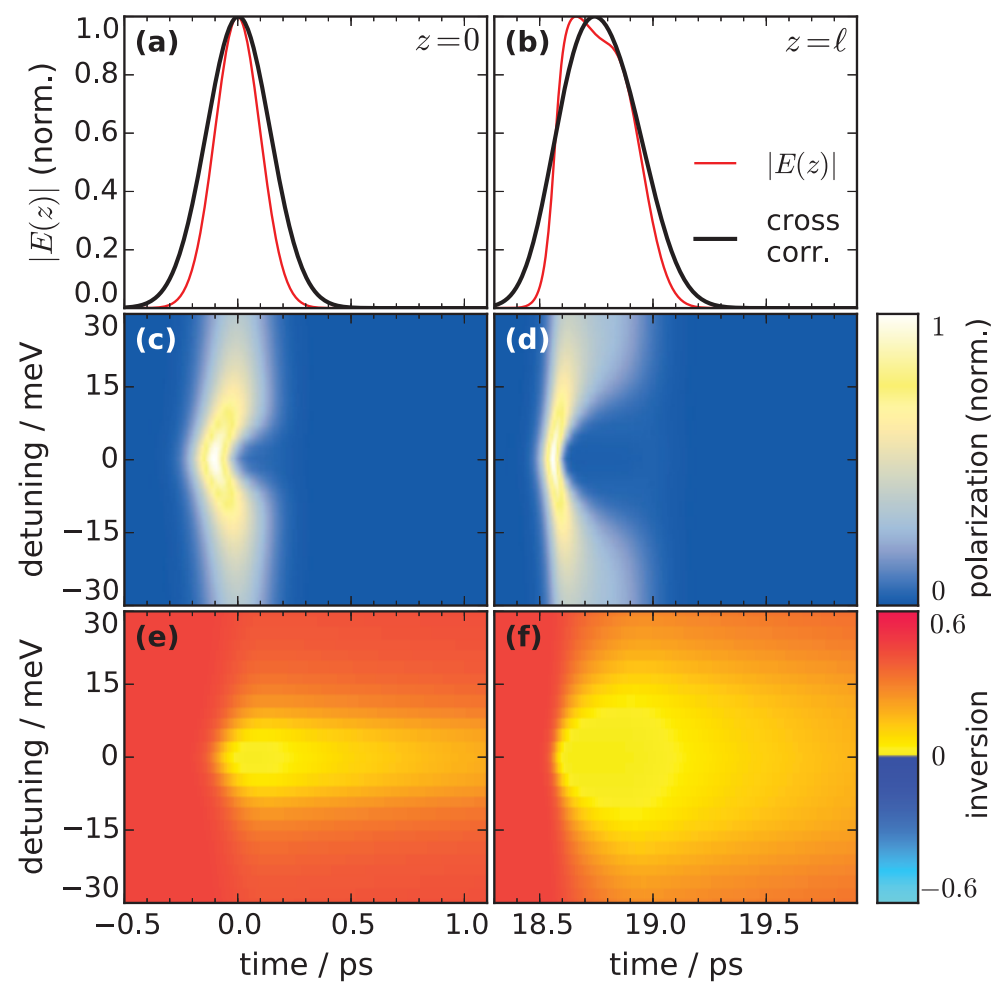

Figure 4.23.: Quantum-dot dynamics under the amplification of an ultra-short pulse using adiabatically eliminated microscopic polarization, cf. Fig. 4.22.

As we had derived earlier in Eq. (2.70), we can write for the polarization amplitudes in the limit of fast polarization decay

$$
p_{m}^{j}(t)=-i T_{2} \frac{\mu_{m} E(t)}{2 \hbar}\left(\rho_{e, m}^{j}(t)+\rho_{h, m}^{j}(t)-1\right)\left(\frac{1-i T_{2}\left(\omega_{m}^{j}-\omega\right)}{1+\left[T_{2}\left(\omega_{m}^{j}-\omega\right)\right]^{2}}\right) .
$$

We thus assume a quasi-static relation between the polarization and the incoming electric field. This, however, means that the dephasing process is treated as instantaneous, such that the polarization is assigned the value it would assume after complete dephasing. Subsequently, the rate-equation model cannot reproduce the pulse-shape modifications observed in the experiments, as demonstrated in Fig. 4.23.

Similar to the plots shown before in Fig. 4.22 we evaluate the inversion and polarization of each subgroup. The differences between the two approaches become immediately visible in the output pulse shape, which lacks the complex pulse shape we had seen before. While the front of the pulse shows a slight deformation, 
the cross-correlation amplitude reproduces a near-Gaussian shaped output pulse. This is due to the lack of autonomous polarization dynamics that was observed in Fig. $4.22 \mathrm{c}$,d. Here a quick buildup of the polarization amplitude can be seen, with a subsequent fast decay in the resonant subgroups. This decay is due to the "bleaching" of the corresponding subgroups (Fig. $4.22 \mathrm{e}, \mathrm{f}$ ). The inversion is depleted to near zero, rendering these subgroups transparent to the pulse. The rate-equation approach thus quite obviously cannot reproduce the observed dynamics.

In conclusion, we have demonstrated coherent interactions between ultra-short optical pulses and the quantum-dot active medium in quantum-dot semiconductor optical amplifiers. Provided strong enough input pulses and high enough time resolution, we predict the possibility to directly observe Rabi oscillations in pump-probe experiments. While the pronounced modifications to the electric field amplitudes due to the Rabi oscillations cannot be measured directly, we found that crosscorrelation measurements provide a reliable tool to identify Rabi oscillations by characteristic modifications of the cross-correlation envelope. By comparing corresponding experimental results with the predictions of our simulations, we have confirmed the occurrence of Rabi-oscillations in the quantum-dot active medium even at room temperature. This opens up the possibility for further quantumtheoretical research on the basis of macroscopic quantum-dot optical devices. 


\subsection{Conclusion}

In this chapter, we have studied the performance of semiconductor quantum-dot based optical amplifiers. A delay-differential equation model that incorporates microscopically calculated nonlinear charge-carrier scattering rates was presented, which can efficiently and accurately model the electric-field propagation through the amplifier device. The light-matter interaction is modeled on the basis of Maxwell-Bloch equations, taking the microscopic polarization of the quantum-dot medium into account. This approach yields the possibility to correctly describe the spectral properties of the spontaneous emission noise, as demonstrated by a comparison with experimentally obtained amplified spontaneous emission spectra.

We have performed studies of the large-signal amplification capabilities of the quantum-dot amplifier for optical signals that are resonant to the ground-state or excited-state transitions. A characterization of the device in terms of amplified spontaneous emission noise spectra has been presented, which shows very good agreement with experimental measurements on a corresponding quantumdot amplifier device. We have calculated its response to large-signal amplification in terms of gain-saturation characteristics and quality factor for the amplification of non-return-to-zero on-off-keyed pseudo-random bit sequences. Our results predict error-free (bit-error rate $<10^{-9}$ ) amplification of optical signals centered on the ground-state for input power ranges of $-25 \mathrm{dBm}<P_{\text {in }}^{\mathrm{GS}}<0 \mathrm{dBm}$. The signal quality was found to be limited by amplified spontaneous emission noise at lower input powers, and the onset of patterning effects due to the nonlinear amplification at higher powers.

The response of the quantum-dot amplifier on the excited state was found to be characterized by a higher gain but a stronger sensitivity to patterning effects, due to the onset of the nonlinear amplification regime for much lower input powers than on the ground state. This can be understood by the close coupling of the excited state to the charge-carrier reservoir, which leads to a stronger perturbation of the excited-state gain by variations of the total charge-carrier density. This results in a lower output saturation power of the excited state, and error-free amplification only for signals with input powers below $-15 \mathrm{dBm}$ at high pump currents.

We have subsequently investigated the possibility of a multi-state operation of the quantum-dot semiconductor optical amplifier. Here, two signals centered on the ground and excited state are being simultaneously amplified in a counterpropagating setup. We have performed similar studies of the large-signal gain and signal quality as before, now in dependence on the optical input powers on the ground and excited state and for different pump currents. Our model predicts a minor impact of an additional excited-state signal on the performance of the ground-state. For an input power of $0 \mathrm{dBm}$ on the excited state, we found a de- 
crease of the ground-state gain of only $5 \mathrm{~dB}$ compared to the solitary ground-state signal amplification. The signal quality on the ground state was found to decrease with increasing excited-state power, nevertheless staying well above a bit-error-rate of $10^{-9}$ for the considered input powers on the excited state below $0 \mathrm{dBm}$.

As before, the excited-state response has been demonstrated to be more sensitive to the presence of a signal on the ground-state. A strong decrease of both the largesignal gain and the quality-factor can be observed at low pump currents. Increasing the pump current can partly improve the excited-state performance, albeit under a decrease of the ground-state gain. Taking these results into account, we predict that the dual-state operation of the quantum-dot amplifier is possible for a intermediate pump current of $700 \mathrm{~mA}$ for input powers below $-7 \mathrm{dBm}$ on the ground-state and below $-13 \mathrm{dBm}$ on the excited-state, thus ensuring error-free amplification of the input signals and providing reasonable gain.

Furthermore, we have investigated the possibility of coherent pulse-shaping applications in quantum-dot semiconductor optical amplifiers. We have thus performed simulations of the propagation of ultra-short $(\approx 200 \mathrm{fs})$ optical pulses along the amplifier structure. Our model predicts the appearance of Rabi oscillations in the optical pulse and the quantum-dot active medium, which leads to a characteristic pulse shape modification of the output pulse. Depending on the input pulse area, a varying number of Rabi cycles is excited which becomes visible also in the integrated small-signal gain of the device.

Our theoretical results were applied in a comparison with experimental measurements performed in a cross-correlation setup. In the experiment, ultra-short optical pulses were amplified in a quantum-dot semiconductor optical amplifier. For different pump currents the cross-correlation between the amplified probe pulse and the reference input pulse was measured, showing clear pulse shape modifications for sufficiently high input powers. By calculating the corresponding signals from the theoretically obtained output pulses, we were able to directly compare the experiments with our predictions. The model results were found to match the experimental measurements very well. We could thus confirm that the changes in pulse shape are caused by Rabi oscillations, whereas an adiabatically eliminated microscopic polarization, i.e., an incoherent interaction, cannot reproduce the observed dynamics. Our results thus clearly show that macroscopic quantum-dot semiconductor optical devices are promising candidates for the applications of quantum-coherent effects. 
In this thesis, the dynamics of semiconductor quantum-dot optoelectronic devices has been investigated. The complex charge-carrier dynamics due to the coupling of localized electronic quantum-dot states and the surrounding continuum reservoir states has been found to crucially influence the dynamic response of the quantumdot devices to external perturbations.

A microscopically based balance equation model for semiconductor quantumdot lasers has been derived, which explicitly takes into account the microscopic Auger-scattering processes between the localized quantum-dot states and the surrounding charge-carrier reservoir. The resulting Boltzmann-like scattering terms ensure a realistic description of the quantum-dot charge-carrier dynamics and enables the possibility to describe intra-band nonequilibrium situations. Energy balance equations have been derived which allow the dynamic calculation of the charge-carrier quasi-equilibrium temperature, taking into account carrier-heating due to Auger-scattering. Together with a spectrally resolved inhomogeneously broadened quantum-dot ensemble, as well as a consistent description of the chargecarrier-induced gain and refractive index, the model is an adequate tool to describe quantum-dot laser dynamics.

The quantum-dot laser model was used to investigate its relaxation oscillations, which describe the intrinsic response of the laser device to perturbations from its steady-state. In dependence of the charge-carrier scattering rates, three qualitative different dynamic regimes have been found:

(i) For slow charge-carrier scattering, the reservoir states provide a nearly constant carrier-influx into the quantum-dots, leading to pronounced relaxation oscillations. This regime is called the "constant-reservoir regime", which is characterized by a strong nonequilibrium between the quantum-dot and reservoir states.

(ii) For intermediate scattering rates, in the "overdamped regime", the relaxation oscillation damping is strongly increased, leading to overdamped oscillations.

(iii) For fast scattering rates, the coupling between quantum-dot and reservoir states is strong enough to influence the reservoir carrier density. In this "synchronized regime", the reservoir carriers are thus closely coupled to the light- 
matter interaction in the quantum-dot states, leading to the reappearance of pronounced relaxation oscillations.

The reappearance of pronounced relaxation oscillations for high scattering was found to be caused by the detailed balance between charge-carrier capture and escape rates in the quantum-dot states. Quantum-dot laser models that do not take into account a dependence of the escape rates on the reservoir carrier density can therefore not describe the "synchronized regime". A minimal three-variable rate-equation model has been derived that takes this important effect into account. Using effective parameters, this minimal model has been shown to qualitatively reproduce the dynamics of the full balance equation model. This opens up the possibility of further analytical studies on quantum-dot laser dynamics using this simple, but sufficiently complex quantum-dot laser model.

The dynamic regimes were found to crucially influence the response of the quantum-dot laser to external modulations and perturbations. While in the synchronized regime the modulation bandwidth is well described by the conventional analytical expression that take the relaxation oscillation damping and frequency into account, the actual modulation response differs from this prediction in the overdamped and constant-reservoir regimes.

The amplitude-phase coupling in semiconductor lasers, commonly described by an $\alpha$-factor, was found to fail in describing the quantum-dot laser dynamics. This has been demonstrated for quantum-dot lasers under optical injection and timedelayed optical feedback. While fixed points and their bifurcations are well described by an $\alpha$-factor, it cannot be used to describe time-varying solutions. This is due to a frequency-dependent amplitude-phase coupling, leading to different frequency-responses at different perturbation frequencies. An $\alpha$-factor cannot describe this behavior and generally overestimates the extent of complex laser dynamics. This discrepancy was found to be especially pronounced when the laser operates within the constant-reservoir regime, due to the strong nonequilibrium between the resonant and off-resonant electronic states.

The investigations in this work have thus shown that many conventional frameworks for the description of semiconductor laser dynamics fail when applied to quantum-dot lasers. This discrepancy is due to the possible intra-band nonequilibrium situations between the charge-carrier states. In order to correctly model and describe the quantum-dot laser dynamics, an accurate and consistent description of the complex charge-carrier dynamics and charge-carrier induced gain and refractive index is required. Three quantum-dot laser models on different levels of sophistication which fulfill this requirement have been derived and presented, which can be used in future works for an accurate representation of quantum-dot lasers. Specifically, a simple three-variable rate-equation model has been developed that 
correctly predicts the three dynamic regimes in dependence of the scattering rate. This model could be used in future studies for a deeper analytical understanding of quantum-dot lasers. A ten-variable rate-equation model has been successfully implemented in path-continuation routines for the investigation of the occurring bifurcations in an optical injection setup. This model could be extended, e.g., to optical feedback, and can be used in further bifurcation studies.

In the second part of this thesis, two types of experimental applications unique to quantum-dot semiconductor amplifiers have been investigated. To this end, the quantum-dot laser balance equation model was extended to a delay-differentialequation model, that explicitly takes into account the electric field propagation along the device.

It was found that quantum-dot semiconductor optical amplifiers enable ultrabroad-band amplification of optical data signals by utilizing the localized ground and excited-state transitions. The performance of a quantum-dot amplifier under large-signal amplification has been analyzed in terms of the gain and quality factor of an optical data signal, centered on either of the first two localized quantumdot states. While the excited-state generally shows poorer performance than the ground-state, the results indicate that an effective application of the amplifier on both wavelength ranges is possible. Furthermore, the dual-state operation under simultaneous amplification of data signals on the two wavelengths in a counterpropagating setup was investigated. The theoretical results predict a parameter range for which an effective amplification of both data streams, in terms of gain and signal quality, is possible. The unique electronic density of states and the efficient charge-carrier refilling by the reservoir could thus be used to simplify optical data communication networks by using a single quantum-dot semiconductor optical amplifier for the simultaneous amplification of different wavelength bands.

Furthermore, it was shown that quantum-dot semiconductor optical amplifiers exhibit coherent effects on the macroscopic scale. Experimental results on the pulse-shaping of ultra-short optical pulses propagating through a quantum-dot amplifier at room temperature have been compared with the theoretical predictions. The observed pulse-shape modifications have been unambiguously explained by the occurrence of Rabi oscillations in the quantum-dot active medium. Macroscopic quantum-dot devices have thus been shown to exhibit coherent pulse-shaping effects, owing to the long dephasing time of the localized quantum-dot states compared to conventional semiconductor active materials. The application of semiconductor quantum-dot active media might therefore open up the possibility of novel quantum-optical devices. 


\section{A.1 Evaluation of RO Parameters from Numerical Time- Series}

In the numerical evaluation of the relaxation oscillation parameters the time-series of the output power is fitted in order to extract the corresponding eigenvalues. We chose an exponentially damped harmonic oscillation with an underlying slow exponential decay in order to fit the numerical results:

$$
S^{\mathrm{fit}}(t)=S_{0}\left[1+a \cos \left(\omega_{\mathrm{RO}} t-\varphi_{0}\right) e^{-\Gamma_{\mathrm{RO}} t}+b e^{-\gamma t}\right] .
$$

The fitting parameters are the steady-state power $S_{0}$, the RO amplitude $a$, the RO frequency $\omega_{\mathrm{RO}}$, the phase shift $\varphi_{0}$, the RO damping $\Gamma_{\mathrm{RO}}$, the amplitude of the additional exponential decay $b$, and the corresponding decay rate $\gamma$. This additional decay term describes the (often slow) relaxation of the reservoir chargecarriers, and is related to the third eigenvalue $\lambda_{3}$ found in the minimal QD laser model (see Eq. (3.35)).

\section{A.2 Analytic Approximation of RO Parameters in the Synchronized Regime}

The zeroth-order approximations in the limit $R \rightarrow \infty$ of the three-variable minimal quantum-dot laser model (Eqs. (3.27)) are given by

$$
\begin{aligned}
\Gamma_{\mathrm{RO}}^{\mathrm{s}, 0} & =\frac{1}{2(1+d)}\left(\frac{1}{T_{1}}+\frac{d}{T_{\mathrm{sp}}}+2 d g S^{*}\right), \\
\omega_{\mathrm{RO}}^{\mathrm{s}, 0} & =\sqrt{4 d g \kappa S^{*}-\Gamma_{\mathrm{RO}}^{\mathrm{s}, 0^{2}}},
\end{aligned}
$$

(see Eqs. (3.42)). The eigenvalue is expanded as

$$
\lambda=\lambda_{0}+\frac{1}{R} \lambda_{1}+O\left(\frac{1}{R^{2}}\right) .
$$


We insert the above equations into the characteristic equation Eq. (3.30) and take into account only the terms of order $O(1)$ with respect to $R$. As an additional simplification, we take the limit $T_{1}, T_{\mathrm{sp}} \rightarrow \infty$, i.e., neglect effects due to the chargecarrier lifetimes in the first-order correction, as the scattering rate $R$ is much greater than the respective decay rates. The reduced characteristic equation is given by

$$
0=2 g S^{*}\left(2 \kappa \lambda_{0}+\lambda_{0}^{2}+d \lambda_{1}\right)+\lambda_{0}\left(\lambda_{0}^{2}+2(1+d) \lambda_{1}\right)+O\left(\frac{1}{R}\right)
$$

This yields for the first order correction in $\frac{1}{R}$ :

$$
\lambda_{1}=\frac{\lambda_{0}\left(\lambda_{0}^{2}+2 g S^{*}\left(2 \kappa+\lambda_{0}\right)\right)}{2(1+d) \lambda_{0}+2 d g S^{*}} .
$$

This expression can be expanded in terms of real and imaginary part, giving the first-order corrections in the relaxation oscillation damping and frequency, respectively:

$$
\begin{aligned}
& \Gamma_{\mathrm{RO}}^{\mathrm{s}, 1}=\frac{1}{2(1+d) T_{1} T_{\mathrm{sp}}}[ 1+3\left(\Gamma_{\mathrm{RO}}^{\mathrm{s}, 0}\right)^{2} T_{1} T_{\mathrm{sp}}+2 g S\left(1+2 k T_{1}\right) T_{\mathrm{sp}} \\
&-\left.2 \Gamma_{\mathrm{RO}}^{\mathrm{s}, 0}\left(T_{1}+T_{\mathrm{sp}}+2 g S T_{1} T_{\mathrm{sp}}\right)-T_{1} T_{\mathrm{sp}}\left(\omega_{\mathrm{RO}}^{\mathrm{s}, 0}\right)^{2}\right], \\
& \omega_{\mathrm{RO}}^{\mathrm{s}, 1}=-\frac{1}{2(1+d) T_{1} T_{\mathrm{sp}} \omega_{\mathrm{RO}}^{\mathrm{s}, 0}}\left[\left(\Gamma_{\mathrm{RO}}^{\mathrm{s}, 0}\right)^{3} T_{1} T_{\mathrm{sp}}-\left(\Gamma_{\mathrm{RO}}^{\mathrm{s}, 0}\right)^{2}\left(T_{1}+T_{\mathrm{sp}}+2 g S T_{1} T_{\mathrm{sp}}\right)\right. \\
&+\left(T_{1}+T_{\mathrm{sp}}\right)\left(\omega_{\mathrm{RO}}^{\mathrm{s}, 0}\right)^{2}+2 g S T_{\mathrm{sp}}\left(-2 k+T_{1}\left(\omega_{\mathrm{RO}}^{\mathrm{s}, 0}\right)^{2}\right) \\
&\left.+\Gamma_{\mathrm{RO}}^{\mathrm{s}, 0}\left(1+2 g S\left(1+2 k T_{1}\right) T_{\mathrm{sp}}-3 T_{1} T_{\mathrm{sp}}\left(\omega_{\mathrm{RO}}^{\mathrm{s}, 0}\right)^{2}\right)\right] .
\end{aligned}
$$




\section{LIST OF FIGURES}

1.1. Sketch of the density of states depending on the dimensionality. . . . 1

1.2. Sketch of different approaches to charge-carrier confinement in semiconductor heterostructures. . . . . . . . . . . . . . 3

1.3. Sketch of the epitaxial Stranski-Krastanov growth of a DWELL

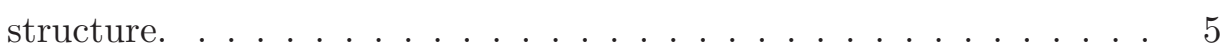

1.4. Sketch of the energy structure of a DWELL structure. . . . . . . . 5

2.1. Scattering mechanisms in semiconductor structures. . . . . . . . . 11

2.2. Illustration of carrier heating by Auger-scattering. . . . . . . . . . . 12

2.3. Possible scattering channels in QD-QW systems. . . . . . . . . . 16

2.4. Illustration of the geometric confinement factor. . . . . . . . . . . . 25

2.5. Energy scheme of the considered dot-in-a-well (DWELL) structure. . 31

2.6. Illustration of the quantum-dot subgroups to model inhomogeneous broadening. . . . . . . . . . . . . . . . 33

2.7. Lorentzian gain profile of a single optical transition. . . . . . . . . . 37

2.8. Mechanism of Auger-heating in QD devices. . . . . . . . . . . . . . 45

3.1. Relaxation oscillations in a two-variable laser rate-equation system. . 52

3.2. Energy scheme of the localized quantum-dot states. . . . . . . . . . 54

3.3. Simulated QD laser light-current characteristics. . . . . . . . . . . 56

3.4. Simulated QD laser turn-on dynamics. . . . . . . . . . . . . . 57

3.5. Simulated QD laser turn-on dynamics for different scattering rates. . 59

3.6. Relaxation oscillation parameters in dependence of the scattering rates. . . . . . . . . . . . . . . . . . . . 61 61

3.7. Eigenvalues and relaxation oscillation parameters in the minimal QD model. . . . . . . . . . . . . . . . . . . . 65

3.8. Analytic approximation for RO parameters in the constant-reservoir regime. . . . . . . . . . . . . . . . 69 
3.9. Analytic approximation for RO parameters in the synchronized regime. 71

3.10. Small-signal modulation transfer function for the two-variable rateequation system. . . . . . . . . . . . . . . . 74

3.11. Small-signal modulation response of the QD laser. . . . . . . . . 75

3.12. Small-signal modulation bandwidth of the QD laser. . . . . . . . 76

3.13. Scattering-rate dependence of the QD laser modulation response. . . 78

3.14. Sketch of the $\alpha$-factor in conventional laser models. . . . . . . . . . . 82

3.15. Reaction of the QD laser to optical and pump-current perturbations. 85

3.16. Charge-carrier densities of the QD laser under optical and pumpcurrent perturbations. . . . . . . . . . . . 86

3.17. Sketch of the optical injection scheme. . . . . . . . . . . . 88

3.18. Optical injection into the laser cavity. . . . . . . . . . . . . 89

3.19. Numerically simulated bifurcation diagram of the quantum-dot laser under optical injection. . . . . . . . . . . . . . . . . . 92

3.20. Saddle-node-infinite-period bifurcation. . . . . . . . . . . . . . . 93

3.21. Phase-unbounding of the optically injected quantum-dot laser after a Hopf bifurcation. . . . . . . . . . . . . . . 95

3.22. Period-doubling bifurcations to chaos. . . . . . . . . . . 96

3.23. Additional maxima due to deformation of the periodic orbit. . . . . 97

3.24. Energy scheme of the localized quantum-dot states for the deep-dot structure. . . . . . . . . . . . . . . . . 98

3.25. Numerically simulated bifurcation diagram of the shallow-dot quantumdot laser under optical injection for different pump currents. . . . . . 99

3.26. Numerically simulated bifurcation diagram of the deep-dot quantumdot laser under optical injection for different pump currents. . . . . . 100

3.27. Analytical SNIPER bifurcation line. . . . . . . . . . . . . . . 102

3.28. Comparison of the injection-locking range under optical injection between the full model and the $\alpha$-factor approach (shallow-dot, $J=$

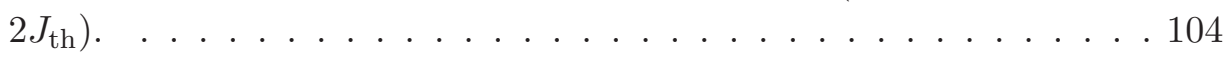

3.29. Comparison of the bifurcation diagram under optical injection between the full model and the $\alpha$-factor approach (shallow-dot, $J=$

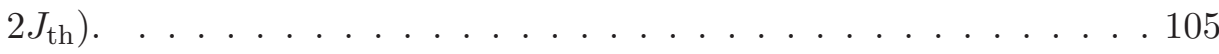

3.30. Comparison of the bifurcation diagram under optical injection between the full model and the $\alpha$-factor approach (shallow-dot, $J=$

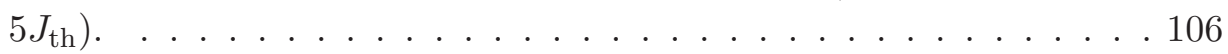

3.31. Transients of the complex gain in the optically injected shallow-dot laser. . . . . . . . . . . . . . . . . . 107 
3.32. Evaluation of the amplitude-phase coupling in the optically injected shallow-dot laser. . . . . . . . . . . . . . . . . . . . . . 109

3.33. Comparison of the bifurcation diagram under optical injection between the full model and the $\alpha$-factor approach (deep-dot, $J=5 J_{\mathrm{th}}$ ). 111

3.34. Evaluation of the amplitude-phase coupling in the optically injected deep-dot laser. . . . . . . . . . . . . . . . . . . 112

3.35. Fits of the scattering rates for the shallow-dot device. . . . . . . . 118

3.36. Comparison of the full model and the simplified 10-variable rate equation system. . . . . . . . . . . . . . . . . . . . . 119

3.37. Path-continuation results for the optically injected shallow-dot laser. 121

3.38. Comparison between $\alpha$ and the full gain dynamics in the simplified quantum-dot model (path-continuation) . . . . . . . . . . . . 122

3.39. Comparison between $\alpha$ and the full gain dynamics for the optically injected shallow-dot laser with reduced scattering rates (pathcontinuation, simplified model) . . . . . . . . . . . . . . 123

3.40. Comparison between $\alpha$ and the full gain dynamics for the optically injected shallow-dot laser with reduced scattering rates and reduced losses (path-continuation, simplified model). . . . . . . . . . . . . 124

3.41. Sketch of the optical time-delayed feedback scheme. . . . . . . . . 125

3.42. Numerically simulated bifurcation diagram of the shallow-dot laser in the feedback strength under optical feedback. . . . . . . . . . 128

3.43. Numerically simulated bifurcation diagram of the shallow-dot laser under optical feedback. . . . . . . . . . . . . . . . . 129

3.44. Numerically simulated bifurcation diagram of the deep-dot laser under optical feedback. . . . . . . . . . . . . . . . 130

3.45. Numerically simulated bifurcation diagram of the shallow-dot laser in the feedback strength under optical feedback. . . . . . . . . . . . 131

3.46. Comparison of the full model and $\alpha$-factor approach of the shallowdot laser dynamics under optical feedback. . . . . . . . . . . . . 132

3.47. Comparison of the full model and $\alpha$-factor approach of the deep-dot laser dynamics under optical feedback. . . . . . . . . . . . . 133

3.48. Small-signal modulation response of the quantum-dot laser under current and electric field modulation. . . . . . . . . . . . 136

3.49. Small-signal modulation response of the quantum-dot laser under current and electric field modulation for reduced scattering rates and reservoir losses. . . . . . . . . . . . . . . . . 138 
3.50. Dependence of the frequency-modulation response on the effective scattering rate. . . . . . . . . . . . . . . . . . . 140

4.1. Sketch of the quantum-dot semiconductor optical amplifier. . . . . . 147

4.2. Space discretization scheme of the QDSOA device with length $\ell$ used in the DDE model. . . . . . . . . . . . . . . . . . . . . 149

4.3. Comparison of simulated and measured pump-current dependent ASE spectra. . . . . . . . . . . . . . . . . . . 155

4.4. Fitted pump-current dependence of the QDSOA model parameters. . 157

4.5. Comparison between simulations and experiments of the optical GS and ES gain depending on the pump current. . . . . . . . . . 158

4.6. Gain saturation of the QDSOA under amplification of cw signals centered on the GS or ES frequency. . . . . . . . . . . . 160

4.7. Pump-current dependence of the saturation input and output power of ground and excited state. . . . . . . . . . . . . . 161

4.8. Illustration of the non-return-to-zero pulse amplitude modulation scheme. . . . . . . . . . . . . . . . . 161

4.9. Evaluation of the quality factor from optical eye diagrams. . . . . . . 162

4.10. Input-power dependence of the quality-factor for optical bit patterns at different pump currents. . . . . . . . . . . . . 163

4.11. Eye diagrams for different optical input power on the ground-state. . 164

4.12. Sketch of the multi-state operation of the quantum-dot semiconductor optical amplifier. . . . . . . . . . . . . . . 165

4.13. Gain dependence on GS and ES input powers in multi-state operation of the QDSOA. . . . . . . . . . . . . 166

4.14. GS quality-factor dependence on GS and ES input powers in multistate operation of the QDSOA. . . . . . . . . . . 167

4.15. Ground-state eye diagrams under perturbation by an excited-state signal. . . . . . . . . . . . . . . . . . 168

4.16. Excited-state eye diagrams under perturbation by a ground-state signal. . . . . . . . . . . . . . . . . . . 169

4.17. Sketch of the Rabi-oscillation induced pulse shaping in QDSOAs. . . 171

4.18. Fitted dephasing times for the QDSOA devices in Sections 4.3 and

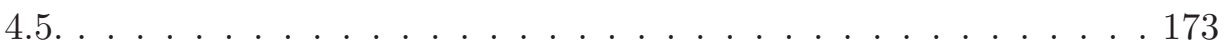

4.19. Rabi-oscillations in the quantum-dot semiconductor optical amplifier. 175 4.20. Simplified sketch of the heterodyne measurement technique. . . . . . 177 
4.21. Comparison of the Rabi-oscillations in the amplified ultra-short pulses between experiment and theory. . . . . . . . . . . . 178

4.22. Quantum-dot polarization dynamics under the amplification of an ultra-shot pulse. . . . . . . . . . . . . . . 180

4.23. Quantum-dot dynamics under the amplification of an ultra-shot pulse in the rate-equation limit. . . . . . . . . . . . . . . 181 


\section{LIST OF TABLES}

3.1. Parameters used in the quantum-dot laser balance equation model. . 55

3.2. Parameters used in the minimal quantum-dot laser model. . . . . . . 65

3.3. Fitting parameters for the shallow-dot scattering rates. . . . . . . . 117

3.4. Parameters used in the simplified 10 -variable rate-equation model for the shallow-dot laser. . . . . . . . . . . . . . . . . . . . 120

4.1. Model parameters used in the QDSOA simulations in sections 4.3

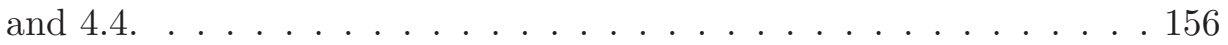

4.2. Model parameters used in the QDSOA simulations in section 4.5. . . 173

A.1. List of commonly used symbols. . . . . . . . . . . . . . . . . . . 199

A.2. List of commonly used acronyms. . . . . . . . . . . . . . . . 200 
Table A.1.: List of commonly used symbols.

\begin{tabular}{|c|c|}
\hline SYMBOL & MEANING \\
\hline$-e_{0}$ & Electron charge. \\
\hline$c_{0}$ & Vacuum speed of light. \\
\hline$\varepsilon_{0}, \varepsilon_{\mathrm{bg}}$ & Vacuum and background dielectric constants. \\
\hline$N^{\mathrm{QD}}$ & QD sheet density per QW layer. \\
\hline$a_{L}$ & Number of QW layers. \\
\hline$\rho_{b, m}^{j}$ & QD occupation of the $j^{\text {th }}$ subgroup in the $m^{\text {th }}$ state $(b \in\{e, h\})$. \\
\hline$p_{m}^{j}$ & QD microscopic polarization of the $j^{\text {th }}$ subgroup in the $m^{\text {th }}$ state. \\
\hline$T_{2}^{m}$ & Dephasing time of the microscopic polarization. \\
\hline$\omega_{m}^{j}$ & QD subgroup transition frequencies. \\
\hline$\mu_{m}$ & Optical transition dipole moment in the $m^{\text {th }}$ QD state. \\
\hline$g_{m}^{j}$ & QD gain coefficients under adiabatically eliminated polarization. \\
\hline$\nu_{m}$ & Degree of degeneracy of the $m^{\text {th }}$ QD state, excluding spin. \\
\hline$f^{j}$ & Probability mass function of the $j^{\text {th }}$ QD subgroup. \\
\hline$W_{m}$ & Spontaneous recombination rate in the $m^{\text {th }}$ QD state. \\
\hline$\beta$ & Spontaneous emission ratio. \\
\hline$\Gamma$ & Geometric confinement factor. \\
\hline$\omega$ & Frequency of the reference frame, $\approx$ electric field carrier-frequency. \\
\hline$w_{b}$ & QW charge-carrier density. \\
\hline$A^{S}$ & Linear recombination rate in the carrier-reservoir. \\
\hline$B^{S}$ & Bimolecular recombination rate in the carrier-reservoir. \\
\hline$A_{\text {act }}$ & In-plane active-region area. \\
\hline$\Delta E_{b}$ & Energy separation between QD GS and QW band edge. \\
\hline$\Delta_{b}$ & Energy separation between QD GS and QD ES. \\
\hline$\Delta E_{\mathrm{inh}}$ & QD inhomogeneous broadening width (FWHM). \\
\hline$S_{b, m}^{\mathrm{cap}, \text { in }}, S_{b}^{\mathrm{rel}, \text { in }}$ & QD direct-capture and relaxation in-scattering rates. \\
\hline$\Delta \nu_{\text {inj }}$ & Frequency detuning of the optically injected master signal from the slave laser. \\
\hline$K$ & Injection strength, relative to the optical loss rate $\kappa$. \\
\hline$\tau$ & Optical feedback time delay. \\
\hline$C$ & Feedback phase shift. \\
\hline$K_{\mathrm{fb}}$ & Feedback strength, relative to the optical loss rate $\kappa$. \\
\hline$B E R$ & Optical data signal bit-error-rate. \\
\hline$Q$ & Optical data signal quality factor. \\
\hline$\Theta$ & Optical input pulse area. \\
\hline
\end{tabular}


TABLE OF SYMBOLS

Table A.2.: List of commonly used acronyms.

\begin{tabular}{cl}
\hline \hline ACRONYM & MEANING \\
\hline CW & constant-wave \\
QD & quantum-dot \\
QW & quantum-well \\
DWELL & dot-in-a-well \\
RO & relaxation oscillation \\
SNIPER & saddle-node-infinite-period (bifurcation) \\
QDSOA & Quantum-dot semiconductor optical amplifier \\
\hline \hline
\end{tabular}




\section{List of PUBLICATIONS}

Parts of this work have been previously published in:

[LIN12] B. Lingnau, K. Lüdge, W. W. Chow, and E. Schöll: Influencing modulation properties of quantum-dot semiconductor lasers by electron lifetime engineering, Appl. Phys. Lett. 101, 131107 (2012).

[LIN12b] B. Lingnau, K. Lüdge, W. W. Chow, and E. Schöll: Failure of the $\alpha$ factor in describing dynamical instabilities and chaos in quantum-dot lasers, Phys. Rev. E 86, 065201(R) (2012).

[WIL12c] S. Wilkinson, B. Lingnau, J. Korn, E. Schöll, and K. Lüdge: Influence of noise on the signal properties of quantum-dot semiconductor optical amplifiers, IEEE J. Sel. Top. Quantum Electron. 19, 1900106 (2013).

[LIN13] B. Lingnau, W. W. Chow, E. Schöll, and K. Lüdge: Feedback and injection locking instabilities in quantum-dot lasers: a microscopically based bifurcation analysis, New J. Phys. 15, 093031 (2013).

[KOL13] M. Kolarczik, N. Owschimikow, J. Korn, B. Lingnau, Y. Kaptan, D. Bimberg, E. Schöll, K. Lüdge, and U. Woggon: Quantum coherence induces pulse shape modification in a semiconductor optical amplifier at room temperature, Nat. Commun. 4, 2953 (2013).

[LIN14] B. Lingnau, W. W. Chow, and K. Lüdge: Amplitude-phase coupling and chirp in quantum-dot lasers: influence of charge carrier scattering dynamics, Opt. Express 22, 4867-4879 (2014).

[SCH15] H. Schmeckebier, B. Lingnau, S. König, K. Lüdge, C. Meuer, A. Zeghuzi, D. Arsenijević, M. Stubenrauch, R. Bonk, C. Koos, C. Schubert, T. Pfeiffer, and D. Bimberg: Ultra-broadband bidirectional dual-band quantum-dot semiconductor optical amplifier, Optical Fiber Communication Conference and Exposition accepted (2015). 


\section{LIST OF PUBLICATIONS}

The research that lead to this thesis has also lead to the following collaborations:

[LIN12t] B. Lingnau, K. Lüdge, W. W. Chow, and E. Schöll: Many-body effects and self-contained phase dynamics in an optically injected quantum-dot laser, SPIE Proc. 8432 (2012).

[ZIE13] D. Ziemann, R. Aust, B. Lingnau, E. Schöll, and K. Lüdge: Optical injection enables coherence resonance in quantum-dot lasers, Europhys. Lett. 103, 14002-p1-14002-p6 (2013).

[SCH13g] F. Schulze, B. Lingnau, S. M. Hein, A. Carmele, E. Schöll, K. Lüdge, and A. Knorr: Feedback-induced steady-state light bunching above the lasing threshold, Phys. Rev. A 89, 041801(R) (2014).

[LIN14p] B. Lingnau and K. Lüdge: Quantenpunktlaser - Laserlicht auf den Punkt gebracht, Physik unserer Zeit 45, 140-146 (2014).

[KAP14b] Y. Kaptan, A. Röhm, B. Herzog, B. Lingnau, H. Schmeckebier, D. Arsenijević, V. Mikhelashvili, O. Schops, M. Kolarczik, G. Eisenstein, D. Bimberg, U. Woggon, N. Owschimikow, and K. Lüdge: Stability of a quantum dot excited state laser during simultaneous ground state amplification, Appl. Phys. Lett. 105, 191105-1-191105-4 (2014).

[WAN14b] C. Wang, B. Lingnau, K. Lüdge, J. Even, and F. Grillot: Enhanced dynamic performance of quantum dot semiconductor lasers operating on the excited state, IEEE J. Quantum Electron. 50, 723-731 (2014).

[GRO14] N. B. Grosse, N. Owschimikow, R. Aust, B. Lingnau, A. Koltchanov, M. Kolarczik, K. Lüdge, and U. Woggon: Pump-probe quantum state tomography in a semiconductor optical amplifier, Opt. Express 22, $32520-32525$ (2014).

[ROE15a] A. Röhm, B. Lingnau, and K. Lüdge: Understanding ground-state quenching in quantum-dot lasers, IEEE J. Quantum Electron. $\mathbf{5 1}$ (2015). 


\section{BIBLIOGRAPHY}

[ABA01] H. D. I. Abarbanel, M. B. Kennel, L. Illing, S. Tang, H. F. Chen, and J. M. Liu: Synchronization and communication using semiconductor lasers with optoelectronic feedback, IEEE J. Quantum Electron. 37, 1301-1311 (2001).

[ACH06a] M. Achermann, A. P. Bartko, J. A. Hollingsworth, and V. I. Klimov: The effect of auger heating on intraband carrier relaxation in semiconductor quantum rods, Nature Physics 2, 557-561 (2006).

[ADA85a] M. J. Adams, J. V. Collins, and I. D. Henning: Analysis of semiconductor laser optical amplifiers, IEE Proc. Optoelectron. 132, 58-63 (1985).

[ADL73] R. Adler: A study of locking phenomena in oscillators, Proc. IEEE 61, 13801385 (1973).

[AGH09] H. Aghajanpour, V. Ahmadi, and M. Razaghi: Ultra-short optical pulse shaping using semiconductor optical amplifier, Opt. \& Laser Tech. 41, 654-658 (2009).

[AGR93a] G. P. Agrawal and C. M. Bowden: Concept of linewidth enhancement factor in semiconductor lasers: its usefulness and limitations, IEEE Photonics Technol. Lett. 5, 640-642 (1993).

[AHL06a] A. Ahlborn and U. Parlitz: Laser stabilization with multiple-delay feedback control, Opt. Lett. 31, 465-467 (2006).

[AKI02] T. Akiyama, H. Kuwatsuka, N. Hatori, Y. Nakata, H. Ebe, and M. Sugawara: Symmetric highly efficient $(\sim 0 d B)$ wavelength conversion based on four-wave mixing in quantum dot optical amplifiers, IEEE Photon. Technol. Lett. 14, 11391141 (2002).

[AKI03] T. Akiyama, N. Hatori, Y. Nakata, H. Ebe, and M. Sugawara: Pattern-effectfree amplification and cross-gain modulation achieved by using ultrafast gain nonlinearity in quantum-dot semiconductor optical amplifiers, phys. stat. sol. (b) 238, 301-304 (2003).

[ALB11] F. Albert, C. Hopfmann, S. Reitzenstein, C. Schneider, S. Höfling, L. Worschech, M. Kamp, W. Kinzel, A. Forchel, and I. Kanter: Observing chaos for quantumdot microlasers with external feedback, Nature Communications 2, 366 (2011). 
[ALE07] R. R. Alexander, D. Childs, H. Agarwal, K. M. Groom, H. Y. Liu, M. Hopkinson, and R. A. Hogg: Zero and controllable linewidth enhancement factor in p-doped 1.3 $\mu \mathrm{m}$ quantum dot lasers, Jpn. J. Appl. Phys. 46, 2421 (2007).

[ALF68] Z. I. Alferov, V. M. Andreev, V. I. Korolkov, E. L. Portnoi, and D. N. Tretyakov: Coherent radiation of epitaxial heterojunction structures in the AlAs-GaAs system, Fiz. Tekh. Poluprovodn. 2, 1545-1547 (1968).

[ARA82] Y. Arakawa and H. Sakaki: Multidimensional quantum well laser and temperature dependence of its threshold current, Appl. Phys. Lett. 40, 939 (1982).

[ARE84] F. T. Arecchi, G. L. Lippi, G. P. Puccioni, and J. R. Tredicce: Deterministic chaos in laser with injected signal, Opt. Commun. 51, 308-315 (1984).

[ARN65] V. I. Arnold: Small denominators $i$, mappings of the circumference onto itself, Am. Math. Soc. Transl. 46, 213-284 (1965).

[ARS13] D. Arsenijević, M. Kleinert, and D. Bimberg: Phase noise and jitter reduction by optical feedback on passively mode-locked quantum-dot lasers, Appl. Phys. Lett. 103, 231101 (2013).

[ASA86] M. Asada, Y. Miyamoto, and Y. Suematsu: Gain and the threshold of threedimensional quantum-box lasers, IEEE J. Quantum Electron. 22, 1915-1921 (1986).

[ASA89] M. Asada: Intraband relaxation time in quantum-well lasers, IEEE J. Quantum Electron. 25, 2019-2026 (1989).

[ASR10] L. V. Asryan and R. A. Suris: Upper limit for the modulation bandwidth of a quantum dot laser, Appl. Phys. Lett. 96, 221112 (2010).

[BAN98] L. Bányai, Q. T. Vu, B. Mieck, and H. Haug: Ultrafast quantum kinetics of time-dependent RPA-screened coulomb scattering, Phys. Rev. Lett. 81, 882-885 (1998).

[BAV10] P. Baveja, D. Maywar, A. Kaplan, and G. P. Agrawal: Self-phase modulation in semiconductor optical amplifiers: Impact of amplified spontaneous emission, IEEE J. Quantum Electron. 46, 1396-1403 (2010).

[BEA59] A. Beattie and P. T. Landsberg: Auger effect in semiconductors, Proc. R. Soc. A 249, 16-29 (1959).

[BEN00a] O. Benson, C. Santori, M. Pelton, and Y. Yamamoto: Regulated and entangled photons from a single quantum dot, Phys. Rev. Lett. 84, 2513-2516 (2000).

[BER03b] T. W. Berg and J. Mørk: Quantum dot amplifiers with high output power and low noise, Appl. Phys. Lett. 82, 3083-3085 (2003).

[BER04a] T. W. Berg and J. Mørk: Saturation and noise properties of quantum-dot optical amplifiers, IEEE J. Quantum Electron. 40, 1527-1539 (2004). 
[BER04] T. W. Berg, J. Mørk, and J. M. Hvam: Gain dynamics and saturation in semiconductor quantum dot amplifiers, New J. Phys. 6, 178 (2004).

[BES93] P. Besnard, B. Meziane, and G. M. Stephan: Feedback phenomena in a semiconductor laser induced by distant reflectors, IEEE J. Quantum Electron. 29, 1271-1284 (1993).

[BIM97a] D. Bimberg, N. Kirstaedter, N. N. Ledentsov, Z. I. Alferov, P. S. Kop'ev, and V. Ustinov: InGaAs-GaAs quantum-dot lasers, IEEE J. Sel. Top. Quantum Electron. 3, 196-205 (1997).

[BIM97] D. Bimberg, N. N. Ledentsov, M. Grundmann, and F. Heinrichsdorff: Edge und Surface Emitting Quantum Dot Lasers, IEDM Tech. Dig., 381-384 (1997).

[BIM08] D. Bimberg: Quantum dot based nanophotonics and nanoelectronics, Electron. Lett. 44, 168 (2008).

[BIM08a] D. Bimberg: Semiconductor Nanostructures (Springer, Berlin, 2008).

[BIM12] D. Bimberg: Vom hässlichen Entlein zum Schwan-vor fünfzig Jahren wurde der Halbleiterlaser erfunden., Physik Journal Mai (2012).

[BIN92] K. Binder: The Monte Carlo Method in Condensed Matter Physics (Springer Verlag, Berlin, 1992).

[BIN92a] R. Binder, D. Scott, A. E. Paul, M. Lindberg, K. Henneberger, and S. W. Koch: Carrier-carrier scattering and optical dephasing in highly excited semiconductors, Phys. Rev. B 45, 1107 (1992).

[BOE15] F. Böhm, A. Zakharova, E. Schöll, and K. Lüdge: Amplitude-phase coupling drives chimera states in globally coupled laser networks, Phys. Rev. E 91, 040901 (R) (2015).

[BOL91] K. J. Boller, A. Imamoglu, and S. E. Harris: Observation of electromagnetically induced transparency, Phys. Rev. Lett. 66, 2593-2596 (1991).

[BON11] R. Bonk, T. Vallaitis, J. Guetlein, C. Meuer, H. Schmeckebier, D. Bimberg, C. Koos, and J. Leuthold: The input power dynamic range of a semiconductor optical amplifier and its relevance for access network applications, IEEE Photon. J. 3, 1039-1053 (2011).

[BOR97a] P. Borri, S. Ceccherini, M. Gurioli, and F. Bogani: Auger heating of carriers in GaAs/AlAs heterostructures, Solid State Commun. 103, 77-81 (1997).

[BOR00] P. Borri, W. Langbein, J. M. Hvam, F. Heinrichsdorff, M. H. Mao, and D. Bimberg: Ultrafast gain dynamics in InAs-InGaAs quantum-dot amplifiers, IEEE Photon. Technol. Lett. 12 (2000).

[BOR01a] P. Borri, W. Langbein, S. Schneider, U. Woggon, R. L. Sellin, D. Ouyang, and D. Bimberg: Ultralong dephasing time in InGaAs quantum dots, Phys. Rev. Lett. 87, 157401 (2001). 
[BOR02] P. Borri, W. Langbein, S. Schneider, U. Woggon, R. L. Sellin, D. Ouyang, and D. Bimberg: Exciton relaxation and dephasing in quantum-dot amplifiers from room to cryogenic temperature, IEEE J. Sel. Top. Quantum Electron. 8, 984-991 (2002).

[BOR02a] P. Borri, W. Langbein, S. Schneider, U. Woggon, R. L. Sellin, D. Ouyang, and D. Bimberg: Rabi oscillations in the excitonic ground-state transition of In GaAs quantum dots, Phys. Rev. B 66, 081306(R) (2002).

[BRA05] T. Brandes: Coherent and collective quantum optical effects in mesoscopic systems, Phys. Rep. 408, 315-474 (2005).

[BRI10] C. Brif, R. Chakrabarti, and H. Rabitz: Control of quantum phenomena: past, present and future, New J. Phys. 12, 075008 (2010).

[CAL13] G. Callsen, A. Carmele, G. Hönig, C. Kindel, J. Brunnmeier, M. Wagner, E. Stock, J. S. Reparaz, A. Schliwa, S. Reitzenstein, A. Knorr, A. Hoffmann, S. Kako, and Y. Arakawa: Steering photon statistics in single quantum dots: From one- to two-photon emission, Phys. Rev. B 87, 245314 (2013).

[CAM96] F. X. Camescasse, A. Alexandrou, D. Hulin, L. Bányai, D. B. Tran Thoai, and H. Haug: Ultrafast electron redistribution through coulomb scattering in undoped gaas: Experiment and theory, Phys. Rev. Lett. 77, 5429-5432 (1996).

[CAP14] A. Capua, O. Karni, G. Eisenstein, and J. P. Reithmaier: Rabi oscillations in a room-temperature quantum dash semiconductor optical amplifier, Phys. Rev. B 90, 045305 (2014).

[CAT07] M. A. Cataluna, E. A. Viktorov, P. Mandel, W. Sibbett, D. A. Livshits, J. Weimert, A. R. Kovsh, and E. U. Rafailov: Temperature dependence of pulse duration in a mode-locked quantum-dot laser, Appl. Phys. Lett. 90, 101102 (2007).

[CHO84] Y. Cho and M. Umeda: Chaos in laser oscillations with delayed feedback; numerical analysis and observation using semiconductor laser, J. Opt.Soc.Am. B 1, 497-498 (1984).

[CHO99] W. W. Chow and S. W. Koch: Semiconductor-Laser Fundamentals (Springer, Berlin, 1999).

[CHO03] W. W. Chow, H. C. Schneider, and M. C. Phillips: Theory of quantum-coherence phenomena in semiconductor quantum dots, Phys. Rev. A 68, 053802 (2003).

[CHO05] W. W. Chow and S. W. Koch: Theory of semiconductor quantum-dot laser dynamics, IEEE J. Quantum Electron. 41, 495-505 (2005).

[CHO10] H. Choi, V. M. Gkortsas, L. Diehl, D. Bour, S. Corzine, J. Zhu, G. Höfler, F. Capasso, F. X. Kärtner, and T. B. Norris: Ultrafast rabi flopping and coherent pulse propagation in a quantum cascade laser, Nature Photonics 4, 706-710 (2010). 
[CHO11b] W. W. Chow, M. Lorke, and F. Jahnke: Will quantum dots replace quantum wells as the active medium of choice in future semiconductor lasers?, IEEE J. Sel. Top. Quantum Electron. 17, 1349-1355 (2011).

[CHO13a] W. W. Chow and F. Jahnke: On the physics of semiconductor quantum dots for applications in lasers and quantum optics, Prog. Quantum Electron. 37, 109-184 (2013).

[CHU95] S. L. Chuang: Physics of optoelectronic devices (Wiley, New York, 1995).

[COL12a] L. A. Coldren, S. W. Corzine, and M. Mashanovitch: Diode Lasers and Photonic Integrated Circuits, Wiley series in microwave and optical enginieering (Wiley \& Sons, 2nd edition, 2012).

[COL12] J. J. Coleman: The development of the semiconductor laser diode after the first demonstration in 1962, Semicond. Sci. Technol. 27, 090207 (2012).

[CON07] D. Y. Cong, A. Martinez, K. Merghem, G. Moreau, A. Lemaitre, J. G. Provost, O. Le Gouezigou, M. Fischer, I. Krestnikov, A. R. Kovsh, and A. Ramdane: Optimisation of $\alpha$-factor for quantum dot InAs/GaAs fabry-perot lasers emitting at $1.3 \mu \mathrm{m}$, Electron. Lett. 43, 222-224 (2007).

[CON10] G. Contestabile, A. Maruta, S. Sekiguchi, K. Morito, M. Sugawara, and K. Kitayama: Cross-gain modulation in quantum-dot SOA at 1550nm, IEEE J. Quantum Electron. 46, 1696-1703 (2010).

[DAG05] B. Dagens, A. Markus, J. Chen, J. G. Provost, D. Make, O. Le Gouezigou, J. Landreau, A. Fiore, and B. Thedrez: Giant linewidth enhancement factor and purely frequency modulated emission from quantum dot laser, Electron. Lett. 41, 323-324 (2005).

[DAH87] B. Dahmani, L. Hollberg, and R. Drullinger: Frequency stabilization of semiconductor lasers by resonant optical feedback, Opt. Lett. 12, 876-878 (1987).

[DAH08b] T. Dahms, P. Hövel, and E. Schöll: Stabilizing continuous-wave output in semiconductor lasers by time-delayed feedback, Phys. Rev. E 78, 056213 (2008).

[DIN74] R. Dingle, W. Wiegmann, and C. H. Henry: Quantum states of confined carriers in very thin $A l_{x} G a_{1-x} A s-G a A s-A l_{x} G a_{1-x} A s$ heterostructures, Phys. Rev. Lett. 33, 827-830 (1974).

[MEL08] A. M. de Melo and K. Petermann: On the amplified spontaneous emission noise modeling of semiconductor optical amplifiers, Opt. Commun. 281, 4598-4605 (2008).

[DOE91] E. J. Doedel, H. B. Keller, and J. P. Kervenez: Numerical analysis and control of bifurcation problems. (I) Bifurcation in finite dimensions, Int. J. Bifurcation Chaos 1, 493-520 (1991). 
[DOE09] E. J. Doedel and B. E. Oldeman: Auto-07P: Continuation and bifurcation software for ordinary differential equations. Concordia University, Montreal, Canada (2009).

[DOM07] S. Dommers, V. V. Temnov, U. Woggon, J. Gomis, J. Martinez-Pastor, M. Lämmlin, and D. Bimberg: Complete ground state gain recovery after ultrashort double pulses in quantum dot based semiconductor optical amplifier, Appl. Phys. Lett. 90, 033508 (2007).

[DUP12] F. Duport, B. Schneider, A. Smerieri, M. Haelterman, and S. Massar: All-optical reservoir computing, Opt. Express 20, 22783-22795 (2012).

[ERI71] L. E. Erickson and A. Szabo: Spectral narrowing of dye laser output by injection of monochromatic radiation into the laser cavity, Appl. Phys. Lett. 18, 433 (1971).

[ERN07a] T. Erneux, E. A. Viktorov, and P. Mandel: Time scales and relaxation dynamics in quantum-dot lasers, Phys. Rev. A 76, 023819 (2007).

[ERN09] T. Erneux: Applied delay differential equations (Springer, 2009).

[ERN09a] T. Erneux, E. A. Viktorov, P. Mandel, T. Piwonski, G. Huyet, and J. Houlihan: The fast recovery dynamics of a quantum dot semiconductor optical amplifier, Appl. Phys. Lett. 94, 113501 (2009).

[ERN10b] T. Erneux and P. Glorieux: Laser Dynamics (Cambridge University Press, UK, 2010).

[ERN10a] T. Erneux, E. A. Viktorov, B. Kelleher, D. Goulding, S. P. Hegarty, and G. Huyet: Optically injected quantum-dot lasers, Opt. Lett. 35, 070937 (2010).

[ERZ07a] H. Erzgräber, D. Lenstra, B. Krauskopf, A. P. A. Fischer, and G. Vemuri: Feedback phase sensitivity of a semiconductor laser subject to filtered optical feedback: Experiment and theory, Phys. Rev. E 76, 026212 (2007).

[SAY94] K. El Sayed, L. Bányai, and H. Haug: Coulomb quantum kinetics and optical dephasing on the femtosecond time scale, Phys. Rev. B 50, 1541-1550 (1994).

[FAn95] W. C. W. Fang, C. G. Bethea, Y. K. Chen, and S. L. Chuang: Longitudinal spatial inhomogeneities in high-power semiconductor lasers, IEEE J. Sel. Top. Quantum Electron. 1, 117-128 (1995).

[FAN98] X. Fan, T. Takagahara, J. E. Cunningham, and H. Wang: Pure dephasing induced by exciton-phonon interactions in narrow GaAs quantum wells, Solid State Commun. 108, 857-861 (1998).

[FEH02] J. N. Fehr, M. A. Dupertuis, T. P. Hessler, L. Kappei, D. Marti, F. Salleras, M. S. Nomura, B. Deveaud, J.-Y. Emery, and B. Dagens: Hot phonons and Auger related carrier heating in semiconductor optical amplifiers, IEEE J. Quantum Electron. 38, 674 (2002). 
[FEL01] J. Feldmann, S. T. Cundiff, M. Arzberger, G. Böhm, and G. Abstreiter: Carrier capture into InAs/GaAs quantum dots via multiple optical phonon emission, J. Appl. Phys. 89, 1180 (2001).

[FEL06a] T. Feldtmann, L. Schneebeli, M. Kira, and S. W. Koch: Quantum theory of light emission from a semiconductor quantum dot, Phys. Rev. B 73, 155319 (2006).

[FIO07] A. Fiore and A. Markus: Differential gain and gain compression in quantum-dot lasers, IEEE J. Quantum Electron. 43, 287-294 (2007).

[FIO11] G. Fiol, M. Kleinert, D. Arsenijević, and D. Bimberg: $1.3 \mu \mathrm{m}$ range $40 \mathrm{GHz}$ quantum-dot mode-locked laser under external continuous wave light injection or optical feedback, Semicond. Sci. Technol. 26, 014006 (2011).

[FLE81] M. W. Fleming and A. Mooradian: Fundamental line broadening of single-mode GaAlAs diode lasers, Appl. Phys. Lett. 38, 511-513 (1981).

[FLU07] V. Flunkert and E. Schöll: Suppressing noise-induced intensity pulsations in semiconductor lasers by means of time-delayed feedback, Phys. Rev. E 76, 066202 (2007).

[FLU09] V. Flunkert, O. D'Huys, J. Danckaert, I. Fischer, and E. Schöll: Bubbling in delay-coupled lasers, Phys. Rev. E 79, 065201 (R) (2009).

[FOR07] T. Fordell and A. M. Lindberg: Experiments on the linewidth-enhancement factor of a vertical-cavity surface-emitting laser, IEEE J. Quantum Electron. 43, 6-15 (2007).

[FRA13] F. Franchello, L. D. de Souza, E. Laureto, A. A. Quivy, and I. F. L. Dias: Influence of bimodal distribution and excited state emission on photoluminescence spectra of InAs self-assembled quantum dots, J. Luminescence 137, 22-27 (2013).

[FRY93] E. S. Fry, X. Li, D. Nikonov, G. G. Padmabandu, M. O. Scully, A. V. Smith, F. K. Tittel, C. Wang, S. R. Wilkinson, and S. Y. Zhu: Atomic coherence effects within the sodium $D_{1}$ line: Lasing without inversion via population trapping, Phys. Rev. Lett. 70, 3235-3238 (1993).

[GAM96] D. Gammon, E. S. Snow, B. V. Shanabrook, D. S. Katzer, and D. Park: Homogeneous linewidths in the optical spectrum of a single gallium arsenide quantum dot, Science 273, 87-90 (1996).

[GAR85] C. W. Gardiner: Handbook of Stochastic Methods (Springer, Berlin, Heidelberg, New York, 1985).

[GAR06] P. Gartner, J. Seebeck, and F. Jahnke: Relaxation properties of the quantum kinetics of carrier-LO-phonon interaction in quantum wells and quantum dots, Phys. Rev. B 73, 115307 (2006). 
[GAV97] A. Gavrielides, V. Kovanis, P. M. Varangis, T. Erneux, and G. Lythe: Coexisting periodic attractors in injection-locked diode lasers, Quantum Semiclass. Opt. 9, 785 (1997).

[GOM08] J. Gomis-Bresco, S. Dommers, V. V. Temnov, U. Woggon, M. Lämmlin, D. Bimberg, E. Malić, M. Richter, E. Schöll, and A. Knorr: Impact of Coulomb scattering on the ultrafast gain recovery in In GaAs quantum dots, Phys. Rev. Lett. 101, 256803 (2008).

[GOM09] J. Gomis-Bresco, S. Dommers, V. V. Temnov, U. Woggon, J. Martinez-Pastor, M. Lämmlin, and D. Bimberg: InGaAs quantum dots coupled to a reservoir of nonequilibrium free carriers, IEEE J. Quantum Electron. 45, 1121-1128 (2009).

[GER08] S. Gerhard, C. Schilling, F. Gerschütz, M. Fischer, J. Koeth, I. Krestnikov, A. R. Kovsh, M. Kamp, S. Höfling, and A. Forchel: Frequency-dependent linewidth enhancement factor of quantum-dot lasers, IEEE Photonics Technol. Lett. 20, 1736-1738 (2008).

[GIE07] C. Gies, J. Wiersig, M. Lorke, and F. Jahnke: Semiconductor model for quantumdot-based microcavity lasers, Phys. Rev. A 75, 013803 (2007).

[GIE11] C. Gies, M. Florian, P. Gartner, and F. Jahnke: The single quantum dot-laser: lasing and strong coupling in the high-excitation regime, Opt. Express 19, 14370 (2011).

[GIO07] M. Gioannini and I. Montrosset: Numerical analysis of the frequency chirp in quantum-dot semiconductor lasers, IEEE J. Quantum Electron. 43, 941-949 (2007).

[GIO11] M. Gioannini and M. Rossetti: Time-domain traveling wave model of quantum dot DFB lasers, IEEE J. Sel. Top. Quantum Electron. 17, 1318-1326 (2011).

[GIO12] M. Gioannini: Ground-state quenching in two-state lasing quantum dot lasers, J. Appl. Phys. 111, 043108 (2012).

[GLO12] B. Globisch, C. Otto, E. Schöll, and K. Lüdge: Influence of carrier lifetimes on the dynamical behavior of quantum-dot lasers subject to optical feedback, Phys. Rev. E 86, 046201 (2012).

[GUE13] I. J. Guerrero Moreno, T. V. Torchynska, and J. L. Casas Espinola: Photoluminescence variation in InAs quantum dots embedded in InGaAs/AlGaAs quantum wells at thermal annealing, Physica E 51, 37-41 (2013).

[GOE98a] J. P. Goedgebuer, L. Larger, and H. Porte: Optical cryptosystem based on synchronization of hyperchaos generated by a delayed feedback tunable laser diode, Phys. Rev. Lett. 80, 2249-2252 (1998).

[GOL14] E. Goldmann, M. Lorke, T. Frauenheim, and F. Jahnke: Negative differential gain in quantum dot systems: Interplay of structural properties and many-body effects, Appl. Phys. Lett. 104, 242108 (2014). 
[GOU07] D. Goulding, S. P. Hegarty, O. Rasskazov, S. Melnik, M. Hartnett, G. Greene, J. G. McInerney, D. Rachinskii, and G. Huyet: Excitability in a quantum dot semiconductor laser with optical injection, Phys. Rev. Lett. 98, 153903 (2007).

[GRI08a] F. Grillot, B. Dagens, J. G. Provost, H. Su, and L. F. Lester: Gain compression and above-threshold linewidth enhancement factor in $1.3 \mu \mathrm{m}$ InAs/GaAs quantum-dot lasers, IEEE J. Quantum Electron. 44, 946-951 (2008).

[GRI11] F. Grillot and N. Dubey: Influence of the linewidth enhancement factor on the modulation response of a nanostructure-based semiconductor laser operating under external optical feedback, Proc. SPIE 7933, 79330E (2011).

[GRO14] N. B. Grosse, N. Owschimikow, R. Aust, B. Lingnau, A. Koltchanov, M. Kolarczik, K. Lüdge, and U. Woggon: Pump-probe quantum state tomography in a semiconductor optical amplifier, Opt. Express 22, 32520-32525 (2014).

[HAK83a] H. Haken: Laser Theory (Springer, 1983).

[HAR83] C. Harder, K. Vahala, and A. Yariv: Measurement of the linewidth enhancement factor alpha of semiconductor lasers, Appl. Phys. Lett. 42, 328-330 (1983).

[HAR89] S. E. Harris: Lasers without inversion: Interference of lifetime-broadened resonances, Phys. Rev. Lett. 62, 1033-1036 (1989).

[HAR97c] S. E. Harris: Electromagnetically induced transparency, Phys. Today 50, 36-42 (1997).

[HAR12] T. Harayama, S. Sunada, K. Yoshimura, J. Muramatsu, K. Arai, A. Uchida, and P. Davis: Theory of fast nondeterministic physical random-bit generation with chaotic lasers, Phys. Rev. E 85, 046215 (2012).

[HAU84] H. Haug and S. Schmitt-Rink: Electron theory of the optical properties of laserexcited semiconductors, Prog. Quantum Electron. 9, 3 (1984).

[HAU89] H. Haug and S. W. Koch: Semiconductor laser theory with many-body effects, Phys. Rev. A 39, 1887 (1989).

[HAU96] H. Haug and A. P. Jauho: Quantum Kinetics in Transport and Optics of Semiconductors (Springer, Berlin, 1996).

[HEI01a] T. Heil, I. Fischer, W. Elsäßer, and A. Gavrielides: Dynamics of semiconductor lasers subject to delayed optical feedback: The short cavity regime, Phys. Rev. Lett. 87, 243901 (2001).

[HEI01] R. Heitz, H. Born, F. Guffarth, O. Stier, A. Schliwa, A. Hoffmann, and D. Bimberg: Existence of a phonon bottleneck for excitons in quantum dots, Phys. Rev. B 64, 241305(R) (2001).

[HEI03a] T. Heil, I. Fischer, W. Elsäßer, B. Krauskopf, K. Green, and A. Gavrielides: Delay dynamics of semiconductor lasers with short external cavities: Bifurcation scenarios and mechanisms, Phys. Rev. E 67, 066214 (2003). 
[HEN82] C. H. Henry: Theory of the linewidth of semiconductor lasers, IEEE J. Quantum Electron. 18, 259-264 (1982).

[HEN86] C. H. Henry and R. F. Kazarinov: Instability of semiconductor lasers due to optical feedback from distant reflectors, IEEE J. Quantum Electron. 22, 294301 (1986).

[HIL02] M. T. Hill, E. E. Frietman, H. de Waardt, G.-d. Khoe, and H. J. S. Dorren: All fiber-optic neural network using coupled SOA based ring lasers, IEEE Trans. Neural Networks 13, 1504-1513 (2002).

[HIR94a] H. Hirayama, K. Matsunaga, M. Asada, and Y. Suematsu: Lasing action of $\mathrm{Ga}_{0.67} \operatorname{In}_{0.33} \mathrm{As} / \mathrm{GaInAsP} / \mathrm{InP}$ tensile-strained quantum-box laser, Electron. Lett. 30, 142-143 (1994).

[HOH99] A. Hohl and A. Gavrielides: Bifurcation cascade in a semiconductor laser subject to optical feedback, Phys. Rev. Lett. 82, 1148-1151 (1999).

[HUA96] Y. Z. Huang, Z. Pan, and R. H. Wu: Analysis of the optical confinement factor in semiconductor lasers, J. Appl. Phys. 79, 3827-3830 (1996).

[HUR13] A. Hurtado, I. D. Henning, M. J. Adams, and L. F. Lester: Generation of tunable millimeter-wave and THz signals with an optically injected quantum dot distributed feedback laser, IEEE Photonics Journal 5, 5900107 (2013).

[HUY04] G. Huyet, D. O’Brien, S. P. Hegarty, J. G. McInerney, A. V. Uskov, D. Bimberg, C. Ribbat, V. M. Ustinov, A. E. Zhukov, S. S. Mikhrin, A. R. Kovsh, J. K. White, K. Hinzer, and A. J. SpringThorpe: Quantum dot semiconductor lasers with optical feedback, phys. stat. sol. (b) 201, 345-352 (2004).

[ICS69] A. Icsevgi and W. E. Lamb: Propagation of light pulses in a laser amplifier, Phys. Rev. 185, 517-545 (1969).

[INO92] T. Inoshita and H. Sakaki: Electron relaxation in a quantum dot: Significance of multiphonon processes, Phys. Rev. B 46, 7260-7263 (1992).

[ISH04] M. Ishida, N. Hatori, T. Akiyama, K. Otsubo, Y. Nakata, H. Ebe, M. Sugawara, and Y. Arakawa: Photon lifetime dependence of modulation efficiency and $K$ factor im $1.3 \mu \mathrm{m}$ self-assembled InAs/GaAs quantum-dot lasers: Impact of capture time and maximum modal gain on modulation bandwidth, Appl. Phys. Lett. 85, 4145 (2004).

[ISH07] M. Ishida, M. Sugawara, T. Yamamoto, N. Hatori, H. Ebe, Y. Nakata, and Y. Arakawa: Theoretical study on high-speed modulation of Fabry-Pérot and distributed-feedback quantum-dot lasers: K-factor-limited bandwidth and 10 Gbit/s eye diagrams, J. Appl. Phys. 101, 013108 (2007).

[ISL87] M. N. Islam, R. L. Hillman, D. A. B. Miller, D. S. Chemla, A. C. Gossard, and J. H. English: Electroabsorption in GaAs/AlGaAs coupled quantum well waveguides, Appl. Phys. Lett. 50 (1987). 
[JAV12] J. Javaloyes and S. Balle: Multimode dynamics in bidirectional laser cavities by folding space into time delay, Opt. Express 20, 8496-8502 (2012).

[JIA12] Z. J. Jiao, Z. G. Lu, J. R. Liu, P. J. Poole, P. Barrios, D. Poitras, G. Pakulski, J. Caballero, and X. P. Zhang: Linewidth enhancement factor of InAs/InP quantum dot lasers around 1.5 $\mu$ m, Opt. Commun. 285, 4372-4375 (2012).

[JIN04] P. Jin, C. M. Li, Z. Y. Zhang, F. Q. Liu, Y. H. Chen, X. L. Ye, B. Xu, and Z. G. Wang: Quantum-confined stark effect and built-in dipole moment in selfassembled InAs/GaAs quantum dots, Appl. Phys. Lett. 85, 2791 (2004).

[JIN06] X. Jin and S. L. Chuang: Bandwidth enhancement of Fabry-Perot quantum-well lasers by injection-locking, Solid-State Electron. 50, 1141-1149 (2006).

[KAM66] I. P. Kaminow and E. H. Turner: Electrooptic light modulators, Appl. Opt. 5, $1612-1628$ (1966).

[KAM02] H. Kamada, H. Gotoh, J. Temmyo, H. Ando, and T. Takagahara: Exciton Rabi oscillation in single isolated quantum dots, phys. stat. sol. (a) 190, 485-490 (2002).

[KAN96b] M. G. Kane: Nonequilibrium carrier-carrier scattering in two-dimensional carrier systems, Phys. Rev. B 54, 16345-16348 (1996).

[KAN05] D. M. Kane and K. A. Shore (Editors): Unlocking Dynamical Diversity: Optical Feedback Effects on Semiconductor Lasers (Wiley VCH, Weinheim, 2005).

[KAP14b] Y. Kaptan, A. Röhm, B. Herzog, B. Lingnau, H. Schmeckebier, D. Arsenijević, V. Mikhelashvili, O. Schops, M. Kolarczik, G. Eisenstein, D. Bimberg, U. Woggon, N. Owschimikow, and K. Lüdge: Stability of a quantum dot excited state laser during simultaneous ground state amplification, Appl. Phys. Lett. 105, 191105-1-191105-4 (2014).

[KAP14c] Y. Kaptan, H. Schmeckebier, B. Herzog, D. Arsenijević, M. Kolarczik, V. Mikhelashvili, N. Owschimikow, G. Eisenstein, D. Bimberg, and U. Woggon: Gain dynamics of quantum dot devices for dual-state operation, Appl. Phys. Lett. 104 (2014).

[KEL09] B. Kelleher, D. Goulding, S. P. Hegarty, G. Huyet, D. Y. Cong, A. Martinez, A. Lemaitre, A. Ramdane, M. Fischer, F. Gerschütz, and J. Koeth: Excitable phase slips in an injection-locked single-mode quantum-dot laser, Opt. Lett. 34, 440-442 (2009).

[KEL11a] B. Kelleher, C. Bonatto, G. Huyet, and S. P. Hegarty: Excitability in optically injected semiconductor lasers: Contrasting quantum-well- and quantumdot-based devices, Phys. Rev. E 83, 026207 (2011).

[KEL11] B. Kelleher, D. Goulding, G. Huyet, E. A. Viktorov, T. Erneux, and S. P. Hegarty: Dimensional signature on noise-induced excitable statistics in an optically injected semiconductor laser, Phys. Rev. E 84, 026208 (2011). 
[KEL12] B. Kelleher, D. Goulding, B. Baselga Pascual, S. P. Hegarty, and G. Huyet: Bounded phase phenomena in the optically injected laser, Phys. Rev. E 85, 046212 (2012).

[KIM08] J. Kim, M. Lämmlin, C. Meuer, D. Bimberg, and G. Eisenstein: Static gain saturation model of quantum-dot semiconductor optical amplifiers, IEEE J. Quantum Electron. 44, 658 (2008).

[KIM09a] J. Kim, M. Laemmlin, C. Meuer, D. Bimberg, and G. Eisenstein: Theoretical and experimental study of high-speed small-signal cross-gain modulation of quantumdot semiconductor optical amplifiers, IEEE J. Quantum Electron. 45, 240-248 (2009).

[KIM10e] K. C. Kim, I. K. Han, J. I. Lee, and T. G. Kim: Gain-dependent linewidth enhancement factor in the quantum dot structures, Nanotechnology, 134010 (2010).

[KIM14] B. Kim, N. Li, A. Locquet, and D. S. Citrin: Experimental bifurcation-cascade diagram of an external-cavity semiconductor laser, Opt. Express 22, 2348 (2014).

[KLO99] D. Klotzkin and P. Bhattacharya: Temperature dependence of dynamic and dc characteristics of quantum-well and quantum-dot lasers: A comparative study, J. Lightwave Technol. 17, 1634 (1999).

[KOC00] S. W. Koch, T. Meier, F. Jahnke, and P. Thomas: Microscopic theory of optical dephasing in semiconductors, Appl. Phys. A 71, 511-517 (2000).

[KOG72] H. Kogelnik and C. V. Shank: Coupled-wave theory of distributed feedback lasers, J. Appl. Phys. 43, 2327-2335 (1972).

[KOL13] M. Kolarczik, N. Owschimikow, J. Korn, B. Lingnau, Y. Kaptan, D. Bimberg, E. Schöll, K. Lüdge, and U. Woggon: Quantum coherence induces pulse shape modification in a semiconductor optical amplifier at room temperature, Nat. Commun. 4, 2953 (2013).

[KON04a] P. K. Kondratko, S. L. Chuang, G. Walter, T. Chung, and N. Holonyak: Observations of near-zero linewidth enhancement factor in a quantum-well coupled quantum-dot laser, Appl. Phys. Lett. 83, 4818 (2004).

[KOP11] T. Koprucki, A. Wilms, A. Knorr, and U. Bandelow: Modeling of quantum dot lasers with microscopic treatment of Coulomb effects, Opt. Quantum Electron. 42, 777-783 (2011).

[KRA97b] B. Krauskopf, W. A. van der Graaf, and D. Lenstra: Bifurcations of relaxation oscillations in an optically injected diode laser, J. Opt. Soc. Am. B 9, 797 (1997).

[KRA07] B. Krauskopf, H. M. Osinga, and J. Galán-Vioque: Numerical Continuation Methods for Dynamical Systems: Path following and boundary value problems (Springer Verlag, 2007). 
[KRO63] H. Kroemer: A proposed class of hetero-junction injection lasers, Proc. IEEE 51, 1782-1783 (1963).

[KUN02] M. Kuntz, N. N. Ledentsov, D. Bimberg, A. R. Kovsh, V. M. Ustinov, A. E. Zhukov, and Y. M. Shernyakov: Spectrotemporal response of $1.3 \mu \mathrm{m}$ quantumdot lasers, Appl. Phys. Lett. 81, 3846-3848 (2002).

[KUN08] S. B. Kuntze, A. J. Zilkie, L. Pavel, and J. S. Aitchison: Nonlinear state-space model of semiconductor optical amplifiers with gain compression for system design and analysis, J. Lightwave Technol. 26, 2274-2281 (2008).

[LAE06] M. Lämmlin: GaAs-based semiconductor optical amplifiers with quantum dots as an active medium, Ph.d. thesis, Technische Universität Berlin (2006).

[LAE06a] M. Lämmlin, G. Fiol, C. Meuer, M. Kuntz, F. Hopfer, A. R. Kovsh, N. N. Ledentsov, and D. Bimberg: Distortion-free optical amplification of 20-80 GHz modelocked laser pulses at 1.3 $\mu \mathrm{m}$ using quantum dots, Electron. Lett. 42, 697 (2006).

[LAN73] R. Lang, M. O. Scully, and W. E. Lamb, Jr.: Why is the laser line so narrow? a theory of single-quasimode laser operation, Phys. Rev. A 7, 1788-1797 (1973).

[LAN80b] R. Lang and K. Kobayashi: External optical feedback effects on semiconductor injection laser properties, IEEE J. Quantum Electron. 16, 347-355 (1980).

[LAR10] L. Larger and J. M. Dudley: Nonlinear dynamics: Optoelectronic chaos, Nature 465, 41-42 (2010).

[LAU08a] E. K. Lau, X. Zhao, H.-K. Sung, D. Parekh, C. J. Chang-Hasnain, and M. C. Wu: Strong optical injection-locked semiconductor lasers demonstrating $>100$ Ghz resonance frequencies and 80-Ghz intrinsic bandwidths, Opt. Express 16, 6609-6618 (2008).

[LAU09a] E. K. Lau, L. J. Wong, and M. C. Wu: Enhanced modulation characteristics of optical injection-locked lasers: A tutorial, IEEE J. Sel. Top. Quantum Electron. 15, 618-633 (2009).

[LAX67] M. Lax: Classical noise. V. noise in self-sustained oscillators, Phys. Rev. 160, 290 (1967).

[LEI08] W. Lei, M. Offer, A. Lorke, C. Notthoff, C. Meier, O. Wibbelhoff, and A. D. Wieck: Probing the band structure of InAs/GaAs quantum dots by capacitancevoltage and photoluminescence spectroscopy, Appl. Phys. Lett. 92, 193111 (2008).

[LEL07] F. Lelarge, B. Dagens, J. Renaudier, R. Brenot, A. Accard, F. van Dijk, D. Make, O. L. Gouezigou, J. G. Provost, F. Poingt, J. Landreau, O. Drisse, E. Derouin, B. Rousseau, F. Pommereau, and G. H. Duan: Recent advances on InAs/InP quantum dash based semiconductor lasers and optical amplifiers operating at $1.55 \mu$ m, IEEE J. Sel. Top. Quantum Electron. 13, 111 (2007). 
[LEN91] D. Lenstra: Statistical-theory of the multistable external-feedback laser, Opt. Commun. 81, 209-214 (1991).

[LEN14] D. Lenstra and M. Yousefi: Rate-equation model for multi-mode semiconductor lasers with spatial hole burning, Opt. Express 22, 8143-8149 (2014).

[LEV95] A. M. Levine, G. H. M. van Tartwijk, D. Lenstra, and T. Erneux: Diode lasers with optical feedback: Stability of the maximum gain mode, Phys. Rev. A 52, R3436 (1995), (4 pages).

[LI13b] X. Li, T. Wang, C. Dong, J. Tang, B. Liu, and Y. He: Modified Drude model for free-carrier absorption due to bound-to-continuum transition in quantum-dot semiconductor optical amplifier, J. Appl. Phys. 114, 154506 (2013).

[LIN10e] C. Y. Lin, F. Grillot, N. A. Naderi, Y. Li, and L. F. Lester: rf linewidth reduction in a quantum dot passively mode-locked laser subject to external optical feedback, Appl. Phys. Lett. 96, 051118 (2010).

[LIN10] B. Lingnau, K. Lüdge, E. Schöll, and W. W. Chow: Many-body and nonequilibrium effects on relaxation oscillations in a quantum-dot microcavity laser, Appl. Phys. Lett. 97, 111102 (2010).

[LIN12b] B. Lingnau, K. Lüdge, W. W. Chow, and E. Schöll: Failure of the $\alpha$-factor in describing dynamical instabilities and chaos in quantum-dot lasers, Phys. Rev. E 86, 065201(R) (2012).

[LIN12] B. Lingnau, K. Lüdge, W. W. Chow, and E. Schöll: Influencing modulation properties of quantum-dot semiconductor lasers by electron lifetime engineering, Appl. Phys. Lett. 101, 131107 (2012).

[LIN12a] B. Lingnau, K. Lüdge, W. W. Chow, and E. Schöll: Many-body effects and selfcontained phase dynamics in an optically injected quantum-dot laser, in Semiconductor Lasers and Laser Dynamics V, Brussels, edited by K. Panajotov, M. Sciamanna, A. A. Valle, and R. Michalzik (SPIE, 2012), vol. 8432 of Proceedings of SPIE, pp. 84321J-1.

[LIN12t] B. Lingnau, K. Lüdge, W. W. Chow, and E. Schöll: Many-body effects and self-contained phase dynamics in an optically injected quantum-dot laser, SPIE Proc. 8432 (2012).

[LIN13] B. Lingnau, W. W. Chow, E. Schöll, and K. Lüdge: Feedback and injection locking instabilities in quantum-dot lasers: a microscopically based bifurcation analysis, New J. Phys. 15, 093031 (2013).

[LIN14] B. Lingnau, W. W. Chow, and K. Lüdge: Amplitude-phase coupling and chirp in quantum-dot lasers: influence of charge carrier scattering dynamics, Opt. Express 22, 4867-4879 (2014).

[LIN14p] B. Lingnau and K. Lüdge: Quantenpunktlaser - Laserlicht auf den Punkt gebracht, Physik unserer Zeit 45, 140-146 (2014). 
[LIN14a] B. Lingnau, E. Schöll, and K. Lüdge: Ground and Excited-State Performance of a Quantum-Dot Semiconductor Amplifier, in Proceedings of the 14th international conference on Numerical Simulation of Optoelectronic Devices, edited by (IEEE Photonics Society, 2014), pp. 121-122.

[LIU02a] Y. Liu, H. K. Liu, and Y. Braiman: Injection locking of individual broad-area lasers in an integrated high-power diode array, Appl. Phys. Lett. 81, 978 (2002).

[LIU05a] Y. Liu, W.-C. Ng, K. D. Choquette, and K. Hess: Numerical investigation of self-heating effects of oxide-confined vertical-cavity surface-emitting lasers, IEEE J. Quantum Electron. 41, 15-25 (2005).

[LOR06b] M. Lorke, W. W. Chow, T. R. Nielsen, J. Seebeck, P. Gartner, and F. Jahnke: Anomaly in the excitation dependence of the optical gain of semiconductor quantum dots, Phys. Rev. B 74, 035334 (2006).

[LOR06] M. Lorke, T. R. Nielsen, J. Seebeck, P. Gartner, and F. Jahnke: Influence of carrier-carrier and carrier-phonon correlations on optical absorption and gain in quantum-dot systems, Phys. Rev. B 73, 085324 (2006).

[LOR06a] M. Lorke, J. Seebeck, T. R. Nielsen, P. Gartner, and F. Jahnke: Excitation dependence of the homogeneous linewidths in quantum dots, phys. stat. sol. (c) 3, 2393-2396 (2006).

[LOU00] R. Loudon: The Quantum Theory of Light (Oxford Science Publications, 2000).

[LUE09] K. Lüdge and E. Schöll: Quantum-dot lasers - desynchronized nonlinear dynamics of electrons and holes, IEEE J. Quantum Electron. 45, 1396-1403 (2009).

[LUE10a] K. Lüdge, R. Aust, G. Fiol, M. Stubenrauch, D. Arsenijević, D. Bimberg, and E. Schöll: Large signal response of semiconductor quantum-dot lasers, IEEE J. Quantum Electron. 46, 1755 -1762 (2010).

[LUE10] K. Lüdge and E. Schöll: Nonlinear dynamics of doped semiconductor quantum dot lasers, Eur. Phys. J. D 58, 167-174 (2010).

[LUE11] K. Lüdge, E. Schöll, E. A. Viktorov, and T. Erneux: Analytic approach to modulation properties of quantum dot lasers, J. Appl. Phys. 109, 103112 (2011).

[LUE11a] K. Lüdge: Modeling Quantum Dot based Laser Devices, in Nonlinear Laser Dynamics - From Quantum Dots to Cryptography, edited by K. Lüdge (WILEYVCH Weinheim, Weinheim, 2012), chapter 1, pp. 3-34.

[LUE11b] K. Lüdge: Nonlinear Laser Dynamics - From Quantum Dots to Cryptography (Wiley-VCH, Weinheim, 2012).

[MAH90] G. D. Mahan: Many-Particle Physics (Plenum, New York, 1990).

[MAI60] T. H. Maiman: Stimulated optical radiation in ruby, Nature 187, 493 (1960). 
[MAJ10] N. Majer, K. Lüdge, and E. Schöll: Cascading enables ultrafast gain recovery dynamics of quantum dot semiconductor optical amplifiers, Phys. Rev. B 82, 235301 (2010).

[MAJ11] N. Majer, S. Dommers-Völkel, J. Gomis-Bresco, U. Woggon, K. Lüdge, and E. Schöll: Impact of carrier-carrier scattering and carrier heating on pulse train dynamics of quantum dot semiconductor optical amplifiers, Appl. Phys. Lett. 99, 131102 (2011).

[MAJ11a] N. Majer, K. Lüdge, and E. Schöll: Maxwell-bloch approach to four-wave mixing in quantum dot semiconductor optical amplifiers, in 11th Internat. Conf. on Numerical Simulation of Optical Devices (NUSOD), Rome 2011, edited by J. Piprek, IEEE Proc., 153-154 (2011).

[MAL06] E. Malić, K. J. Ahn, M. J. P. Bormann, P. Hövel, E. Schöll, A. Knorr, M. Kuntz, and D. Bimberg: Theory of relaxation oscillations in semiconductor quantum dot lasers, Appl. Phys. Lett. 89, 101107 (2006).

[MAR05c] A. Martinez, K. Merghem, L. Ferlazzo, C. Dupuis, A. Ramdane, J. G. Provost, B. Dagens, O. Le Gouezigou, and O. Gauthier-Lafaye: Static and dynamic measurements of the $\alpha$-factor of five-quantum-dot-layer single-mode lasers emitting at 1.3 $\mu$ m on GaAs, Appl. Phys. Lett. 86, 211115 (2005).

[MCC69] S. L. McCall and E. L. Hahn: Self-induced transparency, Phys. Rev. 183, 457485 (1969).

[MEL06] S. Melnik, G. Huyet, and A. V. Uskov: The linewidth enhancement factor $\alpha$ of quantum dot semiconductor lasers, Opt. Express 14, 2950-2955 (2006).

[MER09] K. Merghem, R. Rosales, S. Azouigui, A. Akrout, A. Martinez, F. Lelarge, G. H. Duan, G. Aubin, and A. Ramdane: Low noise performance of passively mode locked quantum-dash-based lasers under external optical feedback, Appl. Phys. Lett. 95, 131111 (2009).

[MEU08a] C. Meuer, J. Kim, M. Lämmlin, S. Liebich, A. Capua, G. Eisenstein, A. R. Kovsh, S. S. Mikhrin, I. L. Krestnikov, and D. Bimberg: Static gain saturation in quantum dot semiconductor optical amplifiers, Opt. Express 16, 8269 (2008).

[MEU10] C. Meuer, H. Schmeckebier, G. Fiol, D. Arsenijević, J. Kim, G. Eisenstein, and D. Bimberg: Cross-gain modulation and four-wave mixing for wavelength conversion in undoped and p-doped 1.3- $\mu m$ quantum dot semiconductor optical amplifiers, IEEE Photonics Journal 2, 141-151 (2010).

[MEU11] C. Meuer, C. Schmidt-Langhorst, R. Bonk, H. Schmeckebier, D. Arsenijević, G. Fiol, A. Galperin, J. Leuthold, C. Schubert, and D. Bimberg: $80 \mathrm{~Gb} / \mathrm{s}$ wavelength conversion using a quantum-dot semiconductor optical amplifier and optical filtering, Opt. Express 19, 5134-5142 (2011). 
[MIK05] S. S. Mikhrin, A. R. Kovsh, I. L. Krestnikov, A. V. Kozhukhov, D. A. Livshits, N. N. Ledentsov, Y. M. Shernyakov, I. I. Novikov, M. V. Maximov, V. M. Ustinov, and Z. I. Alferov: High power temperature-insensitive 1.3 $\mu \mathrm{m}$ InAs/InGaAs/GaAs quantum dot lasers, Semicond. Sci. Technol., 340-342 (2005).

[MIN97b] J. Minch, S. L. Chuang, C. S. Chang, W. Fang, Y. K. Chen, and T. Tanbun-Ek: Theory and experiment on the amplified spontaneous emission from distributedfeedback lasers, IEEE J. Quantum Electron. 33, 815-823 (1997).

[MOR92] J. Mørk, B. Tromborg, and J. Mark: Chaos in semiconductor lasers with optical feedback-Theory and experiment, IEEE J. Quantum Electron. 28, 93-108 (1992).

[MOS00] E. C. Mos, J. J. L. Hoppenbrouwers, M. T. Hill, M. W. Blum, J. J. H. B. Schleipen, and H. de Waardt: Optical neuron by use of a laser diode with injection seeding and external optical feedback, IEEE Trans. Neural Networks 11, 988-996 (2000).

[MUR05b] A. Murakami and K. A. Shore: Analogy between optically-driven injectionlocked laser diodes and driven damped linear oscillators, Phys. Rev. A 73, 043804-043804-9 (2005).

[NAD09] N. A. Naderi, M. Pochet, F. Grillot, N. B. Terry, V. Kovanis, and L. F. Lester: Modeling the injection-locked behavior of a quantum dash semiconductor laser, IEEE J. Sel. Top. Quantum Electron. 15, 563-571 (2009).

[NAM99] Y. Nambu, A. Tomita, H. Saito, and K. Nishi: Effects of spectral broadening and cross relaxation on the gain saturation characteristics of quantum dot laser amplifiers, Jpn. J. Appl. Phys. 38, 5087 (1999).

[NEW99a] T. C. Newell, D. J. Bossert, A. Stintz, B. Fuchs, K. J. Malloy, and L. F. Lester: Gain and linewidth enhancement factor in InAs quantum-dot laser diodes, IEEE Photonics Technol. Lett. 11, 1527-1529 (1999).

[NGU12a] R. M. Nguimdo, G. Verschaffelt, J. Danckaert, X. J. M. Leijtens, J. Bolk, and G. Van der Sande: Fast random bits generation based on a single chaotic semiconductor ring laser, Opt. Express 20, 28603-28613 (2012).

[NGU14] R. M. Nguimdo, G. Verschaffelt, J. Danckaert, and G. Van der Sande: Fast photonic information processing using semiconductor lasers with delayed optical feedback: Role of phase dynamics, Opt. Express 22, 8672-8686 (2014).

[NIE04] T. R. Nielsen, P. Gartner, and F. Jahnke: Many-body theory of carrier capture and relaxation in semiconductor quantum-dot lasers, Phys. Rev. B 69, 235314 (2004).

[NIE05] T. R. Nielsen, P. Gartner, M. Lorke, J. Seebeck, and F. Jahnke: Coulomb scattering in nitride-based self-assembled quantum dot systems, Phys. Rev. B 72, 235311 (2005). 
[NIL05] H. H. Nilsson, J. Z. Zhang, and I. Galbraith: Homogeneous broadening in quantum dots due to Auger scattering with wetting layer carriers, Phys. Rev. B 72, 205331 (2005).

[NIN90] C. Z. Ning and H. Haken: Detuned lasers and the complex Lorenz equations: Subcritical and supercritical hopf bifurcations, Phys. Rev. A 41, 3826-3837 (1990).

[NIZ01] M. Nizette, T. Erneux, A. Gavrielides, and V. Kovanis: Averaged equations for injection locked semiconductor lasers, Physica D 161, 220 (2001).

[OBR04] D. O'Brien, S. P. Hegarty, G. Huyet, and A. V. Uskov: Sensitivity of quantumdot semiconductor lasers to optical feedback, Opt. Lett. 29, 1072-1074 (2004).

[ODR10a] I. O'Driscoll, M. Hutchings, P. M. Smowton, and P. Blood: Many-body effects in InAs/GaAs quantum dot laser structures, Appl. Phys. Lett. 97, 141102 (2010).

[OHT99] J. Ohtsubo: Feedback induced instability and chaos in semiconductor lasers and their applications, Opt. Rev. 6, 1-15 (1999).

[OHT06] J. Ohtsubo: Semiconductor Lasers: Stability, Instability and Chaos (Springer, Berlin, 2005).

[OLI11] N. Oliver, M. C. Soriano, D. W. Sukow, and I. Fischer: Dynamics of a semiconductor laser with polarization-rotated feedback and its utilization for random bit generation, Opt. Lett. 36, 4632-4634 (2011).

[OLS89] N. A. Olsson: Lightwave systems with optical amplifiers, J. Lightwave Technol. 7, 1071 (1989).

[OMA88] M. J. O'Mahony: Semiconductor laser optical amplifiers for use in future fiber systems, J. Lightwave Technol. 6, 531-544 (1988).

[OSB09a] S. Osborne, K. Buckley, A. Amann, and S. O'Brien: All-optical memory based on the injection locking bistability of a two-color laser diode, Opt. Express 17, 6293-6300 (2009).

[OSB12] S. Osborne, P. Heinricht, N. Brandonisio, A. Amann, and S. O'Brien: Wavelength switching dynamics of two-colour semiconductor lasers with optical injection and feedback, Semicond. Sci. Technol. 27, 094001 (2012).

[OTS04] K. Otsubo, N. Hatori, M. Ishida, S. Okumura, T. Akiyama, Y. Nakata, H. Ebe, M. Sugawara, and Y. Arakawa: Temperature-insensitive eye-opening under 10$\mathrm{Gb} / \mathrm{s}$ modulation of $1.3 \mu \mathrm{m}$ p-doped quantum-dot lasers without current adjustments, Jpn. J. Appl. Phys. 43, L1124 (2004).

[OTT10] C. Otto, K. Lüdge, and E. Schöll: Modeling quantum dot lasers with optical feedback: sensitivity of bifurcation scenarios, phys. stat. sol. (b) 247, 829-845 (2010). 
[OTT12] C. Otto, B. Globisch, K. Lüdge, E. Schöll, and T. Erneux: Complex dynamics of semiconductor quantum dot lasers subject to delayed optical feedback, Int. J. Bifurcation Chaos 22, 1250246 (2012).

[OTT12a] C. Otto, K. Lüdge, A. G. Vladimirov, M. Wolfrum, and E. Schöll: Delay induced dynamics and jitter reduction of passively mode-locked semiconductor laser subject to optical feedback, New J. Phys. 14, 113033 (2012).

[OTT14] C. Otto: Dynamics of Quantum Dot Lasers - Effects of Optical Feedback and External Optical Injection, Springer Theses (Springer, Heidelberg, 2014).

[OTT14b] C. Otto, L. C. Jaurigue, E. Schöll, and K. Lüdge: Optimization of timing jitter reduction by optical feedback for a passively mode-locked laser, IEEE Photonics Journal 6, 1501814 (2014).

[PAQ12] Y. Paquot, F. Duport, A. Smerieri, J. Dambre, B. Schrauwen, M. Haelterman, and S. Massar: Optoelectronic reservoir computing, Scientific Reports 2 (2012).

[PAU12] J. Pausch, C. Otto, E. Tylaite, N. Majer, E. Schöll, and K. Lüdge: Optically injected quantum dot lasers - impact of nonlinear carrier lifetimes on frequency locking dynamics, New J. Phys. 14, 053018 (2012).

[PHI01] D. F. Phillips, A. Fleischhauer, A. Mair, R. L. Walsworth, and M. D. Lukin: Storage of light in atomic vapor, Phys. Rev. Lett. 86, 783-786 (2001).

[PIE01] D. Pieroux, T. Erneux, B. Haegeman, K. Engelborghs, and D. Roose: Bridges of periodic solutions and tori in semiconductor lasers subject to delay, Phys. Rev. Lett. 87, 193901 (2001).

[PIW07] T. Piwonski, I. O’Driscoll, J. Houlihan, G. Huyet, R. J. Manning, and A. V. Uskov: Carrier capture dynamics of InAs/GaAs quantum dots, Appl. Phys. Lett. 90, 122108 (2007).

[PRE07] W. H. Press, B. P. Flannery, S. A. Teukolsky, and W. T. Vettering: Numerical Recipes (3rd ed.) (Cambridge University Press, Cambridge, 2007).

[PRI95] C. Priester and M. Lannoo: Origin of self-assembled quantum dots in highly mismatched heteroepitaxy, Phys. Rev. Lett. 75, 93 (1995).

[QAS04] O. Qasaimeh: Theory of four-wave mixing wavelength conversion in quantum dot semiconductor optical amplifiers, IEEE Photonics Technol. Lett. 16, 993995 (2004).

[QUA91] W. Quade, M. Rudan, and E. Schöll: Hydrodynamic simulation of impactionization effects in p-n junctions, IEEE Trans. CAD 10, 1287 (1991).

[QUA93a] W. Quade, E. Schöll, and M. Rudan: Impact ionization within the hydrodynamic approach to semiconductor transport, Sol. State El. 36, 1493 (1993). 
[RAD06] M. Radziunas: Numerical bifurcation analysis of the traveling wave model of multisection semiconductor lasers, Physica D 213, 98-112 (2006).

[RAD07] M. Radziunas, A. Glitzky, U. Bandelow, M. Wolfrum, U. Troppenz, J. Kreissl, and W. Rehbein: Improving the Modulation Bandwidth in Semiconductor Lasers by Passive Feedback, IEEE J. Sel. Top. Quantum Electron. 13, 136-142 (2007).

[REI09a] I. Reidler, Y. Aviad, M. Rosenbluh, and I. Kanter: Ultrahigh-speed random number generation based on a chaotic semiconductor laser, Phys. Rev. Lett. 103, 024102 (2009).

[RIB03] C. Ribbat, R. L. Sellin, I. Kaiander, F. Hopfer, N. N. Ledentsov, D. Bimberg, A. R. Kovsh, V. M. Ustinov, A. E. Zhukov, and M. V. Maximov: Complete suppression of filamentation and superior beam quality in quantum-dot lasers, Appl. Phys. Lett. 82, 952-954 (2003).

[RIT10] S. Ritter, P. Gartner, C. Gies, and F. Jahnke: Emission properties and photon statistics of a single quantum dot laser, Opt. Express 18, 9909 (2010).

[ROD05a] S. Rodt, A. Schliwa, K. Pötschke, F. Guffarth, and D. Bimberg: Correlation of structural and few-particle properties of self-organized InAs/GaAs quantum dots, Phys. Rev. B 71, 155325 (2005).

[ROE15a] A. Röhm, B. Lingnau, and K. Lüdge: Understanding ground-state quenching in quantum-dot lasers, IEEE J. Quantum Electron. 51, 2000211 (2015).

[ROM14] M. Romanelli, L. Wang, M. Brunel, and M. Vallet: Measuring the universal synchronization properties of driven oscillators across a hopf instability, Opt. Express 22, 7364 (2014).

[ROS92c] M. H. Rose, M. Lindberg, W. W. Chow, S. W. Koch, and M. Sargent: Compositecavity-mode approach to single-mode semiconductor-laser feedback instabilities, Phys. Rev. A 46, 603-611 (1992).

[ROS09a] M. Rossetti, A. Fiore, G. Sek, C. Zinoni, and L. Li: Modeling the temperature characteristics of InAs/GaAs quantum dot lasers, J. Appl. Phys. 106, 023105 (2009).

[ROS11d] M. Rossetti, P. Bardella, and I. Montrosset: Modeling passive mode-locking in quantum dot lasers: A comparison between a finite-difference traveling-wave model and a delayed differential equation approach, IEEE J. Quantum Electron. 47, 569 (2011).

[ROS11c] M. Rossetti, P. Bardella, and I. Montrosset: Time-domain travelling-wave model for quantum dot passively mode-locked lasers, IEEE J. Quantum Electron. 47, 139 (2011).

[ROZ75] N. N. Rozanov: Kinetics of a solid-state laser with an additional moving mirror, Sov. J. Quant. Electron. 4, 1191 (1975). 
[SAL92] F. Salin and J. Squier: Gain guiding in solid-state lasers, Opt. Lett. 17, 13521354 (1992).

[SCH58] A. L. Schawlow and C. H. Townes: Infrared and optical masers, Phys. Rev. 112, 1940 (1958).

[SCH86h] L. Schultheis, A. Honold, J. Kuhl, K. Köhler, and C. W. Tu: Phase coherence and line broadening of free excitons in gaas quantum wells, Superlattices and Microstructures 2, 441-443 (1986).

[SCH87] E. Schöll: Nonequilibrium Phase Transitions in Semiconductors (Springer, Berlin, 1987).

[SCH87a] E. Schöll and W. Quade: Effect of impact ionization on hot carrier energy and momentum relaxation in semiconductors, J. Phys. C 20, L 861 (1987).

[SCH88j] E. Schöll: Dynamic theory of picosecond optical pulse shaping by gain-switched semiconductor laser amplifiers, IEEE J. Quantum Electron. 24, 435-442 (1988).

[SCH88i] E. Schöll and M. Schell: Theory of ultrashort pulse generation and amplification by gain-switched semiconductor lasers, phys. stat. sol. (b) 150, 575-579 (1988).

[SCH881] N. Schunk and K. Petermann: Numerical analysis of the feedback regimes for a single-mode semiconductor laser with external feedback, IEEE J. Quantum Electron. 24, 1242-1247 (1988).

[SCH90b] M. Schell and E. Schöll: Time-dependent simulation of a semiconductor laser amplifier: Pulse compression in a ring configuration and dynamic optical bistability, IEEE J. Quantum Electron. 26, 1005-1003 (1990).

[SCH99] E. Schöll: Modelling of devices for optoelectronic applications: The quantum confined Stark effect and self-electrooptic effect devices, Turk. J. Phys. 23, 635 (1999).

[SCH01d] H. C. Schneider, W. W. Chow, and S. W. Koch: Many-body effects in the gain spectra of highly excited quantum dot lasers, Phys. Rev. B 64, 115315 (2001).

[SCH03g] S. Schneider, P. Borri, W. Langbein, U. Woggon, J. Förstner, A. Knorr, R. L. Sellin, D. Ouyang, and D. Bimberg: Self-induced transparency in InGaAs quantum-dot waveguides, Appl. Phys. Lett. 83, 3668-3670 (2003).

[SCH04e] H. C. Schneider, W. W. Chow, and S. W. Koch: Excitation-induced dephasing in semiconductor quantum dots, Phys. Rev. B 70, 235308 (2004).

[SCH05d] S. Schneider, P. Borri, W. Langbein, U. Woggon, R. L. Sellin, D. Ouyang, and D. Bimberg: Excited-state gain dynamics in InGaAs quantum-dot amplifiers, IEEE Photon. Technol. Lett. 17, 2014-2016 (2005).

[SCH06a] S. Schikora, P. Hövel, H. J. Wünsche, E. Schöll, and F. Henneberger: Alloptical noninvasive control of unstable steady states in a semiconductor laser, Phys. Rev. Lett. 97, 213902 (2006). 
[SCH07f] A. Schliwa, M. Winkelnkemper, and D. Bimberg: Impact of size, shape, and composition on piezoelectric effects and electronic properties of $\operatorname{In}(\mathrm{Ga}) \mathrm{As} / \mathrm{GaAs}$ quantum dots, Phys. Rev. B 76, 205324 (2007).

[SCH07] E. Schöll and H. G. Schuster (Editors): Handbook of Chaos Control (Wiley-VCH, Weinheim, 2008), Second completely revised and enlarged edition.

[SCH09a] E. Schöll, P. Hövel, V. Flunkert, and M. A. Dahlem: Time-delayed feedback control: from simple models to lasers and neural systems, in Complex time-delay systems: theory and applications, edited by F. M. Atay (Springer, Berlin, 2010), pp. $85-150$.

[SCH12e] H. Schmeckebier, C. Meuer, D. Arsenijević, G. Fiol, C. Schmidt-Langhorst, C. Schubert, G. Eisenstein, and D. Bimberg: Wide-range wavelength conversion of 40-Gb/s NRZ-DPSK signals using a 1.3- $\mu m$ quantum-dot semiconductor optical amplifier, IEEE Photonics Technol. Lett. 24, 1163-1165 (2012).

[SCH13k] K. Schuh, P. Gartner, and F. Jahnke: Combined influence of carrier-phonon and coulomb scattering on the quantum-dot population dynamics, Phys. Rev. B 87, 035301 (2013).

[SCH13g] F. Schulze, B. Lingnau, S. M. Hein, A. Carmele, E. Schöll, K. Lüdge, and A. Knorr: Feedback-induced steady-state light bunching above the lasing threshold, Phys. Rev. A 89, 041801(R) (2014).

[SCH15] H. Schmeckebier, B. Lingnau, S. König, K. Lüdge, C. Meuer, A. Zeghuzi, D. Arsenijević, M. Stubenrauch, R. Bonk, C. Koos, C. Schubert, T. Pfeiffer, and D. Bimberg: Ultra-broadband bidirectional dual-band quantum-dot semiconductor optical amplifier, Optical Fiber Communication Conference and Exposition Tu3I.7 (2015).

[SCU89] M. O. Scully, S. Y. Zhu, and A. Gavrielides: Degenerate quantum-beat laser: Lasing without inversion and inversion without lasing, Phys. Rev. Lett. 62, 2813-2816 (1989).

[SCU97] M. O. Scully: Quantum Optics (Cambridge University Press, 1997).

[SEE05] J. Seebeck, T. R. Nielsen, P. Gartner, and F. Jahnke: Polarons in semiconductor quantum dots and their role in the quantum kinetics of carrier relaxation, Phys. Rev. B 71, 125327 (2005).

[SEE09] J. Seebeck, M. Lorke, P. Gartner, and F. Jahnke: Carrier-carrier and carrierphonon scattering in the low-density and low-temperature regime for resonantly pumped semiconductor quantum dots, phys. stat. sol. (c) 6 (2009).

[SHA85] S. A. Shakir and W. W. Chow: Semiclassical theory of coupled lasers, Phys. Rev. A 32, 983-991 (1985).

[SHA96] J. Shah: Ultrafast Spectroscopy of Semiconductors and Semiconductor Nanostructures (Springer, Berlin, 1996). 
[SHA11b] H. Shahid, D. Childs, B. J. Stevens, and R. A. Hogg: Negative differential gain due to many body effects in self-assembled quantum dot lasers, Appl. Phys. Lett. 99, 061104 (2011).

[SHC00] O. B. Shchekin, G. Park, D. L. Huffaker, and D. G. Deppe: Discrete energy level separation and the threshold temperature dependence of quantum dot lasers, Appl. Phys. Lett. 77 (2000).

[SHO52] W. Shockley and W. T. Read: Statistics of the recombinations of holes and electrons, Phys. Rev. 87, 835-842 (1952).

[SIM94a] T. B. Simpson, J. M. Liu, A. Gavrielides, V. Kovanis, and P. M. Alsing: Perioddoubling route to chaos in a semiconductor laser subject to optical injection, Appl. Phys. Lett. 64, 3539-3541 (1994).

[SMO02] P. M. Smowton, E. J. Pearce, H. C. Schneider, W. W. Chow, and M. Hopkinson: Filamentation and linewidth enhancement factor in In GaAs quantum dot lasers, Appl. Phys. Lett. 81, 3251-3253 (2002).

[SOR13] M. C. Soriano, J. García-Ojalvo, C. R. Mirasso, and I. Fischer: Complex photonics: Dynamics and applications of delay-coupled semiconductors lasers, Rev. Mod. Phys. 85, 421-470 (2013).

[SPA84] P. Spano, S. Piazzolla, and M. Tamburrini: Theory of noise in semiconductorlasers in the presence of optical feedback, IEEE J. Quantum Electron. 20, 350357 (1984).

[STE12] A. Steinhoff, P. Gartner, M. Florian, and F. Jahnke: Treatment of carrier scattering in quantum dots beyond the boltzmann equation, Phys. Rev. B 85, 205144 (2012).

[STE13] A. Steinhoff, H. Kurtze, P. Gartner, M. Florian, D. Reuter, A. D. Wieck, M. Bayer, and F. Jahnke: Combined influence of Coulomb interaction and polarons on the carrier dynamics in InGaAs quantum dots, Phys. Rev. B 88, 205309 (2013).

[STI01b] T. H. Stievater, X. Li, D. G. Steel, D. Gammon, D. S. Katzer, D. Park, C. Piermarocchi, and L. J. Sham: Rabi oscillations of excitons in single quantum dots, Phys. Rev. Lett. 87, 133603 (2001).

[STR38] I. N. Stranski and L. Krastanow: Abhandlungen der MathematischNaturwissenschaftlichen Klasse IIb, Akademie der Wissenschaften Wien 146, 797-810 (1938).

[STR89] G. B. Stringfellow: Organometallic Vapor-Phase Epitaxy (Academic Press, New York, 1989).

[SU05a] H. Su and L. F. Lester: Dynamic properties of quantum dot distributed feedback lasers: high speed, linewidth and chirp, J. Phys. D: Appl. Phys. 38, 2112-2118 (2005). 
[SUG05] M. Sugawara, N. Hatori, M. Ishida, H. Ebe, Y. Arakawa, T. Akiyama, K. Otsubo, T. Yamamoto, and Y. Nakata: Recent progress in self-assembled quantumdot optical devices for optical telecommunication: temperature-insensitive $10 \mathrm{Gbs}$ directly modulated lasers and 40 Gbs signal-regenerative amplifiers, J. Phys. D 38, 2126-2134 (2005).

[TAN04] K. T. Tan, C. Marinelli, M. G. Thompson, A. Wonfor, M. Silver, R. L. Sellin, R. V. Penty, I. H. White, M. Kuntz, M. Lämmlin, N. N. Ledentsov, D. Bimberg, A. E. Zhukov, V. M. Ustinov, and A. R. Kovsh: High bit rate and elevated temperature data transmission using InGaAs quantum-dot lasers, IEEE Photon. Technol. Lett. 16, 1415 (2004).

[TAR95] S. Tarucha, T. Honda, and T. Saku: Reduction of quantized conductance at low temperatures observed in 2 to $10 \mu \mathrm{m}$-long quantum wires, Solid State Commun. 94, 413 (1995).

[TER08a] N. B. Terry, N. A. Naderi, M. Pochet, A. J. Moscho, L. F. Lester, and V. Kovanis: Bandwidth enhancement of injection-locked $1.3 \mu \mathrm{m}$ quantum-dot DFB laser, Electron. Lett. 44, 904-905 (2008).

[THE11] J. Thévenin, M. Romanelli, M. Vallet, M. Brunel, and T. Erneux: Resonance assisted synchronization of coupled oscillators: Frequency locking without phase locking, Phys. Rev. Lett. 107, 104101 (2011).

[TOF92] Z. Toffano, A. Destrez, C. Birocheau, and L. Hassine: New linewidth enhancement determination method in semiconductor lasers based on spectrum analysis above and below threshold, Electron. Lett. 28, 9-11 (1992).

[TON06] C. Z. Tong, S. F. Yoon, C. Y. Ngo, C. Y. Liu, and W. K. Loke: Rate Equations for 1.3- $\mu m$ Dots-Under-a-Well and Dots-in-a-Well Self-Assembled InAs-GaAs Quantum-Dot Lasers, IEEE J. Quantum Electron. 42, 1175-1183 (2006).

[TON09] C. Tong, D. Xu, and S. F. Yoon: Carrier Relaxation and Modulation Response of 1.3-mum InAs-GaAs Quantum Dot Lasers, J. Lightwave Technol. 27, 5442-5450 (2009).

[TRE85] J. R. Tredicce, F. T. Arecchi, G. L. Lippi, and G. P. Puccioni: Instabilities in lasers with an injected signal, J. Opt. Soc. Am. B 2, 173-183 (1985).

[TRO08] V. Z. Tronciu, C. R. Mirasso, and P. Colet: Chaos-based communications using semiconductor lasers subject to feedback from an integrated double cavity, J. Phys. B: At. Mol. Opt. Phys. 41, 155401 (2008).

[TRA92] D. B. Tran Thoai and H. Haug: Coulomb quantum kinetics in pulse-excited semiconductors, Z. Phys. B 91, 199-207 (1992).

[UCH12] A. Uchida: Optical Communication with Chaotic Lasers, Optical Communication with Chaotic Lasers: Applications of Nonli (Wiley, 2012). 
[UNR12] W. Unrau, D. Quandt, J. H. Schulze, T. Heindel, T. D. Germann, O. Hitzemann, A. Strittmatter, S. Reitzenstein, U. W. Pohl, and D. Bimberg: Electrically driven single photon source based on a site-controlled quantum dot with self-aligned current injection, Applied Physics Letters 101 (2012).

[URA02] J. Urayama, T. B. Norris, H. Jiang, J. Singh, and P. Bhattacharya: Temperaturedependent carrier dynamics in self-assembled In GaAs quantum dots, Appl. Phys. Lett. 80, 2162-2164 (2002).

[USK97a] A. V. Uskov, F. Adler, H. Schweizer, and M. H. Pilkuhn: Auger carrier relaxation in self-assembled quantum dots by collisions with two-dimensional carriers, J. Appl. Phys. 81, 7895 (1997).

[USK04] A. V. Uskov, E. P. O'Reilly, D. McPeake, D. Bimberg, G. Huyet, and N. N. Ledentsov: Carrier-induced refractive index in quantum dot structures due to transitions from discrete quantum dot levels to continuum states, Appl. Phys. Lett. 84, 272-274 (2004).

[USK05] A. V. Uskov, E. P. O’Reilly, M. Lämmlin, N. N. Ledentsov, and D. Bimberg: On gain saturation in quantum dot semiconductor optical amplifiers, Opt. Commun. 248, 211 (2005).

[USK11] A. V. Uskov, C. Meuer, H. Schmeckebier, and D. Bimberg: Auger capture induced carrier heating in quantum dot lasers and amplifiers, Appl. Phys. Express 4, 022202 (2011).

[VAL08] T. Vallaitis, C. Koos, R. Bonk, W. Freude, M. Laemmlin, C. Meuer, D. Bimberg, and J. Leuthold: Slow and fast dynamics of gain and phase in a quantum dot semiconductor optical amplifier, Opt. Express 16, 170-178 (2008).

[VAL13] M. Vallone: Quantum well electron scattering rates through longitudinal opticphonon dynamical screened interaction: An analytic approach, J. Appl. Phys. 114, 053704 (2013).

[DRU01] N. J. van Druten, S. S. R. Oemrawsingh, Y. Lien, C. Serrat, M. P. van Exter, and J. P. Woerdman: Observation of transverse modes in a microchip laser with combined gain and index guiding, J. Opt. Soc. Am. B 18, 1793-1804 (2001).

[POE05] M. van der Poel, E. Gehrig, O. Hess, D. Birkedal, and J. M. Hvam: Ultrafast gain dynamics in quantum-dot amplifiers: Theoretical analysis and experimental investigations, IEEE J. Quantum Electron. 41, 1115-1123 (2005).

[TAR95a] G. H. M. van Tartwijk and D. Lenstra: Semiconductor laser with optical injection and feedback, Quantum Semiclass. Opt. 7, 87-143 (1995).

[TAR98a] G. H. M. van Tartwijk and G. P. Agrawal: Laser instabilities: a modern perspective, Prog. Quantum Electronics 22, 43-122 (1998). 
[VU06] Q. T. Vu, H. Haug, and S. W. Koch: Relaxation and dephasing quantum kinetics for a quantum dot in an optically excited quantum well, Phys. Rev. B 73, 205317 (2006).

[WAL01] T. Walther, A. G. Cullis, D. J. Norris, and M. Hopkinson: Nature of the stranskikrastanow transition during epitaxy of In GaAs on GaAs, Phys. Rev. Lett. 86, 2381-2384 (2001).

[WAN97a] J. Wang and H. Schweizer: A quantitative comparison of the classical rateequation model with the carrier heating model on dynamics of the quantum-well laser: The role of carrier energy relaxation, electron-hole interaction, and auger effect, IEEE J. Quantum Electron. 33, 1350-1359 (1997).

[WAN12a] C. Wang, F. Grillot, and J. Even: Impacts of wetting layer and excited state on the modulation response of quantum-dot lasers, IEEE J. Quantum Electron. 48, 1144-1150 (2012).

[WAN14b] C. Wang, B. Lingnau, K. Lüdge, J. Even, and F. Grillot: Enhanced dynamic performance of quantum dot semiconductor lasers operating on the excited state, IEEE J. Quantum Electron. 50, 723-731 (2014).

[WEG10] M. Wegert, N. Majer, K. Lüdge, S. Dommers-Völkel, J. Gomis-Bresco, A. Knorr, U. Woggon, and E. Schöll: Nonlinear gain dynamics of quantum dot optical amplifiers, Semicond. Sci. Technol. 26, 014008 (2011).

[WEI88] C. O. Weiss, N. B. Abraham, and U. Hübner: Homoclinic and heteroclinic chaos in a single-mode laser, Phys. Rev. Lett. 61, 1587-1590 (1988).

[WEN06] Y. C. Wen, C. Y. Chen, C. H. Shen, S. Gwo, and C. K. Sun: Ultrafast carrier thermalization in InN, Appl. Phys. Lett. 89, 232114 (2006).

[WIE30] N. Wiener: Generalized harmonic analysis, Acta Mathematica 55, 117-258 (1930).

[WIE05] S. Wieczorek, B. Krauskopf, T. Simpson, and D. Lenstra: The dynamical complexity of optically injected semiconductor lasers, Phys. Rep. 416, 1-128 (2005).

[WIL12b] A. Wilms, D. Breddermann, and P. Mathe: Theory of direct capture from twoand three-dimensional reservoirs to quantum dot states, Phys. stat. sol. (c) $\mathbf{9}$, $1278-1280$ (2012).

[WIL12c] S. Wilkinson, B. Lingnau, J. Korn, E. Schöll, and K. Lüdge: Influence of noise on the signal properties of quantum-dot semiconductor optical amplifiers, IEEE J. Sel. Top. Quantum Electron. 19, 1900106 (2013).

[ZEG88] H. Zeghlache, P. Mandel, N. B. Abraham, and C. O. Weiss: Phase and amplitude dynamics in the laser Lorenz model, Phys. Rev. A 38, 3128-3131 (1988). 
[ZIE13] D. Ziemann, R. Aust, B. Lingnau, E. Schöll, and K. Lüdge: Optical injection enables coherence resonance in quantum-dot lasers, Europhys. Lett. 103, 14002p1-14002-p6 (2013).

[ZIM97a] M. G. Zimmermann, M. A. Natiello, and H. G. Solari: Shilnikov-saddle-node interaction near a codimension 2 bifurcation: Laser with injected signal, Physica D 109, 293-314 (1997).

[ZUC88] J. E. Zucker, T. L. Hendrickson, and C. A. Burrus: Electro-optic phase modulation in GaAs/AlGaAs quantum well waveguides, Appl. Phys. Lett. 52 (1988). 


\section{ACKNOWLEDGMENTS}

I am grateful to Eckehard Schöll for his support and giving me the possibility to be part of his group. Without him and his constant enthusiasm I could not have worked on the many interesting topics I had the opportunity to investigate in the last years.

I would like to thank Kathy Lüdge for the friendly working atmosphere and the constant encouragement and helpful suggestions. The many discussions in the weekly Jour Fixe - whether on or off-topic - are always interesting and enlightening, and foremost enjoyable. Thanks are also due for the thorough revision of this manuscript.

My thanks go to Weng Chow for the many insightful discussions and the fruitful collaborations. Furthermore, for being part of the examining board for this thesis.

I am thankful to all members of AG Schöll for the friendly and familiar atmosphere, the frequent social events, and the enjoyable coffee breaks. Special thanks go to Miriam Wegert for sharing the office with me and proof-reading this manuscript, Christoph Redlich and André Röhm for entertaining all nearby offices, Lina Jaurigue for coming to my office for discussions, coffee, or Skat, Judith Lehnert for many years worth of coffee-making and together with Philipp Hövel for our weekly running event, and Roland Aust for coffee-making (with Milchschaum), critical discussions of Mensa food, and fixing computers.

Furthermore I would like to thank Mirco Kolarczik, Nina Owschimikow, Yücel Kaptan, and Holger Schmeckebier for our theoretical-experimental collaborations.

Thanks also go to my family for their constant support, and getting me interested in physics in the first place. 